\title{
A mifepristone-regulated adeno-associated viral vector system for regulated neurotrophic factor expression in the central nervous system.
}

\author{
PhD Thesis \\ For the fulfillment of the requirements for the degree \\ "RerNat-O" \\ at the Georg August University Göttingen, \\ Faculty of Biology \\ submitted by \\ Andrea Maddalena \\ born in \\ Schio -VI- (Italy) \\ Göttingen 2012
}




\section{Declaration}

I hereby declare that the thesis:

"A mifepristone-regulated adeno-associated viral vector system for regulated neurotrophic factor expression in the central nervous system"

has been written independently and with no other sources and aids than quoted.

Göttingen, December 2012

Andrea Maddalena 
Supervisor:

Thesis committee:

Extended thesis committee:
Dr. Sebastian Kügler

Prof. Dr. Nils Brose

Prof. Dr. André Fischer

Ph.D. Camin Dean

Prof. Dr. Silvio Rizzoli

Prof. Dr. Tiago Outeiro 


\section{Contents}

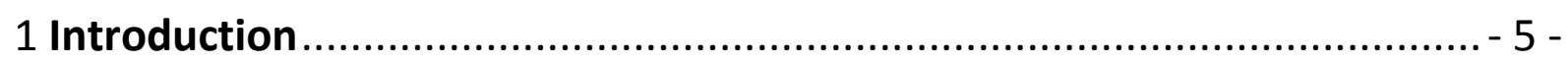

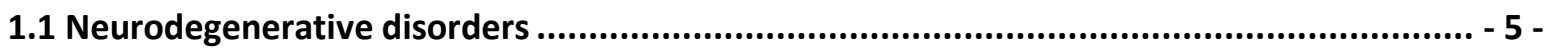

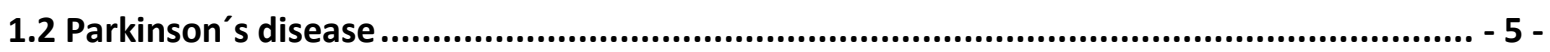

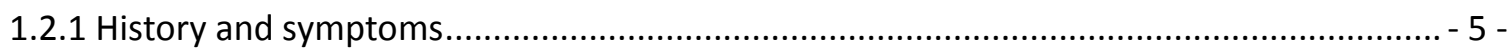

1.2.2 Causes, pathophysiology, treatment and social incidence of PD....................................... - 5 -

1.2.3 Gene therapy for Parkinson's disease treatment............................................................. 6 -

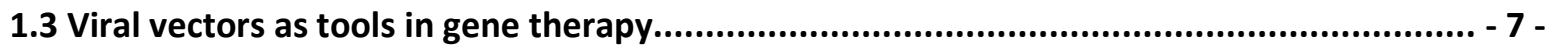

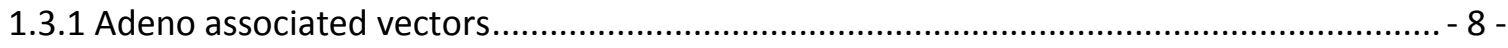

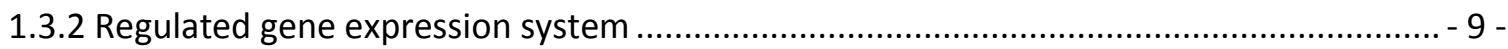

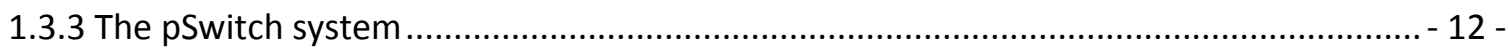

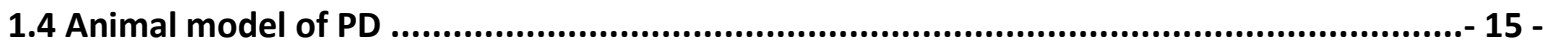

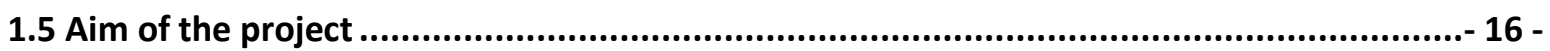

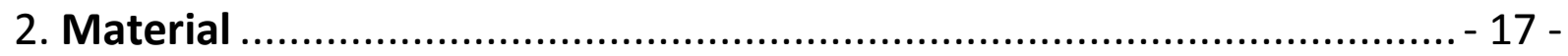

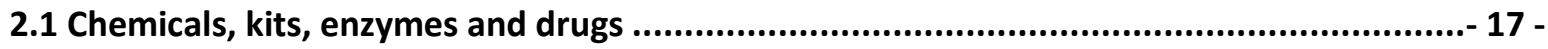

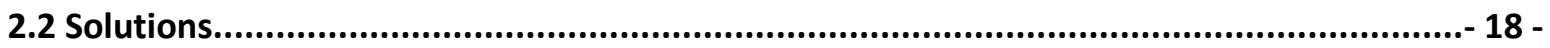

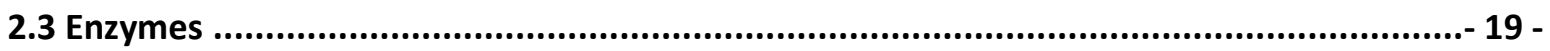

2.4 Kits

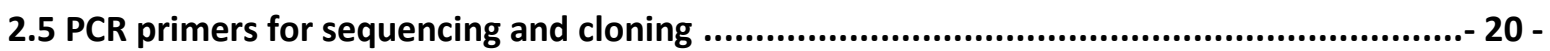

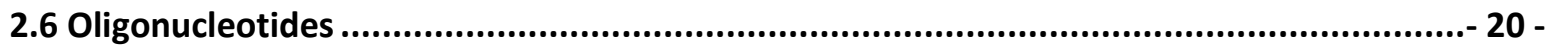

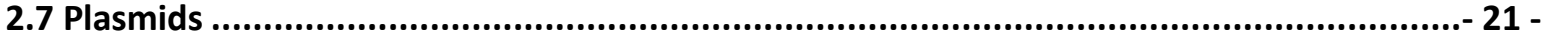

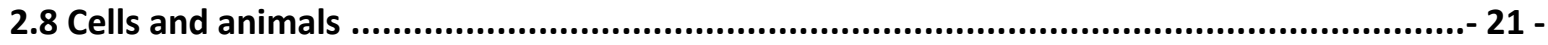

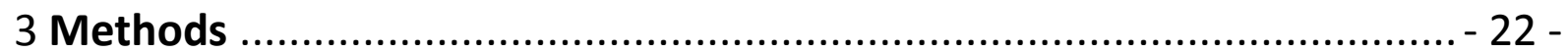

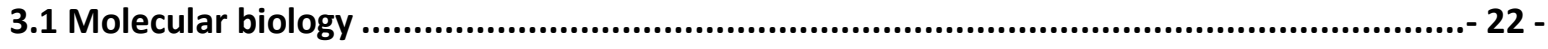

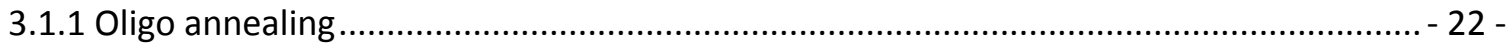

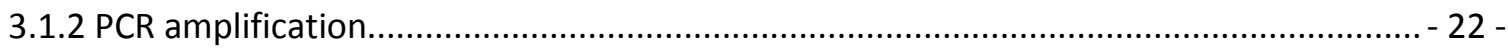

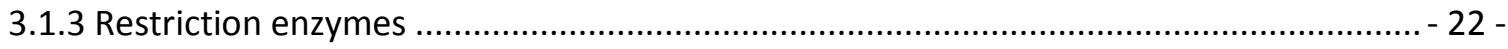

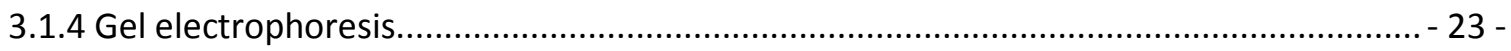

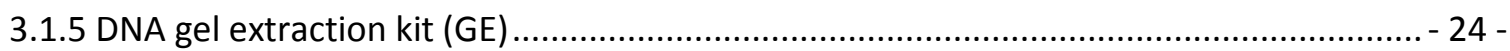

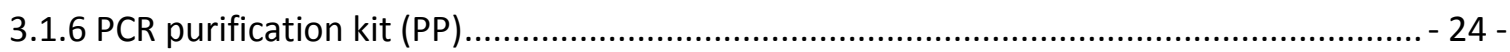

3.1.7 Remove or fill in DNA overhangs to create blunt ends .................................................... 24 -

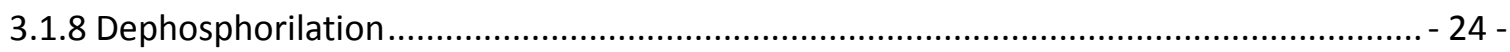

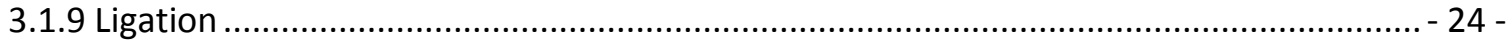


3.1.10 E.Coli transformation, seeding and expansion.......................................................... 25 -

3.1.11 Production of electro-competent E.Coli cells............................................................. - 25 -

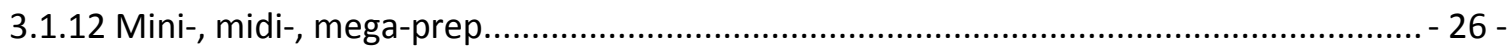

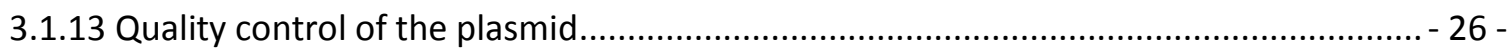

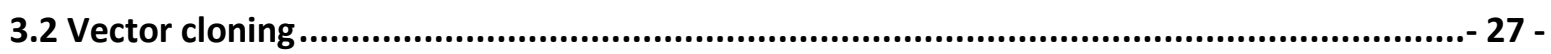

3.2.1 AAV vector genomes expressing the pSwitch protein ................................................... 28 -

3.2.2 AAV vector genome with responsive promoter ............................................................ - 28 -

3.2.3 Double cassette AAV vector genomes ………............................................................... - 29 -

3.2.4 AAV vector genomes for dopaminergic neuron generation ............................................. - 30 -

3.2.5 AAV vector genomes for control viruses ........................................................................ 31 -

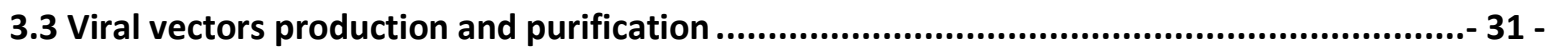

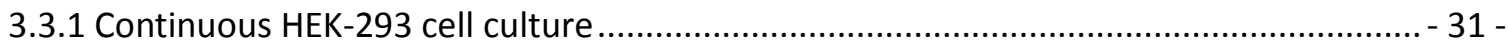

3.3.2 Calcium phosphate transfection of HEK-293 cells........................................................ 32 -

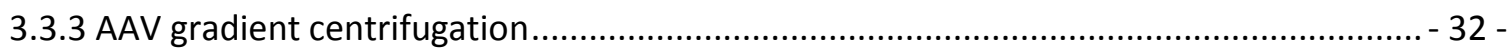

3.3.4 Fast Protein Liquid Chromatography and dialysis (FPLC) ............................................. 33 -

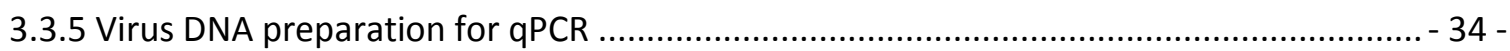

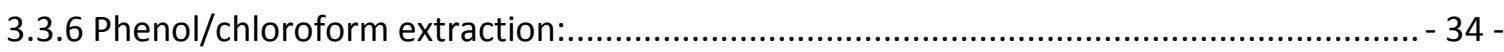

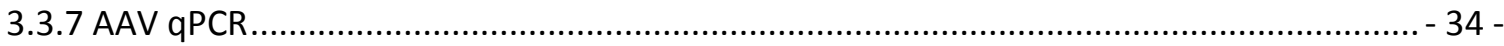

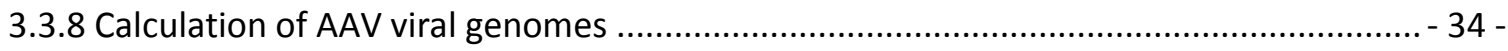

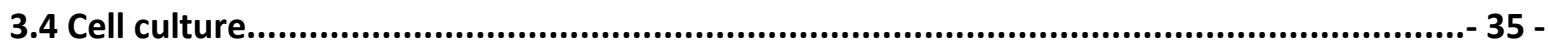

3.4.1 Coating of culture plates for primary cortical cells culture ............................................. 36 -

3.4.2 Primary cortical cells culture .......................................................................................... 36 -

3.4.3 Infection of primary cortical neurons.................................................................................... 36 -

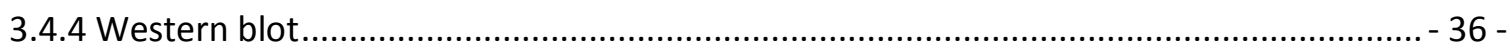

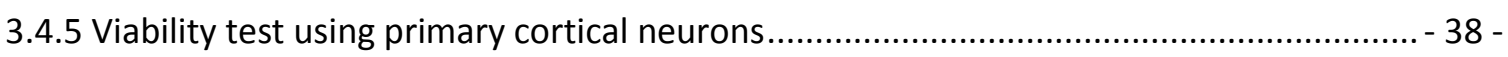

3.4.6 HPLC with cell lysates of primary cortical neurons ......................................................... 39 -

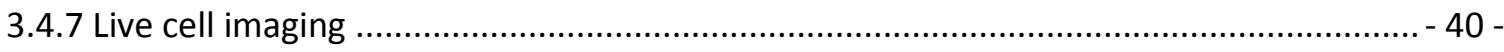

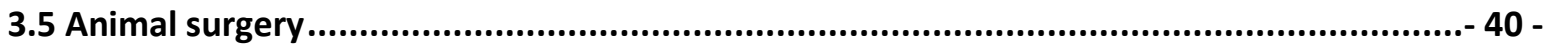

3.5.1 Cranial window implantation in mice........................................................................ 41 -

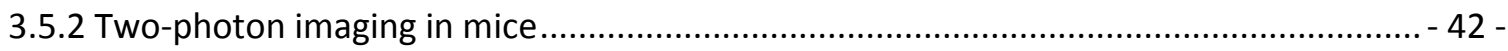

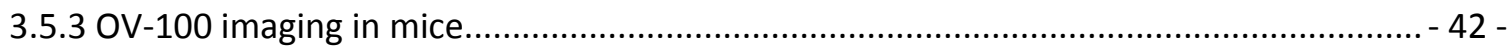

3.5.4 Transcardial perfusion and processing on mice brains ................................................... - 43 -

3.5.5 Stereotaxic injection of AAV vectors into rat striatum ..................................................... 43 -

3.5.6 Transcardial perfusion and processing on rats brains ...................................................... 44 -

3.5.7 Processing of brain tissue for catecholamines and GDNF quantification ......................... - 45 - 


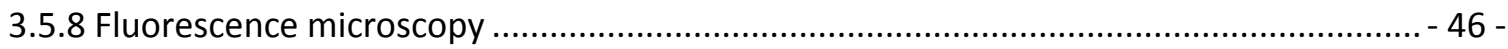

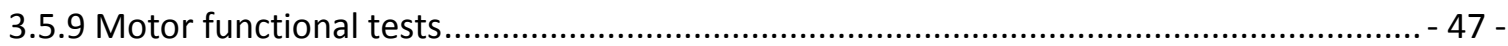

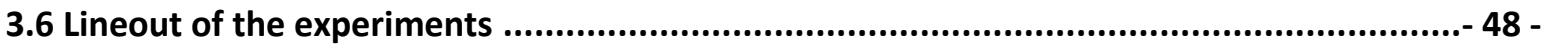

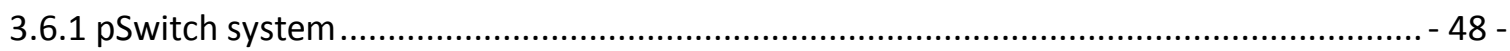

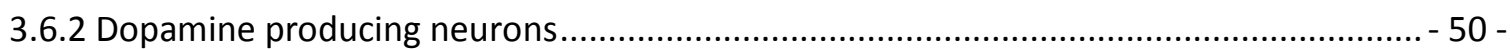

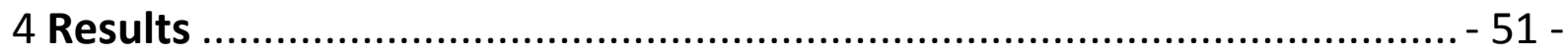

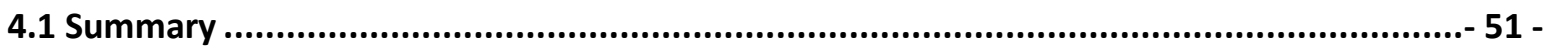

4.2 Generation of a low background regulated transgene expression system ......................... 51 -

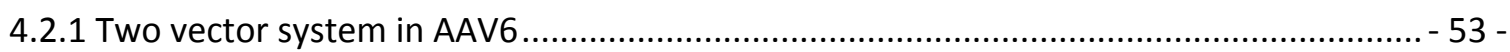

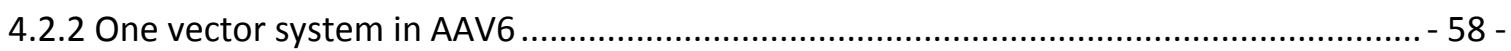

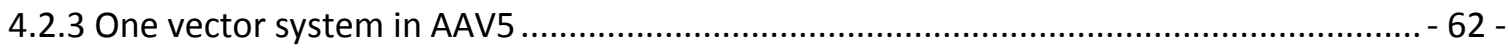

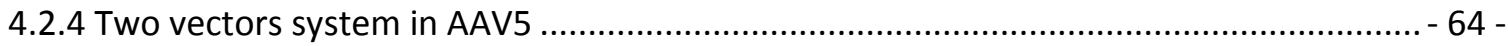

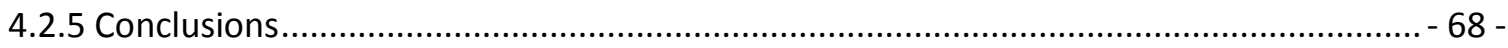

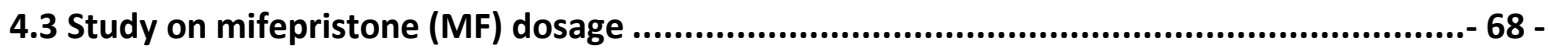

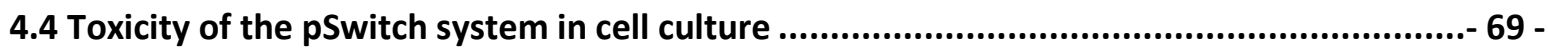

4.5 Reiterate activation of the pSwitch system in vivo ......................................................... 70 -

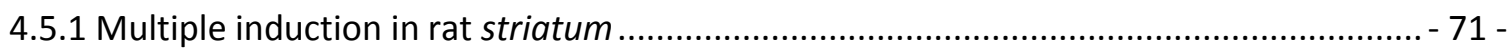

4.5.2 Reiterate imaging in mouse cortex with 2-photon microscopy ...................................... 72 -

4.5.3 Reiterate imaging in mice cortex with the OV-100 microscope....................................... 73 -

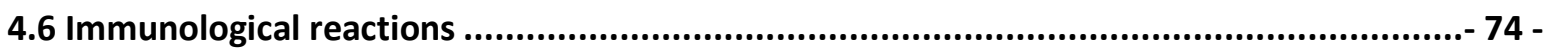

4.7 "Leakiness" of the pSwitch system in the not induced state .............................................. 75 -

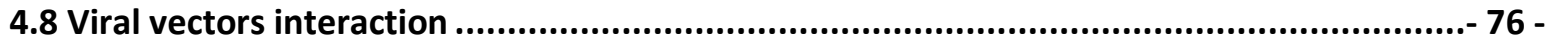

4.8.1 Interaction between different serotypes (AAV5 and AAV6) ......................................... 76 -

4.8.2 Interaction between viruses of the same serotype (AAV5) and different promoters ..... - 77 -

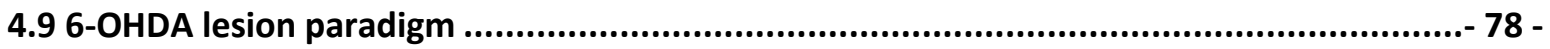

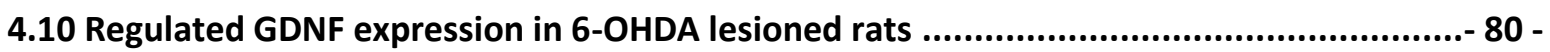

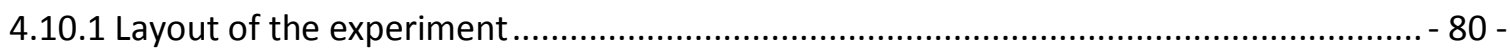

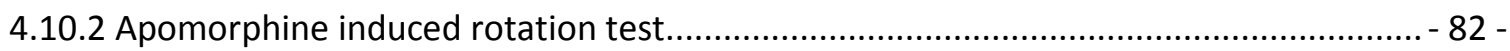

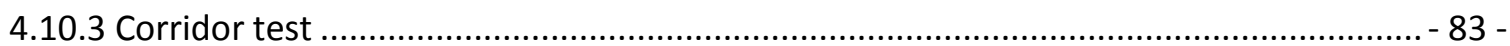

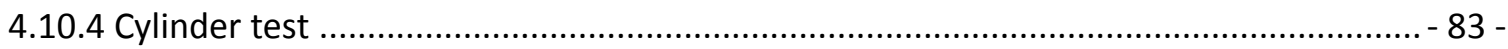

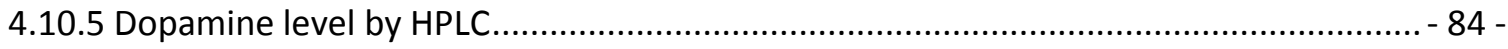

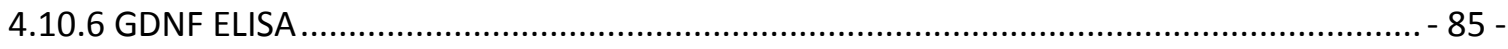

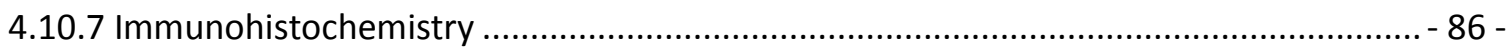

4.11 Dopamine production in cultures of cortical neurons .................................................... 88 - 


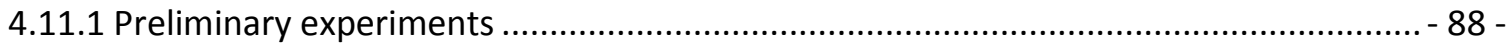

4.11.2 Protein expression upon viral transduction .................................................. - 88 -

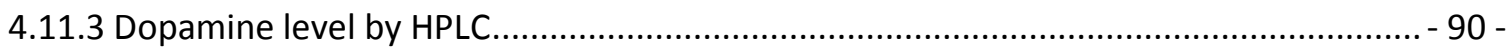

4.11.4 Doapmine and $\alpha$-synuclein interaction ...................................................... 92 -

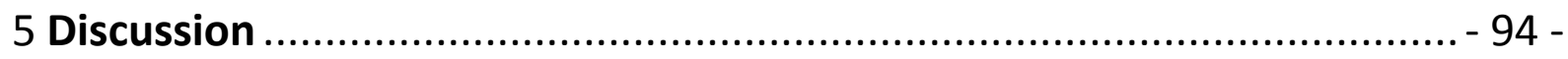

5.1 Development of a AAV vector based regulatable system for use in the CNS ................... 94 -

5.2 GDNF expression in the 6-OHDA rat model .......................................................... 98 -

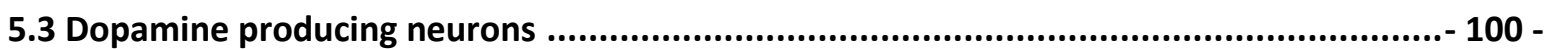

5.4 Considerations about AAV transduction in the CNS ............................................. $101-$

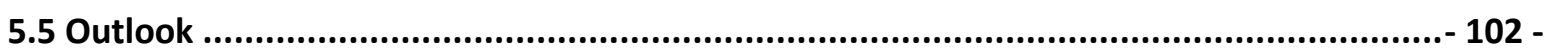

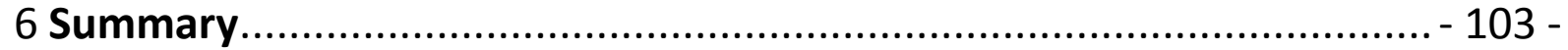

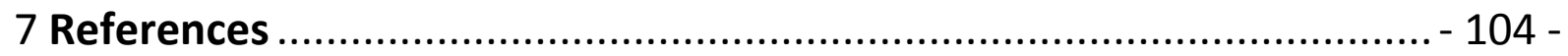

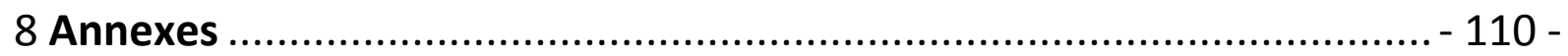

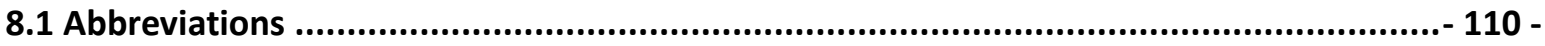

8.2 Curriculum vitae......................................................................................... 113 -

8.3 Publications ........................................................................................... 115 -

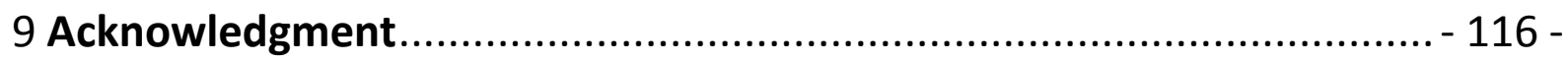




\section{Introduction}

\subsection{Neurodegenerative disorders}

One of the major causes of disability in the western world is the degeneration of the nervous system that may occur for spontaneous or inherited causes, or for traumatic lesions.

The term neurodegenerative disease (NDD) refers to all those diseases in which a loss of neurons is observed. The most common of these diseases are Alzheimer's disease, Parkinson's disease (PD), multiple sclerosis, and prion diseases. The majority of human NDD is not related to inherited mutations of specific genes and develops as a multifactorial pathology associated with aging. In these diseases neuronal cell death happens through various endogenous pathways which are trigged by different insults to cells like accumulation of mis-folded proteins, presence of reactive oxygen species (ROS), mitochondrial complex inhibition, loss of calcium homeostasis, exicitotoxicity or lack of neurotrophic support [1]. Despite the progress in ascertaining of several of these mechanisms, a detailed knowledge of the underlying causes of these diseases is not yet available [2].

For most NDDs cures are not available and treatments are mainly symptomatic; new hopes for patients now arise from new strategies including use of gene therapy which is here presented as a possible alternative for the treatment of PD.

\subsection{Parkinson's disease}

\subsubsection{History and symptoms}

Evidences of Parkinson's disease, at present the second most common NDD in the western society, are found already in the ancient Egypt and the first clinical study of the disease date back to 1817 when James Parkinson first published an article on the disease [3]. Milestones on the study of PD are: the discovery of loss of neurons in the substantia nigra in 1919; the hypothesis in 1959, and its confirmation few years later, of the lack of dopamine (DA) in PD patients; and the first successful trial in treating symptomatically the disease using L-dopa performed by Cotzias in the same years [4].

Diagnosis of PD is based on the manifestation of at least 2 out of the 4 classical symptoms of the disease resumed by the acronym TRAP: Tremor at rest, Rigidity, Akinesia or Bradykinesia (slowness of movement) and Postural instability, [5] and to the positive response to the L-dopa treatment $[5,6])$.

\subsubsection{Causes, pathophysiology, treatment and social incidence of PD.}

In 5 to $10 \%$ of the cases PD had been linked to inherited or de novo mutations observed in different genes [7] ( $\alpha$-syn [8], LRRK2 [9], Parkin [10], Pink1 [11], Dj-1 [12] and ATP13A2 [13]) while, in other patients, it has been noted that the disease was associated to the exposure to certain chemical such MPTP (1-metil 4-fenil 1,2,3,6-tetraidro-piridina) or rotenone [14]. Nevertheless, the majority of the casuistry stays unclear and the neurodegeneration has been related to exicitotoxicity, mitochondrial disfunction, oxidative stress, apoptosis or inflammation [15]. PD affects both the peripheral and the central (CNS) nervous system and loss of neurons is observed in several structures: noradregergic neurons in the locus coeruleus, serotonergic in the raphe nuclei, cholinergic nuclei, the anterior olfactory structures and dopaminergic neurons in the substantia nigra pars compacta (SNpc) [16]. As the major impairments for PD patients derive from motor symptoms, and being the SNpc and the nigro-striatal dopaminergic pathway involved in movements, the loss of dopaminergic neurons in this particular region has been the most studied so far. Parkinson's disease is normally not diagnosed 
until the moment when more than $50 \%$ of the neurons in the SNpc and more than $70 \%$ of their projection in the striatum are lost [17]. The hallmark of the disease is the presence, in the surviving neurons, of the so called Lewy bodies, proteinaceous aggregates formed mainly by $\alpha$-synuclein and ubiquitin [18]. It is not clear if these aggregates are toxic for the neurons by themselves or if it is a cell mechanism to store other more toxic species of the protein in a more inert form [19].

Despite intense studies on the disease, available treatments cannot arrest the neuronal loss and are only symptomatic. The main used treatment for PD is based on the administration of the dopamine precursor L-dopa developed by Cotzias [20] ameliorated in the years by co-administration of Carbidopa, to inhibit the L-dopa metabolism in the periphery, MAO-B (monoamine oxidase B) inhibitors (Selegilines) and COMT (Catechol-O-methyltransferase) inhibitors (Tolcapone), to slow down the metabolism of dopamine in the brain or, in alternative, the administration of other dopamine agonist such bromocriptidine, Ropinirole or Pramipexole [21], [4]. Another alternative treatment consists of the deep brain stimulation technique in which one or more electrodes are implanted in the basal ganglia in order to replace the role of lost neurons. This technique has provided good results but it is not possible to be applied in all the cases [22].

As the neuronal loss cannot be arrested, the dosage of L-dopa have to be increased with the time leading to the onset of side effects such dyskinesia which is defined as involuntary movements at rest that appears upon L-dopa administration [5] and it is thought to be due to fluctuations of dopamine level in the brain.

At present, in the western world, is estimated that Parkinson's disease affects $0.3 \%$ of the total population and about $1 \%$ of the population over 60 years of age [23].

The average economical impact of PD in Europe accounts to $7.500 €$ per patient per year meaning that, being more than 1.1 million cases registered, the costs raises up to nearly 9 billion $€$ per year. Considering that the average age is increasing, meaning a larger number of patients, the cost for the society becomes highly significant [24].

As a consequence of this increase in costs and social impact, alternatives to the L-dopa based treatments are highly demanded. Several cell- and gene therapy based approach are under study and here we explore the possibility to administrate, through an adeno associated viral vector delivery tool and in a controllable way, the Glial Derived Neurotrophic Factor (GDNF) with the aim to protect surviving dopaminergic neurons and, in this way, arrest or slow the course of the disease.

\subsubsection{Gene therapy for Parkinson's disease treatment}

Viral vector based gene therapy consists in the introduction of foreign DNA into target cells by mean of viral vectors delivery tools. In the field of CNS disorders PD has received great attention from this branch of study due to the fact that the disease is routinely (for deep brain stimulation) treated by surgery which rend the viral infusion protocol easier to perform. Furthermore, several animal models mimicking the loss of DA neurons are available for testing the viruses [25].

Neurotrophic factors (NFs), first described by Nobel price's laureate Montalcini [26], is a class of proteins with a pro-survival role for neurons; one of the members of this class of proteins is GDNF, a 211 amino acid protein encoded by the Gdnf gene. The pro-survival role for DA neurons showed by 
GDNF was first described by Lin et al. in 1993, but, despite 20 years are passed, its exact mechanism of action is still not fully understood and the protein seems to act through different receptors such GFR $\alpha 1$, NCAM and RET to promote different pathways like, for example, the ERK/MAPK or the PI3K/Akt [27, 28].

A potential use of GDNF for PD treatment has been recognized since its discovery but, due to the fact that it cannot easily cross the BBB (Blood Brain Barrier) [29], other routes of administration different from diet or peripheral injection had to be defined. Two examples are: a) direct intraputamen infusion of GDNF and b) overexpression of GDNF directly in the brain though injection of a viral vector encoding for the protein. The ability of GDNF to restore motor functionality in, MPTP (1methyl-4-phenyl-1,2,3,6-tetrahydropyridine) or 6-OHDA (6-hydroxydopamine), rodents and nonhuman primate, models of PD has been deeply demonstrated with GDNF administrated both by direct infusion [30,31] or by viral vectors delivery with lentiviruses [32], Adenoviruses [33] and adeno associated viruses [34]. Both direct infusion and viral mediated overexpression of GDNF have reached phase I and I/II clinical trial for PD treatment.

Results from direct infusion of GDNF in clinical trials are controversially debated; an open-label phase I trial [35] showed good recovery of motor skills in patients but the subsequent phase $1 / \mathrm{II}$, randomized and double -blind clinical trial [36], did not confirm these results. While a open-label phase I clinical trial for GDNF delivery through AAV2 has been recently approved [37] and it is now in a recruiting phase, a similar treatment was performed using neurturin (NTN), a homolog of GDNF. A phase I open-label clinical trial showed good recovery in the motor function of patients [38] but these results could not be confirmed in a phase II double-blind, randomized, controlled, trial where, moreover, several side effects were observed [39].

It has been hypothesized that the lack of therapeutic effect might be due to the fact that selected patients were all in an advanced stage of the disease with the consequence that few DA neurons were still present; the NF, thereafter, did not have a target to act. Adverse side effects might have derived from a mis-targeting of the catheter/virus or to a too high GDNF expression when viruses were used. Moreover, studies on animal models have shown that a mis-targeting or an overexpression of GDNF cause three major side effects in tested animals: loss of weight [40] probably due to an unspecific action of the NF in other areas of the brain (for example the hypothalamus), aberrant sprouting of neurons [41] which might be due to a too high level of expression in neurons that normally express it only at low level, and downregulation of tyrosine hydroxylase (TH) the rate limiting enzyme for dopamine's synthesis [42].

In this project we propose to combine the use of AAV with a temporal regulatable promoter for GDNF expression in order to $A$ ) have the possibility to silence the expression of the transgene in case of unwanted side effects and B) as a consequence, increase the safety level of the treatment in order to promote its use in less advanced patients where GDNF could have a stronger impact in treating the symptoms.

\subsection{Viral vectors as tools in gene therapy}

Viral vectors are modified wild type viruses that allow the delivery of desired DNA sequences into living cells. The first successful attempt in modifying a wild type virus date back to 1976 when Paul Berg used a modified SV40 virus (Simian vacuolating virus 40 ) to transduce monkey kidney cells in 
culture [43]. Since then a great number of viral vectors have been developed starting from different wild type viruses and their use includes tracking proteins or cells, study proteins function by gain or loss of function experiments, and for therapeutic purpose. The first use of a viral vector for therapeutic purpose in human (gene therapy) date back to 1990 [44] and since then over 1700 clinical trial have been performed with different degree of success (Gene Therapy Clinical Trial database www.wiley .com) [45].

One of the most widely used viruses for gene therapy is based on adeno associated virus (AAV).

\subsubsection{Adeno associated vectors}

Adeno associated viruses are small dependoviruses from the Parovidiae family; as dependovirus it is not able to replicate without the presence of a helper virus such as Adenovirus, Herpes virus or Vaccinia virus. The wild type AAV genome is formed by a $\sim 4700$ bp single strand DNA sequence flanked by two inverted terminal repeats (ITRs). Between the 2 ITRs two non overlapping open reading frames (ORF) are present (figure 1); the rep gene encodes for four proteins responsible for self transcription and integration into the host genome while the cap gene encodes for 3 capsid proteins.

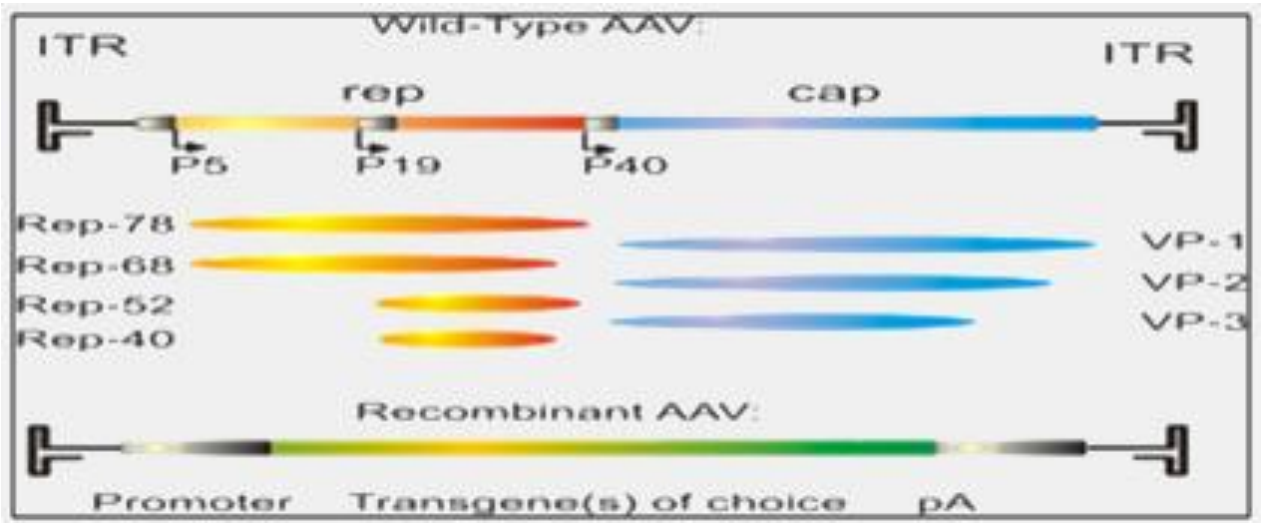

Figure 1) Representation of a wild type AAV genome, the expressed protein, and the recombinant version of the vector. Rep = protein responsible for viral replication and integration/excision from the genome; Cap = protein for the capsid; P5, P19, P40 = promoters; PA =poli adenilation sequence; ITR = inverted terminal repeats.

Wild type AAVs are not associated with any disease in mammals and they are able to transduce both dividing and non dividing cells; due to their safety and transduction properties these viruses have received great attention from the scientific community in order to generate recombinant viruses to be used for gene therapy in the CNS [46].

The first recombinant AAV vector was generated by Samulski et al in 1985 [47]; he assessed that only the two ITRs are necessary for the encapsidation of the genome while all the other proteins can be provided in trans during virus production. This characteristic allows the substitution of $95 \%$ of the original genome (except for the ITRs) with a DNA sequence of interest. Despite nearly all the genome can be substituted, a drawback of AAVs is its limited DNA packaging capacity of $\sim 4800 \mathrm{bp}$. As the rep proteins are deleted, viral DNA is not able to integrate in the host genome, and persists in the nucleus as episomal concatemer. Moreover AAVs have been better characterized and new serotypes have been discovered. To date 11 serotypes are available and more than 100 variants are known. [48]. Different serotypes show different transduction properties in term of cell tropism (by recognizing different receptors), immunological reaction and expression level of the transgene. The immunological aspect is particularly important due to the fact that AAV2 is common in nature and 
most of the people have been in contact and have developed antibodies against it. [49].The availability of different serotypes, thereafter, gives the possibility to circumvent a possible immunological reaction.

In summary advantages of recombinant AAV for the use in the CNS are:

$\checkmark$ Ability to infect dividing and not-dividing cells

$\checkmark$ Not associated with any disease

$\checkmark$ Availability of different serotypes

$\checkmark$ Efficient gene transfer

Drawbacks are:

* Restricted DNA capacity

* Possible immune reactions

At present, around 90 clinical trials are on-going using AAVs as a delivery system 14 of which are directed to the CNS (www.wiley.com). Treated diseases include Parkinson Disease, Alzheimer disease, San Filippo syndrome, amyotrophic lateral sclerosis and epilepsy.

\subsubsection{Regulated gene expression system}

A characteristic of gene therapy, especially when applied for targeting post-mitotic cells in the CNS, is its intrinsic irreversibility; this feature is advantageous because its employment requires only one application but it is disadvantageous if side effects arise. A regulatable system to overcome this drawback would be therefore highly recommendable to further promote gene therapy in clinical trials.

Regulated gene expression systems for gene therapy purposes should own certain features [50,51]:

$\checkmark \quad$ Ideal "zero" basal level of expression in the non induced state, or at least, a level without biological effects

$\checkmark$ Biologically relevant expression in the induced state

$\checkmark$ Constituted by elements of human origin

$\checkmark$ Positively induced

$\checkmark$ Demonstrated safety profile of the inducer in human

$\checkmark$ Dose responsiveness

$\checkmark$ Fast kinetic

$\checkmark$ Repetitiveness of induction

$\checkmark$ No pleiotropic effects

$\checkmark$ Not affecting endogenous gene expression

$\checkmark$ be region or cell specific

Regulated gene expression systems can be divided in different categories depending on the nature of the activator, which can be a small ligand (table 1), an endogenous stimulus or a physical agent.

The most common ligand-based gene regulatable system is the Tetracycline-Controlled Transcriptional Activation system called tet-off or its alternative tet-on system. Both systems consist of two cassettes: the first cassette expresses the Escherichia coli tet repressor protein (tetR) fused with the VP16 activation domain while the second cassette consists of a tet-operator DNA sequence (tetO) followed normally by a CMV (cytomegalovirus) promoter and the gene of interest (GOI). The 
binding of tetR to tetO, which promote the transcription of the $\mathrm{GOI}$, depends on the conformational state of tetR that, in turn, depends on the presence of the antibiotic tetracycline (tc) or its homologue doxycyclin (dox). In the tet-off system the presence of the antibiotic prevents the binding and the transcription while in the tet-on system, due to a 4 AA substitution, the opposite effect is observed. The affinity of the antibiotic in the mutated form (tet-on) is 100 fold lower than the original one but new versions of this protein have been developed to overcome this handicap such, for example, the tet-on 3G [52]. More improvements on the tet systems to optimize the induction and lower the background expression were done by mutating the CMV [53].

The tet systems is the most widely employed gene regulatable expression system and it has been used for different purposes including for GDNF delivery in the brain. Manfredsson et al described the use of a single AAV2/5 vector with the tet-off system in unlesioned rats using doxycycline as drug to silence the transgene expression. No leakiness in the expression in the off-state was observed and the TH downregulation and the loss of weight due to GDNF overexpression were avoided when the system was off [54]. Chtarto et al. studied the tet-on system packed in a single AAV1 vector and dox was used as inducer; the virus was injected in unlesioned animals and the GDNF level was evaluated by IHC and ELISA revealing a nearly undetectable level of expression in the off-state and a 15 folds GDNF overexpression ( $60 \mathrm{pg} / \mathrm{mg}$ tissue) comparing to the endogenous level (4 pg/mg tissue) in the on-state. The inclusion of the transcription enhancing element WPRE affected negatively the offstate without giving any beneficial effect in the on-state [55]. Georgievska et al. used a double Lentiviral (LV) vector system with the tet-on inducible promoter to express GDNF under dox control. A low but detectable GDNF expression in the off-state was observed which could be avoided by lowering the dosage of the viruses [56]. Yang used a single AAV1 vector with the tet-on system in 6OHDA lesioned rats to express GDNF; no leaking in GDNF expression in the off-state was observed and animals showed a partial motor recovery when the system was activated [57]. Liu et al. injected a single AAV1 vector with the tet-on system in the cochlea to protect it from aminoglycoside-induced damage. No leakiness was observed in the off-state and kanamicin induced animals significantly recovered [58].

The tet-off system, compared to the tet-on system, displays better compromises between the on and off states but the inducer has to be provided to silence the gene expression, a characteristic non compatible for a possible application in clinical trials. Moreover concerns about the tet systems arise due to the bacterial origin of its components which might trigger an immunological response when used in clinical trials. Subretinal injection of AAV vectors expressing the tet-on system in non human primate displayed a repetitiveness of induction over a period of 5 years without any sign of immune response[59]; on the contrary, intramuscular injection of Adenoviral vector carrying the tet systems leaded to both humoral and cellular mediate immune response resulting in rapid loss of tet-mediated control of transgene expression [60]. This difference in immune response can be explained by the fact that the retina, like the brain, is an immune-privilege site [61].

The rapamycin inducible system is based on the dimerization of two proteins upon addition of rapamycin; the first protein is formed by a hybrid zinc finger DNA binding domain coupled with Fk506 binding domain while the second chimeric protein is constituted by the FKBP rapamycin binding domain (FRAP) and the C-terminus of the p65 activation protein. Once dimerized, these two proteins can promote the transcription of a $\mathrm{GOI}$ from a promoter containing the ZFHD1 binding sequence [62]. This system is entirely constituted by human components and the drawback given by the potent immunosupressor activity of rapamycin has been overcome by using analogs of it. Despite these 
advantages this system has been only poorly used. Hadaczek et al. injected a double AAV2 vector system containing the rapamycin inducible promoter for GDNF expression in rat striatum and evaluated dosages and route of rapamycin delivery obtaining a good expression ratio ( $\sim 50$ folds) between the on- and the off-state but with a consistent leakage in GDNF expression in the non induced state which resulted to be 20 folds higher comparing to the endogenous GDNF expression level [63].

Another inducing system makes use of the steroid ecdysone; in this case, the truncated ecdysone receptor (EcR) is fused with the VP16 activation domain. In presence of the steroid the receptor changes conformation and became able to bind to the ecdysone responsive element (ECRE) promoting the transcription of the GOI [64]. This system has the advantage that is not responsive to any mammalian hormones but has the drawback of being constituted of non-mammals element which might be immunogenic. Moreover the inducing drug ecdysone, and its homologue muristerone A, have not been approved from FDA (Food and Drug Administration) for use in humans. This system is mainly used in vitro and studies are ongoing for promoting its application in mammals [65].

\begin{tabular}{|c|c|c|c|c|c|c|}
\hline Vector & Target & $\begin{array}{l}\text { Switch } \\
\text { System }\end{array}$ & inducer & leakiness & comments & References \\
\hline $\begin{array}{l}\text { AAV2/5 } \\
\text { single } \\
\text { vector }\end{array}$ & $\begin{array}{c}\text { Rat } \\
\text { substantia } \\
\text { nigra }\end{array}$ & Tet-off & $\begin{array}{c}\text { Dox } \\
20 \mathrm{mg} \text { to } 3 \mathrm{~g} / \mathrm{kg} \\
\text { food }\end{array}$ & no & $\begin{array}{c}\text { Side effects } \\
\text { avoided when } \\
\text { system was off }\end{array}$ & [54] \\
\hline $\begin{array}{l}\text { AAV1 } \\
\text { single } \\
\text { vector }\end{array}$ & $\begin{array}{c}\text { Rat } \\
\text { striatum }\end{array}$ & Tet-on & $\begin{array}{c}\text { Dox } \\
600 \mu \mathrm{g} / \mathrm{ml} \\
\text { drinking water }\end{array}$ & yes & $\begin{array}{c}\text { WPRE affects } \\
\text { negatively the off- } \\
\text { state }\end{array}$ & [55] \\
\hline $\begin{array}{c}\text { LV } \\
\text { double } \\
\text { vector }\end{array}$ & $\begin{array}{c}\text { Rat } \\
\text { striatum }\end{array}$ & Tet-on & $\begin{array}{c}\text { Dox } \\
1 \mathrm{mg} / \mathrm{ml} \\
\text { drinking water }\end{array}$ & yes & $\begin{array}{c}\text { Leakiness could be } \\
\text { avoided by } \\
\text { decrease viruses } \\
\text { dosage }\end{array}$ & [56] \\
\hline $\begin{array}{l}\text { AAV1 } \\
\text { single } \\
\text { vector }\end{array}$ & $\begin{array}{c}\text { Rat } \\
\text { striatum }\end{array}$ & Tet-on & $\begin{array}{c}\text { Dox } \\
600 \mu \mathrm{g} / \mathrm{ml} \\
\text { drinking water }\end{array}$ & no & $\begin{array}{l}\text { Only partial motor } \\
\text { recovery }\end{array}$ & [57] \\
\hline $\begin{array}{l}\text { AAV1 } \\
\text { single } \\
\text { vector }\end{array}$ & Rat cochlea & Tet-on & $\begin{array}{c}\text { Kan } \\
333 \mathrm{mg} / \mathrm{kg} \\
\text { twice per day } \\
\text { for } 12 \text { days }\end{array}$ & no & $\begin{array}{c}\text { Study about } \\
\text { leakiness not clear }\end{array}$ & [58] \\
\hline $\begin{array}{l}\text { AAV2 } \\
\text { double } \\
\text { vector }\end{array}$ & $\begin{array}{c}\text { Rat } \\
\text { striatum }\end{array}$ & $\begin{array}{l}\text { Rap } \\
\text { based }\end{array}$ & $\begin{array}{c}\text { Rap } \\
\text { IP: } 1,3,10 \mathrm{mg} / \mathrm{kg} \\
\text { for } 1 \text { or } 3 \text { days } \\
\text { Oral: } 1 \text { or } 3 \times \\
0.5 \mathrm{mg} / \mathrm{kg} \\
\text { CED: } 2 \times 50 \mathrm{ng}\end{array}$ & low & $\begin{array}{l}\text { Different route of } \\
\text { rap administration }\end{array}$ & [63] \\
\hline
\end{tabular}

Table 1) Summary of studies employing regulatable system to express GDNF in the brain. Dox = doxyciclin; Kan = kanamycin; Rap = rapamycin; IP = intraperitoneally; CED = convection-enhanced delivery; AAV = adeno associated virus; LV = Lentivirus.

A different concept for inducible proteins expression is given by the conditional promoters; in these cases the transcription of the $\mathrm{GOI}$ is trigged by environmental factors. The most common example is the integration of the hypoxia-response elements (HRE) DNA sequence in proximity of the GOI; in hypoxic conditions, such in ischemia or tumor growth, the transcription of the $\mathrm{GOI}$ is activated 
together with hypoxic genes [66]. Similarly, the antioxidant response element (ARE) can be used; in this case the transcription of the $\mathrm{GOI}$ is associated with oxidative stress, a condition present in PD [67].

The control of protein expression may happen at post translational level as well: in this case a destabilizing domain that drives the protein to proteosome-mediated degradation is added to the protein. The degradation of the constitutively expressed protein can then be suppressed by addition of a stabilizer [68].

Referring to protein regulated expression systems regulated by a small ligand the most important features, for a possible application in human, can be summarized as:

- the tet-on system displays a good regulation but a detectable background expression in the off-state;

- the tet-off system shows a good regulation and a undetectable background expression in the off-state;

- both tet-system are immunogenic when applied in non-human primate muscle;

- the rapamycin based system shows good induction but a high background expression in the off-state;

- the ecdysone system is based on non-mammals elements.

In this project we propose, as alternative to the fore mentioned systems, the adoption of the pSwitch ${ }^{\mathrm{TM}}$ gene expression system which is mainly constituted by elements of human origin and it is positively induced by the steroid mifepristone.

\subsubsection{The pSwitch system}

The GeneSwitch ${ }^{\text {TM }}$ system (here called pSwitch) is a steroid-based gene inducible expression system developed in 1994 by Wang et al. [69]. Despite some promising results (presented later in the chapter) the system has never been widely used and, in the last decade, it has been studied only sporadically.

The pSwitch system is formed by three essential elements: a regulation cassette, a responsive cassette, and the steroidal inducer mifepristone (MF).

The original regulation cassette of the GeneSwitch ${ }^{\mathrm{TM}}$ system encodes for the chimeric protein pSwitch under the control of a hybrid promoter. The promoter is constituted by a GAL4 UAS (Upstream Activating Sequence) that consist of 4 repetitions of a 17 nucleotides sequence; each of this repetition can be recognized by a GAL4 DBD (DNA Binding Domain). The second part of the promoter consists of a Herpes Simplex Virus thymidine kinase (TK) minimal promoter (ptk) that allows the expression of the pSwitch protein. The 654 amino acid (AA) $(73 \mathrm{kDa})$ chimeric protein pSwitch is formed by three distinct domains: the GAL4 DBD, the truncated human progesterone receptor ligand binding domain (hPR-LBD) and the human NF-KB p65 activation domain. The Saccharomyces Cerevisiae GAL4 protein regulates the transcription of target genes by binding as a homodimer to the 17 nucleotides GAL4 sequences forming the UAS of target genes. The pSwitch fusion gene encodes for AA 1-93 of this protein which includes the region responsible for DNA binding (AA 1-65) [70], dimerization (AA 65-93) [71] and nuclear localization (AA 1-29) [72]. The second domain of the pSwitch protein consists of a truncated human progesterone receptor ( $h P R-L B D)$; the full length $h P R$ 
is a transcription factor responsive to progesterone and other analogues that, in presence of a ligand, dimerizes and promotes the transcription of target genes. The ligand-binding function is localized in the C-terminal portion of the protein (AA 640-933) [73]. Through directed mutagenesis it was discovered a truncated form (hPR-LBD; AA 640-914) which can still bind to the progesterone analogue mifepristone (MF) but has lost its affinity for the human progesterone. This last fragment is used in the pSwitch protein [69].The last domain of the chimeric protein consist of the AA 283-551 of the human p65 protein in which two distinct transactivation domains have been identified [74]. Human $\mathrm{p} 65$, together with $\mathrm{p} 50$, forms the second messenger NF-KB heterodimer and function as a pleiotrophic transcriptional activator in eukaryotes [75]. A last element in the regulation cassette is the synthetic intron IVS8 placed between the promoter and the pSwitch protein to enhance the transcription.

The responsive cassette is responsible for the transcription of the gene of interest (GOI) which is promoted by a hybrid promoter formed by 6 repetitions of the already mentioned GAL4 UAS linked to a TATA box sequence from the adenovirus major late E1b gene [76]. Similarly to the regulation cassette an IVS8 intron is placed between the promoter and the GOI to enhance the transcription.

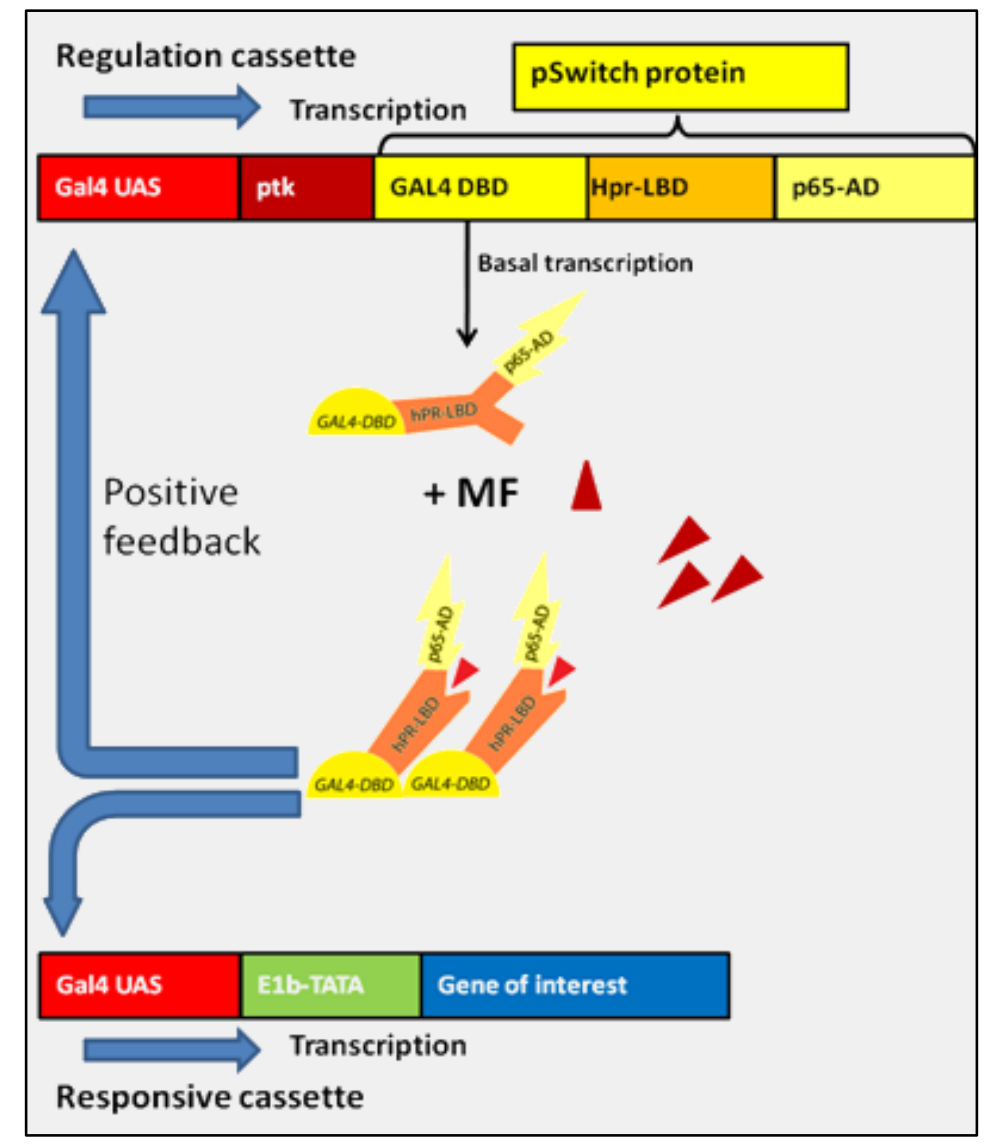

Figure 2) Mechanism of action of the GeneSwitch(TM) system. The pSwitch protein is expressed at a basal level by the regulation cassette and stay in its inactive monomeric form; upon addition, MF binds to the hPR-BDL forcing the protein to change conformation and to dimerize. The dimer can then bind to the GAL4 UASs in the responsive cassette promoting the transcription of the gene of interest. Moreover the chimeric protein can bind to the GAL4 UAS in the regulation cassette enhancing the transcription of its own gene in order to obtain a positive feedback loop. MF = mifepristone; UAS = upstream activatin sequence; ptk = Herpes Simplex Virus thymidine kinase (TK) minimal promoter; Hpr-LBD = human progesterone receptor ligand binding domain; p65 AD = p65 human activation domain; E1b-TATA = adenovirus major late E1b gene TATA box. 
The last element of the GeneSwitch ${ }^{\mathrm{TM}}$ system is the inducing agent RU486 or Mifepristone (11ß-[4dimethylamino] phenyl-17 $\beta$-hydroxy-17-[1-propynyl]estra-4,9-dien-3-one) a synthetic 19-norsteroid that binds with high affinity to the human progesterone receptor and to the glucocorticoid receptor (both $\mathrm{k}_{\mathrm{d}}<1^{*} 10^{-9} \mathrm{M}$ ) acting as an antagonist [77]. MF has been approved for use in humans as a component for medical termination of pregnancy ( $10 \mathrm{mg} / \mathrm{kg}$ ), for contraception ( 0.02-0.2 mg/kg), for treatment of breast and ovarian cancer ( $1 \mathrm{mg} / \mathrm{kg} /$ day), for HIV treatment (Clinical trial phase I/II 1-4 mg/kg) [78], and for major depression (10 mg/kg)[79].

It has been shown that MF is partially able to cross the BBB in rats being its concentration in this organ $\sim 28 \%$ comparing to the one in the serum [80].

The GeneSwitch ${ }^{\mathrm{TM}}$ system requires the presence in the same cell of both cassettes which can be introduced by transfection, or by viral transduction.

When the system is in its off-state the transcription of the GOI from the responsive cassette should be silenced while the regulation cassette should express the pSwitch protein at a basal level. In this stage the pSwitch protein stays as an inactive monomer and accumulates in the nucleus. Upon addition, MF binds to the hPR-LBD causing a change of conformation and consequent dimerization of the protein that, in turn, become able to bind to the GAL4 UAS promoting the initialization of the transcription of the GOI. The activated protein promotes a positive feedback loop by binding to the GAL4 UAS in the regulation cassette enhancing the transcription of its own gene.

Once the MF is removed or metabolized the pSwitch dimers dissociate turning the system off (figure 2).

After its development, the pSwitch system has been employed in several studies but, as mentioned, in the last decade it has been used only rarely.

The pSwitch system has been used to generate transgenic mice lines with the pSwitch protein under the control, for example, of a liver specific promoter [81], brain specific promoter [82, 83] or to generate knock-out mice using the system in combination with the CRE recombinase [84]. Due to the fact that several transgenic lines of drosophila already express genes under the control of GAL4 UAS this animal was further engineered to express the pSwitch protein in order to obtain genes under spatial and temporal control [85]

Plasmid-based vectors containing the pSwitch system have been used to transfect mouse muscles and express EGFP [86], secreted human placental alkaline phosphatase (SEAP) [87], or erythropoietin [88].

The pSwitch system has been used in combination with viral vectors as well; Burcin et al. substituted the UAS ptk promoter with a liver specific promoter to control the expression of the human growth factor (hGF). The two cassettes were then placed in a unique plasmid which was packed in an adenoviral vector. The study shows that mice infected by tail vein injection express repetitively the hGF upon MF administration [89].

The only study involving the use of the pSwitch system in association with a viral vector delivered in the brain was performed by Oligino et al; in this case the pSwitch protein was expressed under the control of the human cytomegalovirus immediate-early promoter (hCMV IEp) while the GOI in the responsive vector was LacZ. The two cassettes were placed in a unique plasmid and packed in a 
Herpes Simplex Virus (HSV) which was stereotaxically injected in rat hippocampus. Upon intraperitoneal MF injection ( $25 \mathrm{mg} / \mathrm{kg} /$ day for 2 days) a 150-fold increase of LacZ was observed with a really low basal level of expression in not induced animals [90].

The aim of this study is to demonstrate that, in the CNS, the pSwitch system might provide a valid alternative to the widely used tet-on systems with the further advantage of being constituted mainly by human elements which might render this system less immunogenic in mammals comparing to the tet-on system.

\subsection{Animal model of PD}

PD is subject of extensive study: studies range from the in vitro scale to the employment of nonhuman primates. Aggregation properties of $\alpha$-syn and role of other proteins are studied in cell free systems, in yeast, in dissociated cell cultures and in different animals: Drosophila, C.Elegans, Zebrafish, Medaka fish, rodents and primates. The focus of this chapter will be only in the animal models with particular attention to rodents [91].

No other species in nature apart of human is known to develop the Parkinson's disease; thereafter, in the last 40 years, researchers have developed several animal models of the disease using different approaches. Animal models of PD can be divided in two main categories: toxin induced and genetically induced. Both categories (and subcategories) have advantages and disadvantage and the model has to be chosen depending on the pursued objective.

Genetic models of PD have been conceptualized after the discovery of various mendelian form of the disease starting from the mid ' 90 s with the description of $\alpha$-syn and its mutated forms in certain familiar cases of the disease [8]. Since then many other mutations have been found in other proteins and different animal models expressing the wild-type or mutated proteins have been developed either by generating transgenic animals or by overexpressing proteins by mean of viral vector transduction. Transgenic animals include Drosophila [92] and rodents [93, 94]. Mutated or overexpressed proteins include $\alpha$-syn [95, 96], LRRK2, Parkin, Dj-1, PINK1. A different approach had been used in the generation of the MitoPark mouse in which mitochondrial function is selectively impaired in dopaminergic neurons by knocking down Tfam, a gene not directly related to PD [97].

PD-like syndrome can be generated in animals using different toxins; the first toxic compound for the nigrostriatal pathway studied in the mid '80s was MPTP (1-Methyl-4-phenyl-1,2,3,6tetrahydropyridin); this molecule, once crossed the $\mathrm{BBB}$, is converted to $\mathrm{MPP}^{+}$(1-methyl-4phenylpyridinium) by MAO-B (monoamine oxidase-B) and transported inside DA neurons by DAT (dopamine transporter) where it blocks mitochondrial complex I activity, thereby, killing selectively DA neurons [98]. The discovery that environmental factors can trigger the onset of the disease leaded to the discovery of other compounds such paraquat and rotenone.

Another model consists in the infusion in the nigrostriatal pathway of the bacteria endotoxin lipopolysaccharide (LPS) which promote microglia activation and subsequent loss of DA neurons [99].

The first [100], and, in the last 40 years, one of the most employed models of PD in rodents involves the infusion in the nigrostriatal pathway of the neurotoxin 6-hydroxidopamine (6-OHDA). 6-OHDA is a hydroxylated analogue of dopamine which is transported selectively inside DA neurons by DAT. Once inside neurons this toxin acts as a pro-oxidant promoting a high rate of hydrogen-peroxide 
formation in the cytosol leading to neuronal death. Moreover 6-OHDA accumulates in mitochondria where it inhibits mitochondria complex 1 activity. This molecule is not able to cross the BBB and thereafter it has to be infused directly in different areas of the brain: the SN (substantia nigra), the medial forebrain bundle, or the striatum. Depending on the amount of the applied drug and on the coordinates of injection, a different, but reproducible, size of DA neurons lesion can be obtained. The toxin is normally injected unilaterally in the brain and the contra-lateral hemisphere is kept as a control. Two advantages derive from this protocol: first, a bilateral lesion is often lethal or anyway impairs severely the locomotor functions of animals that will then need particular care. Secondary, a unilateral lesion of the dopaminergic system leads to a proportional motor asymmetry in animals which can be measured by appropriate tests to evaluate the magnitude of it [91]. These tests include the apomorphine induced rotations, the corridor tests and the cylinder tests (See materials and methods).

\subsection{Aim of the project}

One of the main reasons why gene therapy treatments are only slowly accepted for clinical trials is their intrinsic irreversibility especially if they are applied for CNS disorders. Several studies in animal models of PD have shown the great potential in the use of neurotrophic factors to treat this devastating disease. Due to the high risk profile of gene therapy only patients with advanced PD were selected for viral mediated delivery of NFs leading to the hypothesis that the low success rate was due to the lack of target neurons where the NFs can act. A way to circumvent the irreversibility drawback is to gain the control of gene expression upon administration of an inducer which has to be well tolerated by the patient. In case of unwanted side effects the inducer can be withdraw blocking the expression of the transgene. Moreover the switching system should be able to undergo to several cycles of activation-deactivation and the level of expression should be dose-dependent with the inducer.

In this thesis we explore, for the first time, the possibility to combine a steroid-based expression system (GeneSwitch ${ }^{\mathrm{TM}}$ ) and the adeno associated viral (AAV) vector delivery tool in order to express the neurotrophic factor GDNF in the striatum of a 6-OHDA PD rat model under the control of the inducing drug Mifepristone.

The advantage of the pSwitch system is that it is mainly constituted of human elements, and provides an alternative to the most used tet-on system which has shown to trigger an immune response when used in primate muscle.

The first part of the project consists on adapting the GeneSwitch ${ }^{\mathrm{TM}}$ system to the AAV vectors to find the most suitable combination of the two cassettes belonging to the regulatable system and other transcription elements using EGFP as a reporter gene. In this part of the study, levels of EGFP expression are evaluated in the on- and off-states, and the course of its expression is followed over several cycles of activation in the rat striatum or in the same animals by in vivo imaging. Studies on dosages and viral vectors interaction are performed as well.

In a second part of the project a 6-OHDA rat model of PD is established and the effect of short pulses of GDNF expression is compared with the constitutive expression of the NF. 


\section{Material}

\subsection{Chemicals, kits, enzymes and drugs}

Applichem: acrylamide, agarose for gel, ampicillin, boric acid $\left(\mathrm{H}_{3} \mathrm{BO}_{3}\right)$, calcium chloride $\left(\mathrm{CaCl}_{2}\right)$, chloroform, D-(+) glucose, chloroform, cumaric acid, dimethyl sulfoxide (DMSO), EDTA, ethanol absolute, Ficoll, glycerol, glycin, HEPES, isopropanol, kanamicin, potassium chloride (KCl), LB Agar, LB media, Magnesium sulfate $\left(\mathrm{MgSO}_{4}\right)$, methanol, mifepristone, modified Eagle's medium (DMEM), paraformaldeide (PFA), phosphate buffered saline (PBS), potassium chloride ( $K C l)$, phenol equilibrated stabilized, sodium azide, sodium chloride $(\mathrm{NaCl})$, Sodium dodecyl sulfate (SDS), sodium metabisulfite $\left(\mathrm{Na}_{2} \mathrm{~S}_{2} \mathrm{O}_{5}\right)$, Sodium Phosphate $\left(\mathrm{Na}_{3} \mathrm{PO}_{4}\right)$ sucrose, TRIS, Triton $\mathrm{X}$.

Axis Shield: Iodixanol Opti prep.

Bayer: bepanthene.

Biorad: Precision Plus Protein ${ }^{\mathrm{TM}}$ standard.

Calbiochem: sodium citrate, luminal.

Braun: Braunol, histoacrylic glue.

Fermentas: Hind III Lambda DNA.

Fluka: sodium octasulfonic acid, Monopotassium phosphate $\left(\mathrm{KH}_{2} \mathrm{PO}_{4}\right)$, TWEEN 20.

Fresienius Kabi: Ampuwa water.

Gibco: B27 Supplement, DMEM:F12 (1:1), Hank's Balanced Salt Solution (HBSS), Neurobasal medium (NBM), neomicyn, penicillin, sodium bicarbonate solution $7.5 \%$, streptomycin.

Medistar: Ketamine.

Merck: fat milk, HPLC water, hydrogen peroxide $\left(\mathrm{H}_{2} \mathrm{O}_{2}\right)$, magnesium chloride $\left(\mathrm{MgCl}_{2}\right)$, Perchloric acid $\left(\mathrm{HClO}_{4}\right)$, sodium metabisulfite $\left(\mathrm{Na}_{2} \mathrm{~S}_{2} \mathrm{O}_{5}\right)$, trichloroacetic acid $\left(\mathrm{C}_{2} \mathrm{HCl}_{3} \mathrm{O}_{2}\right)$.

New England Biolabs (NEB): 2-Log DNA marker, broad range protein marker, dNTPs.

Novagen: benzonase.

PAA the cell culture company: fetal calf serum (FCS), newborn goat serum (NGS), PS (penicillin/streptomycin), Trypsin for HEK 293 cells.

Pfitzer: ketanest, rymadil.

Riemser: xylarien.

Roche: proteinase inhibitor, glycerol.

Roth: citric acid monohydrate, Mowiol, sodium acetate.

Seromed: L-glutamine.

SerumWerk: methamizol. 
Serva: bromophenol blue sodium salt (BPB).

Sigma: ammonium peroxide sulfate (APS), ascorbic acid, Bactotryptone, Dithiothreitol (DTT), Etidium bromide, L-dopa, mineral oil, TEMED, transferrin, yeast extract.

Teclapharm: apomorphine.

\subsection{Solutions}

6X SDS-Loading buffer: 350 mM Tris- $\mathrm{HCl}$, pH 6.8; 10\% SDS, 0.6 M DTT, 30\% Glycerol, 0.03\% BPB.

40\% PFA in PBS: for 2 liters preparation dissolve $80 \mathrm{~g}$ of PFA and $19.10 \mathrm{~g}$ of PBS in 1.8 litres of bidistilled water; add few drops of $1 \mathrm{M} \mathrm{NaOH}$ and heat the mixture to $60^{\circ} \mathrm{C}$ under stearing till it is clear. Cool it down on ice, $\mathrm{pH}$ at 7.4, bring the volume to 2 liters with bi-distilled water and filter with through Whatman $5951 / 2$ folded filters. Store at $4^{\circ} \mathrm{C}$.

Annealing buffer: $20 \mathrm{mM}$ Tris, $\mathrm{pH} 7.8,100 \mathrm{mM} \mathrm{NaCl}$, and $0.2 \mathrm{mM}$ EDTA.

CMV medium: HBSS medium diluted with bi-distillede $1: 10$ and $\mathrm{pH}$ adjusted to 7.4 with sodium bicarbonate solution $7.5 \%$.

Citric saline: $135 \mathrm{mM}$ potassium chloride, $15 \mathrm{mM}$ sodium citrate.

DNA loading buffer (6x): 15\% Ficoll 400 DL, 100 mM LiCl, 2\% glycerol, 100 mM EDTA, pH 8.0; 0.6\% SDS, $0.03 \%$ BPB in $\mathrm{H} 2 \mathrm{O}$.

ECL-1 (for $10 \mathrm{ml}$ solution): $100 \mu \mathrm{l} 250 \mathrm{mM}$ luminal, $44 \mu \mathrm{l} 90 \mathrm{mM}$ Cumaric, $1 \mathrm{ml} 1 \mathrm{M}$ Tris $\mathrm{pH} 8.5$ and water to $10 \mathrm{ml}$ final volume.

ECL-2 (for $10 \mathrm{ml}$ solution): $6 \mu \mathrm{l} \mathrm{30 \%} \mathrm{H}_{2} \mathrm{O}_{2}, 1 \mathrm{ml}$ Tris pH8.5 and $9 \mathrm{ml} \mathrm{H}_{2} \mathrm{O}$.

Freezing solution: $65 \%$ glycerol, $100 \mathrm{mM} \mathrm{MgSO}_{4}, 25 \mathrm{mM}$ tris; $\mathrm{pH} 8.0 \rightarrow$ autoclave.

HCN medium: $5 \mu \mathrm{g} / \mathrm{ml}$ transferrin, PSN (Penicillin $50 \mu \mathrm{g} / \mathrm{ml}$, Streptomycin $50 \mu \mathrm{g} / \mathrm{ml}$, Neomycin 100 $\mu \mathrm{g} / \mathrm{ml}$ ), $2 \mathrm{mM}$ L-Glutamin, 2\% B-27 supplement in Neurobasal medium (NBM).

HeBS (2X): $280 \mathrm{mM} \mathrm{NaCl}, 10 \mathrm{mM} \mathrm{KCl}, 1.5 \mathrm{mM} \mathrm{Na} 2 \mathrm{HPO} 4,12 \mathrm{mM}$ glucose, $50 \mathrm{mM}$ HEPES, in $\mathrm{H}_{2} \mathrm{O}, \mathrm{pH}$ $7.04 / 7.05$ sterile filtered and stored in aliquots at $-20^{\circ} \mathrm{C}$.

HPLC buffer (2 I): in $1.6 \mathrm{ml} \mathrm{HPLC}$ water add $13.946 \mathrm{~g}$ sodium acetate, $14.72 \mathrm{~g}$ citric acid monohydrate, $0.21 \mathrm{~g}$ sodium octasulfonic acid, $0.096 \mathrm{~g}$ EDTA; stear and $\mathrm{pH}=4.3$ using $1 \mathrm{M}$ citric acid. Add $210 \mathrm{ml}$ methanol and bring volume to 2 I with HPLC water $\rightarrow$ filter and degas.

Lysis buffer for WB ( $1 \mathrm{ml}$ solution): $840 \mu$ l sterile $\mathrm{H}_{2} \mathrm{O}, 50 \mu \mathrm{l} 1 \mathrm{M}$ Tris pH 8.0, $50 \mu \mathrm{l} 10 \%$ SDS, $50 \mu \mathrm{l} 20 \mathrm{X}$ proteinase inhibitor and $10 \mu \mathrm{IDTT} 0.1 \mathrm{M}$.

Mowiol: $4.8 \mathrm{~g} \mathrm{MOWIOL}{ }^{\circledR} 4-88$ in $12 \mathrm{~g}$ glycerol and mix. Add $6 \mathrm{ml}$ of water and stir for several hours at RT. Add $12 \mathrm{ml}$ of $0.2 \mathrm{M}$ Tris $(\mathrm{pH} 8.5)$ and heat to $50^{\circ} \mathrm{C}$ for $10 \mathrm{~min}$ with occasional mixing. After the MOWIOL ${ }^{\circledR}$ 4-88 dissolves, clarify the solution by centrifugation at $5000 \times \mathrm{g}$ for $15 \mathrm{~min}$. After reconstitution mowiol was aliquoted and frozen $\left(-20^{\circ} \mathrm{C}\right)$ for long-term storage or kept at $4^{\circ} \mathrm{C}$ for short-term storage. 
PBS: $9.55 \mathrm{~g} / \mathrm{l} \mathrm{PBS}$ in bi-distilled water $\rightarrow$ filter and autoclave.

Releasing Buffer (RB): $135 \mathrm{mM} \mathrm{NaCl}, 1 \mathrm{mM} \mathrm{MgCl}$, 1,2 mM CaCl, 2 mM NaH${ }_{2} \mathrm{PO}_{4}, 10 \mathrm{mM}$ glucose, 56 $\mathrm{mM} \mathrm{KCl}$. Filtered.

Resolving gel: $2000 \mu \mathrm{l}$ of $30 \%$ acrylamide were mixed with $1250 \mu$ l of Tris $\mathrm{pH} 8.8,1750 \mu \mathrm{l}$ of $\mathrm{H}_{2} \mathrm{O}, 15$ $\mu \mathrm{l}$ of $10 \%$ APS and $3 \mu \mathrm{l}$ of TEMED.

Saline solution: $9 \mathrm{~g} \mathrm{NaCl}$ in bi-distilled water $\rightarrow$ filter and autoclave.

SDS running buffer (10X 1 I): $30.24 \mathrm{~g}$ Tris, $142.5 \mathrm{~g}$ glycin, $10 \mathrm{~g}$ SDS $\rightarrow$ filter and autoclave.

Soc++ media: $2 \%$ bacto-tryptone, $0.5 \%$ yeast extract, $10 \mathrm{mM} \mathrm{NaCl}, 2.5 \mathrm{mM} \mathrm{KCl}, 10 \mathrm{mM} \mathrm{MgCl}, 10$ $\mathrm{mM}$ MgSO4, $20 \mathrm{mM}$ glucose $\mathrm{pH}$ 7.0) Add Glucose after autoclaving the solution with the remaining ingredients.

Stacking gel: $217 \mu \mathrm{l}$ of $30 \%$ acrylamide, $417 \mu \mathrm{l}$ Tris $\mathrm{pH} 6.8,1017 \mu \mathrm{l}$ of $\mathrm{H}_{2} \mathrm{O}, 8.33 \mu \mathrm{l} 10 \%$ APS and $1.7 \mu \mathrm{l}$ TEMED.

TBE buffer (10X $1 \mathrm{l})$ : $108 \mathrm{~g}$ Tris, 55g boric acid, $9.3 \mathrm{~g}$ EDTA, bi-distilled water to $1 \mathrm{I} \rightarrow$ filter and autoclave.

TBS: $150 \mathrm{mM} \mathrm{NaCl}, 10 \mathrm{mM}$ Tris-HCl, pH 9.0 (for antigen retrieval).

TBS-T: 150 mM NaCl, 10 mM Tris-HCl, 0.1\% Tween 20, pH 7.6.

TE buffer: $10 \mathrm{mM}$ Tris-HCl, 1mM EDTA, pH 8.0.

Transfer buffer: 192 mM Glycine, 20\% Methanol, 25 mM Tris-HCl, pH 8.3.

TRIS: $10 \mathrm{mM}$ tris-buffered saline $\mathrm{pH}$ 8.0.

\subsection{Enzymes}

DNA polymerase I large klenow fragment + Klenow buffer: New England Biolabs (NEB)

DNAse I + buffer: New England Biolabs (NEB)

Phusion high fidelity DNA polymerase + buffer: Finnzymes

Proteinase K + buffer: New England Biolabs (NEB)

Restriction enzymes + buffers + BSA: New England Biolabs (NEB)

Shrimps Alkaline Phosphatase + dephosphorilation buffer: Roche

T4 DNA ligase + ligation buffer: New England Biolabs (NEB)

\subsection{Kits}

BCA $^{\text {TM }}$ protein assay kit: Thermo scientific

DNA gel extraction kit: Quiagen

GDNF $E_{\text {MAX }}{ }^{\circledR}$ ImmunoAssay System: Promega 
Miniprep kit: Quiagen

Midiprep kit: Macherei Nagel

Megaprep kit: Macherei Nagel

PCR purification kit: Quiagen

Platinum SYRB ${ }^{\circledR}$ Green qPCR SuperMix-UDG: Invitrogen

pSwitch ${ }^{\text {TM }}$ system: Invitrogen

2.5 PCR primers for sequencing and cloning

\begin{tabular}{|l|l|}
\hline \multicolumn{1}{|c|}{ Name } & \multicolumn{1}{c|}{ Sequence } \\
\hline AAAD forward & AGTAGATCTGCTAGCCCACCATGAACGCAAGTGAATTCCG \\
\hline AAAD reverse & GATATCGATAAGCTTCTACTCCCTCTCTGCTCGCAGCACG \\
\hline BGHpA forward & CTAGTTGCCAGCCATCTGTT \\
\hline BGHpA reverse & CTACTCAGACAATGCGATGC \\
\hline BGHpA reverse 2 & CCCCAGAATAGAATGACACC \\
\hline EGFP reverse & ACTTCAGGGTCAGCTTGCCGTA \\
\hline IVS8 forward & TTCTCTTCACAGGCCACCAA \\
\hline IRES forward & CGTGGCGGAACCGACTACTT \\
\hline IRES reverse & ATTAGCGGCCGCCCTGCAGGTCAGTCACTTTCAGATTCTTCATCTTC \\
\hline pSwitch forward & CTTGGATGAGCTTAATGGTG \\
\hline pSwitch forward 2 & GCTATAACTCGCCTAGTGAC \\
\hline pSwitch reverse & GCCTTGATTCCACTTCTGTC \\
\hline TB forward & TAAGGTACGGGAGGTACTTGGA \\
\hline TH forward & ATTAGCGGCCGCGAATTCCCACCATGCCCACCCCCAGCGCCT \\
\hline TH reverse & CTGTAAGCTTCCTGCAGGTTAGCTAATGGCACTCAGTGCTTGGG \\
\hline UAS TATA forward & AAAATAGATCTACGCGTAAGGATCCGGGCCCAAGCGGGTACTGTCCTCCGAGTGGA \\
\hline UAS TATA reverse & AATATAGATCTATGCATCCGCATGCGTGAAGCTTACTAGTGTACCGGTAGCTCGGTAC \\
\hline Syn forward & GAGGGCTAGCTTGGTGGCCTGTGAAG \\
\hline VMAT2 forward & ATTAGGATCCGAATTCCCACCATGGCCCTGAGCGAGCTGGCGCT \\
\hline VMAT2 reverse & ATTAGCGGCCGCCCTGCAGGTCAGTCACTTTCAGATTCTTCATCTTC \\
\hline
\end{tabular}

Table 2) list of primer used for sequencing and cloning. AAAD = aromatic amino avid decarboxylase; $B G H p A=$ bovine growth factor polyadenylation sequence; EGFP = enhanced green fluorescence protein; IVS8 = synthetic intron; $p S w i t c h=$ pSwitch protein; TB = transcription blocker; $\mathrm{TH}$ = tyrosine hydroxylase; syn = synapsin promoter; VMAT2 = Vesicular monoamine transporter 2.

\subsection{Oligonucleotides}

\begin{tabular}{|c|l|}
\hline Name & \multicolumn{1}{|c|}{ Sequence } \\
\hline Oligo A & Blunt-GGGCCCAACCTGCAGGATATGGATCCATATTCCGGAATAGTCGAC-Blunt \\
\hline Oligo B & Blunt-GGGCCCACCTGATATCATCTGAATTCATAGGGATCC-Blunt \\
\hline Oligo C & $\begin{array}{l}\text { Bgl2-- } \\
\text { ATCAATGCATGATATCACGCGTAGGCTAGCTCCCTGCAGGAGGATCCGTTAATTAAGCACCGGTCT } \\
\text { CAGATCTGCGGCCGCAATGAGCTCTTGCATGCT- BgI2- }\end{array}$ \\
\hline Oligo D & Age1 - CCGGTATCCTGCAGGTTATCGATGATAGCGGCCGCATTCGCTAGCTGATAAGCTT - Cla1 \\
\hline
\end{tabular}

Table 3) Double strand DNAs for clonation. Oligo = oligonucleotide 


\subsection{Plasmids}

pAAV SEWB: kindly provided by Dr. Sebastian Kügler, University Hospital Göttingen, Göttingen, Germany

pAAV syn-GDNF-WB: kindly provided by Dr. Sebastian Kügler, University Hospital Göttingen, Göttingen, Germany

pGEM4Z4: Stratagene

ptyf 2xGfABC1D: Kindly provided byMichael Brenner University of Alabama at Birmingham, Birmingham, Alabama [101]

6p 3TBXright: kindly provided by Dr. Sebastian Kügler, University Hospital Göttingen, Göttingen, Germany

PTR-UF22: UOP Vector Core Florida

pAAV HBADsREd: kindly provided by Dr. Sebastian Kügler, University Hospital Göttingen, Göttingen, Germany

pDP5: Plasmid factory Heidelberg

pDP6: Plasmid factory Heidelberg

\subsection{Cells and animals}

DH5 $\alpha$ E. Coli strain: ElectroMAXTMDH5 $\alpha$-ETMCells, Invitrogen

Sure E. Coli strain: SURE ${ }^{\circledR}$ Electroporation-Competent Cells (Stop Unwanted Rearrangement Events); Stratagene

HEK 293: Stratagene

Wistar rats: Charles Rlver

NMRI and C57/BI6 mice: Zentrale Tierexperimentelle Einrichtung (ZTE) Universitätmedizin Göttingen 


\section{Methods}

\subsection{Molecular biology}

\subsubsection{Oligo annealing}

After being designed using SECentral (Scientific \& Educational Software, NC, US) software, oligonucelotides with proper sticky ends and restriction sites were purchased from SigmaAldricht as single DNA sequences.

The lyophilized oligos were resuspended in $\mathrm{H}_{2} \mathrm{O}$ at a concentration of $200 \mu \mathrm{M} ; 5 \mu$ of each oligonucleotide were diluted with $2 \mu \mathrm{l}$ of $10 \mathrm{X}$ annealing buffer and $8 \mu \mathrm{l}$ of $\mathrm{H}_{2} \mathrm{O}$; the mixture was heated at $95^{\circ} \mathrm{C}$ for $4 \mathrm{~min}$ and the annealing was promoted by cooling down the solution for $5-10$ minutes at room temperature. The solution was then spun down, diluted at 1.10 .000 and used for subsequent ligations.

\subsubsection{PCR amplification}

Primers for PCR amplification were designed using SECentral such that a minimum of 20 bp was complementary to the target sequences and appropriate overhangs, with desired restriction sites were added.

PCR amplification conditions differed for each pair of plasmid/primers. Different conditions were tested in order to optimize the combination of $\mathrm{MgCl}_{2}$ concentration $(0,0.5,1 \mathrm{mM})$ and annealing temperature (ranging from 60 to $72^{\circ} \mathrm{C}$ ).

PCR reaction mix contained: Phusion HF (high fidelity) reaction buffer, $0-1 \mathrm{mM} \mathrm{MgCl} 2,500 \mathrm{nM}$ of each sense and antisense primers, 200 $\mu \mathrm{M}$ of each dATP, dGTP, dCTP, dTTP, 5-10ng of the template DNA, 0.6 unit of the Phusion High-Fidelity DNA polymerase, and Ampuwa water to a final volume of $50 \mu \mathrm{H}$. After $1 \mathrm{~min}$ incubation at $98^{\circ} \mathrm{C}$ for denaturation the reaction was subjected to 35 cycles of amplification each of them was formed by $15 \mathrm{sec}$ at $98^{\circ} \mathrm{C}$ for denaturation, $15 \mathrm{sec}$ at $60-72^{\circ} \mathrm{C}$ for annealing the primers and $20 \mathrm{sec} / \mathrm{kb}$ at $72^{\circ} \mathrm{C}$ for the elongation; a final step of $10 \mathrm{~min}$ at $72^{\circ} \mathrm{C}$ was performed to allow a final elongation.

One or two $\mu$ l of the DNA products were then tested by gel electrophoresis and the rest was purified with the PCR purification kit (described later).

\subsubsection{Restriction enzymes}

DNA restriction enzymes used in molecular biology are nucleases that recognize specific sequences of DNA and cut them in a specific way. As their activity is predictable, we used them to generate DNA fragments with specific overhangs that can be used for ligation and to cut the DNA and verify if the resulted fragments are compatible with what we expected by molecular cut in silicio (control digestion).

DNA restriction reactions were carried out following the manufacturer's instructions. If the digestion product was to be used for cloning a total of 4-5 $\mu \mathrm{g}$ of DNA was used. For control digestions, a total of $100 \mathrm{ng}$ of DNA was digested. The DNA was mixed with the appropriate 10X buffer, 10X BSA if necessary, $Y$ unit of restriction enzyme $\left(Y=X \mu g\right.$ of $\left.D N A^{*} 6\right)$ and the volume was brought to $50 \mu$ l (in case of cloning) or $25 \mu \mathrm{l}$ (in case of control digestion) with Ampuwa water. The reaction was incubated for $60 \mathrm{~min}$ at the appropriate temperature and the reaction was stopped by putting the 
samples on ice. When the DNA had to be cut with two different but compatible enzymes regarding the buffer and the reaction temperature they were used together in a double reaction mix.

\subsubsection{Gel electrophoresis}

Agarose gel electrophoresis was performed for different reasons: a) to separate different fragments of DNA after the restriction reaction; $b$ ) to check qualitatively the DNA after a control digestion or a PCR reaction; and c) to quantify the amount of DNA obtained after gel extraction or PCR purification.

a) To separate DNA fragments for subsequent manipulations the $50 \mu$ restriction reaction was diluted with Ampuwa water and $6 \mathrm{X}$ loading buffer to a volume of $300 \mu \mathrm{l}$; the obtained mixture was then loaded in 6 different wells.

b) To check the sizes of the DNA's fragments after a control digestion $5 \mu l$ of loading buffer were added to the $25 \mu \mathrm{l}$ reaction tube and the whole volume was loaded in a single well. If a PCR reaction had to be checked, 1-2 $\mu$ l of the reaction were diluted with $20 \mu \mathrm{l}$ of Ampuwa water and $5 \mu$ of $6 \mathrm{X}$ loading buffer; the mixture was then loaded in a single well.

c) For DNA quantification 1 and $2 \mu$ of DNA solution was diluted with Ampuwa water and 6X loading buffer till a final volume of $20 \mu$; these mixtures were then loaded in the gel together with different $(50,100,200,400$ ng total DNA) known amount of HIND III Lambda DNA; by comparing the intensity under a UV light of the know samples is possible to estimate the concentration of the un-know samples (figure 3).

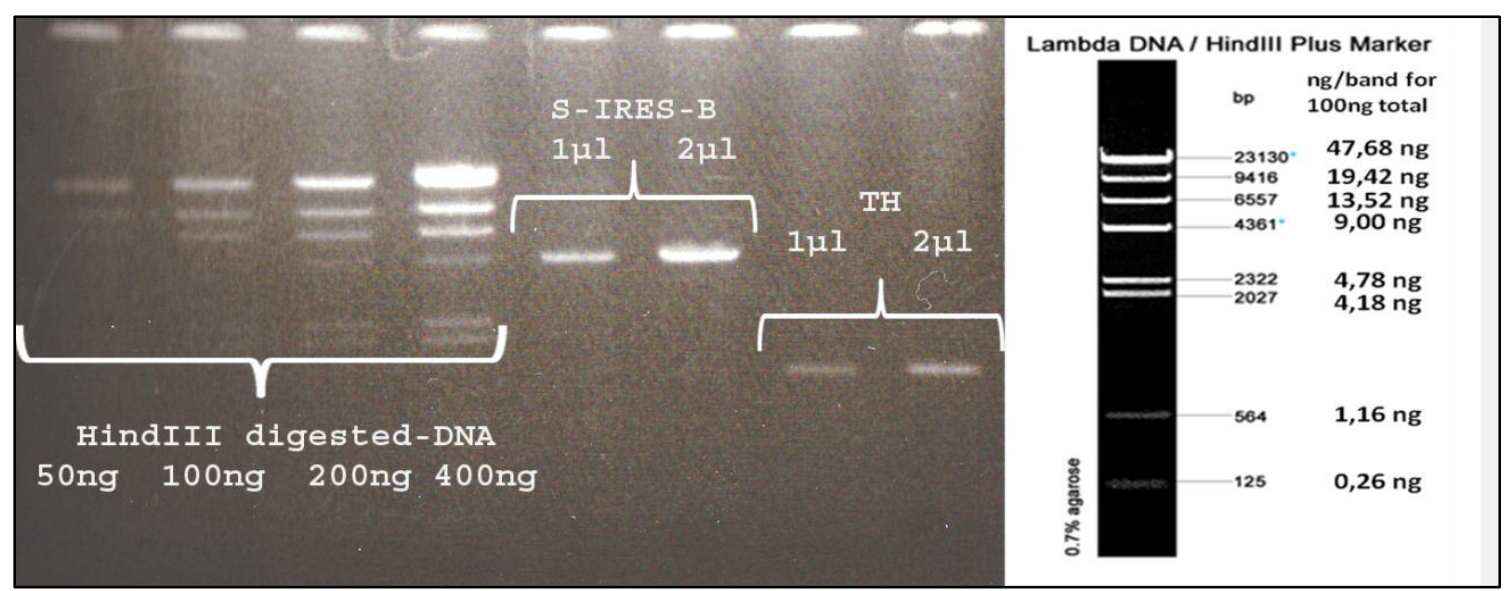

Figure 3) Gel quantification for AAV S-IRES-B and TH, compared to 50, 100, 200 and $400 \mathrm{ng}$ of HindIII-digested $\lambda$ DNA. Comparing the relative amount of each band with the standard (right panel) it is possible to evaluate the amounts of the un-known samples (AAV S-IRES-B and TH). In this case concentration of AAV S-IRES-BG was decided to be $\sim 75 \mathrm{ng} / \mu \mathrm{l}$ and of $\mathrm{TH} \sim 24 \mathrm{ng} / \mu \mathrm{l}$

In all cases a 2-Log DNA marker (a mixture of DNA fragments with defined sizes) was loaded in the gels in order to verify the sizes of the bands.

For gel preparation different quantities of agar, depending on the size of the target fragments ranging from $0.6 \%(>3000 \mathrm{bp}$ ) to $2 \%(<400 \mathrm{bp})$, were dissolved in TBE buffer by heating the mixture in a microwave. Once dissolved, the agar was cooled down to about $40^{\circ} \mathrm{C}$ and $1 \mu$ l of ethidium bromide per $50 \mathrm{ml}$ of solution was added. The mixture was then poured to a gel tray with a proper comb and left to polymerize at room temperature. Gels were then placed in a electrophoresis chamber and immersed in TBE buffer; samples and marker were loaded and run for 40-60 min at 70-100 V. DNA bands were visualized by UV-light in a Gel Documentation 2000 UV-transilluminator coupled with a computer with the Quantity One software (BioRad). 


\subsubsection{DNA gel extraction kit (GE)}

To isolate different fragments of DNA after a restriction reaction the mixture was run in agarose gel; the DNA band with the corresponding size of what was predicted in silicio was physically cut from the gel with a knife and the DNA was purified with the Quiagen gel extraction kit (Quiagen).

The procedure was performed following the manufacturer's instructions. Briefly, the gel piece was weighted and dissolved in dissolving buffer at $50^{\circ} \mathrm{C}$ for $10 \mathrm{~min}$; the obtained solution was then loaded to an ion exchange column in high salt condition which allows the binding of the DNA to the silica column; after a washing step, the DNA was eluted in low salt conditions with $30 \mu$ l elution buffer or Ampuwa water. The purity and concentration of the obtained DNA was then evaluated by gel electrophoresis.

\subsubsection{PCR purification kit (PP)}

To isolate a linearized DNA or to purify a DNA fragment from oligonucleotides smaller than 40bp from a PCR reaction a Quiagen PCR purification kit was used.

This was performed following the manufacture's instruction: briefly, the DNA solution was diluted in loading buffer, load to an ion exchange column in high salt condition, washed, and eluted in low salt condition with $30 \mu \mathrm{l}$ of elution buffer or Ampuwa water.

\subsubsection{Remove or fill in DNA overhangs to create blunt ends}

When it was not possible to obtain compatible sticky ends, the DNA fragments were treated in order to create blunt ends using the DNA polymerase I large klenow fragment an enzyme used to remove the 5 'overhangs and to fill the 3 'overhangs. The resulting DNA was incubated with $1.3 \mu \mathrm{l}$ of the enzyme ( $5 \mathrm{U} / \mu \mathrm{l}$, total of $6.5 \mathrm{Units}$ ), $0.9 \mu \mathrm{l}$ of $2 \mathrm{mM}$ dNTPS (final concentration $0.33 \mu \mathrm{M}$ ), 10X klenow buffer and the volume was brought to $50 \mu \mathrm{l}$ final volume with Ampuwa water. The reaction was incubated for $15 \mathrm{~min}$ at $25^{\circ} \mathrm{C}$ and the DNA was then purified with the PCR purification kit.

\subsubsection{Dephosphorilation}

To avoid uni-molecular ligation in case of blunt ends or compatible sticky ends, a dephosphorilation of the 5 'end of the backbone is necessary.

The dephosphorilation was performed by mixing 200 ng of DNA with $4 \mu \mathrm{l}$ X dephosphorilation buffer, $1.5 \mu$ of shrimp Alkaline phosphatase (1 unit/ $\mu$ l) and Ampuwa water in a final volume of 20 $\mu \mathrm{l}$. The mixture was incubated for $25 \mathrm{~min}$ at $37^{\circ} \mathrm{C}$ and the enzyme was then inactivated by an additional incubation of $15 \mathrm{~min}$ at $65^{\circ} \mathrm{C}$. The DNA was then directly used for ligation.

\subsubsection{Ligation}

The ligation reaction consists in the enzymatic joining of two linearized fragments of DNA with compatible ends.

A total amount of $200 \mathrm{ng}$ of DNA with a ratio of 1:3 (weight/weight) between the backbone and the insert was incubated with $5 \mathrm{X}$ ligation buffer and $1 \mu \mathrm{l}$ (400 units) of T4 DNA ligase. The volume was then brought to 20 or $30 \mu \mathrm{l}$ with Ampuwa water. The mixture was then incubated at room temperature for $20 \mathrm{~min}$ in case the fragments were with sticky ends and for 1 hour in case of blunt ends. The reaction was then stopped by putting the mixture on ice and used directly for bacteria transformation. A negative control was performed by omitting the addition of the insert into the mixture. 


\subsubsection{E.Coli transformation, seeding and expansion}

In order to produce a large quantity of the cloned plasmid after ligation, the obtained DNA was used to transform E. Coli bacteria which were then selected by antibiotic resistance present in the backbone of the vectors (the antibiotic resistance will be present only in transformed bacteria) and expanded. For normal vectors DH5 $\alpha$ E.Coli were used while for AAV vectors Sure E.Coli bacteria were used.

One to $4 \mu \mathrm{l}$ of the ligation reaction were added to $\sim 70 \mu$ l of fresh thawed electrocompetent bacteria and kept on ice; the mixture was transferred to a pre-cooled electroporation cuvette which was then subjected to a electroshock pulse procedure at Bio-rad Gene Pulser II (Voltage $=1.8 \mathrm{kV}$, pulse controller- low resistance $=200 \mathrm{Ohm}$, capacitance $=25 \mu \mathrm{F}$ ). The mixture was then transferred to a 2 $\mathrm{ml}$ eppendorf tube together with $1 \mathrm{ml}$ of pre-warmed SOC++ media, and incubated in a shaker at 200 $\mathrm{rpm}$ and $37^{\circ} \mathrm{C}$ for $45 \mathrm{~min}$. This step allows the bacteria to recover after the electroshock and to express the antibiotic resistance. Sixty to $500 \mu \mathrm{l}$ of bacteria were then seeded on LB agar plate with the appropriate antibiotic; plates were left at $37^{\circ} \mathrm{C}$ over-night to allow the selected clones to grow.

LB agar plates were prepared by dissolving $40 \mathrm{~g} / \mathrm{I}$ of LB-Agar (Applichem) in bi-distilled water. The mixture was then autoclaved and cooled down to $50-60^{\circ} \mathrm{C}$. Antibiotics were then added to the solution (ampicillin $=100 \mathrm{mg} / \mathrm{l}$, kanamicin $=50 \mathrm{mg} / \mathrm{l}$ ) and $20-25 \mathrm{ml} /$ plate were poured in $10 \mathrm{~cm}$ plates and let to polymerize. Plates were then stored at $4^{\circ} \mathrm{C}$.

Singles colonies were then repicked and transferred in LB media supplemented with antibiotic (100 $\mu \mathrm{g} / \mathrm{ml}$ ampicillin or $50 \mu \mathrm{g} / \mathrm{ml}$ kanamicin). To prepare the LB media $25 \mathrm{~g} / \mathrm{l}$ of LB were dissolved in bidistilled water and autoclaved. In case of mini-prep $5 \mathrm{ml}$ of media were used and the cultures were left over night at $37^{\circ} \mathrm{C}$ under $200 \mathrm{rmp}$ shaking. In case of Midi- or Mega-prep a pre-culture was done by incubating a single colony for $\sim 6-8$ hours in 2-3 $\mathrm{ml}$ of LB media (supplemented with antibiotics) under shaking at $37^{\circ} \mathrm{C}$. The main culture was started by transferring bacteria to fresh LB media at 1:1000 ratio; total volume for a Midi-prep was $100 \mathrm{ml}$ while for a Mega-prep the final volume was $2000 \mathrm{ml}$. Cultures were left o/n at $37^{\circ} \mathrm{C}$ with $1000 \mathrm{rpm}$ shaking.

\subsubsection{Production of electro-competent E.Coli cells}

Electrocompetent cells (DH5 $\alpha$ and SURE cells) from glycerol stock were plated in a LB agar plate without antibiotics and left overnight to grow; the following day a single colony was repicked, transferred to $10 \mathrm{ml} \mathrm{LB}$ media and incubated overnight at $37^{\circ} \mathrm{C}$ under shaking. The following day 2 liters of pre-warmed LB media were inoculated with 3-4 $\mathrm{ml}$ of the culture and the bacteria were left to expand at $37^{\circ} \mathrm{C}$ under $100 \mathrm{rpm}$ shaking till they reached an OD between 0.8 and 1.0 (pure LB media was used as a reference; the procedure takes 3-5 hours). Bacteria were then transferred in 6 ultracentrifuge tubes ( $350 \mathrm{ml}$ each) and put on ice for one hour. Next the suspended bacteria were centrifuged at $\sim 2700 \mathrm{~g}$ for $10 \mathrm{~min}$, the supernatant discarded and the bacteria pellet was resuspended in 2-3 liters of $4^{\circ} \mathrm{C}$ sterile water. The centrifugation and resuspension was repeated another two times, transferring the bacteria first to three ultracentrifugation tubes with sterile water and then to one $50 \mathrm{ml}$ falcon tube with $10 \%$ glycerol in water. Cells were centrifuged again and the final resuspension was done by adding a volume of water $10 \%$ glycerol equal to the volume of the pellet. Bacteria were then aliquoted in $70-80 \mu \mathrm{l}$ aliquots in pre-cooled $0.5 \mathrm{ml}$ tubes and stored at $80^{\circ} \mathrm{C}$. 


\subsubsection{Mini-, midi-, mega-prep}

To extract the plasmid DNA from bacteria after expansion 3 kits were used depending on the size of the preparation; Mini-prep (Quiagen) for $5 \mathrm{ml}$ bacteria, Midi-prep (Macherey-Nagel) for $100 \mathrm{ml}$ bacteria, and Mega-prep (Macherey-Nagel) for $2000 \mathrm{ml}$ bacteria. In case of a new construct $0.5 \mathrm{ml}$ of bacteria were mixed with $0.5 \mathrm{ml}$ of freezing solution and kept at $-80^{\circ} \mathrm{C}$ as a bacteria glycerol stock.

The preparations were done following the instructions of the manufactures; the principle of the preparations is always the same with only slight differences in some buffers composition.

After overnight expansion bacteria are precipitated with a $6000 \mathrm{~g}$ force and resuspended in resuspension buffer supplemented with RNAse to degrade RNA. The bacteria are then subject to an alkaline lysis in which the plasmid DNA stays in solution while the debris formed by membranes and protein is precipitated by centrifugation at $16000 \mathrm{~g}$; the supernatant is then loaded to a ion exchange column in a high salt condition that allow the plasmid DNA to bind to the resin. In the case of Midi and Mega-prep the column has to be pre-equilibrated before its use. After washing the column, the plasmid DNA is eluted in low salt condition with water or elution buffer. In the case of Midi and Mega prep the total volume of bacteria was divided by 2 and the same column was used twice previous reequilibration.

To further purify the DNA in the case of Midi- and Mega-prep the eluted plasmid was precipitated with isoprophanol, washed with $70 \%$ ethanol, dried at room temperature and resuspended over night in TE buffer. DNA concentration and purity was checked using a biophotometer which can calculate the DNA concentration using the Lambert-Beer law $A=\varepsilon b c$, in which $A=$ absorbance, $\varepsilon=$ molar absorbitivity, $\mathrm{b}=$ chamber length in $\mathrm{cm}$, and $\mathrm{c}=$ concentration; by measuring the absorbance at $260 \mathrm{~nm}$ (the wavelength that is absorbed by aromatic groups) and knowing $\varepsilon$ and $b$ the DNA concentration can be calculated; moreover the machine measures the absorbance at $280 \mathrm{~nm}$ (absorbance of tryptophan) and by calculating the ratio $260 \mathrm{~nm} / 280 \mathrm{~nm}$ the DNA purity from protein can be estimated; a ratio over 1.80 was considered acceptable.

\subsubsection{Quality control of the plasmid}

To verify that all the cloning reactions led to the desired plasmids different control digestion followed by gel electrophoresis were performed. To predict the expected DNA fragments the control digestion was first simulated in silicio using SECentral and then was performed practically. Single or double digestions were chosen in order to obtain fragments that could be easily discriminate in a gel electrophoresis.

To verify the integrity of the two ITRs all AAV plasmids were digested with Sma1; the enzyme recognizes 2 sequences in the two ITRs so its use leads a typical pattern in the gel electrophoresis with a DNA band at $3013 \mathrm{bp}$ which corresponds to the backbone of the AAV vector.

In case of any doubts or when new DNA sequences were purchased from different companies the material was sent to the Seqlab (Sequence laboratories Göttingen $\mathrm{GmbH}$ ) for an "extended Hot Shot sequencing reaction"; 600-700 ng of plasmid DNA and 20 pmol of primer were mixed in a $0.2 \mathrm{ml}$ flatlid vial and the volume was brought to $7 \mu$ using $10 \mathrm{mM}$ Tris pH 8.0. Primers were chosen depending on the vector and they were designed in order to pair to a know sequence and be able to elongate in the direction of the unknown sequence (table 2). 


\subsection{Vector cloning}

All cloning steps and oligonucleotides were first virtually designed using SECentral software.

The pSwitch ${ }^{T M}$ system was purchased from invitrogen ${ }^{T M}$; the kit includes a vector encoding the pSwitch protein and 3 responsive vector pGene A, B, C with 3 different open reading frames.

All cloned vectors were checked by control digestion and by sequencing.

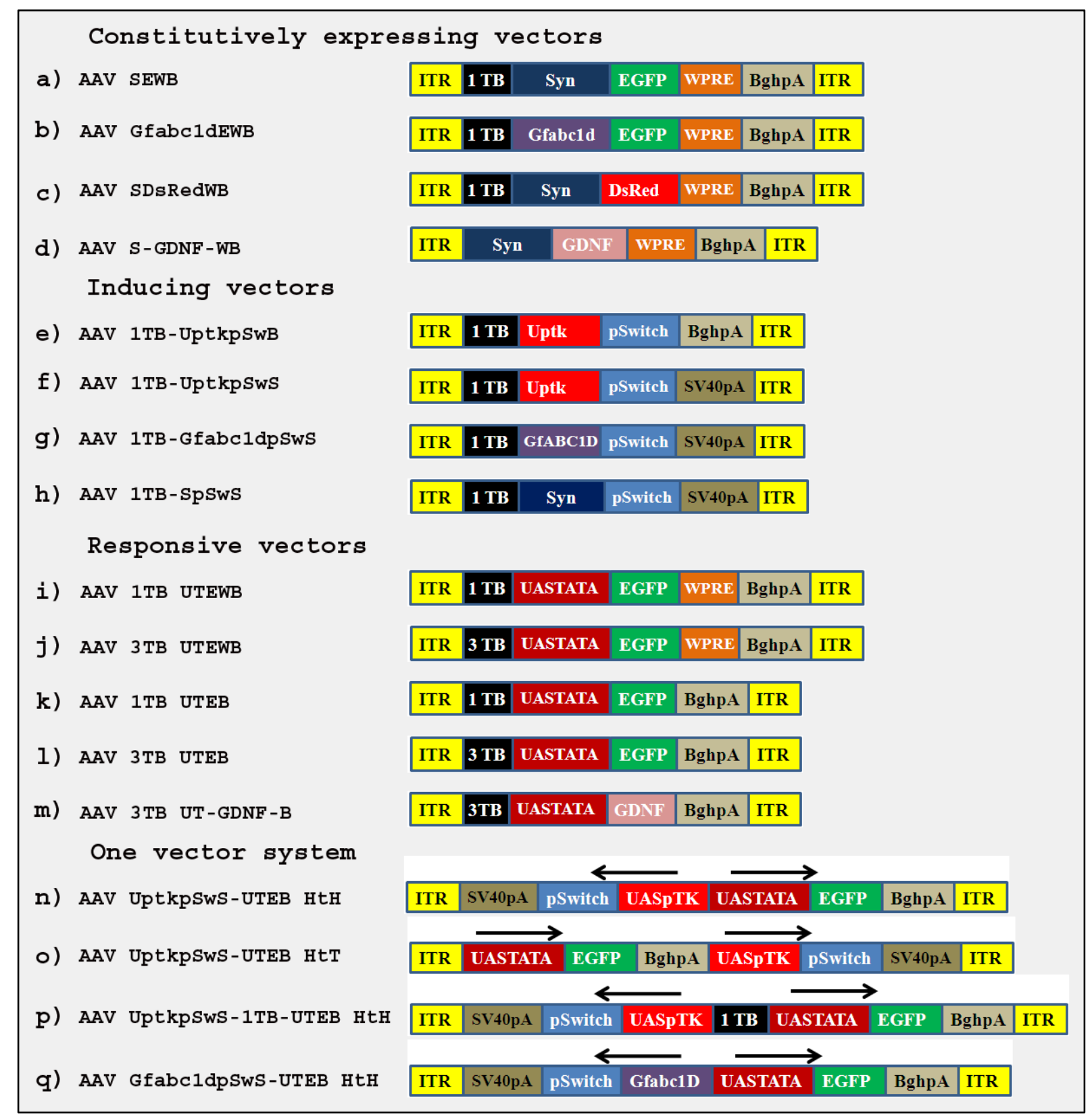

Figure 4) Representation of the main used plasmids: $A A V=$ adeno associated virus; ITR = inverted terminal repeat; TB = transcription blocker; Syn = S = synapsin promoter; EGFP = enhanced green fluorescence protein; WPRE = Woodchuck Hepatitis Virus (WHP) Posttranscriptional Regulatory Element; Gfabc1d = astrocytic promoter; BghpA = B = bovine growth hormone poli adenilation sequence; DsRed = red fluorescent protein; GDNF = glial cell derived neurotrophic factor; Uptk = Uptk = UAS (Upstream activatin sequence) Herpes Simplex Virus thymidine kinase (TK) minimal promoter; $\mathrm{pSwitch}=\mathrm{pSw}=\mathrm{pS}$ witch protein; SV40pA = S = Simian virus 40 poli adenilation sequence; $\mathrm{HtH}=$ head to head; $\mathrm{HtT}=\mathrm{head}$ to tail. 


\subsubsection{AAV vector genomes expressing the pSwitch protein}

The first cloning step consisted in transferring the DNA sequence encoding for the pSwitch protein and its inducible promoter to an AAV vector. Oligonucleotide A (table 3 ) was designed with overhangs compatible with Apa1 and Sal1 restriction sites. AAV SEWB (figure 4-a) was digested with Apa1 and Sal1, purified by GE, and oligo A was inserted resulting in AAV-OligoA-B. This and the original pSwitch vector were then cut with Sbf1 and BamH1 and the UAS-ptk-pSw fragment, isolated by GE, was inserted in AAV-OligoA-B backbone, purified by PP, resulting in AAV-UptkpSw-B (figure 4e).

For further cloning and easier manipulation of the pSwitch cassette another oligonucleotide, with mutated Bgl2 overhangs, was designed (Oligo C; table 3). The mutated overhang results in an oligonucleotide that can be ligated but cannot be re-cut in the same site. AAV SEWB was cut with $\mathrm{Bg} \mid 2$ and the OligoC was inserted in the AAV backbone, purified by GE, resulting in AAV-OligoC. To insert the SV40 poli-adenylation sequence both the AAV-OligoC and the vector containing the SV40 sequence, pGEM4Z4, were cut with Not1 and Sac1 and the DNA corresponding to the SV40 sequence was isolated by GE and cloned in the AAV backbone, purified by PP, resulting in AAV-OligoC SV40 vector. In the following step the DNA sequence for the pSwitch protein was cut from AAV-ptkpSw-B with BamH1 and Age1and isolated by GE. The AAV-OligoC-SV40 was cut with Bgl2 and Age1 and then purified by PP. As Bgl2 and BamH1 give compatible overhangs it was possible to ligate the two fragments obtaining the AAV-OligoC-pSw-S vector.

In order to add the ptk promoter to AAV-OligoC-pSw-SV40 this last vector and AAV-ptkpSw-B were cut with Sbf1 and Pac1 and the fragment corresponding to the promoter was isolated by GE and cloned into the backbone. The ligation product was named AAV-Uptk-pSw-S (figure 4-f).

To restrict the expression of the pSwitch protein to astrocytes the astrocytic promoter Gfabc1d was inserted in the vector AAV-OligoC-pSw-S. Both the vectors ptyf $2 x G f a b c 1 d$, which contains the desired promoter, and AAV-OligoC-pSw-S were cut with Mlu1 and BamH1 and the fragment corresponding to the promoter was inserted in the backbone obtaining AAV-GfABC1D-pSw-S (figure 4-g); both fragments were purified by GE.

In another construct, instead of the astrocytic promoter, a neuronal specific synapsin promoter was used. The synapsin promoter was cut from AAV SEWB using BamH1 and Mlu1 and isolated by GE. Using the same enzymes as above, the astrocytic promoter was removed from AAV-GfaABC1D-pSw-S and the BB was purified by GE. The synapsin promoter was then inserted in the new backbone in order to obtain AAV SpSwS (figure 4-h).

\subsubsection{AAV vector genome with responsive promoter}

To facilitate the cloning of the responsive vectors the oligonucleotide Oligo-B (table 3 ) was designed and purchased from Sigma. Oligo-B and AAV-SEWB were cut with Apa1 and BamH1 and, after GE, the oligonucleotide was cloned in the backbone. The resulting AAV-OligoB-EWB was cut using EcoRV and BamHI and the backbone was isolated by PP while the UAS-TATA from the original responsive vector was cut with Ssp1 and BamH1 and isolated by GE. As both EcoRV and Sspl give blunt ends, the ligation between the AAV vector and the UAS-TATA fragment was possible and a vector named AAV 1TB UTEWB (figure 4-i) was obtained.

To excise the WPRE (WHP Post-trascriptional Response Element), and decrease the expression of EGFP AAV-OligoB-EWB was cut with Hind3 and Hinc2. The 2 enzymes were then inactivated, the 
desired DNA vector was separated from the WPRE sequence by GE, and a reaction with the Klenow fragment was performed to obtain blunt ends. The vector was then purified by PP and re-circularized to obtain AAV-OligoB-EB. This last vector was then cut with EcoRV and BamH1, purified by PP, and ligated with the UAS promoter obtained by cutting the original responsive vector with Ssp1 and Bamh1. The obtained vector was named AAV-1TB-UTEB (figure 4-k).

For an easier manipulation of the responsive cassette two primers designed to target and amplify the UAS-TATA sequence from AAV 1TB UTEB (UAS TATA forward and reverse; table 2) with appropriate overhangs were purchased from Sigma. As there was the need to eliminate a Hind3 restriction site in the target sequence, AAV-1TB-UTEB was cut with Hind3 and the resulted linearized DNA was treated with the Klenow enzyme; the DNA was then purified by PP, and ligated to re-circulize it and obtain AAV-1TB-UTEB Hind3'. The two primers were used on this vector with annealing temperature of $68^{\circ} \mathrm{C}$ and $1 \mu \mathrm{M} \mathrm{MgCl}_{2}$ to obtain the Oligo-UASTATA sequence that was then cut with $\mathrm{Bg} / 2$ and purified by PP. AAV SEWB was cut with Bgl2 and dephosphorilated. The obtained backbone, isolated by GE, was ligated with the Oligo-UASTATA to attain AAV-Oligo-UASTATA. To add the EGFP and the WPRE this last vector and AAV SEWB were cut with Hind3 and Sph1 and the fragment corresponding to EGFP-WPRE, isolated by GE, was add to the backbone and isolated by PP, to obtain AAV UTEWB.

To isolate the ITR effect on the promoter 3 transcription blockers (TB) were added. AAV UTEWB and AAV 6p 3TBxright were cut with Mlu1 and BamH1. The backbone from AAV-UTEWB, purified by PP, and the fragment corresponding to the $3 \mathrm{~TB}$, isolated by GE, were ligated and the resulted vector was named AAV-3TB-UTEWB (figure 4-j).

To remove the WPRE sequence AAV UTEWB was cut with Cla1 and after a GE the fragment corresponding to the open vector was re-circulized; the resulted vector was named AAV-UTEB.

Like in the previous vector to isolate the ITR effect on the promoter 3TB were added; AAV $6 p$ 3TBxright and AAV-UTEB were cut with Mlu1 and BamH1 and the 3TB fragment was inserted to the backbone to obtain AAV 3TB-UTEB (figure 4-I).

In order to express GDNF instead of EGFP AAV 3TB-UTEB and AAV syn-GDNF-WB (figure 4-d) were cut with Age1 and Hind3. BB from the first and the GDNF sequence from the second were isolated by GE and ligated resulting in AAV UT-GDNF-B (figure 4-m).

\subsubsection{Double cassette AAV vector genomes}

To avoid the use of two vectors the two cassettes of the pSwitch system were cloned in a single vector with different configurations and promoters.

In order to clone the two cassettes into the same vector with the pSwitch protein under the control of the ubiquitous promoter Uptk the following steps were followed: AAVUptkpSwS was first cut with Spe1 and Sbf1, the two enzymes were heat inactivated, the DNA was treated with Klenow fragment, purified by PP and dephosphorilated. The plasmid containing the responsive cassette AAV UTEB was cut with Nsi1 and Mlu1, the enzymes were heat inactivated, the DNA was treated with the Klenow fragment and purified by GE in order to isolate the UTEB cassette. Being both fragments with blunt ends in was possible to perform a ligation which gave as a result two different configurations of the vector: AAV UptkpSwS-UTEB HtH (Head to Head; figure 4-n) and AAV UptkpSwS-UTEB HtT (Head to Tail; figure 4-o). 
In order to add a TB between the two cassettes of AAV UptkpSwS-UTEB HtH, the vector was cut with BamH1 and BsrG1 and purify by PP. AAV 1TB-UTEB was cut with Bgl2 and BsrG1 and the DNA fragment corresponding to the TB was isolated by GE. Bgl2 and BamH1 give compatible sticky ends and the BB and TB could be ligated resulting in AAV UptkpSwS-1TB-UTEB HtH (figure 4-p).

In order to restrict the expression to astrocytes AAV-GfaABC1D-pSw-S was first cut with Spe1 and then, after heat inactivation of the enzyme, the DNA was treated with the Klenow fragment; after a PP the vector was cut with Mlu1 and the DNA was isolated again by PP. The vector AAV UTEB was cut with Nsi1, the enzyme was heat inactivated and the DNA was treated with the Klenow fragment. After PP the DNA was cut with Mlu1 and the responsive cassette was isolated by GE. In this case the ligation between the backbone and the UTEB cassette was directional in a head to head configuration having both DNAs one blunt end and one sticky Mlu1 end. The final product was named AAV-GfaABC1DpSwS-UTEB (figure 4-q).

\subsubsection{AAV vector genomes for dopaminergic neuron generation}

Three vectors, delivered in bacteria, containing the TH1 (OCACo5052E1012D), AADC (OCAA05051B1155D), and VMAT-2 (IRCMp5012H034D) sequences were ordered from Imagenes; six primers with part of the sequences complementary to the three genes and with appropriate overhangs for cloning were ordered from sigma; an oligonucleotide (Oligo-D; table 3 ) that served as multicloning site (MCS) was ordered as well from Sigma in two separate sequences that were annealed.

Bacteria were seeded and repicked in order to isolate a single clone for each plasmid and minipreps were performed to isolate the plasmids.

The first cloning step consisted in inserting Oligo D which served as a MCS in an AAV vector; for this purpose AAV SEWB was cut with Age1 and Cla1, the BB was isolated by GE, the MCS was ligated, and the obtained vector was called AAV S-Oligo d-B.

In order to allow a single vector to produce two proteins an internal ribosome entry site (IRES) was used; AAV S-Oligo c-B and a vector carrying an IRES sequence pTR-UF22 were cut with Not1 and Cla1 and the IRES sequence, after being isolated by GE, was cloned into the AAV vector which was then purified by PP. The resulted plasmid was called AAV S-IRES-B.

The clone containing the TH1 gene obtained from Imagenes underwent a PCR amplification with the proper primers (table 2) using an annealing temperature of $72^{\circ} \mathrm{C}$ and a $\mathrm{MgCl}_{2}$ concentration of $1 \mu \mathrm{M}$. The PCR product was isolated by GE, cut with Sbf1 and EcoR1 and purified by PP. AAV S-IRES-B was cut with Sbf1 and EcoR1 and the BB was isolated from the EGFP-WPRE sequence by GE. The TH1 sequence was ligated in the AAV vector and the obtained vector was called AAV S-TH-IRES-B.

The PCR reaction (primers in table 2) for the AADC gene was performed with an annealing temperature of $68^{\circ} \mathrm{C}$ without $\mathrm{MgCl}_{2}$ and the product was isolated by GE. The purified DNA was then cut with Nhe1 and Hind3 and purified by PP. AAV-S-TH-IRES-B was cut as well with Nhe1 and Hind3 and the $B B$ was isolated by GE. AAAD DNA sequence was then cloned in the AAV BB and the obtained vector was named AAV-S-TH-IRES-AADC-B. 
The AADC gene was as well cloned in a vector without IRES; the same AADC fragment, cut with Nhe1 and Hind3, was inserted in AAV-S-IRES-B which was previously cut with the same enzymes and isolated by GE. The obtained vector was called AAV-S-AADC-B.

The vector containing VMAT-2 underwent a PCR reaction (primers in table 2) with $68^{\circ} \mathrm{C}$ as a annealing temperature and with $1 \mu \mathrm{M} \mathrm{MgCl}$ concentration. The PCR reaction produced two products due to a repetition in the sequence showing 2 distinct bands in the gel electrophoresis. The product of interest had a molecular size of $1600 \mathrm{bp}$. The correct band was then isolated by GE, cut with EcoR1 and Sbf1 and purified by PP. AAVS-IRES-B was cut with EcoR1 and Sbf1 and the backbone was purified by GE. The VMAT2 sequence and the AAV backbone were then ligated and the resulting vector was called AAV S-VMAT2-IRES-B.

To generate the vector AAV S-VMAT2-IRES-EGFP-B the vector AAV S-VMAT2-IRES-B and AAV SEWB were cut with Nhe1 and Hind3, the backbone from the first and the EGFP sequence from the second were isolated by GE; the two obtained pieces were ligated and bacteria were transformed.

The VMAT2 PCR product cut with EcoR1 and Sbf1 was used to generate AAV-S-VMAT2-B. AAV SOligoC-B was cut with the same enzymes and the backbone was isolated by PP. The two pieces were then ligated and bacteria were transformed.

All the clones were checked by DNA sequencing using primers (table 2) for Syn (forward), IRES (reverse), IRES (forward) and BGH (reverse).

\subsubsection{AAV vector genomes for control viruses}

The short version of the astrocytic promoter GFAP named Gfabc1d was kindly provided by Brenner (University of Alabama at Birmingham, Birmingham, Alabama) in a vector called ptyf 2xGfaABC1D. To excise the promoter BamH1 and Mlu1 were used and the desired DNA sequence was isolated by GE; AAV SEWB vector was cut with the same enzyme, the backbone was purified by GE, and the GfaABC1D promoter was inserted in the AAV vector obtaining the AAV-Gfabc1d-EWB vector (figure 4-b).

To obtain a vector expressing DsRed instead of EGFP under the synapsin promoter the DsRed DNA sequence was cut from the vector AAV HBADsRed with Hinc2 and BamH1, isolated by GE, and inserted in AAV SEWB in which the EGFP sequence was excluded by cutting the vector with Hinc2 and BamH1 and by purifying the backbone by GE; the obtained vector was named AAV SDsRedWB (figure 4-c).

\subsection{Viral vectors production and purification}

Vectors for the pSwitch experiment were packed both in AAV5 and AAV6 while all the clones for the dopaminergic neurons experiment were packed exclusively in AAV6 capsid.

\subsubsection{Continuous HEK-293 cell culture}

AAV are small dependovirus that are not able to replicate themselves without the presence of other viruses such Herpesvirus, Adenovirus or vaccinia virus, which provide the essential proteins for the replication cycle and the capsid.

For the production of AAV infective particles is then necessary to co-transfect HEK-293 cells with two constructs [102]: a first one containing the genes/cassettes between the two ITRs and a second one 
encoding the proteins for the capsid; the adenovirus protein E1 essential for packaging is constitutively expressed by HEK-293 cells.

HEK-293 cells were cultured in DMEM $10 \% \mathrm{FCS}, 1 \% \mathrm{PS}$ at $37^{\circ} \mathrm{C}$ with $5 \% \mathrm{CO}_{2}$ and $95 \%$ humidity. Depending on the volume of the culture, $175 \mathrm{~cm}^{2}$ culture flasks or 4 layers cell factories (CF, Nunc) were used. Cells were split once they reached $50-60 \%$ confluence; referring to the volume for $175 \mathrm{~cm}^{2}$ flasks (and in brackets for CF), cells were first washed with 7-8 $\mathrm{ml}$ (300-400 $\mathrm{ml}$ ) room temperature PBS and then trypsinized with $4 \mathrm{ml}(100 \mathrm{ml}) 0,5 \%$ trypsin $0,02 \%$ EDTA in PBS for 5 min at $37^{\circ} \mathrm{C}$. Cells detachment was helped by tapping mildly the flask and the CF. Trypsin activity was then stopped by adding $7 \mathrm{ml}$ ( $100 \mathrm{ml}$ ) of DMEM 10\% FCS 1\% PS; cells were then collected and centrifuged at $1400 \mathrm{~g}$ for $5 \mathrm{~min}$. Supernatant was discarded and cells were resuspended in culture media and counted using a hemocytometer; $2 * 10^{6}$ cells were diluted in $20 \mathrm{ml}$ culture media for seeding them in $175 \mathrm{~cm}^{2}$ flasks while $4 * 10^{7}$ cells were diluted in $500 \mathrm{ml}$ for seeding in CF for further expansion. When CFs were seeded for transfection and virus production, $5^{*} 10^{7}$ cells were used.

After approximately 48 hours cells in CF reach the confluency of $50-60 \%$, the optimal state for transfection and virus production.

\subsubsection{Calcium phosphate transfection of HEK-293 cells}

Depending on the serotype and on the amount of virus desired one or more cell factories were used for the same virus production; being AAV6 easily packed only one CF was used for this serotype while for AAV5 vectors normally three or four CF were used for each production. The volumes mentioned here refer to a single CF.

Before transfection the CF was washed once with DMEM without additives to remove the PS and excess FCS which can interfere with the transfection efficiency.

The transfection mix was then prepared by mixing $265 \mu \mathrm{g}$ of the AAV vector, $1 \mathrm{mg}$ of packaging vector and $1650 \mu \mathrm{l}$ of $2.5 \mathrm{M} \mathrm{CaCl}_{2}$ in a total volume of $16.5 \mathrm{ml}$ of sterile water. Afterwards $16.5 \mathrm{ml}$ of 2 $x$ HeBS ( $\mathrm{pH} 7.05$ ) was added to the transfection mix and incubated for exactly $55 \mathrm{sec}$ at room temperature. During this time the DNA associates with the calcium particles which are then taken by cells [103]. The reaction is stopped by adding the transfection mix to $315 \mathrm{ml} \mathrm{DMEM} 2 \%$ FCS without antibiotics. The transfection medium is then added to the cells which were then incubated for 10-16 hours at $37^{\circ} \mathrm{C}, 5 \% \mathrm{CO}_{2}$ and $95 \%$ humidity. The medium was then exchanged with approx. $750 \mathrm{ml}$ of DMEM $10 \%$ FCS, $1 \%$ PS. After approximately $36-40$ hours is possible to evaluate the efficiency of the transfection by checking the expression of the reporter genes present in the vectors.

After approximately 50 hours cells were harvested; after removing the medium from the CF cells were rinsed once with $300 \mathrm{ml}$ citric saline (CS) and then left in the incubator for 5-8 min with another $200 \mathrm{ml} \mathrm{CS}$ to promote the detachment of cells from the CF. The CF was then tapped, cells were collected, spun down for 5-10 min at $1300 \mathrm{rpm}$, resuspended in $20 \mathrm{ml}$ of tris-buffered saline pH8.5 and stored at $-80^{\circ} \mathrm{C}$ until further processing.

\subsubsection{AAV gradient centrifugation}

In order to lyse the cells and release the viral particles the cell suspension was thawed and frozen 3 times in ethanol/dry ice bath; after that $1.6 \mu \mathrm{l}$ of Benzonase $(12.5 \mathrm{U} / \mathrm{ml})$ was added to the tube and the mix was incubated for $30 \mathrm{~min}$ in a $37^{\circ} \mathrm{C}$ water bath. To help the lysis cells were slightly shaken every 5-10 min. After the incubation, the cell mix was centrifuged at $4000 \mathrm{rpm}$ at $18^{\circ} \mathrm{C}$ for $30 \mathrm{~min}$. 
The supernatants was carefully transferred to a new $50 \mathrm{ml}$ falcon tube and adjusted to a $30 \mathrm{ml}$ volume by addition of Tris-buffered saline.

The next step for virus isolation was to perform a gradient centrifugation [104]; using a pump catheter connected to a spinal needle $\left(20 \mathrm{G} \times 2^{3 / 4}\right)$ the iodixanol (Opti Prep ${ }^{\mathrm{TM}}$ ) step gradient was performed. The following stock solutions were prepared (E solution freshly prepared):

B - 10 x PBS-MK ( $80 \mathrm{~g} \mathrm{NaCl}, 2 \mathrm{~g} \mathrm{KCl}, 14.4 \mathrm{~g} \mathrm{Na}_{2} \mathrm{HPO}_{4}, 2.4 \mathrm{~g} \mathrm{KH}_{2} \mathrm{PO}_{4}$ dissolved in $990 \mathrm{ml}$ millipore sterile water, $\mathrm{pH} 7.4$; plus $5 \mathrm{ml} 2.5 \mathrm{M} \mathrm{KCl}$ and $5 \mathrm{ml} 1 \mathrm{M} \mathrm{MgCl}_{2}$, solution was sterile filtered).

C - 1x PBS-MK (500 $\mu \mathrm{l} 2.5 \mathrm{M} \mathrm{KCl}, 500 \mu \mathrm{l} 1 \mathrm{M} \mathrm{MgCl}_{2}$ in $499 \mathrm{ml}$ PBS)

D - $2 \mathrm{M} \mathrm{NaCl}$ in $1 \mathrm{x}$ PBS-MK

E - $54 \%$ working solution: ( $45 \mathrm{ml}$ iodixanol and $5 \mathrm{ml} \mathrm{B}$ ).

To obtain different iodixanol gradient solutions the solutions $C, D, E$, iodixanol and phenol red were intermixed as follow (volumes for 4 step gradient tubes):

$15 \%$ gradient: $15.0 \mathrm{ml} \mathrm{E}, 27.0 \mathrm{D}, 12.0 \mathrm{ml} \mathrm{C}$

$25 \%$ gradient: $12.2 \mathrm{ml} \mathrm{E}, 14.5 \mathrm{ml} \mathrm{C}, 150 \mu \mathrm{l}$ phenol red ( $0.05 \%$ in water)

$40 \%$ gradient: $20.0 \mathrm{ml} \mathrm{E}, 7.0 \mathrm{ml} \mathrm{C}$

$60 \%$ gradient: $25.0 \mathrm{ml}$ iodixanol, $150 \mu \mathrm{l}$ phenol red (0.05\% in water)

Fifteen $\mathrm{ml}$ of the supernatant was then transferred to a Quick seal tubes (Beckmann; $1 \times 3.5$ inch [25x $89 \mathrm{~mm}]$ ), using a $20 \mathrm{ml}$ syringe with a $20 \mathrm{G} \times 2^{3 / 4}$ spinal needle.

The iodixanol gradient solutions were added consecutively using the pump (10 $\mathrm{ml}$ of the $15 \%$ gradient, $6 \mathrm{ml}$ of the $25 \%$ gradient, $4 \mathrm{ml}$ of the $40 \%$ gradient, and $6 \mathrm{ml}$ or more of the $60 \%$ gradient to fill up the tube) into the Beckmann tubes underneath the virus solution. The tubes were then heatsealed and ultracentrifuged (SORVALL ${ }^{\circledR}$ Discovery 9OSE) for 1 hour and $15 \mathrm{~min}$ at $68000 \mathrm{rpm}$ ( $310.000 \mathrm{~g}$ ) at $18^{\circ} \mathrm{C}$ with acceleration " 5 " and deceleration " 0 ". After the centrifugation the viral particles are placed in the $40 \%$ gradient solution. To extract them, a needle was inserted at the interface of the $40 \%$ and $60 \%$ gradient solutions and the $40 \%$ gradient solution was extracted. Another similar needle was introduced on the top of the tube which allowed pressure equilibrium inside the tube. The virus-containing solution was collected in a $50 \mathrm{ml}$ falcon tube which was stored at $-20^{\circ} \mathrm{C}$ or used directly in the Fast Protein Liquid Chromatography step.

\subsubsection{Fast Protein Liquid Chromatography and dialysis (FPLC)}

The next purification step consisted in loading the virus into a Äkta-FPLC system (Amersham Biosciences) using a HiTrap ${ }^{\mathrm{TM}}$ Heparin HP $1 \mathrm{ml}$ column (GE Healthcare) in low salt solution (PBS-MK). The column was then washed with PBS-MK till the OD at $280 \mathrm{~nm}$ was below 0.0002 au (arbitrary units); after the washing step the virus was eluted in high salt conditions using $40 \%$ of PBS-MK $1 \mathrm{M}$ $\mathrm{NaCl}$. The AAV particles were further desalted by dialysis using Slide-A-Lyzer (MWCO $=10,000$; PIERCE) in 1 I PBS overnight, with 1 further hour of dialysis in 1 I of fresh PBS at $4^{\circ} \mathrm{C}$. The final virus solution was subjected to real-time PCR (RT-qPCR) quantification for the AAV-vector genome titre. 


\subsubsection{Virus DNA preparation for qPCR}

As every virus preparation is different, a estimation on the virus titre is needed; for this purpose a qPCR was performed. Briefly, $5 \mu \mathrm{l}$ of virus (or water for negative control) were subjected to DNAse digestion in $435 \mu \mathrm{l}$ water, $5 \mu \mathrm{l}$ DNAse I (10 units) and $50 \mu \mathrm{l} 10 \mathrm{x}$ DNAse I buffer for 1 hour in a $37^{\circ} \mathrm{C}$ water bath. Samples were then centrifuged for $1 \mathrm{~min}$ at max speed; next, $55 \mu \mathrm{l}$ of 10X proteinase $\mathrm{K}$ buffer and $5 \mu \mathrm{l}$ of Proteinase $\mathrm{K}(20 \mathrm{mg} / \mathrm{ml})$ were added to the tube and the sample was strongly mixed and incubated for 1 hour in a $37^{\circ} \mathrm{C}$ water bath. The solution was then centrifuged again at max speed for $1 \mathrm{~min}$ for phenol/chloroform extraction.

\subsubsection{Phenol/chloroform extraction:}

To further purify the DNA before the qPCR a phenol/chloroform extraction was performed: briefly $500 \mu \mathrm{l}$ of phenol equilibrated at RT was added to the sample which was then strongly mixed for 10 sec and centrifuged at max speed at RT for $1 \mathrm{~min}$. The aqueous phase was then transferred to a new $1.5 \mathrm{ml}$ eppendorf tube and added with $250 \mu \mathrm{l}$ of equilibrated phenol and $250 \mu \mathrm{l}$ of chloroform. The sample was again strongly mixed and centrifuged at max speed for $1 \mathrm{~min}$ at RT. The aqueous phase was transferred to $500 \mu \mathrm{l}$ chloroform in a new $1.5 \mathrm{ml}$ eppendorf tube, strongly mixed for $10 \mathrm{sec}$ and centrifuged for $1 \mathrm{~min}$ at max speed and RT. The aqueous phase was again transferred to a new $1.5 \mathrm{ml}$ eppendorf tube and along with $45 \mu \mathrm{l} 3 \mathrm{M}$ sodium-acetate $\mathrm{pH}$ 5.0-5.3, $1.5 \mu \mathrm{l}$ of glycogen for molecular biology and $1 \mathrm{ml}$ of cold $100 \% \mathrm{EtOH}$. The sample was mixed, put in dry ice for 5 min to help the DNA precipitation and centrifuged at max speed for $30 \mathrm{~min}$ at $4^{\circ} \mathrm{C}$. The extracted DNA pellet was washed with $1 \mathrm{ml}$ of cold $70 \%$ ethanol and centrifuged for $10 \mathrm{~min}$, the supernatant was removed. The pellet dried and re-dissolved in $200 \mu \mathrm{l}$ of TE $\mathrm{pH}$ 8.0.

\subsubsection{AAV qPCR}

The amount of PCR product in this type of qPCR was followed in real time by SYBR green incorporation in the dsDNA PCR product. All samples including the negative control were further diluted 1 to 20 before qPCR. The qPCR reaction was set up in $200 \mu \mathrm{l}$ "thin wall tubes" and contained $10 \mu \mathrm{l}$ mix (Platinum SYBR ${ }^{\circledast}$ Green qPCR SuperMix-UDG), $2 \mu \mathrm{l}$ of $4 \mathrm{mM}$ primer 1 and 2, $1 \mu \mathrm{l}$ fluorochrome flurescein, $4 \mu \mathrm{l}$ of extracted DNA, standard or positive control, plus $1 \mu \mathrm{H}$ H2O. Each unknown sample was assayed in duplicate and, when possible, with different primers depending on the vector (WPRE, bGH, Syn specific primers; table 2). qPCR was performed on "icycler" qPCR machine and analyzed with kcycler software. Amplification started with 15 min incubation at $98^{\circ} \mathrm{C}$ (to release the antibody bound to the polymerase) followed by 30 cycles of amplification (annealing 15 sec between $60^{\circ} \mathrm{C}$ and $72^{\circ} \mathrm{C}$, elongation for $20 \mathrm{sec} / \mathrm{kb}$ at $72^{\circ} \mathrm{C}$ and separation of DNA strands for 15 sec at $98^{\circ} \mathrm{C}$ ). After amplification a melting curve was performed with $0.5^{\circ} \mathrm{C}$ increase each 30 seconds until reaching $98^{\circ} \mathrm{C}$. The melting curve is an indicator of PCR amplification product specificity. Cycler was programmed to stay at on hold at $18^{\circ} \mathrm{C}$ after the melting curve. Concentrations were automatically calculated by a regression curve between the threshold cycle and the known amounts of standards in logarithmic scale. Threshold cycle for known concentration samples was used to extrapolate the pg of DNA in unknown samples.

\subsubsection{Calculation of AAV viral genomes}

The correlation between amounts of double stranded DNA used and the qPCR standard (dependent on molecule size) and the single stranded DNA present in the virus genome was assumed to be 200 pg of ssDNA AAV genome equal to $5.2 \times 10^{7}$ genomes $\left(0.026 \times 10^{7}\right.$ genomes/pg of ssDNA). 
Titre genome was calculated as follows:

(value in $\mathrm{pg}$ ) $\mathrm{Z} \mathrm{ZYY} \times 0.026 \times 10^{7} / \mathrm{X} / \mathrm{n}=$ genomes $/ \mu$ l (often referred as vg, viral genome).

Where $Z$ corresponds to the ssDNA sample dilution before qPCR (usually 20 ), $Y$ is the volume where the ssDNA was dissolved after ethanol precipitation, $X$ is volume in $\mu l$ of virus used for DNA preparation and $\mathrm{n}$ is the volume in $\mu \mathrm{l}$ applied in the $\mathrm{qPCR}$ tube for quantitative determination.

Assuming that only one every $30^{\text {th }}$ viral genome is infectious, the obtained genomes were divided by 30 to obtain the transduction units values that were used as reference in each in vitro transduction or in vivo injection.

Finally, the purity of the vectors was determined by SDS-gel electrophoresis followed by coomassie brilliant blue staining. In short, $5 \mu$ l of virus were mixed with $6 x$ SDS sample buffer and boiled for 5 $\min$ at $95^{\circ} \mathrm{C}$. Denaturated virus capsid proteins were loaded in $10 \%$ polyacrylamide/bis-acrylamide gels and were run until the bromophenol blue reached the bottom of the resolving gel. Next coomassie brilliant blue staining was performed using standard protocols (figure 5). Aliquots of the $\mathrm{AAV}$ vectors were made and stored at $-80^{\circ} \mathrm{C}$ until required. During all procedures dealing with AAV vectors, $0.5 \%$ SDS solution in water was used for disinfection.

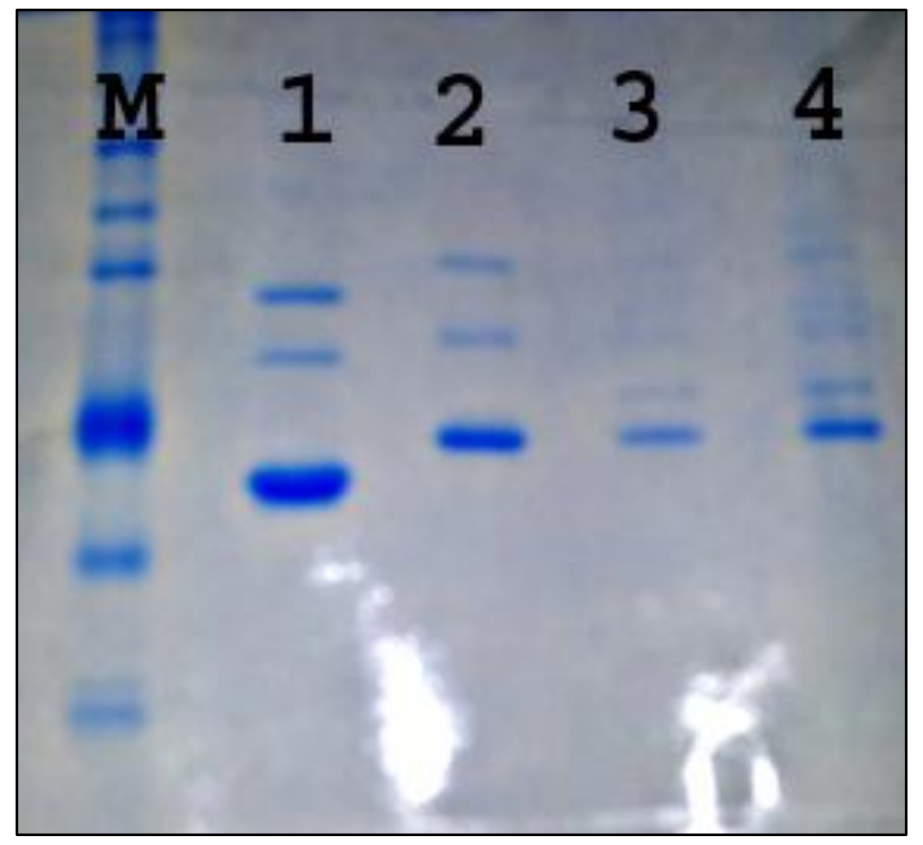

Figure 5) SDS-gel electrophoresis of AAVs followed by coomassie brilliant blue staining. $M=$ broad range protein marker; 1 = AAV6; 2 = AAV5 (good preparation); 3 = AAV5 (low titre); 4 = AAV5 (some contamination in the sample).

\subsection{Cell culture}

Primary cortical neurons in culture were used both for a rapid screening of the vectors with the pSwitch system and for the dopamine producing neurons project. AAV5 serotype is not able to infect neurons in culture so only AAV6 vectors were used. ; before seeding (and infecting) the neurons is necessary to coat the culture plates with different attachment factors creating the physiologically relevant in vitro conditions to support normal cell growth function and ensure optimized cell culture conditions. 


\subsubsection{Coating of culture plates for primary cortical cells culture}

Cortical neurons were plated in 24 and 96 wheels/plate and, if immunohistochemistry was planned, $13 \mathrm{~mm}$ cover slips were sterilized by soaking them in ethanol, flamed, and placed into the 24 wells/plate. 24 wells/plates were first incubated for 12 to $24 \mathrm{~h}$ at RT with P-ORN (1 $\mathrm{\mu g} / \mathrm{ml})$ in sterile $\mathrm{H}_{2} \mathrm{O}\left(500 \mu \mathrm{l} /\right.$ well). After washing twice with $500 \mu \mathrm{l} /$ well sterile $\mathrm{H}_{2} \mathrm{O}$, plates were incubated with laminin 1:1000 in NBM $(1 \mathrm{\mu g} / \mathrm{ml}, 500 \mu \mathrm{l} /$ well $)$ overnight at $37^{\circ} \mathrm{C}, 5 \% \mathrm{CO}_{2}, 95 \%$ humidity. Before transferring cells into wells, plates were washed twice with sterile NBM $(500 \mu \mathrm{l} /$ well), then $500 \mu \mathrm{l}$ of $\mathrm{HCN}$ medium were added and the plates were kept at $37^{\circ} \mathrm{C}$. The procedure for the 96 wells/plate was the same but the volumes used was $100 \mu \mathrm{l} /$ well instead of $500 \mu \mathrm{l} /$ well.

\subsubsection{Primary cortical cells culture}

Primary cortical neuron cultures were obtained by dissecting the cortex of E18 (Embryonic day 18) Wistar rats which were processed as previously described [105].

All surgical procedures were performed on ice. Briefly, cortex tissue pieces were collected in ice-cold $\mathrm{CMF}$ medium and centrifuged at $800 \mathrm{rpm}(73 \mathrm{~g})$ for $4 \mathrm{~min}$ at $4^{\circ} \mathrm{C}$. The medium was then removed and the pellet was incubated in $750 \mu \mathrm{l}$ trypsin $\left(0.25 \%, 15 \mathrm{~min}, 37^{\circ} \mathrm{C}\right)$; trypsin activity was stopped by addition of $700 \mu \mathrm{l}$ ice-cold FCS and $25 \mu \mathrm{l}$ DNAse was added to dissolve DNA-aggregates released from damaged cells. The pellet was then mechanically dissociated by pipetting the mixture gently through a fire-polished Pasteur pipette. After another centrifugation at $800 \mathrm{rpm}$ for $4 \mathrm{~min}$, the pellet was resuspended in warm HCN culture medium. Cells were seeded in poly-L-ornithine/laminin culture plates at a density 250.000 cells/well in $750 \mu \mathrm{l}$ media for the 24 wells/plates and at a density 50.000 cells/well in $100 \mu \mathrm{l}$ for the 96 wells/plate. Cultures were maintained at $37^{\circ} \mathrm{C}$ in $5 \% \mathrm{CO}_{2}$ and $95 \%$ humidity in $\mathrm{HCN}$ medium; medium was normally refreshed every 4-5 days by discarding, in the 24 wells/plate, $200 \mu \mathrm{l} /$ well and adding $300 \mu \mathrm{l} /$ well of fresh media which was previously $\mathrm{pH}$ equilibrated by leaving it over night in the incubator. For the 96 wells/plate $25 \mu \mathrm{l} /$ well were discarded and 50 $\mu \mathrm{l} /$ well were added. Neurons can stay in these conditions for up to 3 weeks.

\subsubsection{Infection of primary cortical neurons}

Cortical neurons were left to adapt to the culture conditions for at least 2 days (DIV2 $=$ day in vitro two). To infect neurons in the 24 wells/plate, $10 \mu \mathrm{l}$ of the desired amount of virus diluted in PBS was added to each well. For 96 wells/plate neuron infection, $2 \mu$ of the desired amount of virus diluted in PBS was added to each well.

The expression of the proteins encoded by the viruses starts approximately 36 hours after infection and reaches its full expression after 96 hours.

When live neurons expressing EGFP were imaged, an incubator chamber $\left(37^{\circ} \mathrm{C}\right.$ and $\left.5 \% \mathrm{CO}_{2}\right)$ was mounted in an Olympus inverted microscope and pictures were acquired using Olympus Axiovision rel. 4.7 software.

\subsubsection{Western blot}

To verify the protein expression after virus infection western blots [106] were performed on neurons seeded in 24 wells/plates.

\subsubsection{Cell lysis}

Neurons were first washed with $500 \mu \mathrm{l}$ of ice cold PBS and were then treated on ice with lysis buffer (see solutions); wells were scratched with a cut tip to detach cells and the mixture was transferred to 
a $1.5 \mathrm{ml}$ eppendorf tube on ice. After spinning down the cells with $30 \mathrm{sec}$ centrifugation at max speed at $4^{\circ} \mathrm{C}$ samples were sonicated twice for $15 \mathrm{sec}$ at $40 \%$ power (UW 2070 BANDELIN electronic; Berlin). Tubes were then centrifuged for $30 \mathrm{~min}$ at max speed at $4^{\circ} \mathrm{C}$ and the supernatant containing the proteins was transferred to a new tube (the pellet was saved as well in case of proteins associated with the membranes).

To compare the relative amount of a protein in different samples is essential to perform the western blot with a comparable total amount of proteins. To estimate the protein concentration after lysis the BCA (bicinchoninic acid) test was performed.

\subsubsection{BCA test for protein concentration determination}

$\mathrm{BCA}$ test, or Lowry assay, is a colorimetric test based on the ability of proteins in basic condition to reduce $\mathrm{Cu}^{2+}$ into $\mathrm{Cu}^{+}$. These ions form complexes with 2 molecules of bicinchoninic acid (BCA) leading to the formation of a violet compound with maximum absorbance at $562 \mathrm{~nm}$. The amount of product will be proportional to the proteins concentration; therefore, comparing the absorbance of the unknown sample with the absorbance in a standard curve made with known samples we can extrapolate the protein concentration.

Samples were diluted 1 to 10 in PBS and $10 \mu \mathrm{l} /$ well of it were transferred in a 96 well plate; the standard curve was done by preparing samples of BSA ranging from $4 \mathrm{mg} / \mu \mathrm{l}$ to $0.031 \mathrm{mg} / \mu \mathrm{l}$ in a serial dilution of 8 standard diluted each time twice with PBS; $10 \mu \mathrm{l} /$ well of each standard were then transferred to the same 96 well/plate. Both the standard and the samples were done in double. The reaction starts by adding to each well $190 \mu$ l of reagent A and B previously mixed at a ratio of 50 to 1 . The plate was then incubated at $37^{\circ} \mathrm{C}$ and the absorbance was taken using a TECAN Rainbow plate reader and Magellan software (V3.11) after 2 hours. Data were interpolated using Microsoft excel 2003 and the time-point with the best standard curve was used to calculate the protein concentrations of the unknown samples.

\subsubsection{SDS polyacrylamide gel electrophoresis (SDS-PAGE)}

To separate proteins a two-phase polyacrylamide gel was used with a polyacrylamide concentration of $12 \%$ (resolving phase) and a $5 \%$ (stacking phase).

Upon fresh preparation, the $12 \%$ polyacryamide phase (see solutions) was added to $0.75 \mathrm{~mm}$ racks and a layer of isopropanol was carefully added on the top of the phase to level it. Once dried, the isopropanol was discarded and the mix for the stacking gel (see solutions) was added in presence of a suitable comb.

Once dried the rack was mounted in the electrophoresis chamber in presence of running buffer. Equal amount of proteins for each sample were diluted in PBS and 6X SDS Loading Buffer, heated for $5 \mathrm{~min}$ at $95^{\circ} \mathrm{C}$, let to cool down at room temperature and loaded into the gel; to define a molecular

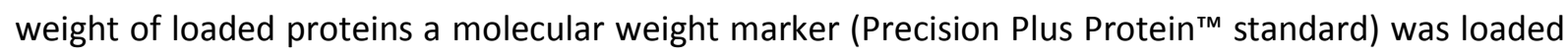
and run in parallel.

To allow the samples to enter into resolving gel without smearing the current applied for the first 15 min was $7 \mathrm{~mA} / \mathrm{gel}$ (maximum electric field of $90 \mathrm{~V}$ ). For the run in the resolving gel the current was $15 \mathrm{~mA} /$ gel (maximum electric field of $150 \mathrm{~V}$ ) and the run lasted until the bromophenol blue reached the bottom of the gel. Separated proteins were then transferred to a nitrocellulose membrane for immunostaining. 


\subsubsection{Immunoblotting}

The Mini Trans-Blot Cell set up was used to transfer proteins from the polyacrylamide gels to the nitrocellulose membranes. The transfer sandwich was assembled by orientating cathode, fibber pads, Whatman filter paper, gel, membrane, Whatman filter paper, filter pads and anode so protein transfer goes in the direction of cathode to anode. The nitrocellulose membrane, the Whatman filter papers and fibber pads were preliminary soaked in transfer buffer. The "sandwich" was placed in the Mini Trans-Blot Cell and a current of $360 \mathrm{~mA}$ or a maximum voltage of $160 \mathrm{~V} /$ gel was applied for 70 $\min$ at $4^{\circ} \mathrm{C}$.

The efficiency of the transfer was confirmed by immersing the nitrocellulose membrane for few seconds in Pancean S which highlights all proteins; after this control the membrane was washed with water and TBS-T.

To avoid unspecific binding of the antibodies the membrane was blocked using TBS-T 5\% fat milk for 1 hour at RT under shaking. The membrane was then incubated over night at $4^{\circ} \mathrm{C}$ under shaking with the desired primary antibody in the appropriate dilution (see table) in TBS-T 5\% fat milk. The membrane was then washed three times for 15 min under shaking with TBS-T and was incubated with the appropriate secondary antibody, coupled to Horseradish peroxidase (HRP), for one hour at RT under shaking; after another three 15 min washing with TBS-T at RT the membrane was ready for imaging.

To visualize the bands on the membrane the substrate of HRP, luminol, has to be added to the membrane. This compound, reacting with the HRP, gives rise to chemiluminescence. To promote the reaction solutions ECL-1 and 2 were prepared, mixed in equal volume, and added to the membrane. The luminescence was then visualized and measured using the Fluor-STM-Max Gel Imager and Quantity One software (version 4.2.1). The solutions and the reaction have to be stored and performed in dark conditions.

\begin{tabular}{|c|c|c|c|c|}
\hline Antibody & Species & Dilution & Company & Ord. number \\
\hline \multicolumn{5}{|l|}{ Primary antibody } \\
\hline Anti-TH & $\begin{array}{c}\text { Mouse } \\
\text { monoclonal }\end{array}$ & $1: 1000$ & Chemicon Millipore & ab 152 \\
\hline Anti-AADC & $\begin{array}{c}\text { Rabbit } \\
\text { polyclonal }\end{array}$ & $1: 1000$ & Abcam & ab 49916 \\
\hline Anti-VMAT-2 & $\begin{array}{l}\text { Rabbit } \\
\text { polyclonal }\end{array}$ & $1: 3000$ & Abcam & ab 81855 \\
\hline Anti-GAPDH & $\begin{array}{c}\text { Mouse } \\
\text { monoclonal }\end{array}$ & 1:10000 & Biotrent & $5 \mathrm{G} 4$ \\
\hline Anti- $\alpha$-synuclein & $\begin{array}{c}\text { Mouse } \\
\text { monoclonal }\end{array}$ & $1: 1000$ & Invitrogen & 328100 \\
\hline \multicolumn{5}{|c|}{ Secondary antibody } \\
\hline HRP-anti rabbit & Donkey & $1: 3000$ & DIANOVA & 711-035-152 \\
\hline HRP-Anti mouse & Donkey & $1: 3000$ & DIANOVA & $715-035-150$ \\
\hline
\end{tabular}

Table 4) Summary of antibodies used for western blot. TH = tyrosine hydroxylase; AADC = aromatic amino acid decarboxylase; VMAT-2 = Vesicular monoamine transporter 2; GAPDH = Glyceraldehyde 3-phosphate dehydrogenase.

\subsubsection{Viability test using primary cortical neurons}

To asses possible toxic effects of viruses, proteins or other compounds a viability test was performed using the WST-1 proliferation reagent. The assay is based on the cleavage of the tetrazolium salt WST-1 by succinate-tetrazolium reductase producing soluble formazan. The enzyme is part of the 
respiratory chain in mitochondria and the conversion only occurs in viable cells. The amount of formed formazan dye, which absorbance can be measured at $450 \mathrm{~nm}$, will be then proportional to viable cells.

For the test, performed in neurons cultured in 96 wells/plate, the WST-1 reagent was diluted in HCN media (1:10) and $50 \mu \mathrm{l}$ of the solution were added to the neurons and to empty wells (to be used as blank); plates were then incubated at $37^{\circ} \mathrm{C}$ and the absorbance at $450 \mathrm{~nm}$ was measured after 30 min, 2 and 4 hours with a microplate reader (Tecan RainBow). To evaluate the neurons viability the blank value was subtracted from the obtained values and tested neurons were compared in percentage with not treated neurons. The time-point which gave less variability was used.

\subsubsection{HPLC with cell lysates of primary cortical neurons}

To determine the amount of Dopamine, DOPAC and homovanillic Acid (HVA) produced from cells an HPLC (high pressure liquid chromatography) system coupled with an electrochemical detector was used. Both the released and the not released fraction of catecholamines were measured.

To analyze the released catecholamines $400 \mu \mathrm{l}$ of the media were taken and diluted in $50 \mu \mathrm{l}$ of $2 \mathrm{M}$ perchloric acid (PCA) and $50 \mu \mathrm{l}$ of $1 \%$ sodium metabisulfite $\left(\mathrm{Na}_{2} \mathrm{~S}_{2} \mathrm{O}_{5}\right)$; the mixture was kept on ice for few minutes and centrifuged at maximum speed for $10 \mathrm{~min}$ at $4^{\circ} \mathrm{C}$. The supernatant was then filtered using Minisart SRP4 filters (Sartorius stedim biotech) and the samples were then directly loaded in the HPLC.

To extract the not released fraction of dopamine the left over media was carefully removed and the cells were incubated for $10 \mathrm{~min}$ at room temperature with $200 \mu \mathrm{l}$ of $3 \%$ trichloroacetic acid (TCA); $180 \mu \mathrm{l}$ of TCA were then transferred to $10 \mu \mathrm{l}$ of $2 \mathrm{M} \mathrm{PCA}$ and $10 \mu \mathrm{l}$ of $1 \%$ sodium metabilsulfide, incubated on ice for few minutes and centrifuged for $10 \mathrm{~min}$ at max speed at $4^{\circ} \mathrm{C}$. The supernatant was then filtered using the Minisart SRP4 filter and used for HPLC.

Two series of 4 standards for the external and internal fraction were prepared. To minimize the variation the two series of measurement were performed mimicking the samples composition with known amount of Dopamine, DOPAC and HVA; for a $1 \mathrm{ml}$ solution $100 \mu \mathrm{l}$ were $2 \mathrm{M}$ PCA, $100 \mu \mathrm{l}$ were $1 \%$ sodium metabisulfite and the leftover $800 \mu$ l were culture medium for the external fraction and $3 \%$ TCA for the internal fraction in which the catecholamines were diluted in order to obtain a final concentration of $0,0.15,0.3$ and $1.5 \mu \mathrm{M}$ (same concentration for each compound).

The HPLC system consisted of a Guard cell (model 5020, ESA) that was kept at $600 \mathrm{mV}$ to oxidize the

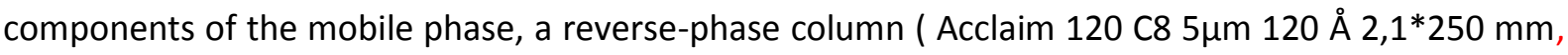
Bischoff, Leondberg, Germany) and an ESA electrochemical detector Coulochem II equipped with a 5011A analytical cell.

Standards and samples were transferred to HPLC vials and loaded to the HPLC autosampler which was kept at $6^{\circ} \mathrm{C}$ and the HPLC software was programmed to inject $20 \mu \mathrm{l}$ of the samples to the column. The flow rate of the mobile phase (see solutions) was $0.4 \mathrm{ml} / \mathrm{min}$ and the run lasted for 22 $\mathrm{min}$. The guard cell was held at $600 \mathrm{mV}$ while the first channel of the analytical cell was held at $50 \mathrm{mV}$ to oxidize non-specific components and the second one at $300 \mathrm{mV}$ and used for quantification. To minimize errors, standards were run every 12-15 samples and averaged. The amount of the 3 compounds was calculated by integrating the areas under the peaks. The retention time for DOPAC was around $4.8 \mathrm{~min}$, for Dopamine $5.7 \mathrm{~min}$ and for HVA $10.8 \mathrm{~min}$. 


\subsubsection{Live cell imaging}

Two different fluorescence microscopes were used for imaging depending on whether live neurons were imaged or if immunohistochemistry was performed.

When imaging live neurons a Zeiss Axiovert 200M inverted microscope equipped with an incubator chamber to keep the temperature at $37^{\circ} \mathrm{C}$ and $5 \% \mathrm{CO}_{2}$ was used; neurons in 24 or $96 /$ wells plate were placed in the chamber and Images were acquired with a CCD camera (Axiocam b/w) and with Axiovision Rel 4.7 software. Further processing of the images was done using Adobe Photoshop 7.0.

When our interest was to perform immunohistochemistry (IHC) to verify protein expression 24 wells/plates with cover slips were used.

Neurons were washed twice with ice-cold PBS and were then incubated with ice-cold $4 \%$ paraformaldeide in PBS (PFA see 2.2 "solutions") for 8 minutes; the PFA cross-links the protein inside cells preserving the structures allowing the antibody to recognize the antigen of interest.

After fixation neurons were washed twice with ice-cold PBS and were incubated with $10 \%$ NGS, $0.3 \%$ triton in PBS for 30 minutes at $37^{\circ} \mathrm{C}$ for blocking. Cells were then incubated with the primary antibody (table 5) diluted in PBS $2 \%$ NGS and $0.3 \%$ triton for 90 minutes at $37^{\circ} \mathrm{C}$. After 3 washing step of 5 min each with PBS neurons were incubated with the appropriate secondary antibody (table 5) diluted in PBS $2 \%$ NGS for $90 \mathrm{~min}$ at $37^{\circ} \mathrm{C}$. Neurons were washed another 3 times with PBS and incubated for 5 min at room temperature with DAPI 1:50000 in PBS and washed another 2 times with PBS. A last washing step was done with bi-distilled water and the cover slips were then mounted face-down on slides using a drop of Mowiol; The slides were let to dry over-night and could then be imaged using an Olympus Axioplan 2 microscope equipped with a CCD (Axiocam HRm) camera and the Axiovision 4.7 software. Further processing of images was done using Adobe Photoshop 7.0.

\begin{tabular}{|l|c|c|c|c|}
\hline \multicolumn{1}{|c|}{ Antigen } & Species & dilution & Company & Ord. number \\
\hline Primary antibody & & & & \\
\hline Anti TH & Mouse mono & $1: 1000$ & Chemicon Millipore & ab 152 \\
\hline Anti AAAD & Rabbit poli & $1: 1000$ & Abcam & ab 49916 \\
\hline Anti VMAT2 & Rabbit poli & $1: 3000$ & Abcam & ab 81855 \\
\hline Secondary antibody & & & & \\
\hline Cy 3 anti rabbit & Goat & $1: 250$ & Dlanova & $111-165-006$ \\
\hline Cy 3 anti mouse & Goat & $1: 250$ & Dianova & $115-165-003$ \\
\hline
\end{tabular}

Table 5) Summary of antibodies used for western blot. TH = tyrosine hydroxylase; AADC = aromatic amino acid decarboxylase; VMAT-2 = Vesicular monoamine transporter 2;

\subsection{Animal surgery}

All animals operations were performed under grant agreement $n^{\circ}$ HEALTH-2008-222925 (AM, JT) and according to the regulations of the local animal research council, legislation of the State of Lower Saxony (Braunschweig) and the European Community Council Directive of $24^{\text {th }}$ November 1986 (86/609/EEC).

Both mice and rats were kept in a 12 hours cycle day/night with access to food and drink ab libitum if not specified.

Animals were provided with methamizol $(1.5 \mathrm{mg} / \mathrm{ml})$ in the drinking water 3 days prior the operation and for an additional week after it. 


\subsubsection{Cranial window implantation in mice}

The procedure is based on the protocol described by Mostany [107]. Female NMRI or C57/BI6 mice between 25 and 35 grams were anesthetized with a mixture of $80-100 \mathrm{mg} / \mathrm{kg}$ of ketanest and 5-10 $\mathrm{mg} / \mathrm{kg}$ of xilazin. One third of the dosage was given every $30 \mathrm{~min}$ to keep the animal sleeping if the operation lasted longer. The animal was then shaved and fixed by ear bars and a jaw holder in a stereotaxic frame (Kopf instrument); the eyes were protected using bepanthene and the head was cleaned and disinfected using $70 \%$ ethanol and braunol. The skin covering the skull was removed by cutting an oval shape from the imaginary line connecting the eyes and the scalp and exposing the whole flat area of the skull between the bregma and the lambda. The connective tissue covering the skull was then removed, blood capillaries were cauterized and the bone was scratched with a scalpel and dried to help the dental cement to attach. Using a mini-driller (Netzgerät AC/DC Typ NG 2-S: Praxxon) with a $0.8 \mathrm{~mm}$ tip (Dremel) a hole with $\sim 4 \mathrm{~mm}$ diameter was drilled in the left hemisphere between the lambda and the bregma. This step is crucial and has to be done carefully to avoid the bleeding of the dura mater that will obscure the cranial window. Once the bone was removed the area was rinsed with PBS and cotton buds.

Once cleaned, one to three points of injection were defined; injection through blood vessels was avoided. A glass capillary filled with mineral oil, a bubble of air and the virus mix in PBS was mounted in a nanoliter 2000 injector coupled with a micro4 smart controller to better monitor the injection. One $\mu \mathrm{l}$ of the virus mix was injected in each point with an injection speed of $200 \mathrm{nl} / \mathrm{min}$; the glass capillary was left in place for 3 minutes before and after the injection to allow the tissue around the point of injection to adapt to the capillary and to absorb the virus. The deepness of injection ranged between 0.2 and $0.5 \mathrm{~mm}$ depending on the observed reflux that was taking place during the injection; these depths correspond to the $3^{\text {rd }}-4^{\text {th }}$ layer in mice cortex (figure 6).

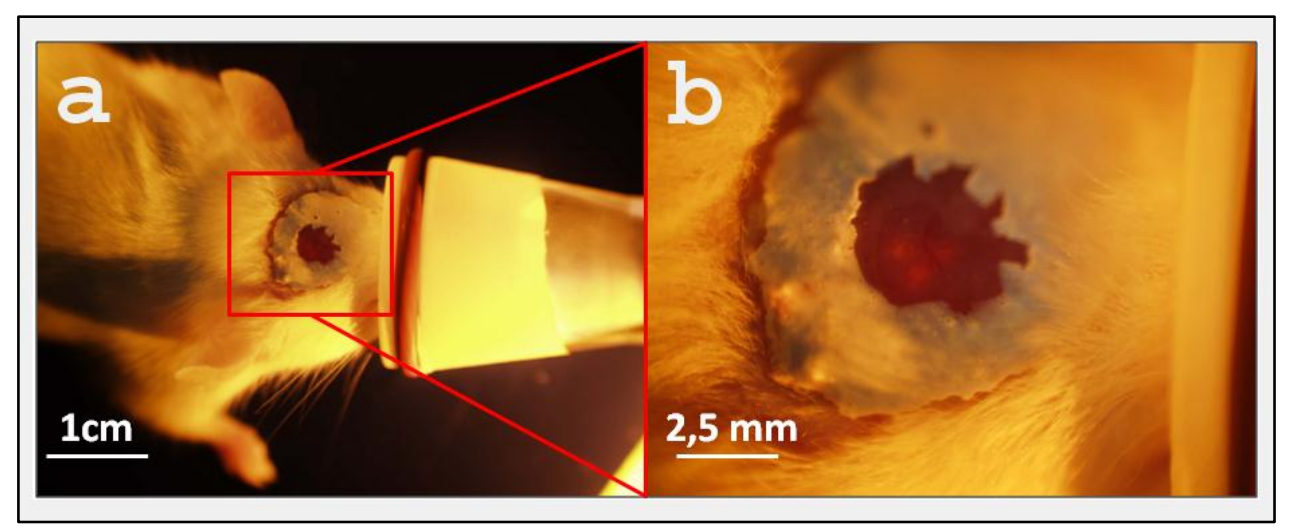

Figure 6) Cranial window inplantation in mice. a) Overview of the anesthetized animal with a cranial window implanted in the OV110 microscope chamber. Details of the cranial window; $b$ ) the three points of injection can be identified by the red fluorescence dots given by a AAV6 SDsRedWB vector. $S=\operatorname{sinapsin}$ promoter, $W=$ WPRE; $B=$ BghpA.

The cortex was then cleaned with PBS and a $5 \mathrm{~mm}$ cover slips was placed over the cranial window and glued using histoacrylic glue. The glue is toxic for the neurons, so special care was taken to ensure that the glue stays only between the glass and the bone and that does not enter in contact with the brain. This is achieved by pressing the window with a forceps and adding few $\mu$ l of glue per time from the side of the glass. Once the glass was sealed, the glue was used to cover the rest of the skull and the interface between the skull and the skin. After a minute the glue is dry and the exposed skull can be covered with dental cement. The animal was then kept in a single animal cage and after 2 weeks was ready for imaging. 
To induce the pSwitch system animals were injected intraperitoneally for three consecutive days with 20 or $10 \mathrm{mg} / \mathrm{kg} /$ day of mifepristone diluted in DMSO at a concentration of $5 \mathrm{mg} / \mathrm{ml}$; imaging sessions were performed the third day after the last injection.

\subsubsection{Two-photon imaging in mice}

For the two-photon (2-p) imaging a custom made microscope kindly provided by the MPI of experimental medicine (Göttingen) was used.

The microscope is equipped with a fs-pulsed titanium-sapphire laser (Chameleon Ultra II; Coherent, Glasgow, UK) and the Apochromat 20X/1.0 DIC objective. For excitation the laser was set at $925 \mathrm{~nm}$, the emitted light was split with a $560 \mathrm{~nm}$ long-pass filter (Carl Zeiss) end the EGFP signal was acquired with photomultiplier tube (Hamamatsu, Japan) through a $542 \pm 27 \mathrm{~nm}$ band pass filter (Semrock).

The animal was anesthetized and was kept asleep with $5 \%$ and $2 \%$ isofluoran. Using dental cement, a metal bar with screw holes was fixed on the right side of the skull. The metal bar was then screwed to a custom made frame with a heating system connected to a rectal probe to maintain the temperature of the animal at $37^{\circ} \mathrm{C}$.

The areas of injection were identified using an UV light with a 5 X/NA 0.15 objective (Carl Zeiss, Jena, Germany); once the area was defined a 20x objective was mounted and a 2-p image was taken. Images were acquired as a Z-stack with $2.4 \mu \mathrm{m}$ distance between consecutive images and the laser power was adjusted depending on the depth. The best settings for each animal (laser power, deepness and PMT power) were defined at the first imaging session and kept the same for the next sessions. Images were then processed using Image J and Imaris.

\subsubsection{OV-100 imaging in mice}

The Olympus OV-100 Small Animal Imaging system (Olympus corp.) microscope was kindly provided by Johannes Wessels (nephrology department, Universitätmedizin Göttingen). The microscope is equipped with an MT-20 light source (Olympus Biosystem, Planegg, Germany) and a DP70 CCD camera (Olympus). Images were captured directly on a personal computer (Fujitsu Siemens Computers, Munich, Germany) with the LM 2.8 software (Olympus)

The animal was anesthetized and was kept asleep with $5 \%$ and $2 \%$ isofluoran, respectively, in the imaging chamber. Illumination and acquisitions settings were defined at the first imaging session for each animal and kept the same at the different time points.

Images were processed using ImageJ [108] and, to quantify the fluorescence, the intensity in the area of injection was measured and normalized versus the background; the intensity was then plotted in a graph considering as " 1 " the background fluorescence (figure 7). 


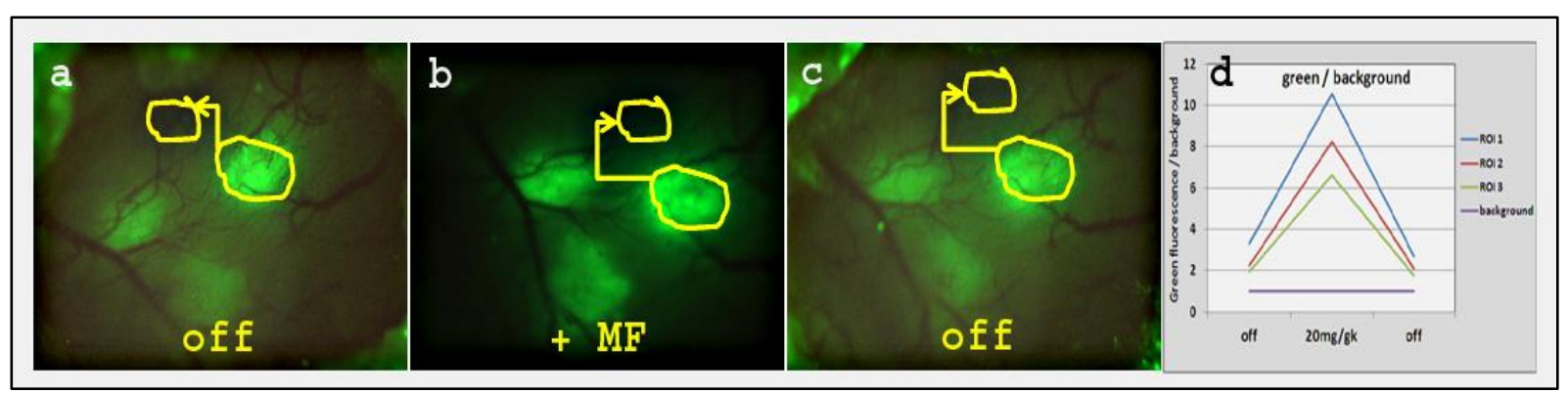

Figure 7) OV 100 analysis: the same three points of injection can be easily defined when the system is off (picture a), induced with $20 \mathrm{mg} / \mathrm{kg} \mathrm{MF} \mathrm{(picture} \mathrm{b),} \mathrm{and} \mathrm{when} \mathrm{it} \mathrm{goes} \mathrm{off} \mathrm{again} \mathrm{(picture} \mathrm{c).} \mathrm{The} \mathrm{fluorescence} \mathrm{can} \mathrm{be} \mathrm{normalized} \mathrm{versus}$ the background and plotted considering the background as " 1 " (picture $\mathrm{d}$ ).

\subsubsection{Transcardial perfusion and processing on mice brains}

One of the most common methods for tissue fixation and subsequent protein visualization by immunohistochemistry in tissues in general and in brains in our case is the transcardial perfusion using as a fixative agent $4 \%$ paraformaldeyde in phosphate buffer solution (PFA, see solutions). This method takes advantage of the circulatory system of the animal to wash and then deliver the fixative solution throughout the body tissues, with optimal penetration in the brain. The PFA binds to the protein and, by cross-linking them, maintains the ultra-structures inside the cells allowing the antibody to bind to the antigen and preserving the fluorophores of reporter proteins such EGFP or DsRed.

Animals were sacrificed by $\mathrm{CO}_{2}$ inhalation and immediately fixed in a rack using needles. The abdominal cavity was opened and the diaphragm and the ribs were cut to allow the access to the thorax cavity.

A $0,30 \times 12 \mathrm{~mm} 30 \mathrm{G} \times 1 / 2$ needle was placed at the end of a tube connected to a peristaltic pump and was inserted inside the left ventricle while the right atrium was cut to allow the blood to leave the body. Approximately $20 \mathrm{ml}$ of ice-cold, paper-filtered, PBS pH 7.4 was perfused at a speed of 3.6 $\mathrm{ml} / \mathrm{min}$ to clean the body from the blood and then, at the same flow rate, about 50-60 $\mathrm{ml}$ of PFA were perfused. A good indicator of the quality of the perfusion is the rigidity of the tail.

The brain was then carefully removed from the skull and incubated over-night in a $15 \mathrm{ml}$ falcon tube at $4^{\circ} \mathrm{C}$ in $4 \%$ PFA in PBS for post-fixation; PFA was then substituted by $30 \%$ sucrose in PBS and the brain was left for additional 3-4 days (until the brain sinks in the solution) at $4^{\circ} \mathrm{C}$. The brain was then dried, cleaned of bones or other contaminants put in a new $15 \mathrm{ml}$ falcon tube and stored at $-80^{\circ} \mathrm{C}$.

Brains were then embedded in a cryomatrix and, using a LEICA cryostat (Leica CM 3050 S), $25 \mu \mathrm{m}$ thick coronary sections were cut and directly collected on SuperFrost ${ }^{\circledR}$ Plus microscope slides (Menzel-Glaser, Germany) and stored at $-20^{\circ} \mathrm{C}$; the cutting procedure started approximately $2 \mathrm{~mm}$ before the points of injection and ended approximately $2 \mathrm{~mm}$ after. The slides were then ready for immunohistochemistry or DAPI staining (see later).

\subsubsection{Stereotaxic injection of AAV vectors into rat striatum}

Adult female Wistar rats between 220 and $280 \mathrm{~g}$ were used for the experiments. Animals were anesthetized with a mixture of ketamine $(100 \mathrm{mg} / \mathrm{kg})$ and Xilazin $(5 \mathrm{mg} / \mathrm{kg})$. Once anesthetized, they were shaved and fixed by ear bars and a jaw holder in a stereotaxic frame (kopf instrument). The eyes were protected with bephantene and the head was cleaned and disinfected with $70 \% \mathrm{EtOH}$ and braunol. Using a scalpel a longitudinal cut was made in the middle of the head between the imaginary line connecting the eyes and the imaginary line connecting the auditory channel exposing 
both the Bregma and the Lambda. The connective tissue underlying the skin was gently removed with a scalpel, blood capillaries were cauterized and the skull was cleaned with PBS and cotton buds. Using the microinjector with a glass capillary filled with mineral oil in the top, a small bubble of air in the middle and 1-2 $\mu$ l of PBS in the bottom, the level of bregma and lambda were measured and the skull was set flat by putting the two points at the same height.

Bregma was taken as a "zero" and the coordinates for the injections were calculated using the Praxinos and Watson atlas. Once marked the point (or points) of injection in the skull, a small hole was drilled around it using a micro driller with a $0.8 \mathrm{~mm}$ tip; in this step is important not to damage the dura mater to avoid excess of bleeding. The piece of bone was then removed and the surface of the brain was rinsed with PBS.

Using a nanoliter 2000 injector coupled with a micro4 smart controller to better monitor the injection $2 \mu \mathrm{l}$ of the solution containing the viruses in PBS or the 6 hydroxydopamine (6-OHDA; see later) were injected at the desired coordinates with a flow of $500 \mathrm{nl} / \mathrm{min}$. The glass capillary was left in place for 4 minutes both before the injection, to allow the tissue to adapt to the capillary, and after injection to minimize the reflux.

The capillary was then retracted, the surface of the brain and the skull rinsed again with PBS and the skin was surgically sewn. To minimize pain $10 \mathrm{mg} / \mathrm{kg}$ of $20 \mathrm{mg} / \mathrm{ml}$ rymadil were injected subcutaneously. Animals were kept in individual cages for 5 days and then put in groups of 4-6 animals/cage.

Two sets of coordinates were used depending on the experiment; in the experiment for testing the system with EGFP as a reporter gene the viruses were injected in the middle of the left striatum and the coordinates were (in $\mathrm{cm}$ referred to Bregma): anterior +0.12 , ventral between -0.5 and -0.6 (depending on the weight of the animal) and lateral +0.25 .

To provoke the partial lesion of the dopaminergic system two deposits of 6-OHDA per animal were performed at the following coordinates (in $\mathrm{cm}$ referred to Bregma); 1 ) anterior +0.05 , lateral +0.21 , and ventral between -0.5 and -0.6 (depending on the weight), and 2 ) anterior -0.05 , lateral +0.38 , ventral between -0.5 and -0.6 (depending on the weight). Each injection consisted of $2 \mu \mathrm{l}$ of $2.5 \mu \mathrm{g} / \mu \mathrm{l}$ of 6-OHDA dissolved in saline solution $0.2 \%$ ascorbic acid. The viruses intended to restore the lesion were injected at the same coordinates.

To induce the pSwitch system animals received three intraperitoneal injections of mifepristone in three consecutive days; in case of EGFP analysis animals were sacrificed the third day after the last injection. Mifepristone was diluted in DMSO at a defined concentration in order to obtain a volume of injection of $1 \mathrm{ml} / \mathrm{kg}$.

\subsubsection{Transcardial perfusion and processing on rats brains}

As mentioned previously for mice, to visualize fluorescent proteins and for proper IHC animals were perfused and fixed using PFA. Rats were sacrificed by $\mathrm{CO}_{2}$ inhalation and were immediately fixed in a rack. The abdominal cavity was opened and the diaphragm and ribs were cut in order to expose the heart. Holding the heart steadily, a small cut was made and a blunt-ended catheter inserted through the left ventricle and atrium into the aorta. The catheter was then secured in position by clamping. Two to three hundred $\mathrm{ml}$ of ice-cold, paper-filtered, PBS pH 7.4 were pumped with a flow rate of 18 $\mathrm{ml} / \mathrm{min}$. At the beginning of the perfusion the liver was multiply cut to allow a large part of the blood 
to leave the body. After approximately a minute the abdominal aorta was clamped to restrict the perfusion to the upper part of the body and the nose was cut to give an alternative way out for the blood and the PBS.

Once the PBS flowing from the nose didn't show any trace of blood approximately $250 \mathrm{ml}$ of ice-cold 4\% PFA in PBS were infused. The perfusion efficiency can be tested by checking the rigidity of the upper part of the body.

Once the animal was perfused the head was removed and the brain was carefully removed from the skull and left in a $50 \mathrm{ml}$ tube filled with $4 \%$ PFA in PBS over night at $4^{\circ} \mathrm{C}$ for post fixation. The PFA was then substituted with $30 \%$ sucrose in PBS and the brain was left for additional $4-5$ days at $4^{\circ} \mathrm{C}$ until it sank in the solution. The brain was then dried, cleaned of pieces of bones or debris and stored at $80^{\circ} \mathrm{C}$

For sectioning, brains were embedded in a cryomatrix and, using a LEICA cryostat, $25 \mu \mathrm{m}$ thick coronary section were cut; depending on the experiment, sections were directly mounted on SuperFrost ${ }^{\oplus} \mathrm{Plus}$ microscope slides and stored at $-20^{\circ} \mathrm{C}$ or collected as a free floating sections in PBS $0,2 \%$ sodium azid and stored at $4^{\circ} \mathrm{C}$ (free floating section are better for certain $\mathrm{IHC}$ ).

\subsubsection{Processing of brain tissue for catecholamines and GDNF quantification}

In order to measure the amount of GDNF, dopamine, DOPAC and HVA brains were not fixed via perfusion of PFA.

Animals were sacrificed by $\mathrm{CO}_{2}$ inhalation, the animal was immediately decapitated and the brain extracted. Working on a plate cooled to $-80^{\circ} \mathrm{C}$ and kept on ice, both the left and the right striatum were isolated. Both striata were divided in 2 (one part for catecholamines evaluation and one part for GDNF analysis) and stored at $-80^{\circ} \mathrm{C}$ in pre-cooled cryovials (micro tube $2 \mathrm{ml}$; SARSTEDT) for further processing. The vials were previously filled with $0.6-0.8 \mathrm{~g}$ of Precellys ceramin beads $1,4 \mathrm{~mm}$ (Peqlab) spheres, weighed in advance to have the tare and weighed again with the piece of brain inside in order to calculate the net weight of the piece.

\subsubsection{Cathecolamines analysis in rat brains}

Dopamine, Dopac and HVA content in striatum was measured by an HPLC system coupled with an electrochemical detector (chapter 3.4.6 "HPLC with cell lysates of primary cortical neurons"); to extract catecholamines from the tissue previously isolated and stored at $-80^{\circ} \mathrm{C}$ in cryovials, the sample had to be first homogenized.

Fifty $\mu \mathrm{l} / \mathrm{mg}$ tissue of $0.1 \mathrm{M}$ perchloric acid (PCA) were added to the cryovials which were then processed in a washing machine (Precellys 24, Peqlab) for 3 cycles of 45 second at max shaking rate. The tubes were then centrifuged for one minute at $10.000 \mathrm{rpm}(\sim 9300 \mathrm{~g})$ at $4^{\circ} \mathrm{C}$, the supernatant was transferred to a new tube and centrifuged again for 10 minutes at max speed at $4^{\circ} \mathrm{C}$. The supernatant was then divided in $200 \mu \mathrm{l}$ aliquots and stored at $-20^{\circ} \mathrm{C}$ until analyzed. Standards were prepared by diluting known amount $(0,0.15,0.30,1.5 \mathrm{M}$ each) of the three catecholamines in 0.1M PCA.

Similar to the samples prepared from cells, standards and samples were transferred to HPLC vials and loaded to the HPLC autosampler which was kept at $6^{\circ} \mathrm{C}$ and the HPLC software was programmed to inject $20 \mu \mathrm{l}$ of the samples to the column. The flow rate of the mobile phase (HPLC buffer) was 0.4 $\mathrm{ml} / \mathrm{min}$ and the run lasted for $22 \mathrm{~min}$. The guard cell was held at $600 \mathrm{mV}$ while the first channel of 
the analytical was held at $50 \mathrm{mV}$ and the second at $400 \mathrm{mV}$. To minimize errors, standards were run every 12-15 samples and averaged; the amount of the 3 compounds was calculated by integrating the areas under the peaks. The retention time for DOPAC was around $4.8 \mathrm{~min}$, for Dopamine $5.7 \mathrm{~min}$ and for HVA $10.8 \mathrm{~min}$.

\subsubsection{GDNF measurement by ELISA}

To measure the amount of GDNF in the striatum an Enzyme-linked immunosorbent assay (ELISA) was performed. ELISA is a quantitative test based on the binding of specific antibodies to the target antigen. The amount of antigen present in solution is then evaluated by an enzymatic reaction performed by an enzyme linked to the last antibody used. With the reaction rate proportional to the amount of antigen present in solution, it is possible to evaluate the amount of GDNF in our samples of interest through comparison to a calibration curve.

Samples from rat brains were added to $300-400 \mu \mathrm{l}$ 0.1M PCA and were then processed in a washing machine with 3 cycles of 45 seconds at max shaking rate. Tubes were then centrifuged for one minute at $10000 \mathrm{rpm}$ at $4^{\circ} \mathrm{C}$, the supernatant was then transferred to a new tube and centrifuged again for 10 minutes at max speed at $4^{\circ} \mathrm{C}$. The supernatant was then divided in $100 \mu \mathrm{l}$ aliquots and stored at $-20^{\circ} \mathrm{C}$ until analyzed.

The ELISA was performed following the manufacture's instruction except for the acid treatment of the samples which was avoided. Briefly, 96-wells/plates were coated with Anti-GDNF mAb and blocked; samples were then loaded in the plates together with known samples to design a standard curve. After a washing step, Anti-Human GDNF pAb was added and the plate was incubated over night. Samples underwent another cycle of washing before incubation with Anti-Chicken IgY HRP conjugated antibody; after a last washing step the TMB One Solution was added and the colorimetric reaction was stopped after $15 \mathrm{~min}$ by adding $1 \mathrm{~N}$ hydrochloric acid. The absorbance at $450 \mathrm{~nm}$ was recorded using a microplate reader (Tecan RainBow), a standard curve was plotted, and the GDNF concentration of the unknown samples was extrapolated.

\subsubsection{Fluorescence microscopy}

\subsubsection{Preparation of mounted brain slices}

In order to evaluate EGFP or DsRed expression, brain sections were mounted directly on microscope slides and were stored at $-20^{\circ}$. Slides were dried by incubating them for $30 \mathrm{~min}$ at $37^{\circ} \mathrm{C}$ and rehydrated by placing them for few minutes in PBS. Next, slides were incubated for 10 minutes in DAPI (1:50.000 in PBS) and washed 3 times with PBS. Cover slips were then mounted to the slides using mowiol and left to dry over night at $4^{\circ} \mathrm{C}$.

Brain section were then be imaged using a Zeiss Axioplan 2 microscope equipped with a CCD camera and axiovision rel 4.7 software. Images were then optimized using Adobe Photoshop 7.0.

\subsubsection{Immunohistochemistry (IHC) on free floating brain slices}

When performing $\mathrm{IHC}$, brain sections were collected as free floating slices which were stored at $4^{\circ} \mathrm{C}$ in PBS $0.1 \%$ sodium azid.

Brain slices were transferred in 12 wells/plates (4-5 slices/well) and washed three times with PBS to eliminate the sodium azid. Slices were then incubated in TBS pH 9 at $60^{\circ} \mathrm{C}$ for different amounts of time (see table) for antigen retrieval (AR). After three 5 minutes washing steps with PBS slices were incubated with 5\% NGS $0.1 \%$ Triton in PBS for blocking. Slices were then incubated with the first 
antibody diluted in PBS 2\% NGS 0.1\% Triton. Antibody dilution and incubation conditions varied depending on the antigen (table 6). After the washing steps with PBS ( $3 \times 5 \mathrm{~min}$ ), the slices were incubated for 1 hour at $37^{\circ} \mathrm{C}$ with the secondary antibody diluted in PBS 2\% NGS 0.1\% Triton (for dilutions see table). Another three 5 minute washing steps were performed before incubating slices in DAPI (1:50.000 in PBS) for 10 minutes at RT. Slices were washed again three times in PBS and mounted in superfrost microscope slides (Menzel-Glaser, Germany). Slices were left to dry for 30 minutes, rehydrated by immersing them in PBS for 1 minute, covered with a cover slips and mounted using mowiol. Slides were left to dry over-night at $4^{\circ} \mathrm{C}$ and were then imaged with a Zeiss Axioplan 2 microscope as previously mentioned.

\begin{tabular}{|c|c|c|c|c|c|c|}
\hline Antigen & species & dilution & $\begin{array}{l}\text { Incubation } \\
\text { time }\end{array}$ & $\begin{array}{c}\text { AR } \\
\text { time }\end{array}$ & Company & $\begin{array}{c}\text { Ord. } \\
\text { number }\end{array}$ \\
\hline \multicolumn{7}{|l|}{ Primary antibody } \\
\hline Anti Iba1 & $\begin{array}{c}\text { Rabbit } \\
\text { polyclonal }\end{array}$ & $1: 200$ & $\begin{array}{l}\text { o/n @ RT + } \\
\text { shake }\end{array}$ & $2 \mathrm{~h}$ & $\begin{array}{l}\text { Waxo } \\
\text { Chem }\end{array}$ & $\begin{array}{c}019 \\
19741\end{array}$ \\
\hline $\begin{array}{l}\text { Anti GFAP (for } \\
\text { immunological reaction) }\end{array}$ & $\begin{array}{c}\text { Rabbit } \\
\text { polyclonal }\end{array}$ & $1: 600$ & $\begin{array}{c}\text { o/n @ RT + } \\
\text { shake }\end{array}$ & No $A R$ & DAKO & Z0334 \\
\hline $\begin{array}{l}\text { Anti GFAP ( for cell type } \\
\text { identification) }\end{array}$ & $\begin{array}{c}\text { Rabbit } \\
\text { polyclonal }\end{array}$ & $1: 200$ & $\begin{array}{l}\text { o/n @ RT + } \\
\text { shake }\end{array}$ & No $A R$ & DAKO & Z0334 \\
\hline Anti NeuN & $\begin{array}{c}\text { Mouse } \\
\text { monoclonal }\end{array}$ & $1: 200$ & $\begin{array}{c}\text { o/n @ RT + } \\
\text { shake }\end{array}$ & $6 \mathrm{~h}$ & $\begin{array}{l}\text { Chemicon } \\
\text { Millipore }\end{array}$ & ab 377 \\
\hline Anti TH & $\begin{array}{c}\text { Rabbit } \\
\text { polyclonal }\end{array}$ & $1: 1000$ & $\begin{array}{c}3 \text { days @ } \\
4^{\circ} \mathrm{C} \\
\end{array}$ & No AR & $\begin{array}{l}\text { Chemicon } \\
\text { Millipore }\end{array}$ & ab 152 \\
\hline Anti S100 & $\begin{array}{c}\text { Rabbit } \\
\text { polyclonal }\end{array}$ & $1: 500$ & $\mathrm{o} / \mathrm{n} @ 4^{\circ} \mathrm{C}$ & $2 \mathrm{~h}$ & DAKO & Z0311 \\
\hline \multicolumn{7}{|l|}{ Secondary antibody } \\
\hline Cy 3 anti rabbit & Goat & $1: 250$ & $1 \mathrm{~h} @ 37^{\circ} \mathrm{C}$ & I & Dianova & $\begin{array}{c}111- \\
165-006\end{array}$ \\
\hline Cy 3 anti mouse & Goat & $1: 250$ & $1 \mathrm{~h} @ 37^{\circ} \mathrm{C}$ & / & Dianova & $\begin{array}{c}115- \\
165-003\end{array}$ \\
\hline
\end{tabular}

Table 6) List of antibodies used for IHC. Iba1 = microglia specific; GFAP = astrocyte specitic; NeuN = neuronal specific; TH = tyrosine hydroxylase (DA neurons); $\mathbf{S 1 0 0}$ = astrocyte specific; $\mathbf{A R}=$ antigen retrieval; $\mathbf{R T}=$ room temperature; $0 / \mathrm{n}=$ over night.

\subsubsection{Motor functional tests}

Animals with a unilateral lesion of the dopaminergic system show some deficits in the motor skills that can be detected by appropriate motor tests. The detected deficits should be proportional to the size of the lesion.

\subsubsection{Apomorphine induced rotation}

The injection of 6-OHDA in the striatum causes a selective loss of dopaminergic neurons; to compensate the lack of dopamine the target neurons overexpress the dopamine receptors becoming in this way hypersensitive. The presence of the dopamine agonist apomorphine, hyperstimulates the target neurons and induce the animal to rotate contralateral to the lesion. The number of $360^{\circ}$ total rotation should be proportional to the size of the lesion.

Apomorphine was diluted in saline solution at $0.5 \mathrm{mg} / \mathrm{ml}$ concentration and $1 \mathrm{ml} / \mathrm{kg}$ was injected intraperitoneally to the rats. Animals were then placed in a $20 \times 30 \mathrm{~cm}$ (height $x$ diameter) cylinder 
which was covered with a transparent lid. Animals were recorded for one hour with a digital camera (Hercules ${ }^{\circledR}$ ) connected to a computer with the Webcam Station Evolution (SE) software (Hercules ${ }^{\circledR}$ ). The number of total turns was counted in a second moment using VLC mediaplayer software and results are here presented as turns /hour.

\subsubsection{Cylinder test}

The cylinder test takes advantage of the natural curiosity of rats that pushes them to explore the environment around them. Once the animal is placed in a cylinder it will start to explore the new environment by climbing the wall its front paws. By counting the number of times in which the animal put his weight only on the left paw, only on the right one or in both we can estimate if an unbalance of dopamine is present in the brain. A healthy rat should use around $20 \%$ only the left paw, around $20 \%$ only the right and around $60 \%$ both of them together. If the animal has a lesion in the left striatum (as in our case) the left paw, controlled by the right hemisphere will be preferred.

In our experiment animals were placed in a transparent cylinder 30x17 cm (height $\mathrm{x}$ diameter) for 10 minutes and we recorded them with a Hercules ${ }^{\circledR}$ digital camera and the SE software. To help the visualization when the animal's back was to the camera, two mirrors were placed behind the cylinder so that the whole perimeter of the cylinder could be imaged.

The videos were then analyzed using the VLC software and the data are here showed as left/total touches $* 100$.

\subsubsection{Corridor test}

It was demonstrated [109] that a 6-OHDA lesion in the striatum affects the olfactory skills of the animal. To test this sense the animal is put in a narrow corridor with small dishes in both sides filled with food. Animals without olfactory deficit will smell the food from both sides and it will feed from the left side $50 \%$ of the time and from the right $50 \%$ of the time. If the animal has a lesion that affect the olfactory perception, it may smell and feed preferentially from one of the dishes.

In our experiments a 2 meter long, $7 \mathrm{~cm}$ wide and $10 \mathrm{~cm}$ height corridor with transparent lateral walls and lid was built. two series of 14 dishes $0.5 \mathrm{~cm}$ height and with a diameter of $1 \mathrm{~cm}$ were placed at the two sides of the corridor with a $14 \mathrm{~cm}$ distance between one and the next one. The dishes were filled with a few mg of crumbled corn Flakes. Rats were trained to the new environment by placing them in three consecutive days in the corridor for 10 minutes with crumbled corn Flakes spread everywhere. The procedure was repeated once the day before each subsequent test. Before the tests animals were starved for 6-8 hours. The test lasted until the animal had completed 20 food withdrawals from the dishes. Results are presented as left/total withdrawals * 100.

\subsection{Lineout of the experiments}

\subsection{1 pSwitch system}

The first part of the project consisted of optimizing the viral vectors in order to obtain an acceptable compromise between the off- and the on-states. The pSwitch system was transferred from the invitrogen vectors to AAV vectors suitable for virus production and the EGFP was used as a reporter gene to evaluate the two states of the system. All the viruses were first designed in silicio, prepared, tested in cell culture when possible (AAV5 does not infect primary cortical neurons in culture), and injected in vivo stereotaxically in the rat striatum. Depending on the results, different variables were evaluated: 1) the insertion/omission of the transcription elements WPRE and TBs, 2) effect of 
different polyadenylation sequences, 3 ) the combination of the two cassettes in only one vector with different configurations, 4) the use of different promoters for the pSwitch protein and 5) the packaging of the vectors in different serotypes.

Once obtained the right compromise between the on- and the off-states, a large court of animals was injected with the chosen viruses and different groups of animals were sacrificed at different time points in order to study the course of the fluorescence over different cycles of activation (figure 8).

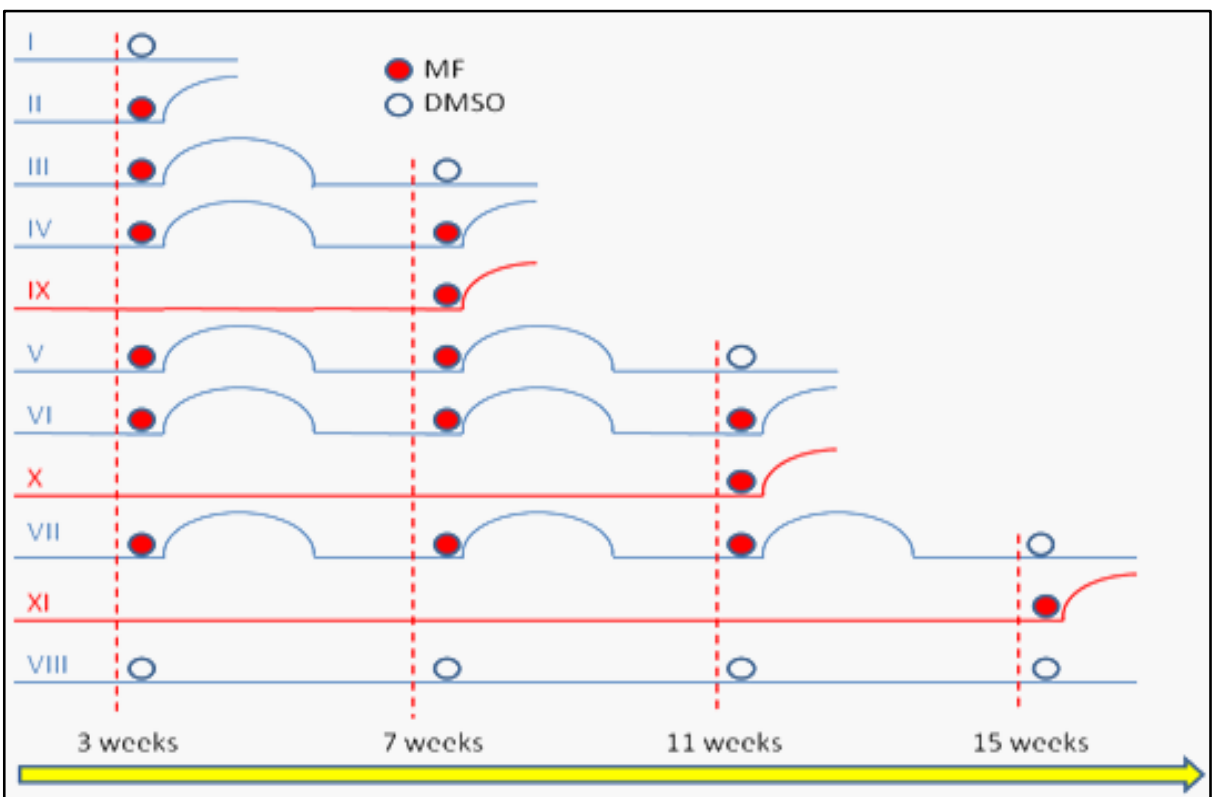

Figure 8) Time table of multiple induction experiment. Different group of animals were injected with mifepristone (red dots) or with DMSO (white dots) and sacrificed at different time points. The ends of the blue lines represent the time point when animal were sacrificed; if the line is curved the system was supposed to be active while with the flat line we expect no EGFP expression. Red lines refer to control groups. MF = mifepristone.

To make most efficient use of animals and study the behavior of the system in vivo some combination of viruses were injected in mouse cortex, a cranial window was put over the injection point, and the course of the fluorescence was followed using a 2-photons microscope or an OV-100 microscope.

Parallel experiments were performed to study the dosage of the inducing drug mifepristone, to study the use of different promoters and to study the interactions between different viruses.

Simultaneously to these experiments a rat model of PD was generated; for this purpose different amounts of 6-OHDA (a drug which selectively kills only dopaminergic neurons) were stereotaxically injected at different coordinates in the rat striatum with the aim to achieve a 50-60\% loss of the dopaminergic innervations in the striatum. This experiment was performed by Dr. Yuliya Tereschenko. 


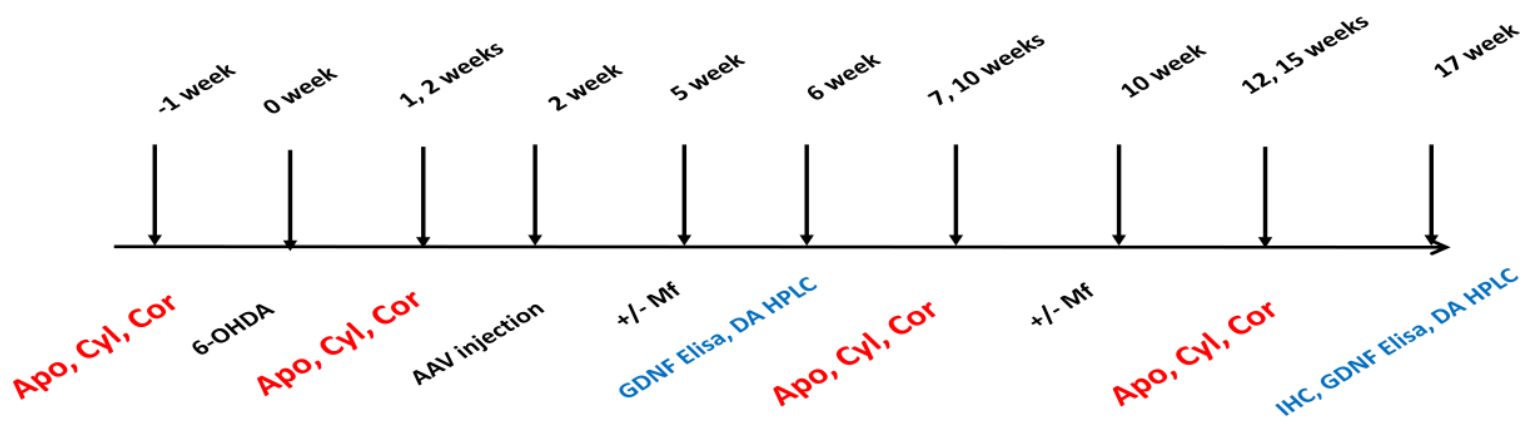

Figure 9) Time-table of the 6-OHDA lesion and restoration experiment. Animals were tested for the motor tests before the operation (week -1). Rats were then injected with 6-OHDA (week 0), tested again (week 1-2) and injected with the 4 different combinations of viruses (week 2). Half of the animals which received the pSwitch system were injected with MF at week 5 and 10 while the other half received DMSO. Rats were tested for the three motor tests at week 7, 10, 12 and 15. Some of the animals in each group were sacrificed at week 6 and 17 in order to measure GDNF level by ELISA and Catecholamines concentration by HPLC. Leftover animals were sacrificed and perfused at week 17 for histological analysis. Apo = apomorphine induced rotation; $\mathrm{Cyl}=$ cylinder test; Cor = corridor test; 6-OHDA = 6-OHDA lesion; DA = dopamine.

Once the lesion model was established and the optimal vectors characterized, the EGFP gene was substituted with the gene of the neurotrophic factor GDNF and animals received first the lesion and then the viruses. To follow the degeneration/regeneration of the dopaminergic system different motor tests, biochemical tests and histological tests were performed (figure 9).

In this experiment we compared the expression of GDNF in neurons (performed myself) and in astrocytes (experiment performed by Dr. Yuliya Tereshchenko).

\subsubsection{Dopamine producing neurons}

As DA neurons account only for a small fraction of the total number of neurons in the rat brain, it is not possible to obtain primary culture with more than $10 \%$ of DA neurons [110]. In order to study the interaction between DA and $\alpha$-synuclein primary cortical neurons in culture were transduced with different combination of viruses expressing the enzymes necessary for DA production. These enzymes consist of: TH, AADC, GCH1, and VMAT2.

Genes were purchased from Imagenes and transferred from shuttle plasmids to AAV vectors suitable for viral vector production. Protein expression was then confirmed by IHC and western blots.

The first part of the project consisted in the use of different combination of vectors and substrates for dopamine production in order to obtain an easy to handle reproducible system. The amount of dopamine and its metabolites DOPAC and HVA was measured using a HPLC system coupled with an electrochemical detector.

Once achieved the dopamine production, a study on the interaction between $\alpha$-synuclein and dopamine was performed. Experiments include measurements of DA production by HPLC, viability tests, and western blots to evaluate aggregation properties of $\alpha$-syn. 


\section{Results}

\subsection{Summary}

During this thesis two projects were carried out: the main one consisted of a study of an AAV-based gene regulatable system for neurotrophic factor delivery in the CNS and a second one aiming to generate dopaminergic-producing neurons from primary cortical neuron in order to assess eventual interaction between dopamine and $\alpha$-syn.

In the first part of the main project, by using EGFP as a reporter protein, it is shown that by starting from a combination of vectors with high background expression in the off-state and, by studying the role of isolating elements (TB), transcription control elements (WPRE) and different AAV serotypes, we were progressively able to decrease the background expression of the off-state to a level considered acceptable. Once established the gene regulatable system, reiterate activations, inducer MF dosage, and immunological response in the rat brain due to the system were studied. In a following step, EGFP was substituted with GDNF, a 6-OHDA rat model of PD was generated and the effect of short pulses of expression of the NF was studied in order to evaluate a possible restorative effect.

In the second project different approaches were pursued in order to obtain dopamine producing neurons by transducing primary cortical neurons with AAV's expressing genes essential for catecholamine synthesis. Once a reproducible strategy had been identified, the interaction between dopamine and $\alpha$-synuclein was evaluated showing a tendency of neurons to synthesize and release less dopamine in presence of $\alpha$-synuclein.

\subsection{Generation of a low background regulated transgene expression system}

Different constructs were cloned, tested and modified depending on the results; the aim was to achieve a zero level of transgene expression in the off-state and an acceptable level of expression in the on-state.

Two different serotypes have been used: AAV5 and AAV6, which show different transduction properties when used in rat striatum; serotype 5 is known to spread more and deliver less viral genomes per cell in the striatum comparing to serotype 6 [111].

Two different concepts have been followed to adapt the pSwitch system to the AAV vectors: a two vector system in which the two cassettes were cloned and packed in two separate vectors and a one vector system where the two cassettes where cloned in a single vector. The advantage in adopting the two vectors consists in having the possibility to vary the ratio between the two cassettes while the use of one vector simplifies the system (figure 10).

The first group of experiments was performed using viruses packed in the AAV6 serotype while in the second group AAV5 serotype was used (in both cases the single and the double vectors system were tested). The choice of starting experiments with AAV6 was due to two advantages of this serotype over AAV5: AAV6 production is faster and more efficient compared to AAV5 and in contrast to serotype 5, AAV6 transduces neuronal culture with high efficiency. These two properties allowed a fast screening of different constructs. Once prepared, AAV6 viruses were first tested in primary cortical neurons in culture. The basic protocol consisted of transducing neurons in 24 wells/plates on DIV2 (day in vitro 2), inducing EGFP expression with $10 \mathrm{nM}$ total concentration of MF on DIV5 and acquiring the pictures of neurons on DIV9. Transduction of primary cortical neurons in culture has 
been skipped when AAV5 was used as this serotype is not suitable for efficient transduction of primary neurons in culture.

All viruses were essayed in vivo in the striatum of female Wistar rats; for the stereotaxic injections viruses were diluted in $2 \mu \mathrm{l}$ of PBS and the coordinates of injection were always the same (in $\mathrm{mm}$

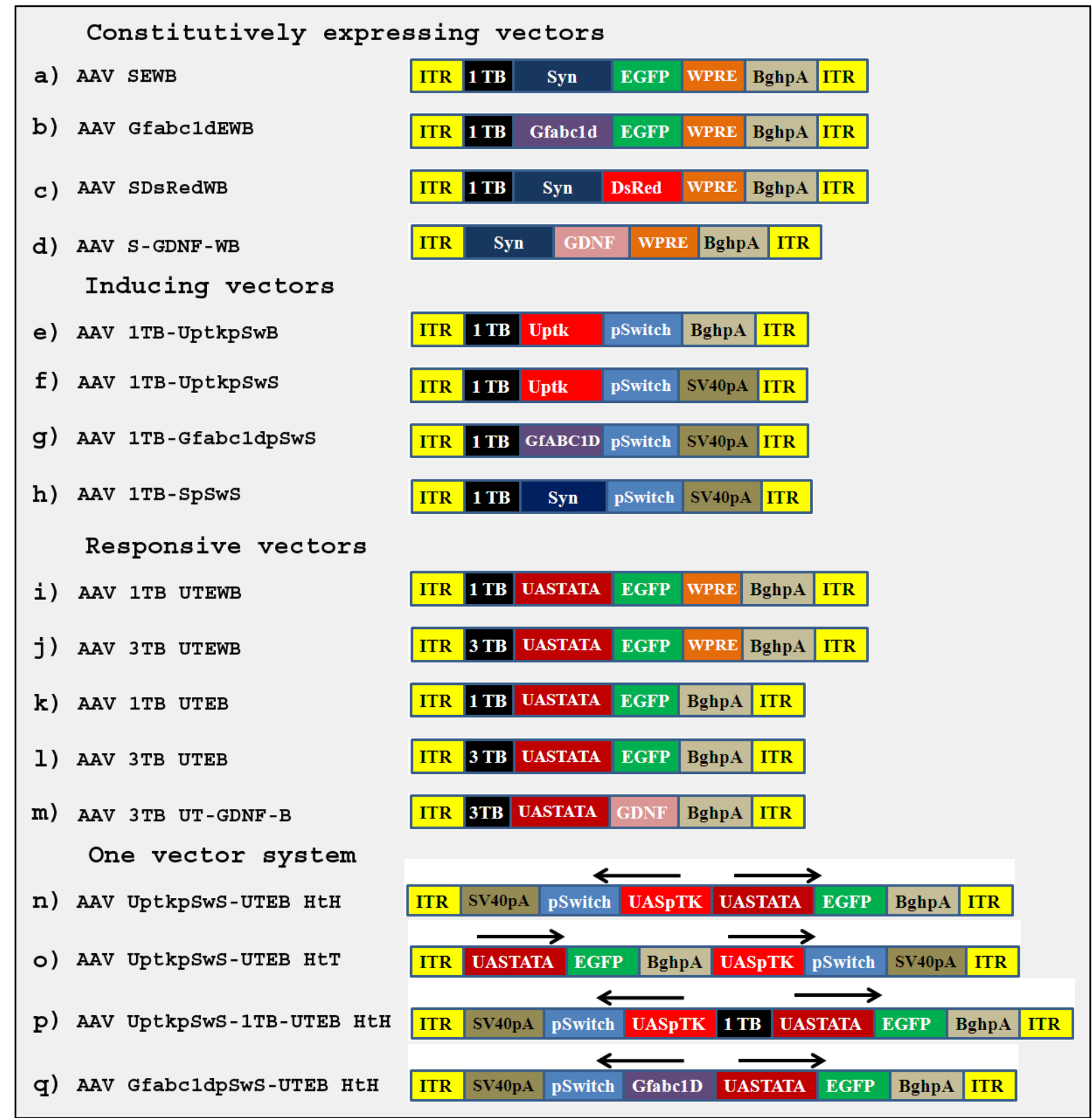

Figure 10) Representation of the main used plasmids: AAV = adeno associated virus; ITR = inverted terminal repeat; TB = transcription blocker; Syn = S = synapsin promoter; EGFP = enhanced green fluorescence protein; WPRE = Woodchuck Hepatitis Virus (WHP) Posttranscriptional Regulatory Element; Gfabc1d = astrocytic promoter; BghpA = B = bovine growth hormone poli adenylation sequence; DsRed = red fluorescent protein; GDNF = glial cell derived neurotrophic factor; Uptk = Uptk = UAS (Upstream activation sequence) Herpes Simplex Virus thymidine kinase (TK) minimal promoter; $p S w i t c h=p S w=p S w i t c h$ protein; SV40pA = S = Simian virus 40 poli adenylation sequence; $\mathrm{HtH}=$ head to head; $\mathrm{HtT}=$ head to tail. 
referring to Bregma): anterior $+1,2$, lateral $+2,5$ and ventral between 5 and 6 . The MF induction took place 2 to 3 weeks post surgery and consisted of one intraperitoneal injection/day of the steroid diluted on DMSO for three consecutive days. Animals were perfused the third day after the last MF injection, brains were cut and brain slices were mounted on cover slips for DAPI staining. Each group mentioned in the following chapters consisted of two to five animals and only a representative picture of each group is shown here. Due to the large amount of animals and constructs, evaluation of EGFP expression in the off- and in the on-states was performed only qualitatively by observing brain sections at the microscope. Brief comments to explain the reason for the following steps are added.

\subsubsection{Two vector system in AAV6}

The use of a two vector system allowed us to administrate the two cassettes in a 3:1 (responsive:inducing) ratio as supported by the description of the system (invitrogen). With the aim of studying the effect of transcription blockers (TB) to isolate the inducible promoter from ITR promoter activity [112], and of the transcription enhancer element WPRE, four different combinations of vectors were tested.

The pSwitch ${ }^{\mathrm{TM}}$ system was purchased from Invitrogen ${ }^{\mathrm{TM}}$ and the two cassettes were delivered in two separate vector (pSwitch and pGene/V5-his).In the first experiment the two cassettes were transferred from these two vectors to two different AAV vectors. In the inducing vector the ubiquitous promoter Uptk was kept and, in the responsive vector, the EGFP DNA sequence was inserted to serve as a reporter protein; the enhancing transcription sequence WPRE was added and one transcription blocker (TB) was placed between the ITR and the UASTATA promoter. The two viruses were called AAV6 UptkpSw-B (figure 10-e) and AAV6 1TB-UTEWB (figure 10-i).

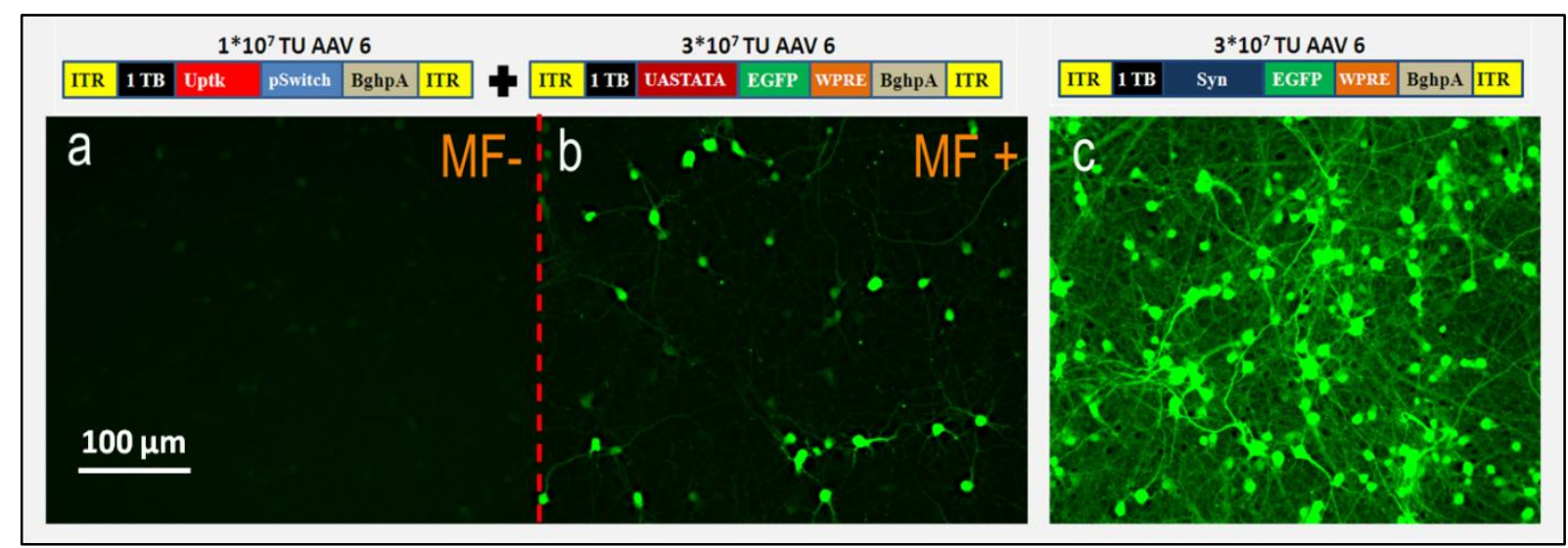

Figure 11) Cortical neurons infected with $3^{*} 10^{7}$ TU/well of the responsive vectors AAV6 1TB UTEWB $+1 * 10^{7}$ TU/well of the inducing vector AAV6 1TB UptkpSWB (pictures a and b) and the positive control AAV6 SEWB $3 * 10^{7}$ TU/well (picture c). MF induction can be observed by comparing picture a versus b. Neurons infected with AAV6 SEWB (virus expressing EGFP from synapsin promoter constitutively show a much higher level of EGFP expression (picture a versus c). Exp time $=1000 \mathrm{~ms}$

In order to further isolate the UAS TATA promoter from the promoter activity given by ITRs, 2 additional TBs were cloned between the ITR and the UAS TATA promoter; In addition the BgHpA in the inducing plasmid was exchanged with the SV4OpA sequence; this was done to prevent possible recombinations of the two vectors after cell transduction. The two vectors were called AAV6 UptkpSwS (figure 10-f) and AAV6 3TB-UTEWB (figure 10-j). 
To study the WPRE effect on EGFP expression level two new responsive vectors lacking the WPRE sequence were prepared, named AAV6-1TB-UTEB (figure 10-k) and AAV6-3TB-UTEB (figure 10-I) and used in combination with AAV6 Uptk-pSwS (figure 10-f).

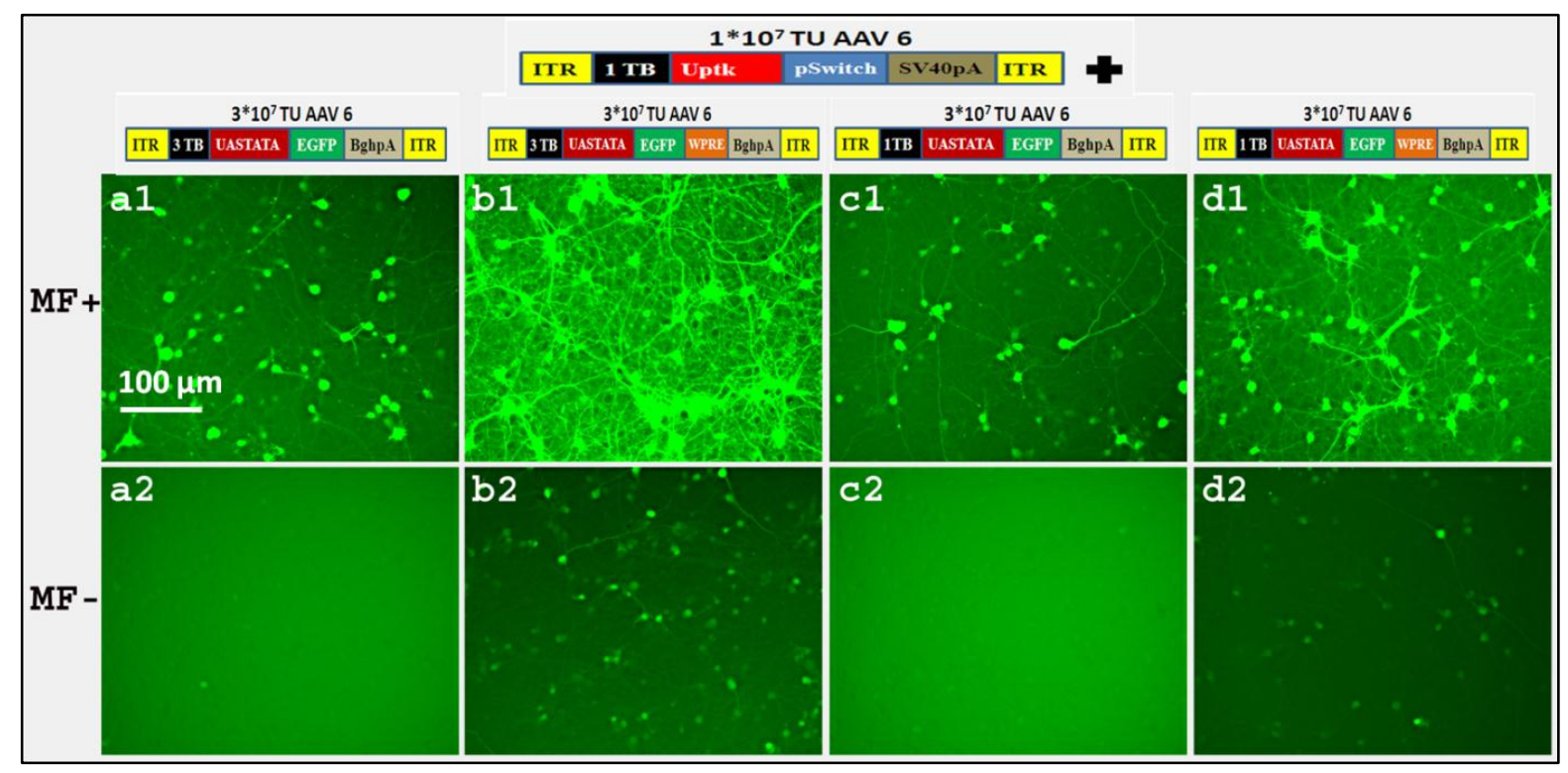

Figure 12) WPRE effect in primary cortical neurons. All samples were transduced with $1 * 10^{7}$ TU/well of AAV6-UptkpSWS + 3*107: AAV6 3TBUTEB (a), 3TB UTEWB (b), 1TB UTEB (c) and 1TB UTEWB (d).Neurons in upper panel were induced with MF while neurons in power panel were not. By comparing picture a versus $b$ and picture $c$ versus $d$ the WPRE effect in increasing EGFP expression is evident both in induced and not induced conditions. Acquisition time = $8500 \mathrm{~ms}$.

As mentioned, vectors were first tested in vitro by transducing neurons in 24 wells/plates with $1 * 10^{7}$ $\mathrm{TU} /$ well of the inducing vector and $3^{*} 10^{7} \mathrm{TU} /$ well of the responsive vector. As a positive control neurons were infected with $3 * 10^{7}$ TU/well of the constitutively EGFP expressing virus AAV6 SEWB (figure 10-a).

As can be seen in figure 11 the activating effect of MF can be clearly seen by comparing induced and not induced samples (picture a versus $b$ ); in all cases EGFP expression was much lower than what observed in the positive control AAV6 SEWB. Once the system was activated, EGFP expression did not return back to the basal level of expression.

In order to highlight possible EGFP expression without MF induction, the pictures in figure 12 were acquired with an exposure time of $8500 \mathrm{~ms}$. As can be seen, WPRE increases the EGFP expression in the on-state but leads to a low but detectable expression in the off-state (picture a versus $b$ and $c$ versus d). No major difference in EGFP expression was noticed when 1 or 3 TBs were used (picture a versus $c$ and $b$ versus $d$ ).

In summary we verified that, in vitro, the presence of the enhancing transcription control element WPRE increase the EGFP expression both in the MF-induced and in the not induced state, leading to background EGFP expression in the off-state. No substantial difference was noticed when the ITR promoter activity was isolated with 1 or 3TBs. The pSwitch system was then tested in vivo by injecting the viruses stereotaxically in rat striatum. 
The first in vivo experiment was performed using AAV6 UptkpSw-B (figure 10-e) + AAV6 1TB-UTEWB (figure 10-i). The following groups of animals were prepared:

a. AAV6 -1TB-UTEWB $2.75 * 10^{8}+$ AAV6 UptkpSwB $0.9 * 10^{8}$ not induced

b. AAV6 -1TB-UTEWB $2.75 * 10^{8}+$ AAV6 UptkpSwB $0.9 * 10^{8}$ induced with MF $200 \mu \mathrm{g} / \mathrm{kg}$

c. AAV6 -1TB-UTEWB $2.75 * 10^{8}+$ AAV6 UptkpSwB $0.9 * 10^{8}$ induced with MF $20 \mathrm{mg} / \mathrm{kg}$

d. AAV6-1TB-UTEWB $2.75 * 10^{8}$ (negative control)

e. AAV6 SEWB $2.75^{*} 10^{8}$ (figure 10 -a) (positive control)

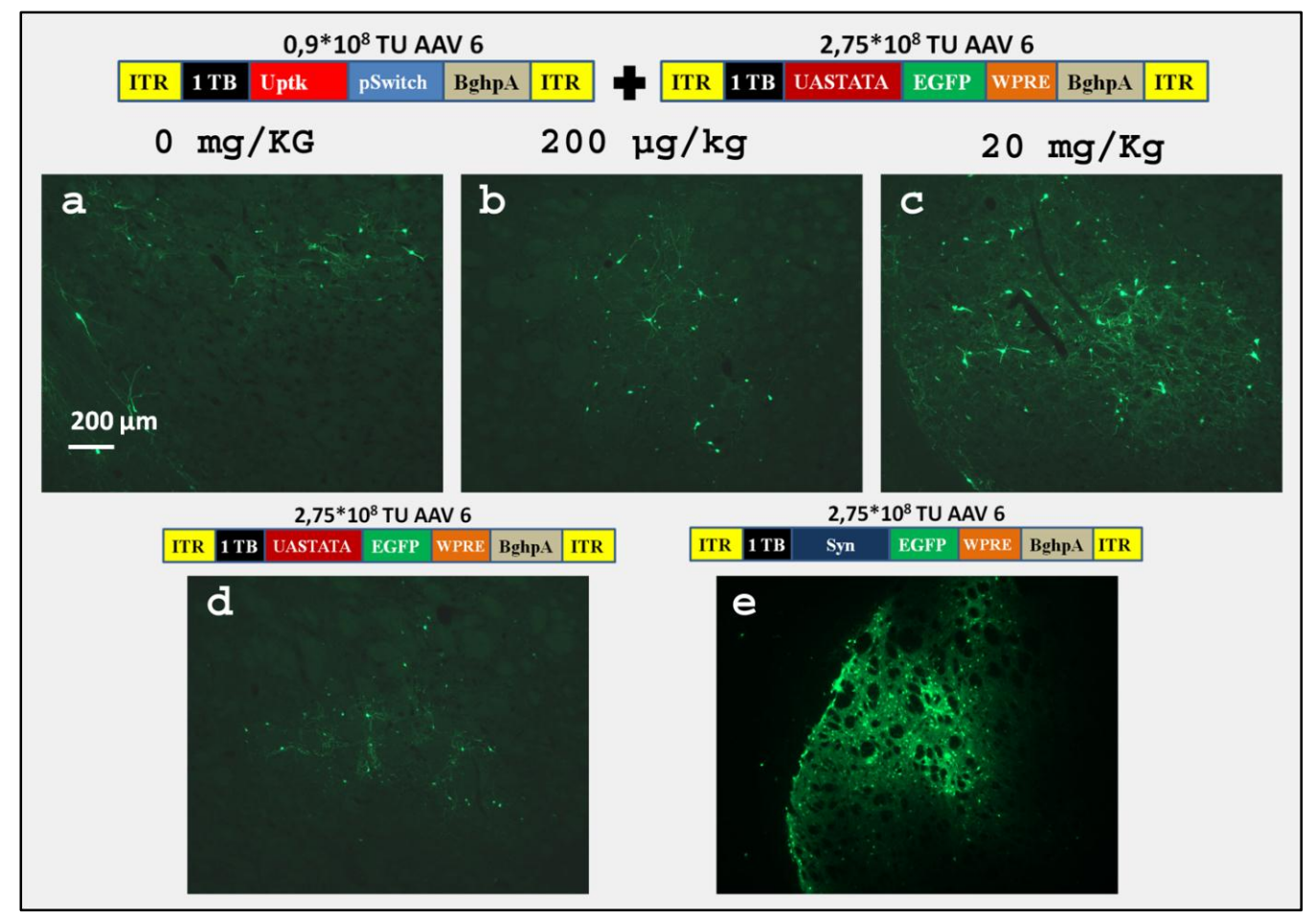

Figure 13: $25 \mu \mathrm{m}$ thick brain coronal sections of Wistar rats injected with AAV6 -1TB-UTEWB $2.75 * 10^{8}+$ AAV6 UptkpSwB $0.9 * 10^{8}$ (pictures a-c), with only AAV6 -1TB-UTEWB $2.75 * 10^{8}$ (picture d) and with AAV6 SEWB $2.75 * 10^{8}$ (picture e). No difference can be seen between animals without induction (picture a), induced with $200 \mu \mathrm{g} / \mathrm{kg}$ of MF (picture b) and injected only with the responsive vector (picture d). Animals induced with $20 \mathrm{mg} / \mathrm{kg}$ of MF seem to show a slightly higher EGFP expression (picture c). EGFP expression of animals injected with AAV6 SEWB is much higher than all the others (picture e). Acquisition time a-d = $5000 \mathrm{~ms} ; \mathrm{e}=\mathbf{6 0 0} \mathrm{ms}$.

Similar to what observed in cell culture, the EGFP expression in animals that received AAV6 SEWB (figure 13 picture e) was much higher compared to all the other groups (figure 13 pictures a-d). The expression of the reporter gene is present in all groups and the group that received $20 \mathrm{mg} / \mathrm{kg}$ of MF (figure 13 picture c) shows only a slightly higher expression of EGFP. No difference can be seen between group a (no MF induction), b (200 $\mathrm{\mu g} / \mathrm{kg}$ of MF) and d (only responsive vector) suggesting that the EGFP expression in the off-state derives from a leakage of the responsive vector promoter in absence of activation.

In conclusion, with this combination of vectors (AAV6 -1TB-UTEWB + AAV6 UptkpSwB), the EGFP expression in the off-state is substantial and we could not demonstrate both the inducibility and the dose response of the system. The EGFP expression in the not induced state might be due to the proximity of the UASTATA promoter to the ITR which are thought to act as a promoter in the wild type viruses [112]. To solve this problem 2 additional TBs were added. The lack of a strong difference between the on and off states might be due to a too low dosage of MF, thus a higher dosage was 
tested in the subsequent combination of viruses. In addition in the following experiments the BgHpA in the inducing vector was exchanged with the SV4OpA sequence; this was done to prevent possible recombinations of the two vectors after cell transduction.

The second combination of viruses tested was AAV6 UptkpSw-S (figure 10-f) + AAV6 3TB UTEWB (figure 10-j) and animals were divided into the following groups:

a. AAV6 -3TB-UTEWB $2.75 * 10^{8}+$ AAV6 UptkpSwS $0.9 * 10^{8}$ not induced

b. AAV6 -3TB-UTEWB $2.75 * 10^{8}+$ AAV6 UptkpSwS $0.9 * 10^{8}$ induced with MF $20 \mathrm{mg} / \mathrm{kg}$

c. AAV6 -3TB-UTEWB $2.75 * 10^{8}+$ AAV6 UptkpSwS $0.9 * 10^{8}$ induced with MF $50 \mathrm{mg} / \mathrm{kg}$

d. AAV6-3TB-UTEWB $2.75 * 10^{8}$ (negative control)

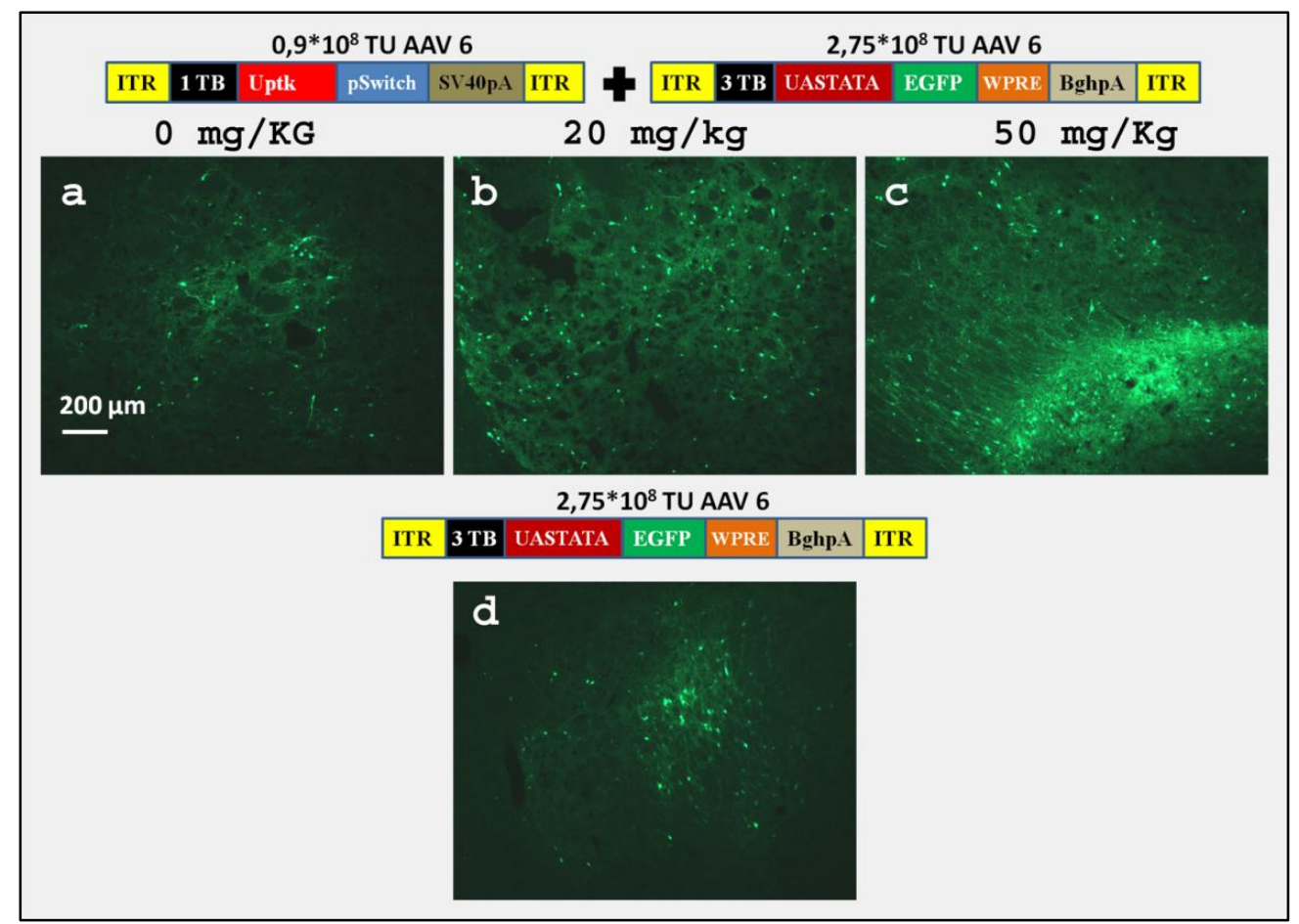

Figure 14) $25 \mu \mathrm{m}$ thick brain coronal sections of Wistar rats injected with AAV6 -3TB-UTEWB $2.75 * 10^{8}+$ AAV6 UptkpSwS $0.9 * 10^{8}$ (pictures a-c) and only with AAV6 -3TB-UTEWB $2.75 * 10^{8}$ (picture d). The activation of the system upon MF induction can be assessed by comparing animals that did not received MF (picture a) or that were injected only with the responsive vector (picture d) and animals that were induced with $20 \mathrm{mg} / \mathrm{kg}$ (picture b) and $50 \mathrm{mg} / \mathrm{kg}$ (picture c) of the steroid. Background expression is high and a dose response can be deduced by comparing the difference in EGFP expression in pictures a (not induced), b (20 mg/kg MF), and c (50 mg/kg MF).

From figure 14 pictures a and $d$, in which are shown respectively the group which did not receive the MF induction and the negative control without inducing vector, it can be seen that the 3TBs were able to decrease, but not abolish, the EGFP expression. The effect of MF is evident and can be seen in animals that received both 20 (figure 14 picture b) and $50 \mathrm{mg} / \mathrm{kg}$ (figure 14 picture c) of the drug. The system seems to be dose-responding but not enough animals were tested with the higher dosage as it resulted in obvious impairment of animal health deduced by hunched posture and lethargic behavior for one day after injection of MF. As a consequence only the $20 \mathrm{mg} / \mathrm{kg}$ dosage was used in the following experiments.

Even if decreased, the EGFP expression in the off-state was still clearly detectable and to further decrease it, the next step consisted in omitting the transcription enhancing sequence WPRE. In the previous experiments we have already demonstrated that the use of 3TBs gives better results 
compared to the use of only 1TB in respect to lower background expression in the off-state; for this reason the vector with only 1TB and without WPRE (AAV6-1TB-UTEB; figure 10-k) was not investigated in vivo and only the vector with 3TB and without WPRE AAV6 3TB-UTEB (figure 10-I) was used for stereotaxic injection in combination with the inducing vector AAV6 UptkpSwS (figure 10-f). The following three groups were prepared:

\section{a. AAV6 -3TB-UTEB $2.75 * 10^{8}+$ AAV6 UptkpSwS $0.9 * 10^{8}$ not induced with MF $0 \mathrm{mg} / \mathrm{kg}$ \\ b. AAV6 -3TB-UTEB $2.75 * 10^{8}+$ AAV6 UptkpSwS $0.9 * 10^{8}$ induced with MF $20 \mathrm{mg} / \mathrm{kg}$ \\ c. AAV6-3TB-UTEB $2.75 * 10^{8}$ (negative control)}

In figure 15 it is shown that the expression level of EGFP in animals that did not receive MF (figure 15 picture a) or that were injected only with the responsive vector (figure 15 picture c) appears to be lower than in the previous experiments (figure 14 picture d). Nevertheless EGFP positive cells are still clearly present indicating that the 3TB UAS TATA promoter is not completely silent when notinduced. The activation of the pSwitch system can be easily identified by comparing animals that did not received the inducing drug and animals which received $20 \mathrm{mg} / \mathrm{kg}$ of MF (picture a versus $\mathrm{b}$ ).

The best result was obtained by including 3TB and omitting WPRE but, despite the good induction upon MF addition, the background level of expression in the off-state is still considerably high. In the following experiments we evaluated the possibility of having both cassettes packed in a single vector, in this way obtaining a complete isolation of the inducible promoter from the ITRs.

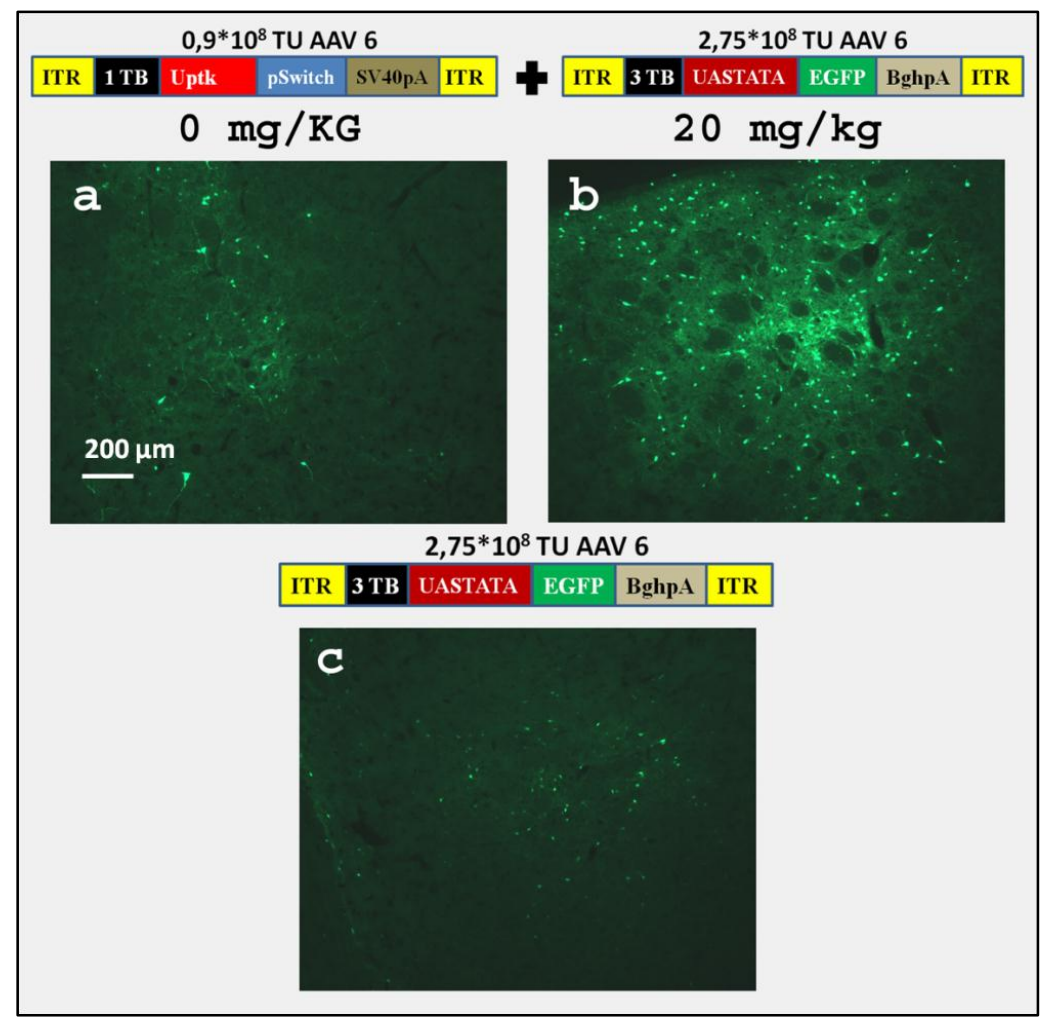

Figure 15) $25 \mu \mathrm{m}$ thick brain coronal sections of Wistar rats injected with AAV6 -3TB-UTEB $2.75 * 10^{8}+$ AAV6 UptkpSwS $0.9 * 10^{8}$ (picture $a, b$ ) and only with AAV6 -3TB-UTEWB $2.75 * 10^{8}$ (picture c). The activation of the system upon MF induction can be assessed by comparing animals that did not receive MF (picture a) and animals that were induced with $20 \mathrm{mg} / \mathrm{kg}$ (picture b). Background expression without induction remains clearly detectable. Acquisition time $=5000 \mathrm{~ms}$. 


\subsubsection{One vector system in AAV6}

In addition of a further isolation of the inducible promoters from the ITRs, the use of 1 vector instead of 2 renders the experiment more controllable. The omission of WPRE allowed us to have the two cassettes small enough to fit them together in a unique AAV vector which has a maximum packaging size of about $4.8 \mathrm{~kb}$.

Four different constructs were generated, three with the ubiquitous Uptk promoter, and one with the astrocytic Gfabc1d promoter. The two cassettes UptkpSwS and UTEB were inserted in a unique vector with two different configurations generating the AAV UptkpSwS-UTEB Head to Tail (HtT), where Head to Tail means that the two cassettes are oriented consecutively in the same direction with the promoter of the responsive cassette placed close to one ITR (figure 10-0), and AAV UptkpSwS-UTEB Head to Head $(\mathrm{HtH})$ where the two promoters were arranged in the middle of the vector and the transcription goes in the two opposite directions from the centre to the ITRs of the vector (figure 10-n). In order to isolate a possible interaction between the two promoters 1 TB was inserted between them generating the AAV UptkpSwS-1TBUTEB HtH (figure 10-p). In the last construct the Uptk promoter from AAV UptkpSwS-UTEB HtH was substituted with the astrocytic promoter Gfabc1D generating AAV Gfabc1dpSwS-UTEB HtH (figure 10-q).

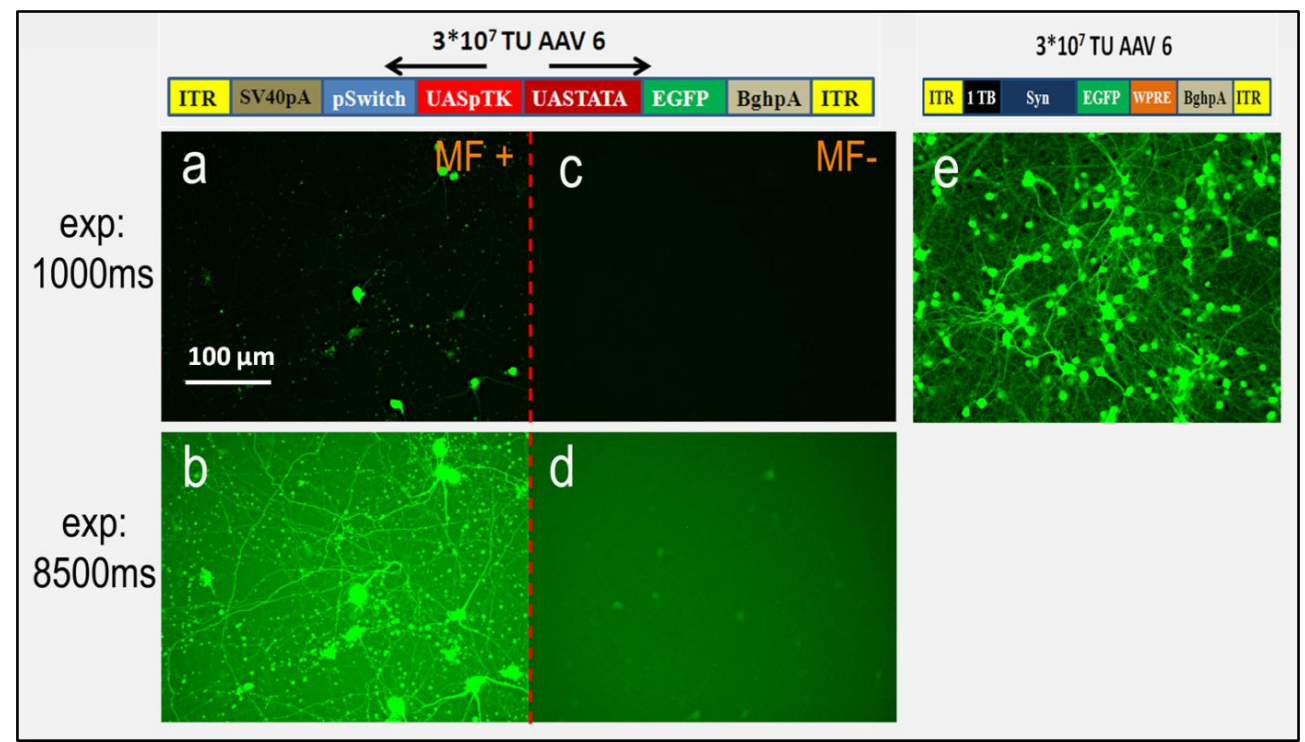

Figure 16) Cortical neurons infected with $3^{*} 10^{7} \mathrm{TU} /$ well of the pSwitch system packed in a unique AAV6 vector (pictures a-d) and AAV6 SEWB $3^{*} 10^{7} \mathrm{TU} /$ well (picture e). The induction of the system upon MF addition can be verified by comparing picture a versus $c$ (exp time $=1000 \mathrm{~ms}$ ) or picture b versus $d$ (exp time $=\mathbf{8 5 0 0} \mathrm{ms}$ ). The level of expression upon activation is much lower compared to neurons infected with AAV6 SEWB (picture e). Moreover an apoptotic like shape in picture $b$ can be seen.

As primary cortical neuron cultures contain only a few astrocytes, only the three viruses with the ubiquitous promoter were tested in vitro. For these experiments $3^{*} 10^{7} \mathrm{TU} /$ well were used and, as a positive control, AAV6 SEWB $\left(3 * 10^{7} \mathrm{TU} /\right.$ well) was added to the culture.

All three constructs gave similar results (figure 16), showing poor inducibility in the on-state (figure 16 pictures $a$ and $b$ versus e) and low but detectable EGFP expression in the off-state (figure 16 picture d). Moreover, induced neurons presented obvious neurite destruction, indicating a possible toxicity of this configuration of the system. 
Despite the uncertain results obtained in cell culture, the four viruses were tested in the rat striatum and, in order to avoid possible toxic effects, two titres were used: $2.75^{*} 10^{8} \mathrm{TU}$ (like in the previous experiments), and a lower titre of $1 * 10^{8} \mathrm{TU}$.

The first virus tested was AAV6 UptkpSwS-UTEB HtT (figure 10-o) and the following groups of animals were prepared:

a. AAV6 UptkpSwS-UTEB HtT $2.75 * 10^{8}$ not induced

b. AAV6 UptkpSwS-UTEB HtT $2.75 * 10^{8}$ induced with MF $20 \mathrm{mg} / \mathrm{kg}$

c. AAV6 UptkpSwS-UTEB HtT $1 * 10^{8}$ not induced

d. AAV6 UptkpSwS-UTEB HtT $1 * 10^{8}$ induced with MF $20 \mathrm{mg} / \mathrm{kg}$

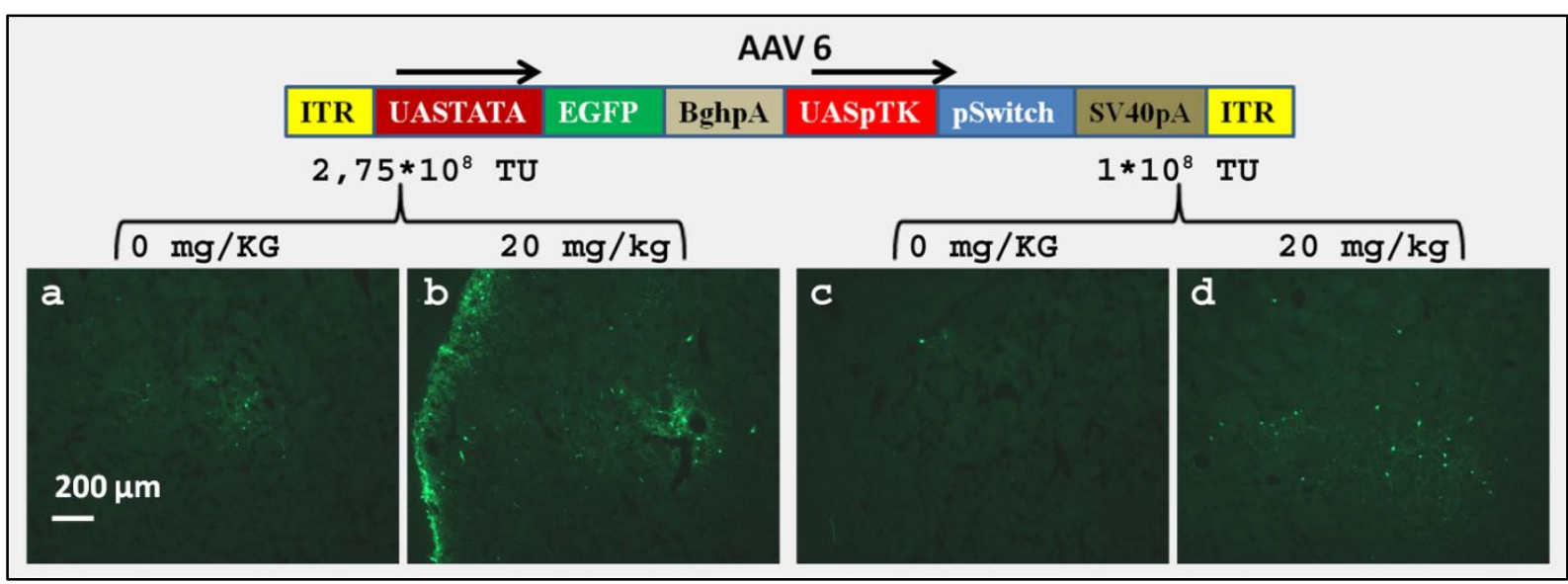

Figure 17) $25 \mu \mathrm{m}$ thick brain coronal sections of Wistar rats injected with $2.75 * 10^{8}$ (pictures a, b) and with $1 * 10^{8}$ (pictures c, d) of AAV6 UptkpSwS-UTEB HtT. The activation of the system upon MF induction can be assessed by comparing animals that did not received MF (pictures a and c) and animals that were induced with $20 \mathrm{mg} / \mathrm{kg}$ (pictures b and d). The EGFP expression, while low everywhere, depends on the titre of virus injected both without (picture a versus $c$ ) and with (picture $b$ versus d) MF induction. Background expression without induction is low but clearly detectable. Acquisition time $=\mathbf{5 0 0 0} \mathrm{ms}$.

Results of this experiment are reported in figure 17. The different EGFP expression level due to the amount of virus injected is shown comparing pictures $a$ and $b$ (high titre) versus pictures $c$ and $d$ (low titre). In both MF-induced groups the reporter gene expression is fairly low and in both not-induced groups EGFP expression without induction can be observed.

In conclusion AAV6 UptkpSwS-UTEB HtT is not satisfactory for both the induced and not-induced conditions. Problems might have arisen from an interaction between the ITR and the responsive promoter or an interaction between the two cassettes.

In order to avoid a possible promoter-ITR interaction, in the following experiment the vector AAV6 UptkpSwS-UTEB HtH (figure 10-n) with the two promoters placed in the middle of the vector was used. Four groups of animals were prepared:

a. AAV6 UptkpSwS-UTEB HtH $2.75 * 10^{8}$ not induced

b. AAV6 UptkpSwS-UTEB HtH $2.75 * 10^{8}$ induced with MF $20 \mathrm{mg} / \mathrm{kg}$

c. AAV6 UptkpSwS-UTEB HtH $1^{*} 10^{8}$ not induced

d. AAV6 UptkpSwS-UTEB HtH 1*10 ${ }^{8}$ induced with MF $20 \mathrm{mg} / \mathrm{kg}$ 
Results of this experiment are reported in figure 18. The different EGFP expression level due to the amount of virus injected is shown comparing pictures $a$ and $b$ (high titre) versus pictures $c$ and $d$ (low titre). The difference between the on and off-states is marked but the EGFP expression of the system, when not induced, is still detectable, although low.

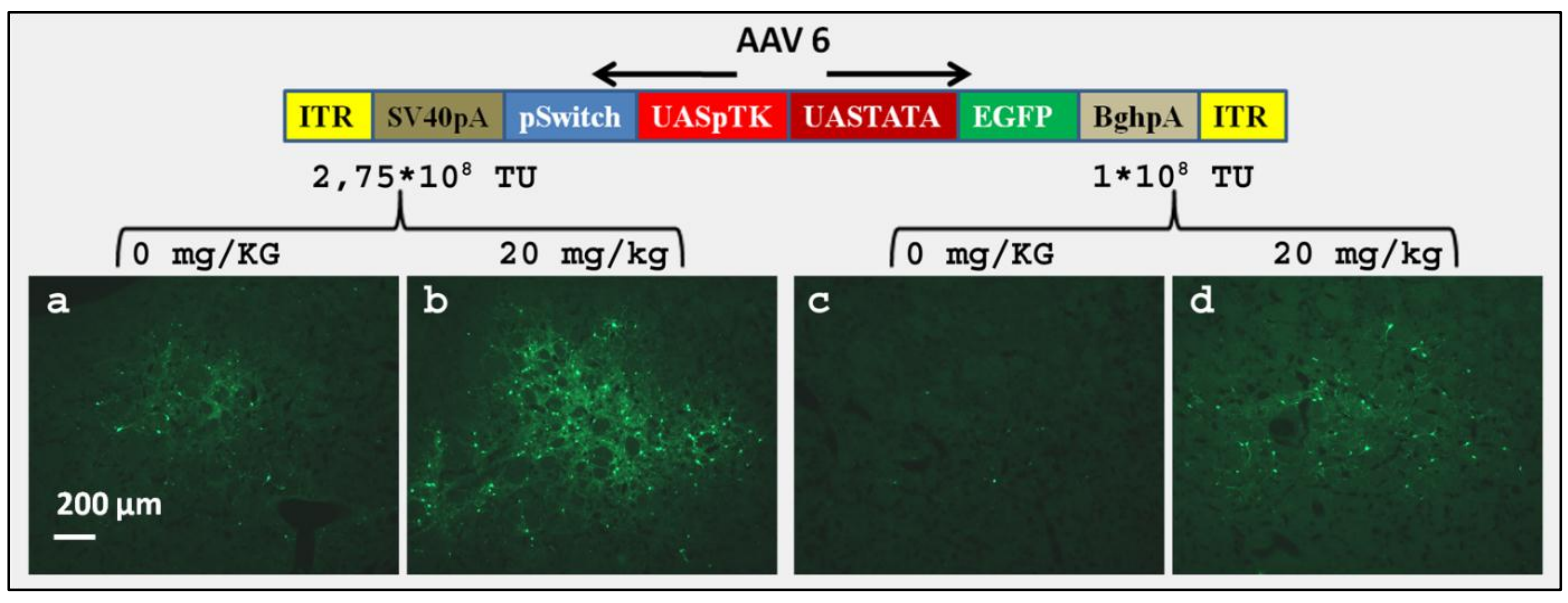

Figure 18) $25 \mu \mathrm{m}$ thick brain coronal sections of Wistar rats injected with $2.75 * 10^{8}$ (pictures a, b) and with $1 * 10^{8}$ (pictures c, d) of AAV6 UptkpSwS-UTEB HtH. The activation of the system upon MF induction can be assessed by comparing animals that did not received MF (pictures a and c) and animals that were induced with $20 \mathrm{mg} / \mathrm{kg}$ (pictures $b$ and d). The EGFP expression depends on the titre of virus injected both without (picture a versus $c$ ) and with (picture $b$ versus d) MF induction. Background expression without induction is high when the higher titre in used (picture a) and is still detectable when the lower titre is used (picture d). Acquisition time $=\mathbf{5 0 0 0} \mathbf{m s}$.

In conclusion, this virus, in vivo, shows a good induced level of EGFP expression but a still detectable EGFP expression in the off-state which might be due to the proximity of the two promoters (Uptk and UAS TATA) belonging to the two pSwitch cassettes; to investigate this possibility a transcription blocker was inserted between the two cassettes generating AAV6 UptkpSwS-1TB-UTEB (figure 10-p). In addition, in all the following experiments, some groups of animals will be induced and sacrificed one month later to evaluate the switching off of the system several weeks post induction. The following groups were prepared:

a. AAV6 UptkpSwS-1TBUTEB HtH $2.75 * 10^{8}$ not induced

b. AAV6 UptkpSwS-1TBUTEB HtH $2.75 * 10^{8}$ induced with MF $20 \mathrm{mg} / \mathrm{kg}$

c. AAV6 UptkpSwS-1TBUTEB HtH $2.75 * 10^{8}$ induced with MF $20 \mathrm{mg} / \mathrm{kg}+1$ month

d. AAV6 UptkpSwS-1TBUTEB HtH $1 * 10^{8}$ not induced

e. AAV6 UptkpSwS-1TBUTEB HtH $1 * 10^{8}$ induced with MF $20 \mathrm{mg} / \mathrm{kg}$

f. AAV6 UptkpSwS-1TBUTEB HtH $1 * 10^{8}$ induced with MF $20 \mathrm{mg} / \mathrm{kg}+1$ month 
As with the previous experiments, the EGFP expression is correlated with the amount of virus injected; this can be seen by comparing the EGFP level in animals induced with MF (figure 19 picture $b$ versus e). In not induced animals a slight leakiness is observed only in animals injected with the higher titre of virus (figure 19 picture a); animals injected with the lower titre display an almost undetectable EGFP expression (figure 19 picture d). Similar background fluorescence can be observed in animals that were sacrificed one month after induction (figure 19 pictures $\mathrm{c}$ and $\mathrm{f}$ ).

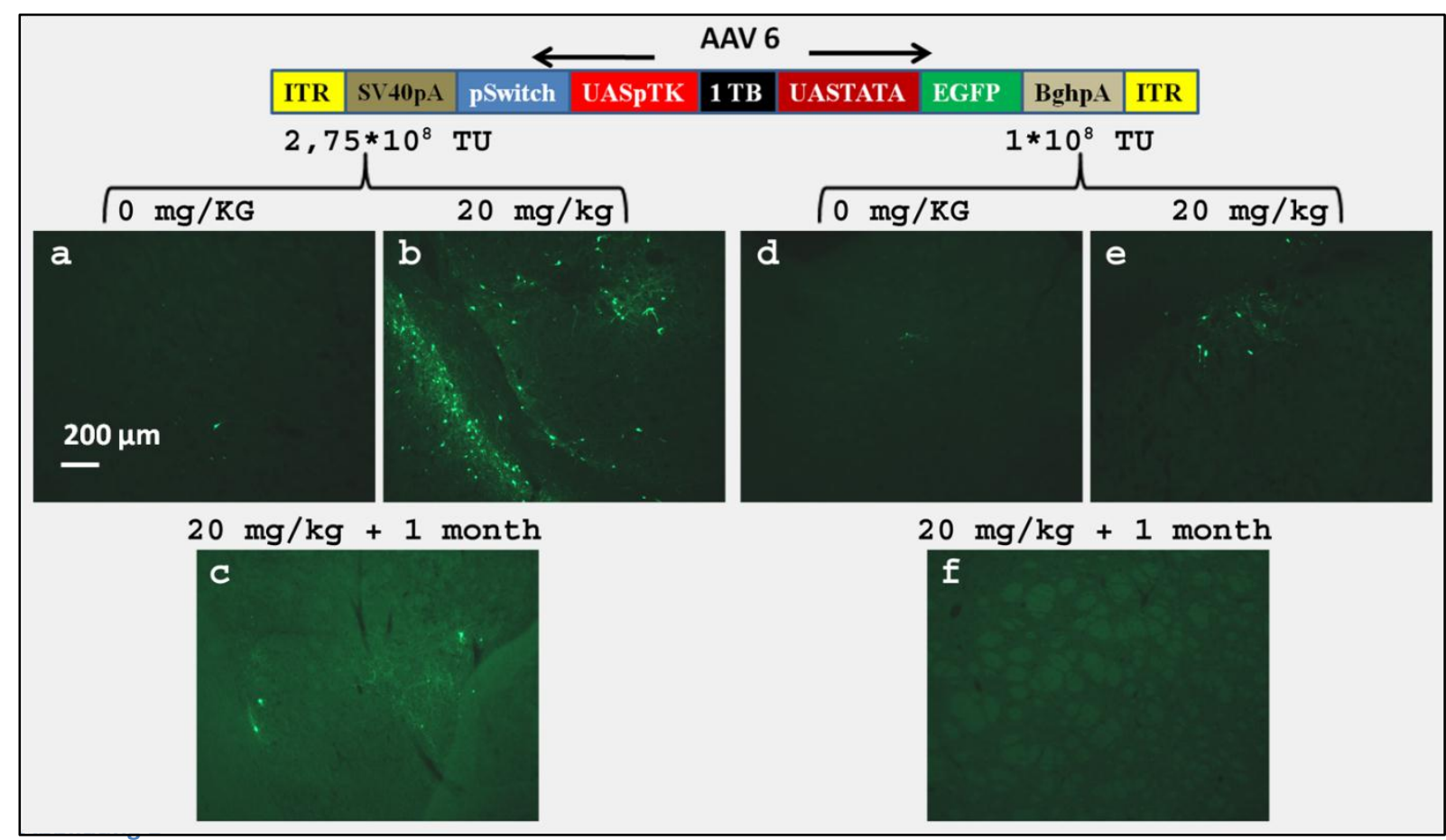

Figure 19) $25 \mu \mathrm{m}$ thick brain coronal sections of Wistar rats injected with $2.75 * 10^{8}$ (pictures a-c) and with $1 * 10^{8}$ (pictures d-f) of AAV6 UptkpSwS-1TBUTEB HtH. The activation of the system upon MF induction can be assessed by comparing animals that did not received MF (pictures a and d) and animals that were induced with $20 \mathrm{mg} / \mathrm{kg}$ (pictures $b$ and e). The EGFP expression level depends on the amount of virus injected (picture a versus $d$ and $b$ versus e). As can be seen in pictures $\mathrm{c}$ and $\mathrm{f}$ the expression of the reporter protein returns to basal level in animals sacrificed 1 month after induction. The basal level of EGFP expression in animals injected with $1 * 10^{8}$ of the virus is almost undetectable while it is present in animals injected with $2.75 * 10^{8}$ TU. Acquisition time $=5000 \mathrm{~ms}$.

In conclusion these experiments showed that a good compromise between on- and off-states with this virus is not achievable. By injecting a small amount of the virus we observe very low background of EGFP expression in the off-state but, upon MF-induction only few EGFP expressing neurons are observed; in order to obtain a good level of EGFP expression in the on-state one must inject more virus, which leads to a detectable EGFP expression in the not-induced state.

In order to restrict the expression only to astrocytes the ubiquitous promoter Uptk was substituted with the astrocytic specific promoter Gfabc1d. The obtained vector was named AAV6-Gfabc1dpSwSUTEB (figure 10-q). The following four groups of animals were prepared:

a. AAV6 Gfabc1dpSwS-UTEB HtH $2.75 * 10^{8}$ not induced

b. AAV6 Gfabc1dpSwS-UTEB HtH $2.75^{*} 10^{8}$ induced with MF $20 \mathrm{mg} / \mathrm{kg}$

c. AAV6 Gfabc1dpSwS-UTEB HtH $1 * 10^{8}$ not induced

d. AAV6 Gfabc1dpSwS-UTEB HtH $1 * 10^{8}$ induced with MF $20 \mathrm{mg} / \mathrm{kg}$ 
In accordance with previous results the level of EGFP expression depends on the amount of virus injected; this is shown in figure 20 both by comparing the non-induced animals (figure 20 picture a versus $c$ ) and the induced ones (figure 20 picture $b$ versus $d$ ). The virus shows, with both titres, a good level of EGFP expression when the animal is induced and a moderate background when animals are not-induced.

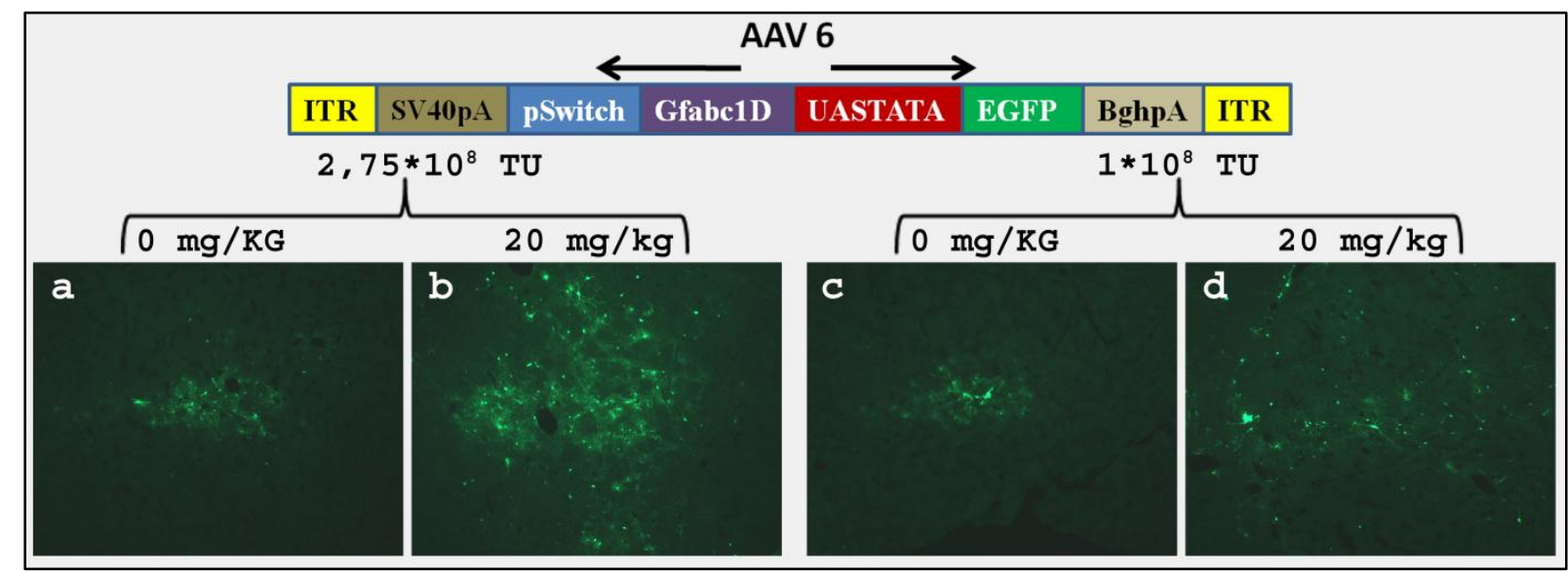

Figure 20) $25 \mu \mathrm{m}$ thick brain coronal sections of Wistar rats injected with $2.75 * 10^{8}$ (pictures a and b) and with $1 * 10^{8}$ (picture $c$ and d) of AAV6 Gfabc1dpSwS -UTEB HtH. The activation of the system upon MF induction can be assessed by comparing animals that did not received MF (pictures a and c) and animals that were induced with $20 \mathrm{mg} / \mathrm{kg}$ (pictures b and d). The EGFP expression depends on the titre of virus injected both without (picture a versus $c$ ) and with (picture $b$ versus d) MF induction. Background expression without induction remains high. Acquisition time $=\mathbf{5 0 0 0} \mathbf{m s}$.

As all the viruses tested show a variable leakage in the not-induced state, the next step consisted on packing the vectors in AAV5 capsid instead of AAV6. As previously mentioned AAV5 serotype is known to spread more and deliver less genome per cell comparing to AAV6 [111] which should lead to a decrease of expression in both states.

\subsubsection{One vector system in AAV5}

As previously explained AAV5 viruses do not transducer efficiently in primary cortical neuron culture and, as a consequence, vectors were tested only in vivo.

Initially, due to the good compromise between the on and the off-states observed with the vectors packed in AAV6 viruses, only the two Head to Head vectors without TB were investigated in AAV5 (AAV UptkpSwS-UTEB HtH (figure 10-n) and AAV5 Gfabc1dpSwS-UTEB HtH (figure 10-q)).

Animals injected with AAV5 UptkpSwS-UTEB HtH were divided in the following groups:

a. AAV5 UptkpSwS-UTEB HtH $2.75 * 10^{8}$ not induced

b. AAV5 UptkpSwS-UTEB HtH $2.75 * 10^{8}$ induced with MF $20 \mathrm{mg} / \mathrm{kg}$

c. AAV5 UptkpSwS-UTEB HtH $2.75 * 10^{8}$ not induced +1 month

d. AAV5 UptkpSwS-UTEB HtH $2.75 * 10^{8}$ induced with MF $20 \mathrm{mg} / \mathrm{kg}+1$ month

e. AAV5 UptkpSwS-UTEB HtH $1 * 10^{8}$ not induced

f. AAV5 UptkpSwS-UTEB HtH $1 * 10^{8}$ induced with MF $20 \mathrm{mg} / \mathrm{kg}$

g. AAV5 UptkpSwS-UTEB HtH $1 * 10^{8}$ not induced +1 month

h. AAV5 UptkpSwS-UTEB HtH $1 * 10^{8}$ induced with MF $20 \mathrm{mg} / \mathrm{kg}+1$ month

Figure 21 shows that animals that received higher amount of virus (figure 21 picture b) show higher EGFP expression upon MF induction compared to animals injected with lower amounts of virus 
(figure 21 picture f). EGFP expression in the off-state is undetectable in both cases (figure 21 pictures a and e). All groups of animals that were sacrificed 1 month after induction (figure 21 pictures $c, d, g$, h) show an undetectable level of EGFP expression demonstrating the ability of the pSwitch system to silence the transgene expression once the inducer is metabolized.

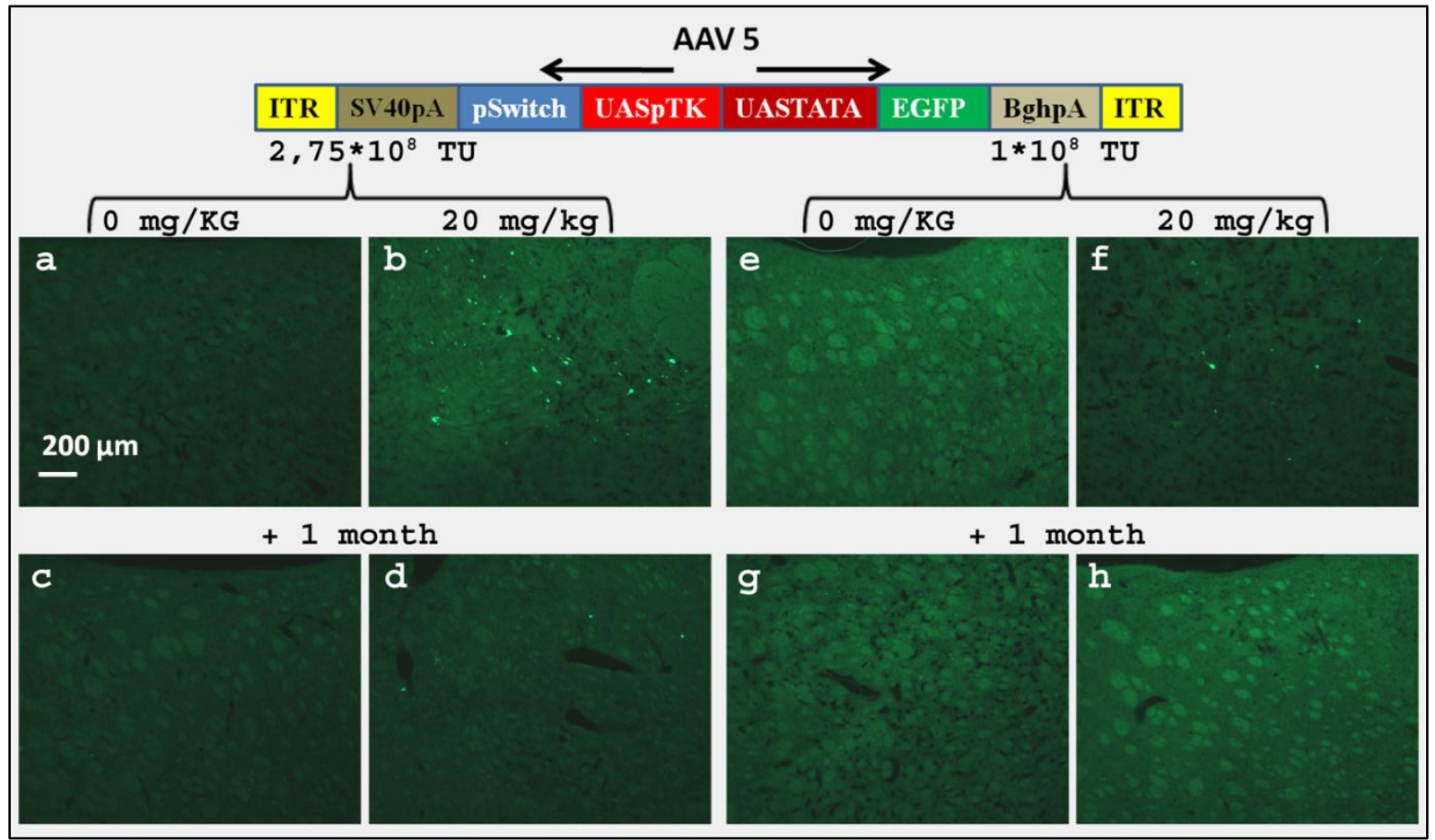

Figure 21) $25 \mu \mathrm{m}$ thick brain coronal sections of Wistar rats injected with $2.75 * 10^{8}$ (picture a-d) and with $1 * 10^{8}$ (pictures e-h) of AAV5 UptkpSwS -UTEB HtH. The activation of the system upon MF induction can be assessed by comparing animals that did not received MF (pictures a and e) and animals that were induced with $20 \mathrm{mg} / \mathrm{kg}$ (pictures $b$ and f). The EGFP expression depends on the titre of virus injected and can be verified comparing picture $b$ versus $f$. The basal level of expression is undetectable while it is moderate upon MF induction. In all cases the EGFP expression goes to basal level in animals sacrificed one month after induction (pictures $c, d, g, h$ ). Acquisition time $=10000 \mathrm{~ms}$.

In conclusion AAV5 UptkpSwS-UTEB HtH displays a moderate level of expression when induced, an almost undetectable level of expression when non-induced, and a return to an undetectable level of EGFP expression 1 month after the drug induction.

As mentioned above, in order to restrict the expression of the pSwitch system only to astrocytes the vector Gfabc1dpSwS-UTEB HtH (figure 10-q) was generated and the following groups of animals were prepared:

a. AAV5 Gfabc1dpSWS-UTEB HtH $2.75 * 10^{8}$ not induced

b. AAV5 Gfabc1dpSwS-UTEB HtH $2.75 * 10^{8}$ induced with MF $20 \mathrm{mg} / \mathrm{kg}$

c. AAV5 Gfabc1dpSwS-UTEB HtH $2.75 * 10^{8}$ not induced +1 month

d. AAV5 Gfabc1dpSwS-UTEB HtH $2.75 * 10^{8}$ induced with MF $20 \mathrm{mg} / \mathrm{kg}+1$ month

e. AAV5 Gfabc1dpSWS-UTEB HtH $1 * 10^{8}$ not induced

f. AAV5 Gfabc1dpSwS-UTEB HtH $1 * 10^{8}$ induced with MF $20 \mathrm{mg} / \mathrm{kg}$

g. AAV5 Gfabc1dpSwS-UTEB HtH $1 * 10^{8}$ not induced +1 month

h. AAV5 Gfabc1dpSwS-UTEB HtH $1 * 10^{8}$ induced with MF $20 \mathrm{mg} / \mathrm{kg}+1$ month 
As can be deduced from figure 22 the EGFP expression of this virus differs completely from the previously tested viruses. Indeed the EGFP expression in induced animals appears stronger in animals injected with a lower titre of virus compared to rats that received the higher dosage of the vector (figure 22 picture $b$ versus $\mathrm{f}$ ). Moreover, animals that received a high titre of virus, and that were sacrificed one month after induction, showed a strong EGFP expression independently of whether they had received the MF induction or not (figure 22 pictures $c$ and d); this phenomenon was not seen in animals injected with low titre (figure 22 pictures $g$ and $h$ ). Due to this unexpected performance the virus was not further investigated.

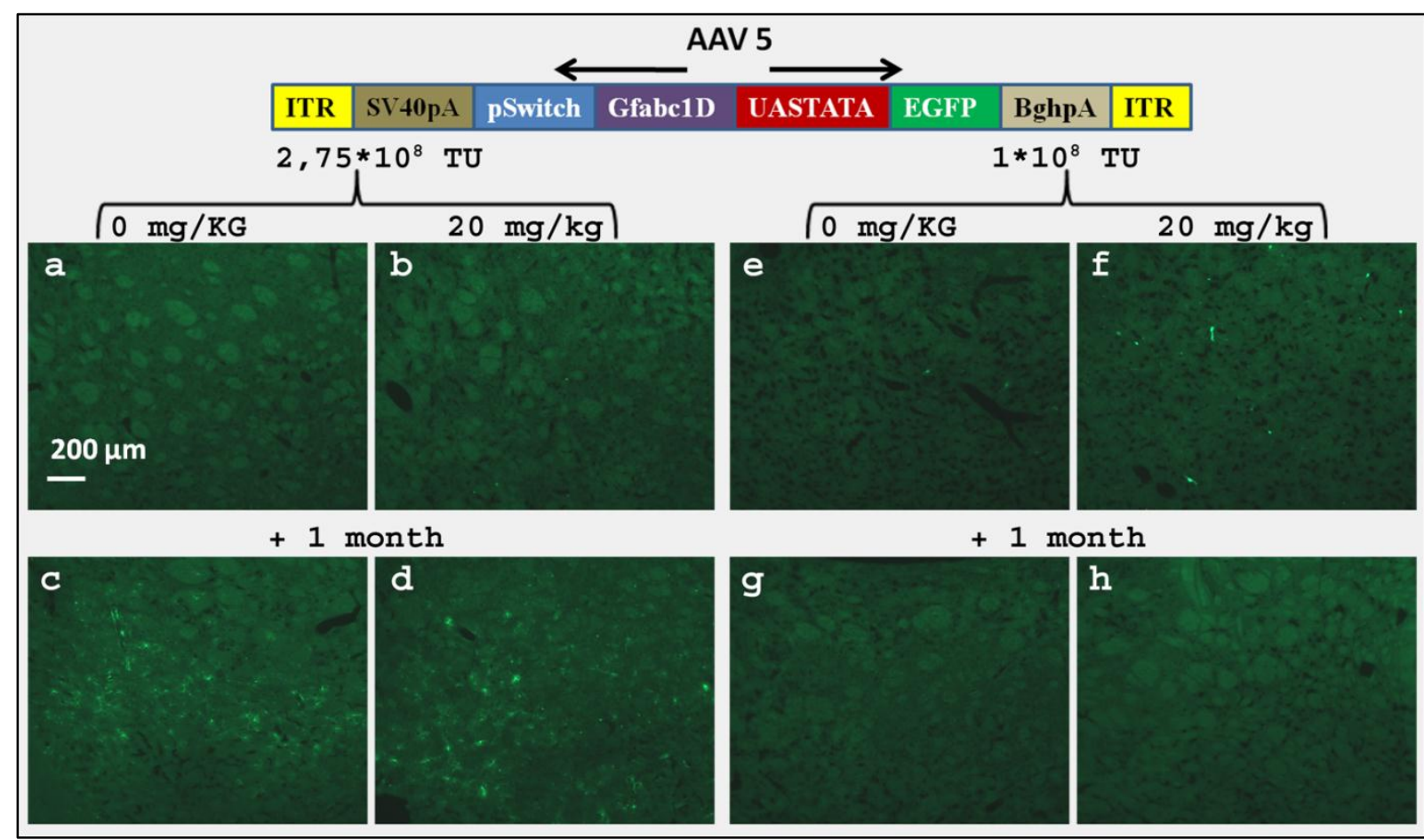

Figure 22) $25 \mu \mathrm{m}$ thick brain coronal sections of Wistar rats injected with $2.75^{*} 10^{8}$ (pictures a-d) and with $1 * 10^{8}$ (pictures e-h) of AAV5 Gfabc1dpSwS -UTEB HtH. The EGFP expression is detectable only in animals injected with $1 * 10^{8}$ and induced with MF (picture f) and in animals injected with $2,75 * 10^{8}$ of the virus and sacrificed one month after induction (pictures $c$ and d). EGFP expression is undetectable in all other groups indicating an uncontrollable behavior of the system. Acquisition time $=\mathbf{1 0 0 0 0} \mathrm{ms}$.

\subsubsection{Two vectors system in AAV5}

The one vector system packed in AAV5 showed the possibility to achieve a very low background expression but we could not obtain a substantial EGFP expression upon induction. As a good level of transgene expression was obtained with the two vector configuration using AAV6 serotype, in the following experiment we tested the performance of the system using a two vector system packed in AAV5 serotype virus. As a consequence of the previous experiments performed with AAV6 vectors only the responsive vector with 3TB and without WPRE was used (AAV 3TB UTEB figure 10-I). Three different promoters were used to express the pSwitch protein in order to evaluate differences in the EGFP expression and the possibility to restrict the expression only to defined populations of cells: The ubiquitous promoter Uptk (figure 10-f), the astrocytic promoter Gfabc1d (figure 10-g), and the neuron specific promoter synapsin (figure 10-h). 
In the first experiment the inducing vector with the ubiquitous promoter Uptk (AAV5 1TB UptkpSwS figure 10-f) in combination with the responsive vector AAV5 3TB UTEB (figure 10-I) was used and the following groups of animals were prepared:

a. AAV5 -3TB-UTEB $1 * 10^{8}+$ AAV5 UptkpSwS $0.3^{*} 10^{8}$ not induced

b. AAV5 -3TB-UTEB $1 * 10^{8}+$ AAV5 UptkpSwS $0.3 * 10^{8}$ induced with MF $20 \mathrm{mg} / \mathrm{kg}$

c. AAV5 -3TB-UTEB $1 * 10^{8}+$ AAV5 UptkpSwS $0.3 * 10^{8}$ not induced +1 month

d. AAV5 -3TB-UTEB $1 * 10^{8}+$ AAV5 UptkpSwS $0.3 * 10^{8}$ induced with MF $20 \mathrm{mg} / \mathrm{kg}+1$ month

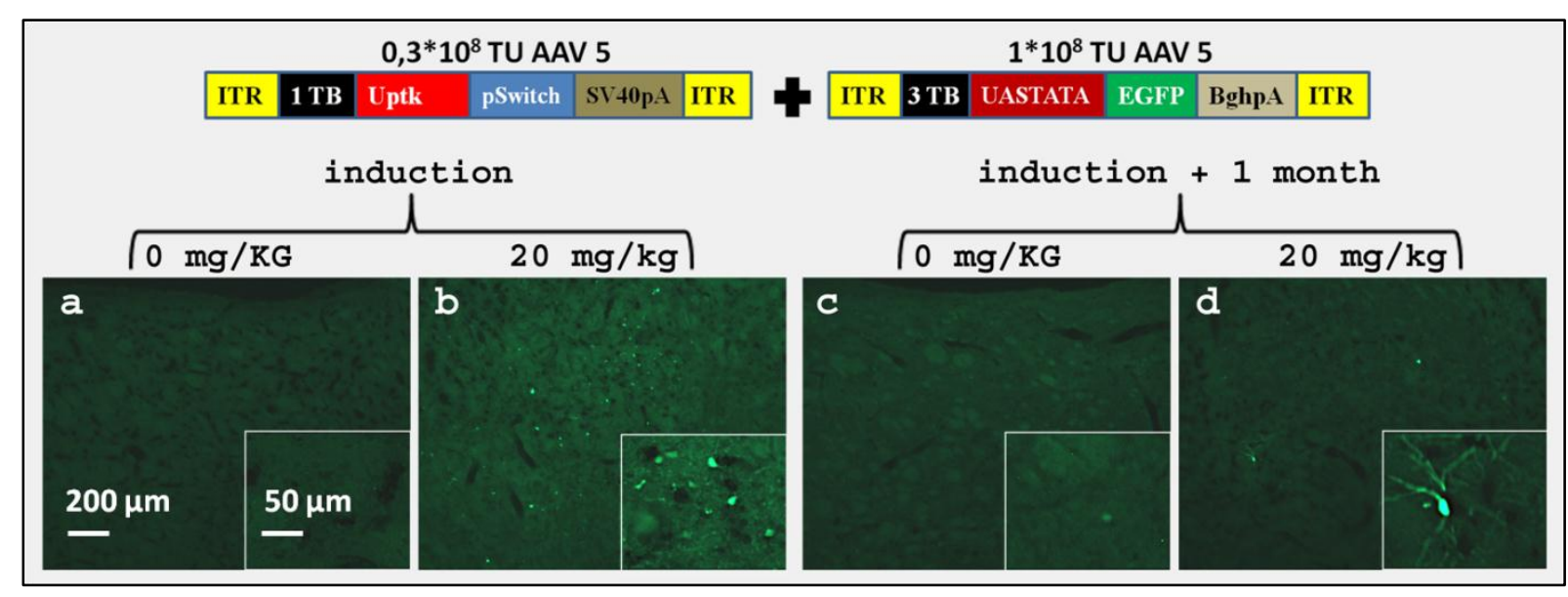

Figure 23) $25 \mu \mathrm{m}$ thick brain coronal sections of Wistar rats injected with AAV5 -3TB-UTEB $1 * 10^{8}+$ AAV5 UptkpSwS $0.3^{*} 10^{8}$. The activation of the system upon MF induction can be assessed by comparing animals that did not received MF (picture a) and animals that were induced with $20 \mathrm{mg} / \mathrm{kg}$ (picture b). Animals sacrificed one month after induction (pictures $c$ and d) show a decrease of the EGFP expression to background level except for some cells which still express EGFP. Background expression without induction is almost undetectable (picture a). Acquisition time $=10000 \mathrm{~ms}$.

In figure 23 we can observe: 1) the EGFP expression in non-induced animals is almost undetectable (picture a); 2) a clear activation of the system upon MF injection (picture a versus b); 3 ) animals sacrificed one month after induction show a decrease of EGFP expression to basal level except for some cells (pictures $\mathrm{c}$ and $\mathrm{d}$ ) which still express the reporter protein.

In conclusion, this combination of viruses shows an acceptable compromise between the on- and the off-states but the EGFP expression after one month appears to be not completely silenced.

In order to restrict the expression of the pSwitch protein only to astrocytes the Uptk promoter in the inducing plasmid was substituted with the Gfabc1d promoter (figure 10-g) and the obtained vector was injected in the rat striatum together with the AAV 3TB-UTEB (figure 10-I) responsive vector; animals were divided in the following groups:

a. AAV5 -3TB-UTEB $1 * 10^{8}+$ AAV5 Gfabc1dpSwS $0.3 * 10^{8}$ not induced

b. AAV5 -3TB-UTEB $1 * 10^{8}+$ AAV5 Gfabc1dpSwS $0.3 * 10^{8}$ induced with MF $20 \mathrm{mg} / \mathrm{kg}$

c. AAV5 -3TB-UTEB $1 * 10^{8}+$ AAV5 Gfabc1dpSwS $0.3 * 10^{8}$ not induced +1 month

d. AAV5 -3TB-UTEB $1 * 10^{8}+$ AAV5 Gfabc1dpSwS $0.3 * 10^{8}$ induced with MF $20 \mathrm{mg} / \mathrm{kg}+1$ month 


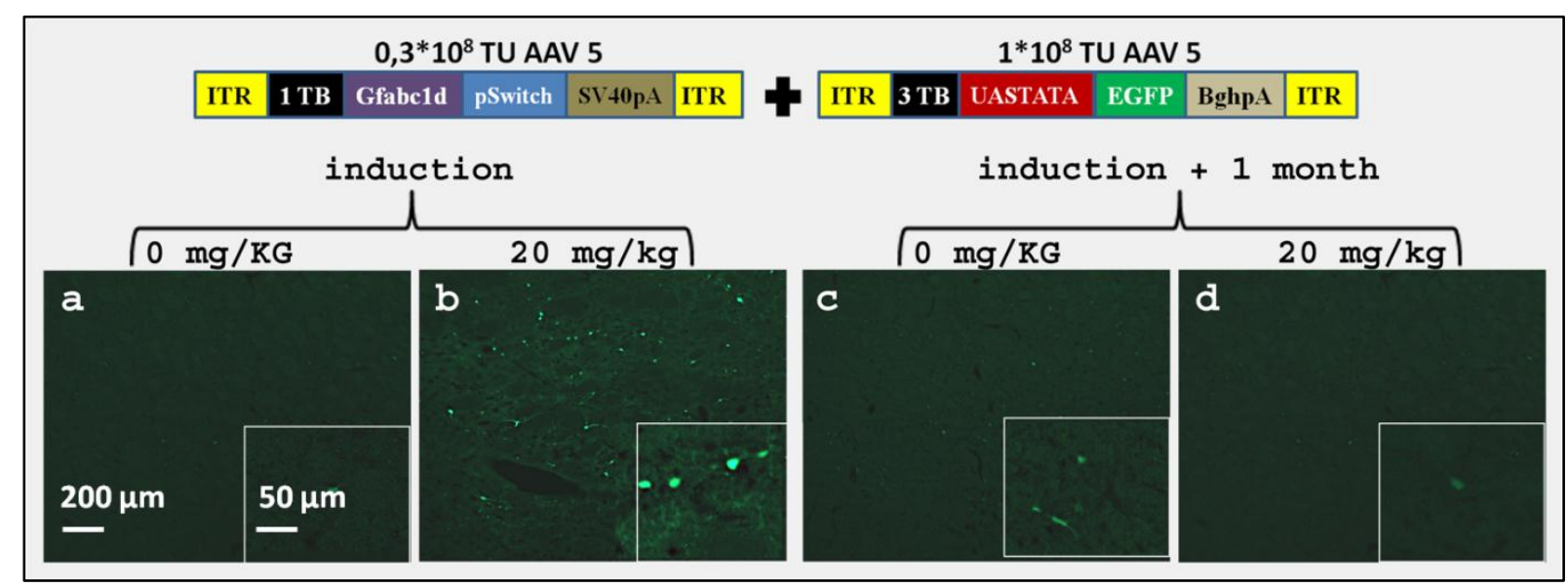

Figure 24) $25 \mu \mathrm{m}$ thick brain coronal sections of Wistar rats injected with AAV5 -3TB-UTEB $1 * 10^{8}+$ AAV5 Gfabc1dpSwS $0.3^{*} 10^{8}$. The activation of the system upon MF induction can be assessed by comparing animals that did not received MF (picture a) and animals that were induced with $20 \mathrm{mg} / \mathrm{kg}$ (picture b). Animals sacrificed one month after induction (pictures $c$ and d) show a decrease of the EGFP expression to background level in which the presence of the reporter protein is almost undetectable. Acquisition time $=10000 \mathrm{~ms}$.

Figure 24 shows that the EGFP expression in induced animals is clearly visible (figure 24 picture a versus $b$ ) while the expression in non induced animals (figure 24 picture a) or in animals that were induced and then sacrificed one month later (figure 24 pictures $\mathrm{c}$ and d) is almost undetectable.

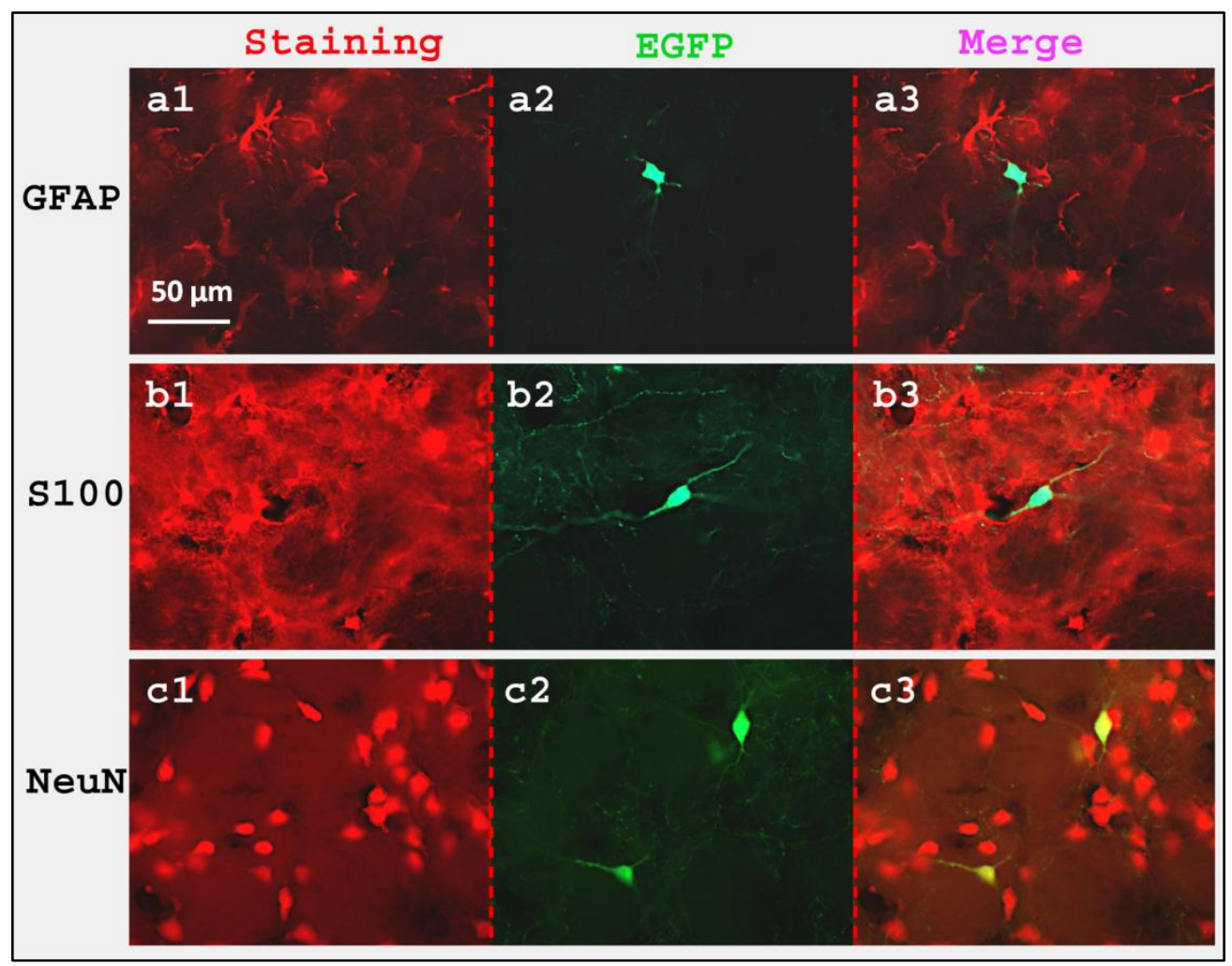

Figure 25) Immunostaining on EGFP positive cells in the pSwitch system. EGFP expressing cells seems to be neurons: GFAP staining (panel a) and S100 staining (panel b) do not give double positive cells while the NeuN staining (panel c) in overlapping with EGFP. a1, b1, c1 = staining; a2, b2, c2 = EGFP; a3, b3, c3 = merge.

In order to assess the specificity of the Gfabc1d promoter three different immunostaining were performed; GFAP and S100 to identify astrocytes and NeuN to highlight neurons. As is demonstrated in figure 25 the different immunostainings suggest that EGFP positive cells are neurons. Indeed only 
the NeuN staining (figure 25 panel c) is co-localizing with EGFP expressing cells while the GFAP (figure 25 panel a) and the S100 (figure 25 panel b) are not. Thus, in the contest of AAV vectors the promoter is far less astrocyte-specific as compared to transgenic mice [101].

Despite the lack of specificity this combination of viruses gave the desired compromise between the on and the off state.

In order to restrict the EGFP expression only to neurons, the astrocytic promoter Gfabc1d was replaced with the neuronal specific synapsin promoter and the vector AAV SpSwS (figure 10-h) was obtained. This vector was used together with the responsive virus AAV5 3TB-UTEB (figure 10-I) and two groups of animals were prepared:

a) AAV5 -3TB-UTEB $1 * 10^{8}+$ AAV5 SynpSwS $0.3 * 10^{8}$ not induced

b) AAV5 -3TB-UTEB $1 * 10^{8}+$ AAV5 SynpSwS $0.3^{*} 10^{8}$ induced with MF $20 \mathrm{mg} / \mathrm{kg}$

As shown in figure 26, the expression of EGFP is almost undetectable in not induced animals (figure 26 picture a) while is clearly visible in induced animals (figure 26 picture b).

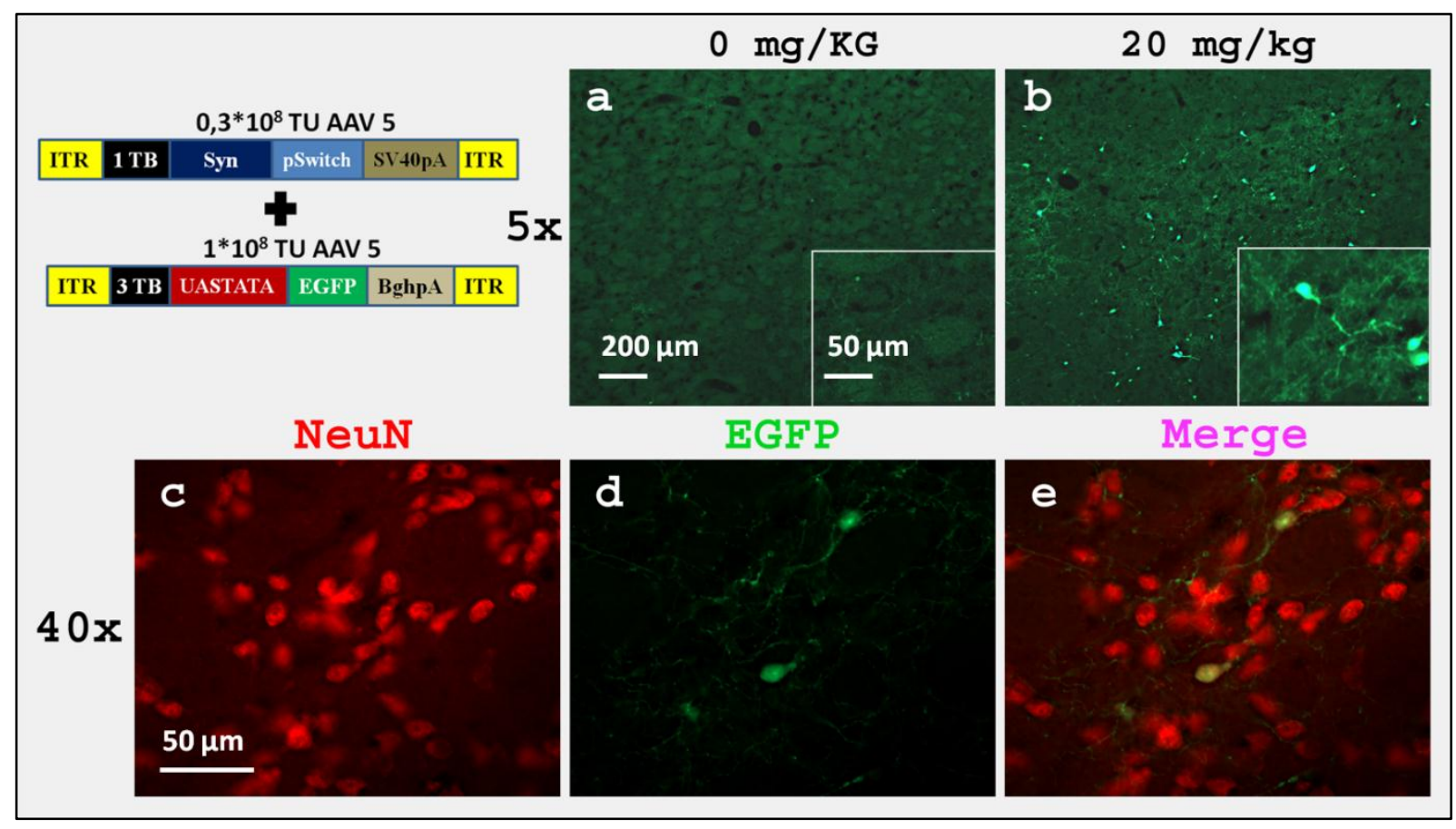

Figure 26) Transduction in rat striatum with AAV5 -3TB-UTEB $1 * 10^{8}+$ AAV5 SynpSwS $0.3 * 10^{8}$. The inducibility of the system is confirmed by picture a $(0 \mathrm{mg} / \mathrm{Kg} \mathrm{MF})$ versus picture $b(20 \mathrm{mg} / \mathrm{Kg} \mathrm{MF})$. The specificity of the promoter is verified by the NeuN staining (c) which is overlapping with the EGFP expression (d) in the merge picture (e). Acquisition time a and $b=10000$ ms; Acquisition time c-e = "automatic".

The neuronal specific expression was assessed by IHC using as antigen the neuronal specific marker NeuN; the co-localization of EGFP and NeuN is demonstrated in figure 26 picture e.

This combination of viruses, for neuron specific expression of the transgene, resulted in a good compromise between the on- and the off-state and the EGFP expression appears to be cells specific. 


\subsubsection{Conclusions}

Two different serotypes and two different concepts in assembling the expression cassettes for the pSwitch protein and regulated transgene have been tested. When AAV6 serotype was used a good level of EGFP expression was achieved but it was not possible to avoid leakage of the system with either the one or the two vector system. The use of the one vector system in the AAV5 vectors displayed a very low background expression but a good level of expression upon activation was not possible. An acceptable compromise between the on- and the off-state was obtained by packing the two pSwitch cassettes in two separate AAV5 vectors; in the following experiment for studying MF dosage and reiterate induction of the pSwitch system AAV5 3TB-UTEB (figure 10-I) was used as a responsive vector and AAV5 Gfabc1dpSwS (figure 10-g) as inducing vector. The neuronal specific inducing vector AAV5 SynpSwS (figure 10-h) was used in the experiments involving GDNF expression.

\subsection{Study on mifepristone (MF) dosage}

According to manufacturer's specifications, the pSwitch system displays the following properties when used in cell culture: a) the truncated progesterone receptor has an affinity for the ligand mifepristone of $1 * 10^{-9} \mathrm{M}, \mathrm{b}$ ) the system should be fully activated at a steroid concentration of $10 \mathrm{nM}$, and c) the system should be $50 \%$ induced when the concentration of MF is $0.1 \mathrm{nM}$.

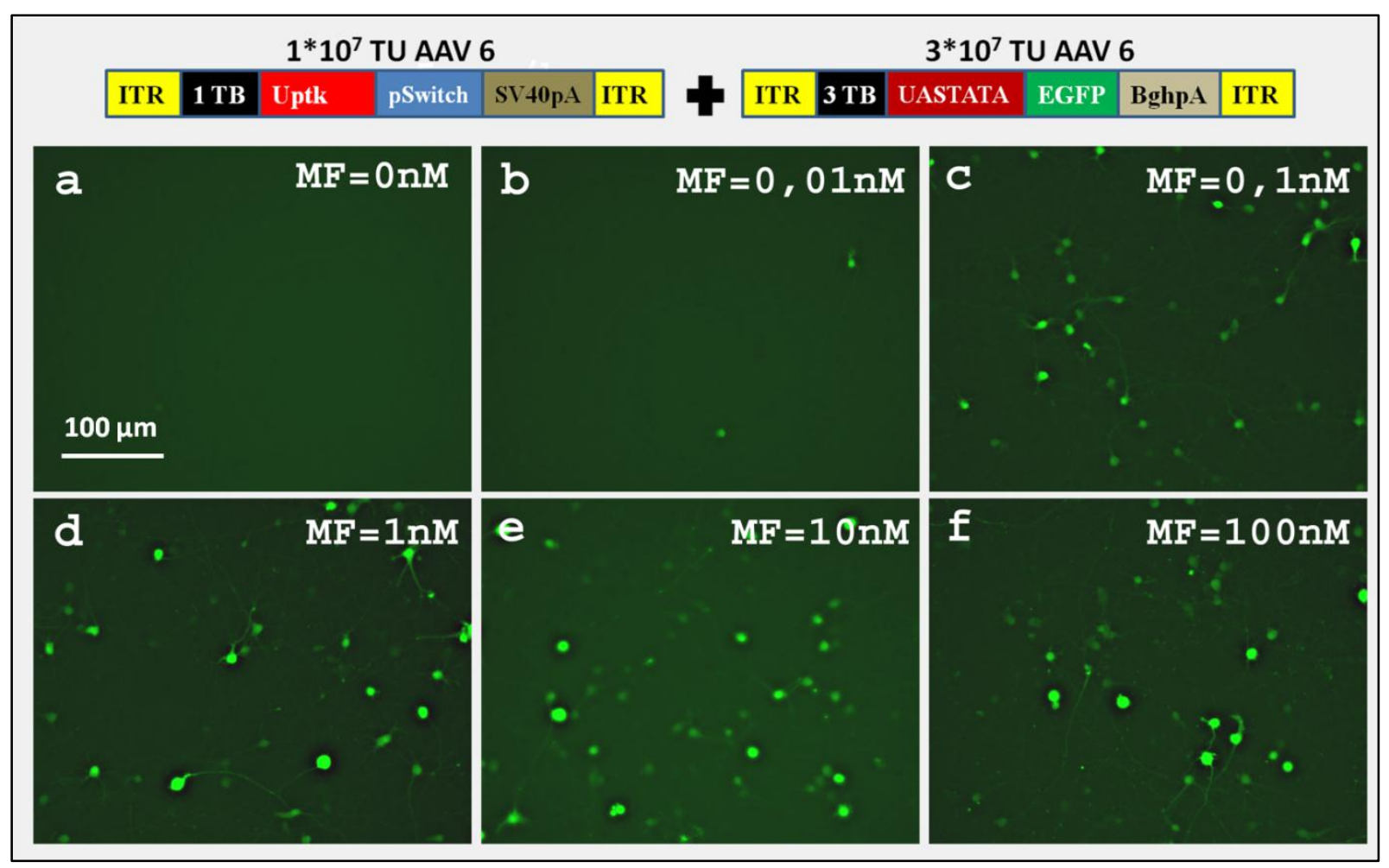

Figure 27) Mifepristone dosage in primary cortical neurons. Neurons were transduced with $1 * 10^{7}$ AAV6 UptkpSwS + 3*10 7 AAV6 3TB UTEB on DIV3, MF was added on DIV7 and pictures were acquired on DIV12. MF concentration ranged from 0 to $100 \mathrm{nM}$ in a 10-log scale. The threshold appears to be between 0.01 and $0.1 \mathrm{nM}$ (pictures $b$ and $c$ ) while higher dosage do not show evident differences (pictures $d$, $e$ and $f$ )

Primary cortical neurons were infected with $1 * 10^{7}$ AAV6 UptkpSwS $+3 * 10^{7}$ AAV6 3TBUTEB and the MF was added at a concentration between $0.01 \mathrm{nM}$ and $100 \mathrm{nM}$ on a 10-log scale. Figure 27 demonstrates: a) the system displays some activation already at a steroid concentration of $0.01 \mathrm{nM}$ (figure 27 picture b); b) the activation window is clearly between 0.01 (figure 27 picture b) and 0.1 
$\mathrm{nM}$ (figure 27 picture c); c) no obvious difference in EGFP expression was detected in cells induced with a MF concentration between 0.1 and $100 \mathrm{nM}$ (figure 27 pictures $c, d, e, f$ ).

In order to evaluate the MF dose effect when the pSwitch system is used in the rat striatum, four groups of animals were injected with $3 * 10^{7}$ AAV5 GfABC1DpSwS (figure 10-g) $+1 * 10^{8}$ AAV5 3TB UTEB (figure 10-I). In previous experiments we had already assessed that the use of a MF dosage of $50 \mathrm{mg} / \mathrm{kg}$ displays some side effects; thus, in this experiment, animals were induced with 20, 10, 5 and $0 \mathrm{mg} / \mathrm{kg}$ of the drug. In figure 28 pictures a-d it is shown that the system is activated with all the dosages tested and that the EGFP expression seems dose dependent. To quantify the fluorescence we noted that, in a $5 x$ picture, $95 \%$ of the pixels are background and only $5 \%$ of them represent the actual EGFP expression. By averaging the intensity of those pixels we can estimate the amount of EGFP expressed. In the graph in picture e the obtained averaged intensity was subtracted with the intensity calculated in not induced animals. The increase in fluorescence is highly significant $(p<$ 0.005 with 2 tailed t-Test) between the dosages of 0-5 and 5-10 while the difference between the 10 and the $20 \mathrm{mg} / \mathrm{kg}$ dosage is not significant $(p=0.87$ ) if analyzed with a 2 tailed t-Test. This difference became significant $(p<0.05)$ if the data are analyzed with a one tail t-Test indicating a tendency of increased expression in the $20 \mathrm{mg} / \mathrm{kg}$ dosage group.

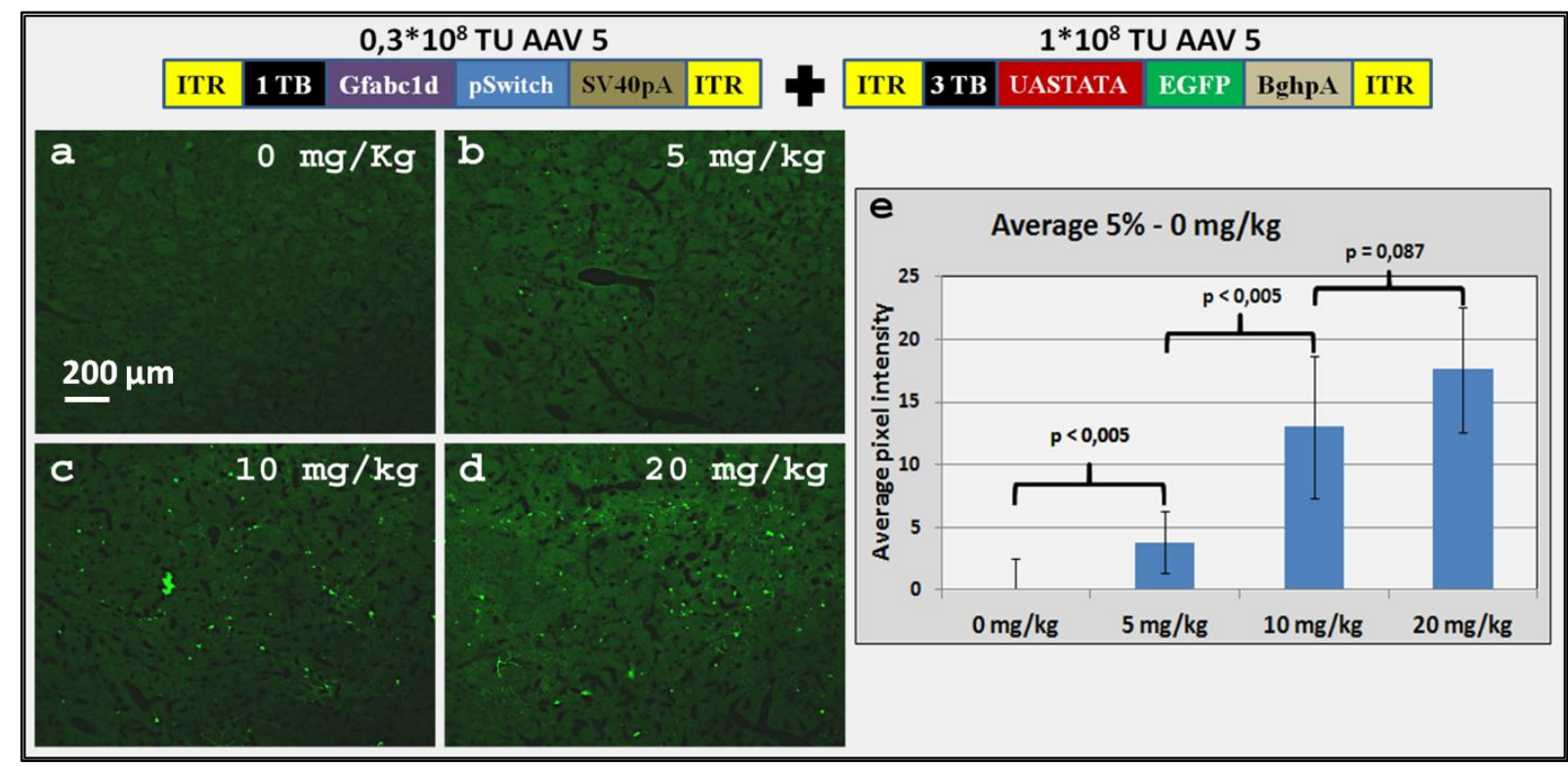

Figure 28) Study of MF dosage in rat striatum; pictures a-d $=25 \mu \mathrm{m}$ coronal sections. Acquisition time $=10000 \mathrm{~ms}$. Animals were injected with $1 * 10^{7}$ AAV5 GfABC1DpSwS $+1 * 10^{8}$ AAV5 3TB UTEB and were induced with 0 (picture a), 5 (picture b), 10 (picture c) and 20 (picture d) $\mathrm{mg} / \mathrm{kg}$ of MF. A dependency of the dosage can be observed. Graph in picture e shows the average pixel intensities when only the $5 \%$ brightest pixels are quantified. In this graph the value obtained with the $0 \mathrm{mg} / \mathrm{kg}$ dosage was considered to be zero. The two tailed t-test was applied. Error bars = standard deviation.

\subsection{Toxicity of the pSwitch system in cell culture}

To test for toxicity of the viruses and of the inducer MF, cortical neurons were plated in 96 wells/plates and infected with the viruses. Non infected neurons were used as a control and neurons infected with AAV6 SEWB were used as a second control to assess the toxicity due to transduction; results are reported in the graph in figure 29.

In the samples that were not treated with MF, a $20 \%$ decrease in viability compared to untreated neurons was measured in most of the samples transduced with viruses. As this decrease is present in the second control (neurons infected with AAV6 SEWB) as well it can be assumed that it is due to the 
transduction procedure by itself. In two cases the viability decreases substantially in comparison with the second control (neurons infected with AAV6 SEWB): 1) neurons infected with AAV6 Uptk-pSw-SUTEB HtH showed a $25 \%$ viability in comparison to the control confirming the impression of toxicity observed in microscopy analysis (chapter 4.2.2 "One vector system in AAV6") and 2) neurons transduced only with high titre of AAV6 Uptk-pSw-S showed a 40\% decrease comparing to non infected neurons; the high titre was used in order to match the titre of the one vector system $\left(3^{*} 10^{7}\right.$ TU/well).This difference might explain the lack of toxicity of the vector when used at a lower amount in combination with the different responsive vectors in the two vector system.

The addition of the inducer MF decreases the viability in all samples (except for the AAV6 Uptk-pSwS-UTEB HtH probably because the viability is already really low) indicating a toxic effect of MF or EtOH (MF for cell culture is diluted in EtOH) in cell culture.

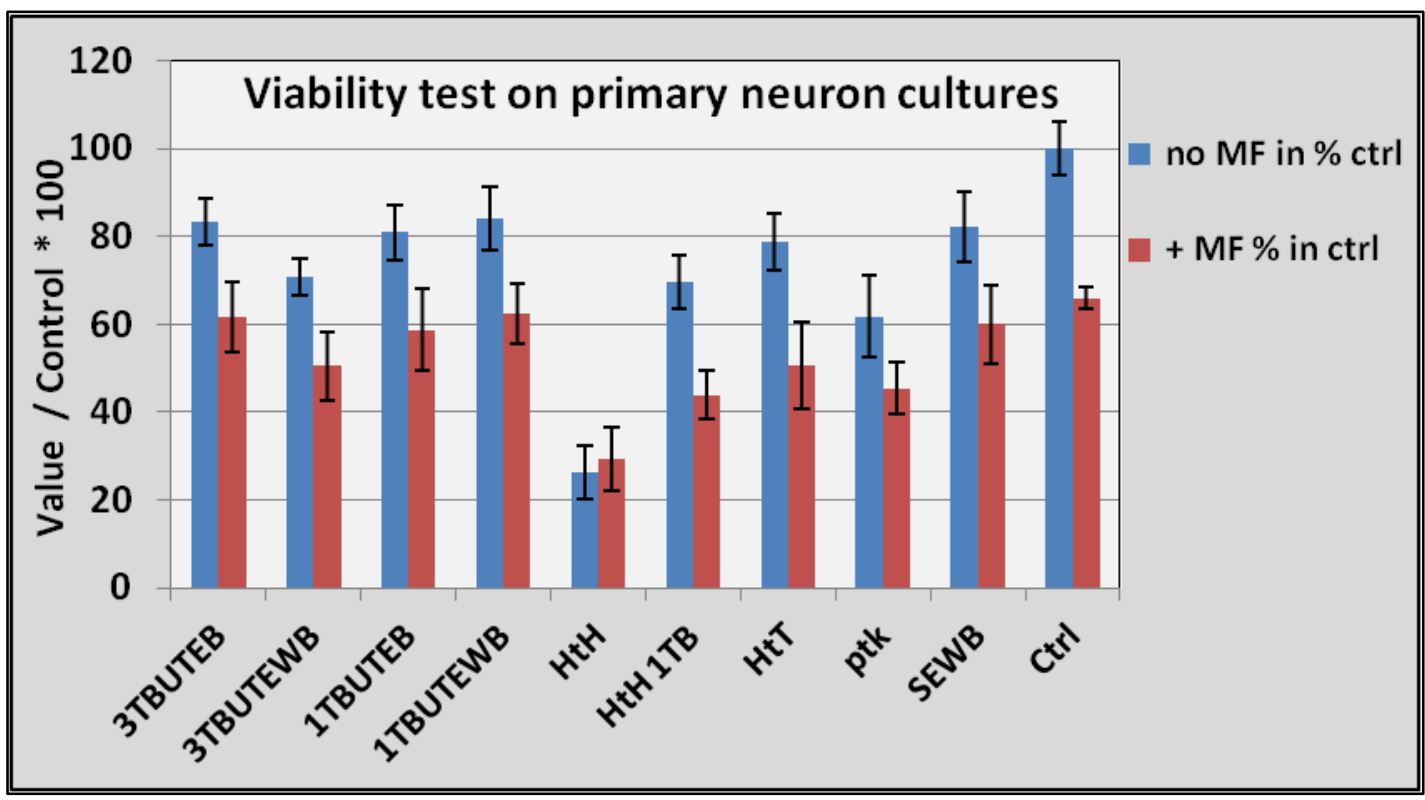

Figure 29) Viability test on cortical neurons in culture transduced with different combination of the pSwitch system. Viability was normalized with control non-transduced neurons. Neurons viability is strongly impaired when the one vector system $\mathrm{HtH}$ or when only the inducing vector Uptk used at high titre are used. 3TBUTEB = AAV6 UptkpSwS + AAV6 3TB UTEB (1:3); 3TBUTEWB = AAV6 UptkpSWS + AAV6 3TB UTEWB (1:3); 1TBUTEB = AAV6 UptkpSwS + AAV6 1TB UTEB (1:3); 1TBUTEWB = AAV6 UptkpSwS + AAV6 1TB UTEwB (1:3); HtH = AAV6 UptkpSwS-UTEB head to head; HtH 1TB = AAV6 UptkpSwS-1TBUTEB head to head; HtT = AAV6 UptkpSwS-UTEB head to tail; ptk = AAV6 UptkpSwS (high titre); SEWB = AAV6 SEWB (EGFP constitutively expressing virus); Ctrl = control non-treated neurons; $\mathrm{MF}=$ mifepristone. Error bars $=$ standard deviation.

\subsection{Reiterate activation of the pSwitch system in vivo}

The previous results have shown that the system can be activated upon MF addition and that EGFP fluorescence decreases to basal level 1 month after induction. However, the aim of the project was to develop a system that can undergo several cycles of activation/deactivation over the time, characteristic essential for clinical application.

Here, using various strategies, we show the ability of the pSwitch system in AAV to fulfill this requirement. 


\subsubsection{Multiple induction in rat striatum}

To evaluate the reiterate induction of the pSwitch system in the rat striatum, animals were injected with $1 * 10^{8}$ AAV5 3TB-UTEB (figure 10-I) $+0.3 * 10^{8}$ AAV5 Gfabc1dpSwS (figure 10-g). Animals were sacrificed at different time points corresponding to when the system was supposed to be on and off (figure 30).

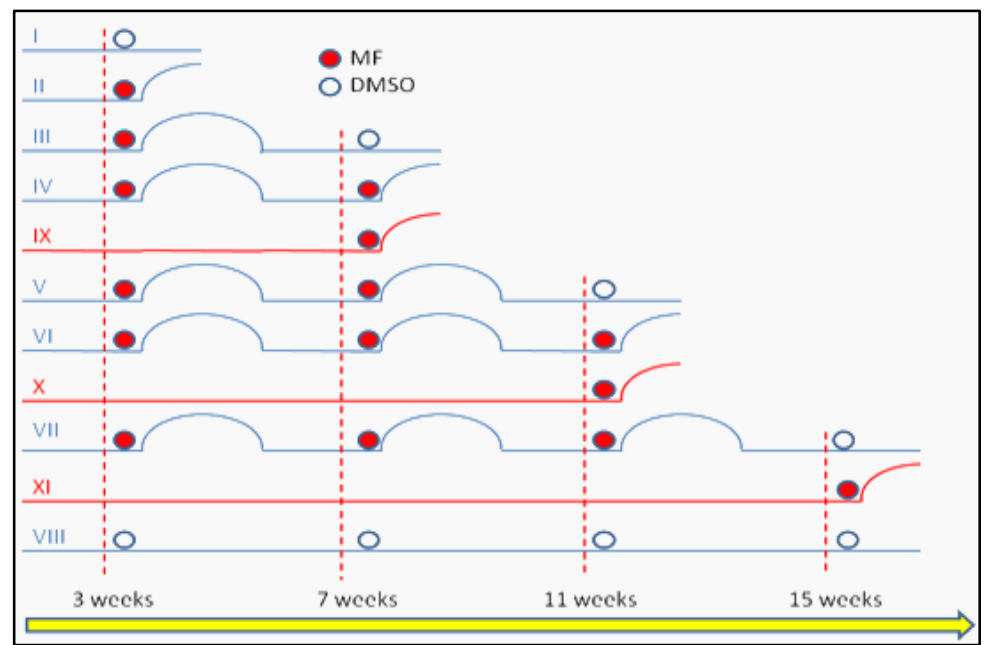

Figure 30) Time table of multiple induction experiment. Different group of animals were induced with MF (red dots) or with DMSO (white dots) and sacrificed at different time points. The ends of the blue lines represent the time point when animal were sacrificed; if the line is curved the system was supposed to be active while with the flat line we expect not to have EGFP expression. Red lines refer to control groups. MF = mifepristone.

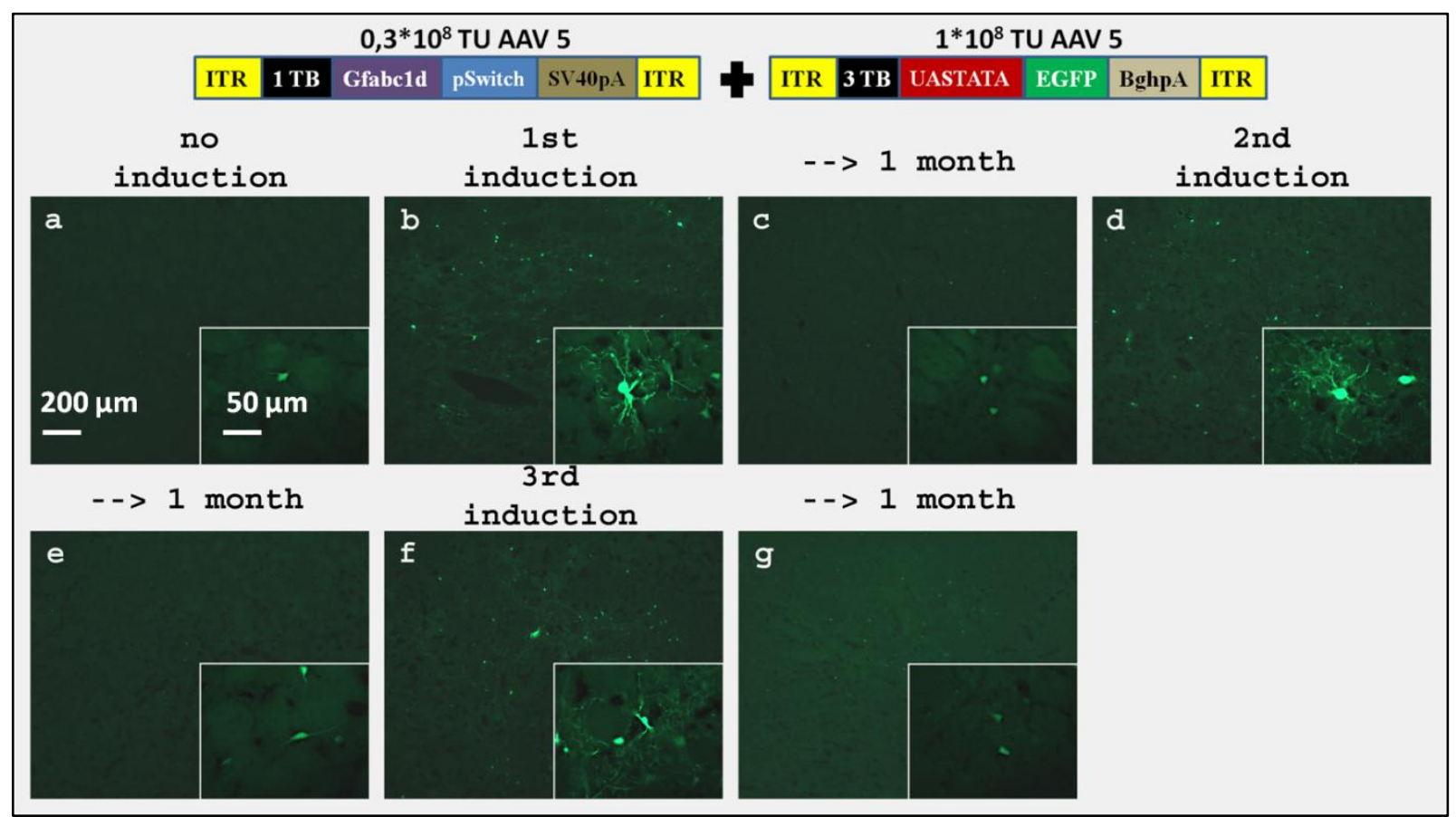

Figure 31) Reiterate induction of the pSwitch system. $25 \mu \mathrm{m}$ thick brain coronal section of Wistar rats injected with $1 * 10^{8}$ AAV5 3TB-UTEB $+0.3 * 10^{8}$ AAV5 Gfabc1dpSwS. Referring to the time table in figure 30 pictures $\mathrm{b}$, $\mathrm{d}$, and $\mathrm{f}$ represent the three inductions, pictures $c$, e and $g$ represent animals sacrificed 1 month after the last injection and picture a represents animals that did not receive any induction. The capacity of the system to undergo to several cycles of induction is evident. Acquisition time $=10000 \mathrm{~ms}$. 
As shown in figure 31 the system responds positively to all three cycles of induction (figure 31 pictures $b, d, f$ ) and the EGFP expression returns to not-induced level (figure 31 picture a) 4 weeks after induction (figure 31 pictures $\mathrm{c}, \mathrm{e}, \mathrm{g}$ ). No difference in EGFP expression between experimental and control groups was detected no loss of induction efficiency of the system over the consecutive cycles (data not shown).

\subsubsection{Reiterate imaging in mouse cortex with 2-photon microscopy}

With the aim to study the course of the EGFP expression in the same animal, viruses were injected into the mouse cortex, a cranial window was mounted over the injection point and pictures were acquired using a 2-photon microscope. As the cranial window is easier to apply to mice than rats, this technique was performed only on mice. These experiments were performed in parallel with the screening of different vectors and most of the viruses tested in rat striatum were tested in mice as well. Here only a representative selection of the results obtained using AAV6 vectors is presented; AAV6, due to the higher EGFP expression, was preferred to AAV5 for these experiments.

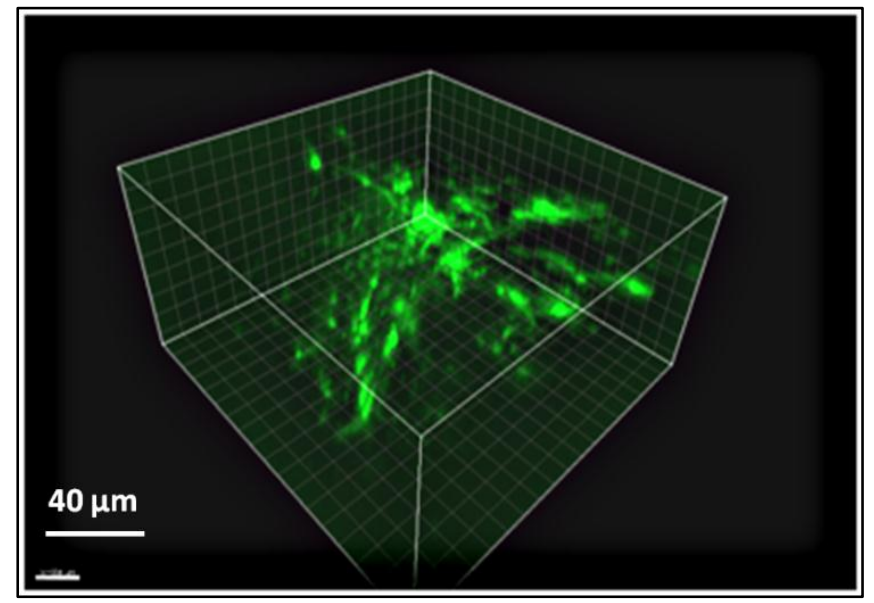

Figure 32) 3D reconstruction of a Z-Stack acquired with a 2-photon microscope on an animal injected with AAV6 GfABC1D EWB. The stack starts on the surface of the cortex and goes to a depth of $\sim 300 \mu \mathrm{m}$

As a positive control animals were injected with $1 * 10^{8}$ AAV6 Gfabc1dEWB. A 3D reconstruction of a Z-stack acquired with the 2-photon microscope is shown in figure 32; the acquisition started on the surface of the cortex and went to a depth of about $300 \mu \mathrm{m}$. Cell bodies and protrusions can be identified.

In a subsequent experiment mice were injected with $5 * 10^{7}$ AAV6 3TBUTEWB $+1 * 10^{7}$ AAV6 UptkpSwS; animals were imaged a first time, injected with $20 \mathrm{mg} / \mathrm{kg}$ of MF, imaged a second time and then imaged again after 2 weeks. As shown in figure 33 the same area can be easily found over the different imaging sessions. The EGFP expression is clearly increases after the MF induction and decreases in the last imaging session.

The fluorescence in the off-state was expected as the imaged animal was injected with AAV6 viruses and the responsive vector contained the WPRE element. 


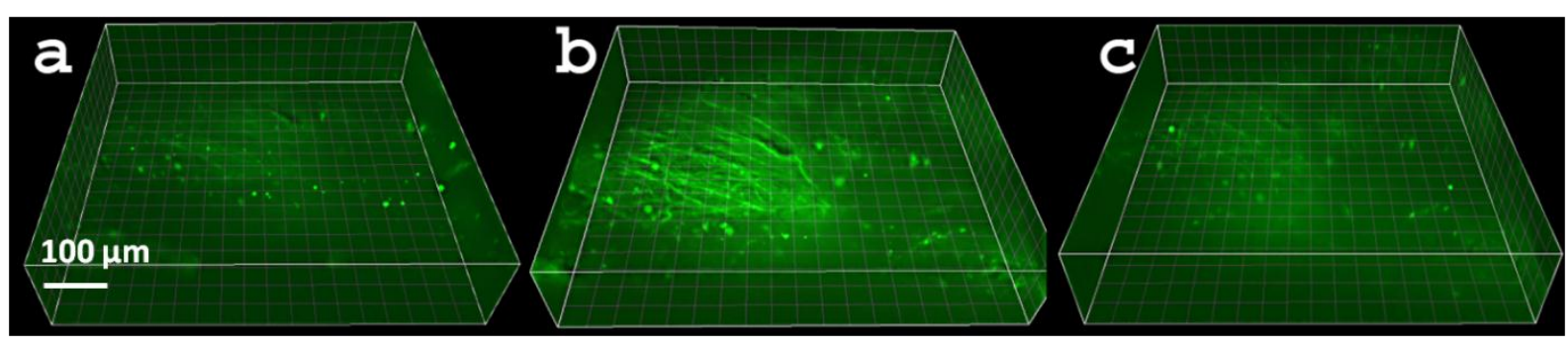

Figure 33) 3D reconstructions of the same cortex area of an animal injected with $5^{*} 10^{7}$ AAV6 3TBUTEWB $+1 * 10^{7}$ AAV6 UptkpSwS. Z-Stacks were taken before MF induction (picture a), 3 days after MF induction (picture b) and 3 weeks after MF induction (picture c). The same area can be easily identified and the responsiveness of the system to MF is evident.

\subsubsection{Reiterate imaging in mice cortex with the $0 \mathrm{~V}-100$ microscope}

Due to technical problems and availability of the 2-photon microscope, we decided to couple the cranial window technique together with the use of an OV100 microscope. The use of this microscope simplified the reiterate imaging at the expense of resolution.

As already mentioned AAV5 spreads more compared to AAV6; this characteristic, although advantageous for our purpose in previous experiments, was problematic for in vivo imaging. When injected in the cortex, EGFP expressing AAV5 viruses spread considerably and EGFP fluorescence was observed in the whole cranial window area. Due to this sparse expression it was difficult to identify the points of injection and compare the EGFP expression with the background fluorescence of the tissue. For this reason we only present here results obtained using AAV6 viruses which, spreading less, presented EGFP expression only in the proximity of the injection points rendering comparison between the transduced area and the background easier.

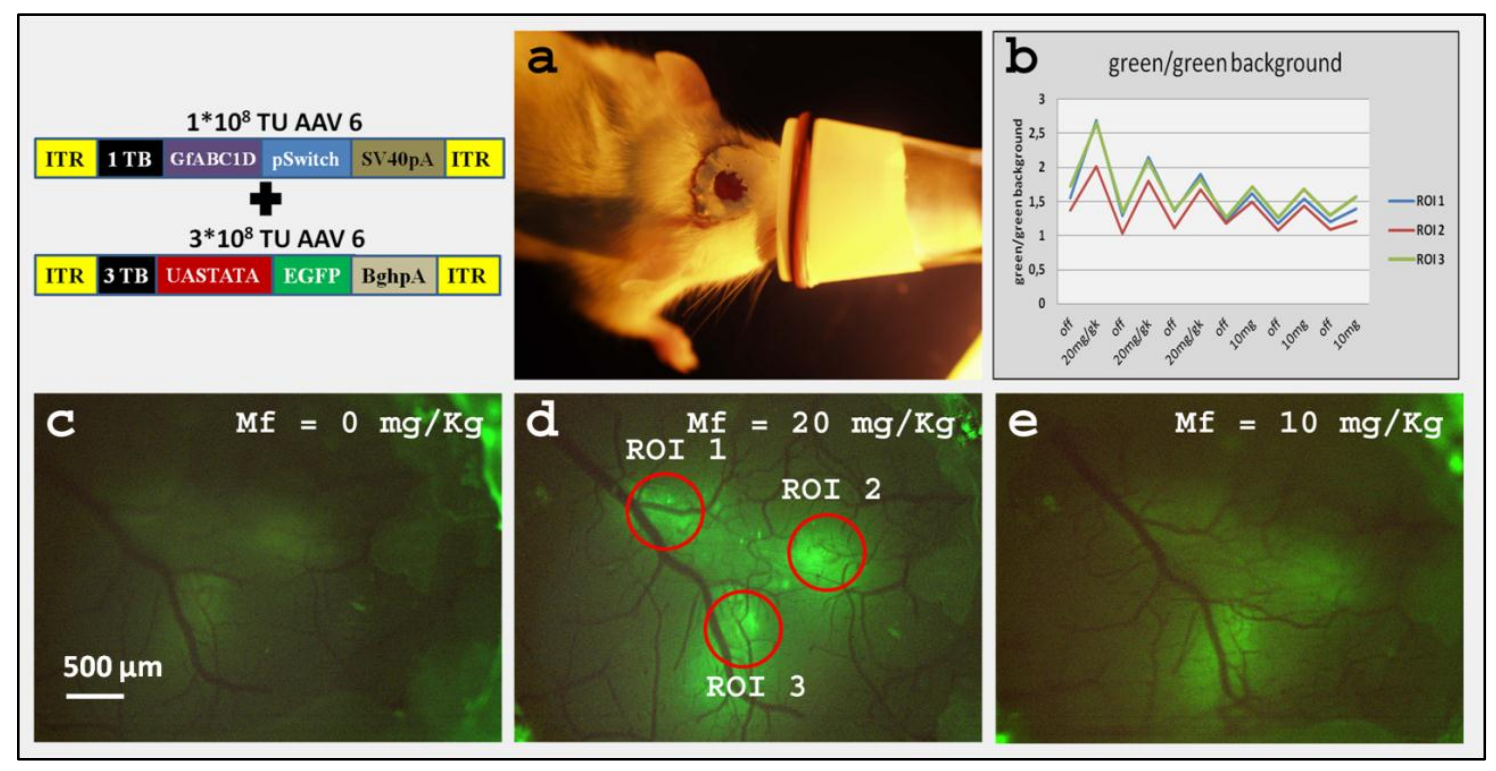

Figure 34) EGFP imaging with an OV-100 microscope. The mouse was previously injected in three points with $1 * 10^{8}$ TU AAV6 Gfabc1dpSwS $+3 * 10^{8}$ TU AAV\& 3TBUTEB. The animal was then anesthetized using isofluoran and placed in the imaging chamber (picture a). The difference in fluorescence in the three points of injection ( ROI 1, 2 and 3) can be clearly seen when the animal is injected with DMSO (picture c), with $\mathbf{2 0}$ (picture d) or with 10 (picture e). The fluorescence was measured, normalized versus the background and plotted in a graph (picture b). $\mathrm{MF}=$ mifepristone; $\mathrm{ROI}=$ region of interest 
Animals were injected with the viruses and imaged two weeks later to define the proper exposure time and camera parameters for each animal (figure 34 picture a). Animals were then induced every three weeks with 10 or $20 \mathrm{mg} / \mathrm{kg}$ of $\mathrm{MF}$ and were imaged before induction and 3 days after induction. Images were then processed using ImageJ [108] and the average fluorescence in the area of injection was normalized versus the background and plotted in a graph (figure 34 picture b).

In figure 34 the results obtained from an animal injected in three different points with $1 * 10^{8} \mathrm{TU}$ AAV6 Gfabc1dpSwS (figure 10-g) $+3 * 10^{8}$ TU AAV6 3TBUTEB (figure 10-I) are shown. The background fluorescence before induction is expected due to the use of AAV6 viruses (figure 34 picture $c$ ); the mouse was induced three times with $20 \mathrm{mg} / \mathrm{kg}$ of MF (figure 34 picture d) and three times with 10 $\mathrm{mg} / \mathrm{kg}$ of the drug (figure 34 picture e). The fluorescence in the three regions of interest (highlighted in figure 34 picture d) was normalized versus the background and plotted (figure 34 picture b). The first cycle of induction appears to be stronger and it is not clear if the decrease of fluorescence is due to the lower dosage or to a lower efficiency of the system.

\subsection{Immunological reactions}

A stereotaxic injection of a virus can activate the immune system in three ways: a) there might be a mechanical lesion due to the injection capillary, b) there might be an immunological reaction against the injected virus by itself or c) against the protein expressed after the transduction (pSwitch protein, EGFP, GDNF).

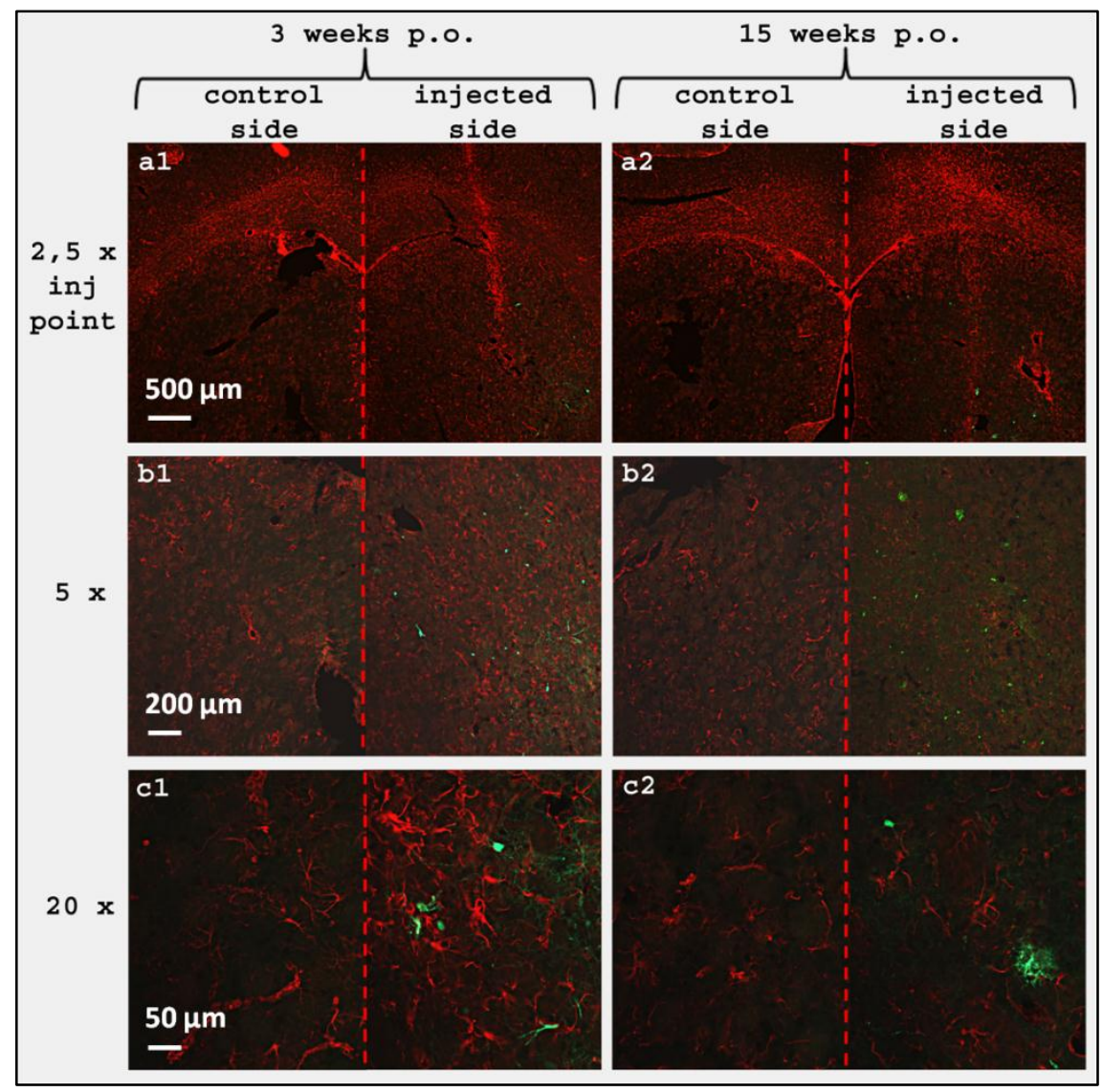

Figure 35) GFAP staining of injected brains. The scar formation due to the needle tract can be observed in the $2.5 x$ pictures of animals perfused 3 weeks after surgery (panel a1) and 15 weeks after surgery (panel a2). Astroglyosis is evident in the 5x (panel b1) and 20x (panel c1) pictures taken 3 weeks post operation while it is absent in animals sacrificed 15 weeks after surgery (panel b2 and c2). 
To address these issues, two immunostainings were performed; a GFAP staining to label astrocytes and an Iba1 staining to visualize microglial cells. Two time-points were investigated: the first one, corresponding to the first MF induction 3 weeks after surgery, and a second one, corresponding to the third MF induction performed approximately three months after surgery.

As can be seen in figure 35 panel a1 and a2 the injection tract is clearly visible at both time-points investigated. Astrocytes accumulate in the lesioned region giving rise to a scar that is persistent for at least 15 weeks post operation.

Panels $b$ and $c$ show an area 250-500 $\mu \mathrm{m}$ away from the point of injection where the virus is still able to infect cells but the effect of the injection scar should be less visible. Panel b1 shows that astroglyosis affects the whole striatum three weeks after surgery but the astroglyosis is no more present three months later (figure 35 panel b2).

In the Iba1 staining (figure 36) no difference can be seen between the injected side and the contralateral side in either the number or shape of the microglia cells suggesting that they do not become activated upon AAV-infection. No difference in the two time-points investigated was observed.

Taken together these results suggest that the immune system is activated only due to the mechanical lesion caused by the needle but the virus and the pSwitch protein appear not to be immunogenic in the rodent brain.

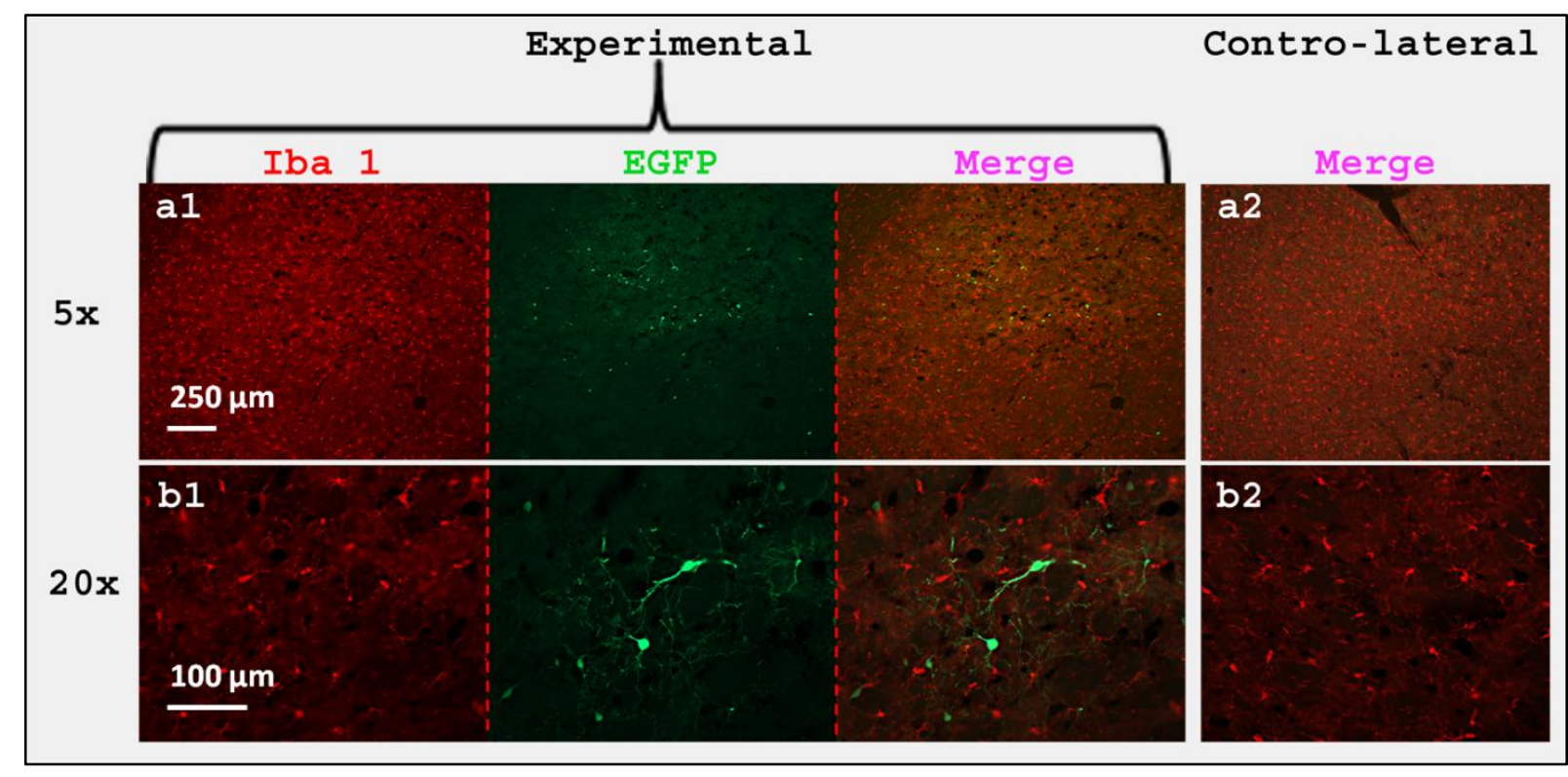

Figure 36) Iba1 staining of injected striatum. No difference in number and shape of microglial cells can be observed between the injected side (panels a1 and b1) and the contralateral side (pictures a2 and b2)

\section{7 "Leakiness" of the pSwitch system in the not induced state}

The EGFP expression in the off-state using AAV5 serotype viruses, the 3 transcription blockers, and omitting the WPRE, appears much lower compared to the first experiments performed, but it is not completely abolished.

This can be observed when pictures are taken with a long exposure time, high magnification or when the brightness and the contrast of the pictures are digitally modified to highlight this leakiness. 
Figure 37 shows pictures acquired and modified as mentioned. A low, but detectable, EGFP expression in the off-state can be seen in both experiments where the synapsin promoter and the Gfabc1d promoter were used to express the pSwitch protein in the inducing virus. The leakiness appears to be slightly higher in the case of the Gfabc1d promoter.

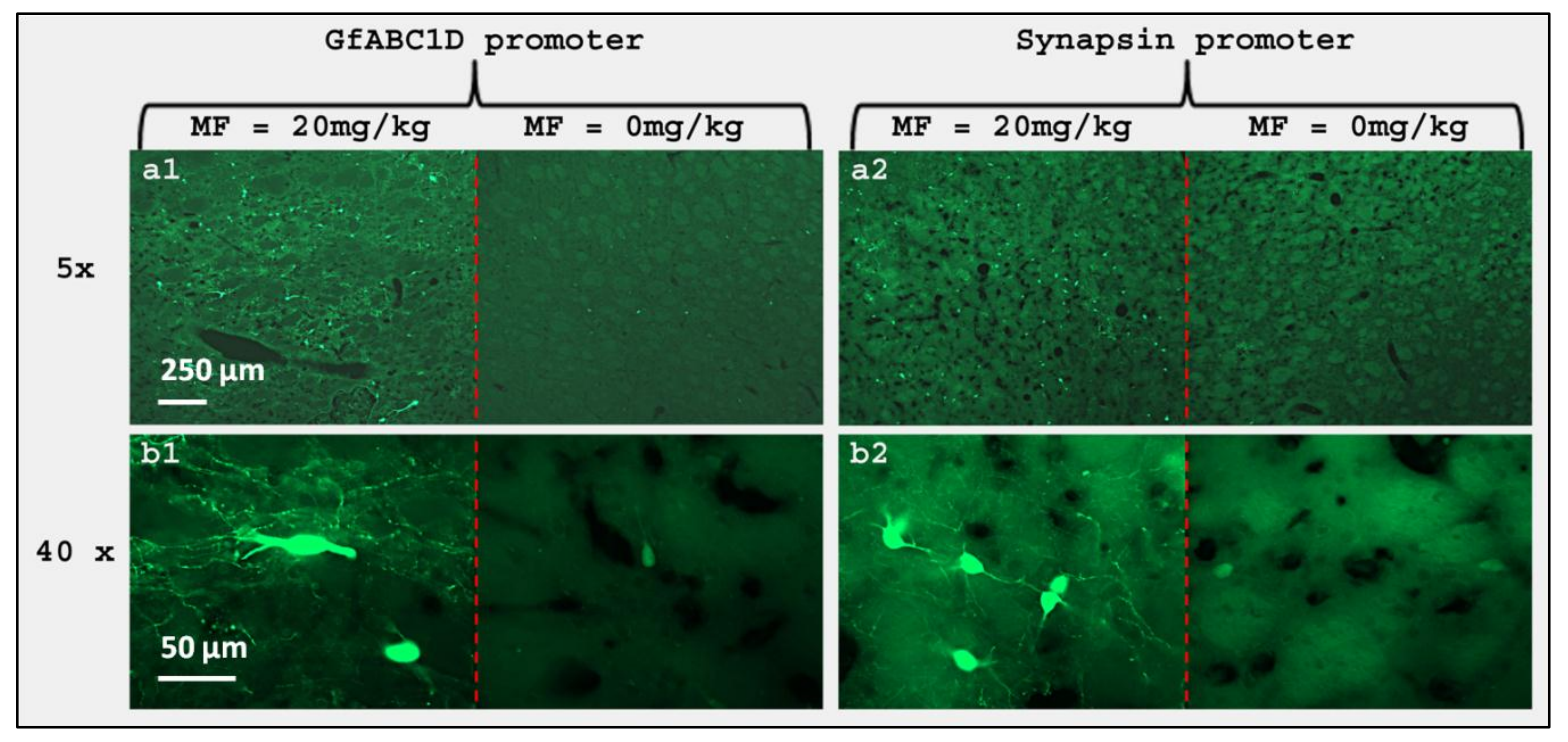

Figure 37) "Leakiness" of the pSwitch system. To highlight the EGFP expression in the off-state the $5 x$ pictures in panels a1 and a2 were taken with an exposure time of 30 seconds while the $40 x$ pictures in panels b1 and b2 were taken with an exposure time of 5 seconds. In both cases brightness and contrast were strongly increased. MF = mifepristone.

\subsection{Viral vectors interaction}

\subsubsection{Interaction between different serotypes (AAV5 and AAV6)}

Some tests were performed using AAV5 and 6 serotypes together in order to co-transduce the same area with both viruses. Previous works [113] claimed that AAV5 and 6 should not compete for the same receptors, but our results indicate a high competitiveness which leads to very poor co-infection of the same cells with the two different viruses. To demonstrate this observation, animals were injected with two combinations of viruses expressing DsRed and EGFP; AAV6 SDsRedWB (figure 10-c) + AAV6 SEWB (figure 10-a) and AAV6 SDsRed + AAV5 SEWB.

As shown in figure 38 when the same AAV6 serotype is used (figure 38 panel a) the infection of neurons is homogeneous and cells are equally transduced with both viruses. When two different serotypes (AAV5 and AAV6) were used (figure 38 panel b) a different pattern of infection could be observed; the DsRed expressing AAV6 virus infects neurons around the point of injection while the EGFP expressing AAV5 virus, spreads more and has lower affinity for the receptors, infects only neurons which are not reached by the AAV6 virus. 


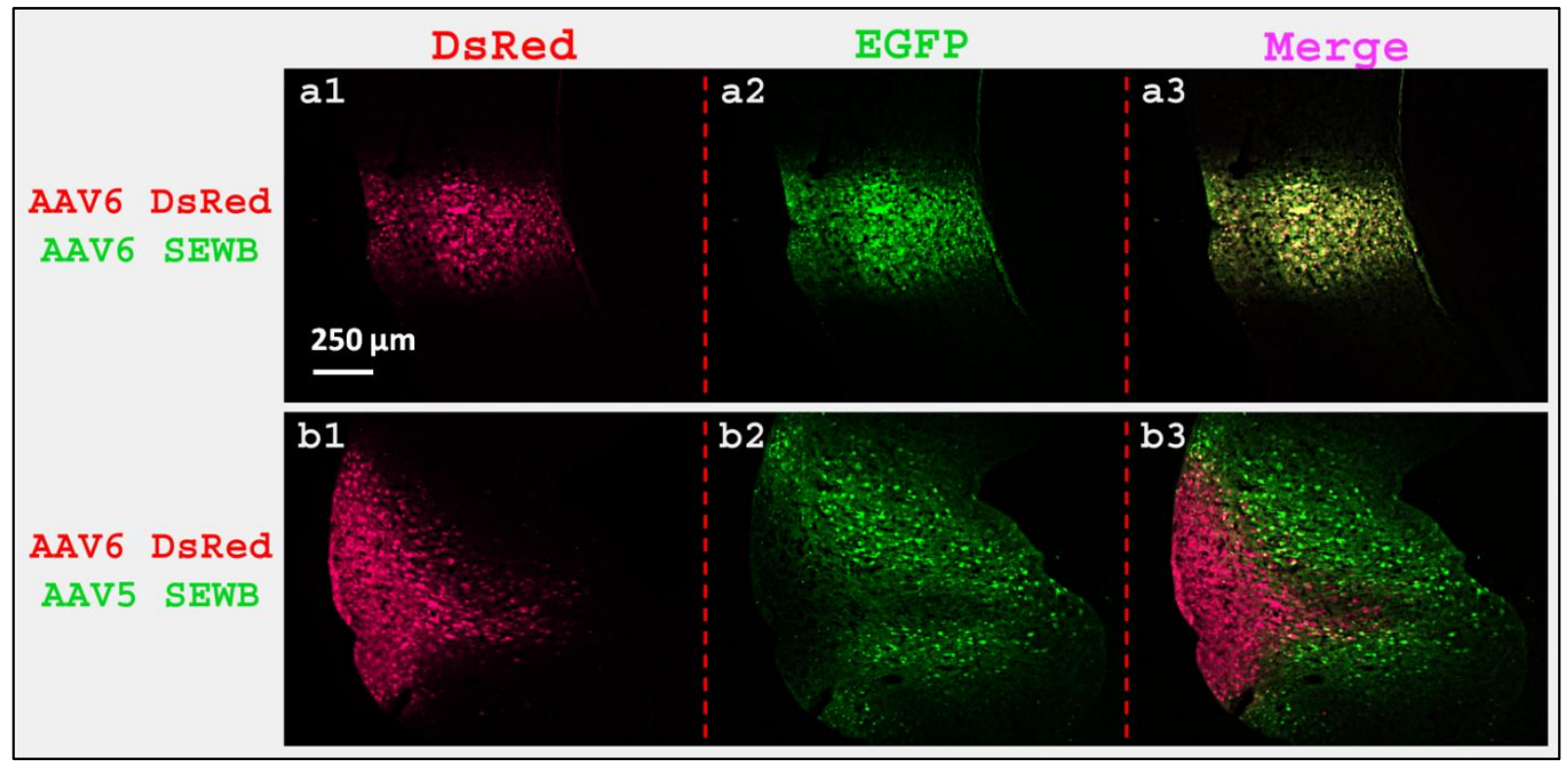

Figure 38) Transduction interference between AAV5 and AAV6 viruses. DsRed (pictures a1 and b1), EGFP (pictures a2 and b2) and merge (pictures a3 and b3) pictures of mice cortex injected with $1 * 10^{8}$ AAV6 SDsRed $+1 * 10^{8}$ AAV6 SEWB (panel a) and with $1 * 10^{8}$ AAV6 SDsRed $+1 * 10^{8}$ AAV5 SEWB (panel b). When the same serotype is used the infection is overlapping (picture a3), while, when different serotypes are used, the infection is selective (picture b3) and the virus with higher affinity for the cell receptors (AAV6) does not allow the virus with lower affinity (AAV5) to enter into the cells. AAV5, able to spread more, infects cell around the AAV6 area.

\subsubsection{Interaction between viruses of the same serotype (AAV5) and different promoters}

The previous experiment showed that the use of two viruses with the same serotype seems not to interfere with the pattern of infection. As AAV viruses have a low DNA packaging capacity, cotransduction with two AAV viruses have been routinely used [114-117]. With this assumption the experiment for multiple induction (chapter 4.5.1 "Multiple induction in rat striatum") was first performed using a third virus expressing constitutively the fluorescent protein DsRed under control of the synapsin promoter; this third virus was meant to be a reference for finding the point of injection and a control for comparing the pSwitch system between different animals. This experiment revealed that the addition of a third virus changed the pattern of infection and activation of the pSwitch system.

Figure 39 panel a shows the infection pattern of the pSwitch system together with the Dsred virus while in figure 39 panel $b$ shows the infection pattern when only the two viruses of the pSwitch system were used. In the first case a decrease of the EGFP expression and in the number of EGFP expressing cells can be clearly seen indicating interference in the infection or in the transgene expression between the pSwitch system viruses and the DsRed control virus. This decrease in efficiency might be due to the competitiveness for the cell viral receptors, rearrangement of the viral genome upon transduction, or to competitiveness for the cell transcription machinery due to the different activity of the promoters. 


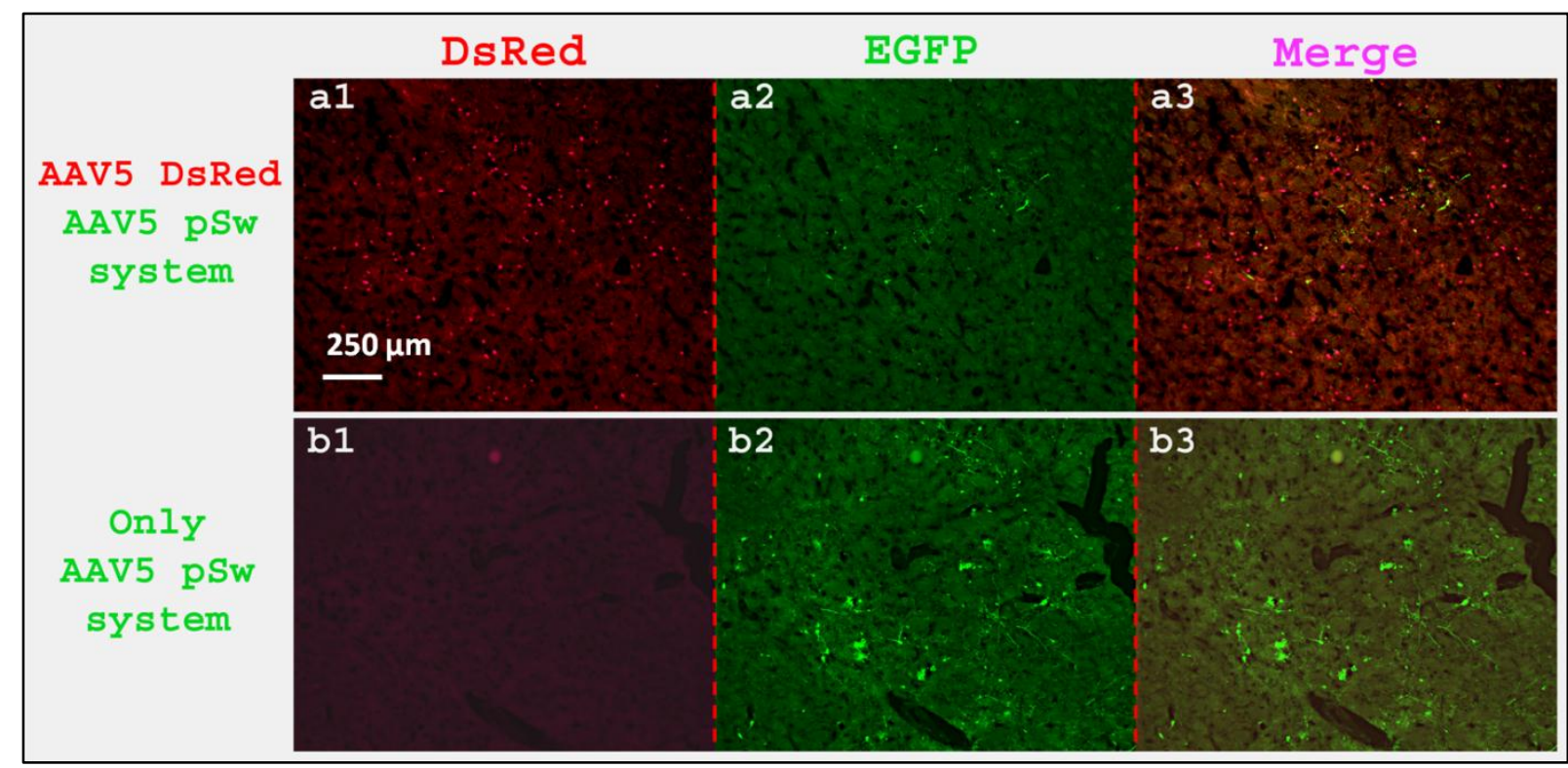

Figure 39) Transduction interference between AAV5 SdsRed and AAV5 pSwitch system. DsRed (pictures a1 and b1), EGFP (pictures a2 and b2) and merge (pictures a3 and b3) pictures of rat striatum injected with $5 * 10^{7}$ AAV5 SDsRed $+3 * 10^{7}$ AAV5 GfABC1DpSwS $+1 * 10^{8}$ AAV5 3TB UTEB (panel a) and 3*10 $0^{7}$ AAV5 GfABC1DpSwS $+1 * 10^{8}$ AAV5 3TB UTEB (panel b). The transduction efficiency appears much lower when the DsRed expressing virus is present.

\subsection{6-OHDA lesion paradigm}

In order to evaluate if the pSwitch system might be suitable for neurotrophic factor expression in Parkinson's disease, there is a need for a rat model of the disease with a partial loss of the dopaminergic system in the striatum. The lesion has to be strong enough to show motor deficits that can be evaluated by appropriate motor tests but not too harsh, in order to allow the neurotrophic factor to act on surviving cells. To generate the lesion, the dopaminergic neuron's selective toxin 6hydroxydopamine (6-OHDA) was used. Motor tests used in this experiment were the cylinder test and the apomorphine induced rotation.

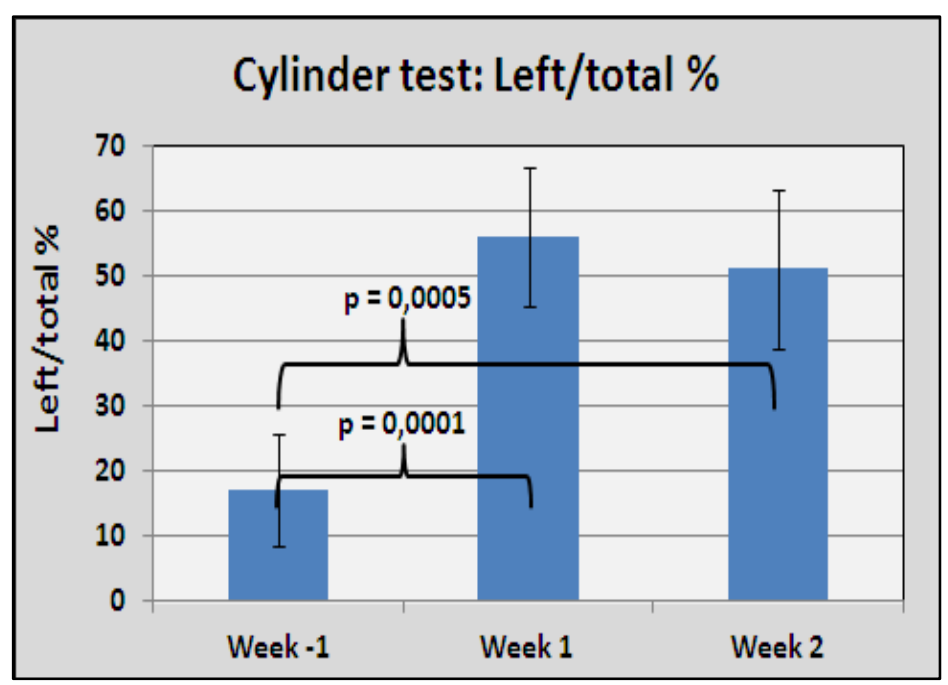

Figure 40) Cylinder test before and after the 6OHDA lesion. Data are presented as left/total touches. Before the operation (week -1) animals use only the left paw approximately $20 \%$ of the times as expected. After the lesion (week 1 and 2) animals use exclusively the left paw approximately $50 \%$ of the times indicating a partial lesion of the dopaminergic system in the left striatum. Error bars $=$ standard deviation. $p=2$ tailed $t$ test. 
The study for the right injection coordinates and proper amount of injected toxin was previously performed by Dr. Yuliya Tereschenko. A total of $10 \mu \mathrm{g}$ of $6-\mathrm{OHDA}$, diluted in $4 \mu \mathrm{l}$ of PBS $0.2 \%$ ascorbic acid, were injected in two different points $(2 \mu \mathrm{l} /$ injection) with the following coordinates: bregma +0.05 , lateral +0.21 , depth between -0.5 and -0.6 (depending on the weight), and bregma -0.05 , lateral +0.38 , depth between -0.5 and -0.6 (depending on the weight).

Animals, which were tested for the two motor tests before surgery, were injected with the toxin and tested again twice, one and two weeks post surgery. Rats were then sacrificed and the striata were cut and stained with the dopaminergic neurons marker TH.

In figure 40 data obtained from the cylinder test in which the results are presented as left/total touches are reported; before the operation animals used their paws normally (the left one approx. $20 \%$ of the total times) but after the lesion there was a significant increase in the preferential use of the left paw to approximately $50 \%$ (or a decrease in the use of the right paw) indicating a dysfunction in the left striatum. The fact that animals are not exclusively using the left paw indicates that the lesion of the dopaminergic system is partial and not total. No difference in the behavior can be observed between the first and the second test after the lesion.

The results obtained from the apomorphine induced rotation test are presented in figure 41 in which the observed data are plotted in total turns/hour. The experiment was repeated twice (week 1 and 2)

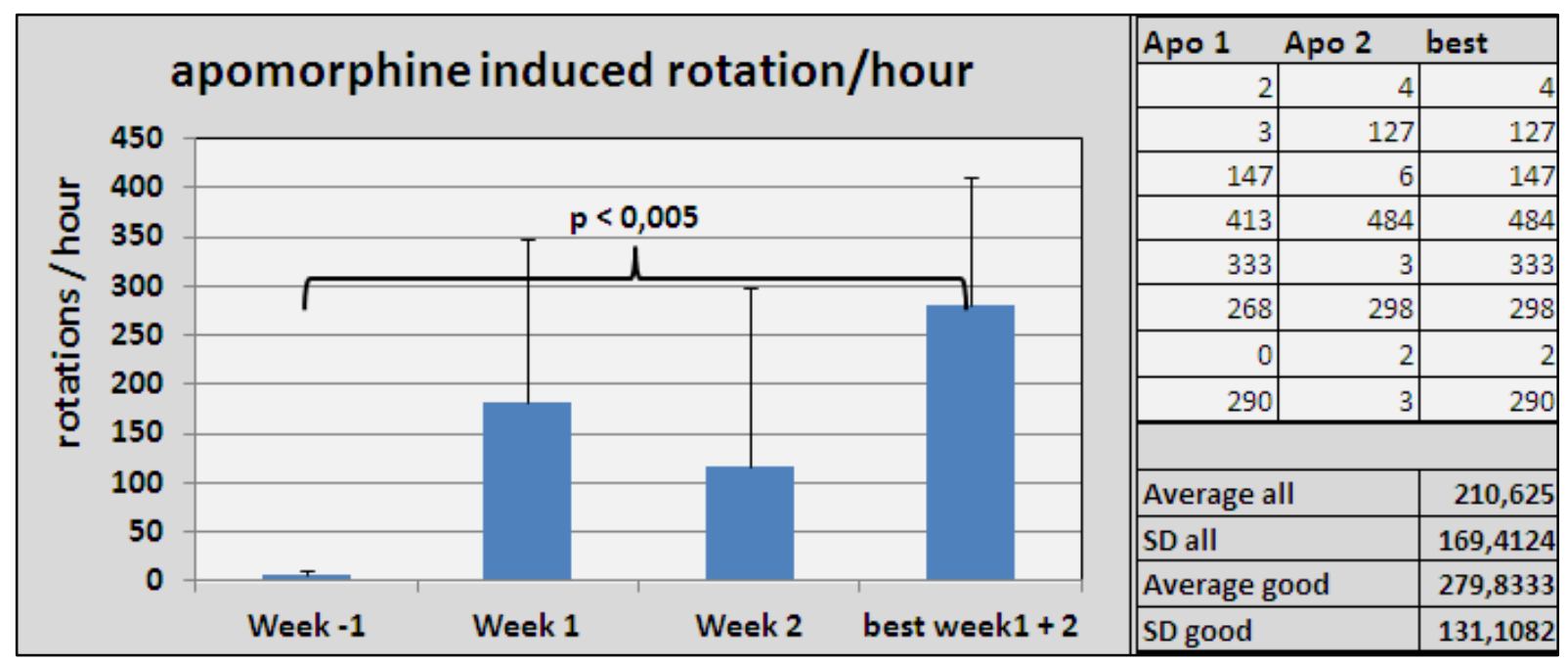

Figure 41) Apomorphine induced rotations before and after 6-OHDA lesion. Results are presented as total turns/hour. In the table are reported the results for every single animal in each session; as the variability was high we decided to consider the average of only the best performance out of the two ("average all") and then to exclude animals that did not perform in both tests ("average good"). As shown in the graph the increase in rotation becomes significant only when this method is used. Error bars = standard deviation. $S D=$ standard deviation. $p=2$ tailed $t$ test.

but, as can be seen from the table in figure 41 in which the results of every animal are presented separately, the test shows a huge variability between animals and in assessing the same animal consecutively; indeed some rats never respond to the drug, some respond only one time and some respond both times. To overcome this problem, only the best performance of the two tests was considered and animals that displayed less than 50 rounds/hour were not used for further analysis. By examining data in this way we can see a significant increase in the rotations of animals preoperation (not rotating, week -1 ) and post operation (approx 280 rotations/hour; best week $1+2$ ). A number of rotations between 200 and 300 indicates a partial lesion. This data should be considered carefully due to the large variability that they show. 
After the test animals were sacrificed and their brains were cut and stained for the dopaminergic neuron marker $\mathrm{TH}$.

As can be seen from figure 42 the contralateral striatum (panel a) appears intact while the lesion in the left striatum (panel b) is evident and appears as a lack of TH staining in the striatum. Nevertheless it is evident that not all the fibers were destroyed indicating a partial rather than total lesion of the dopaminergic system.
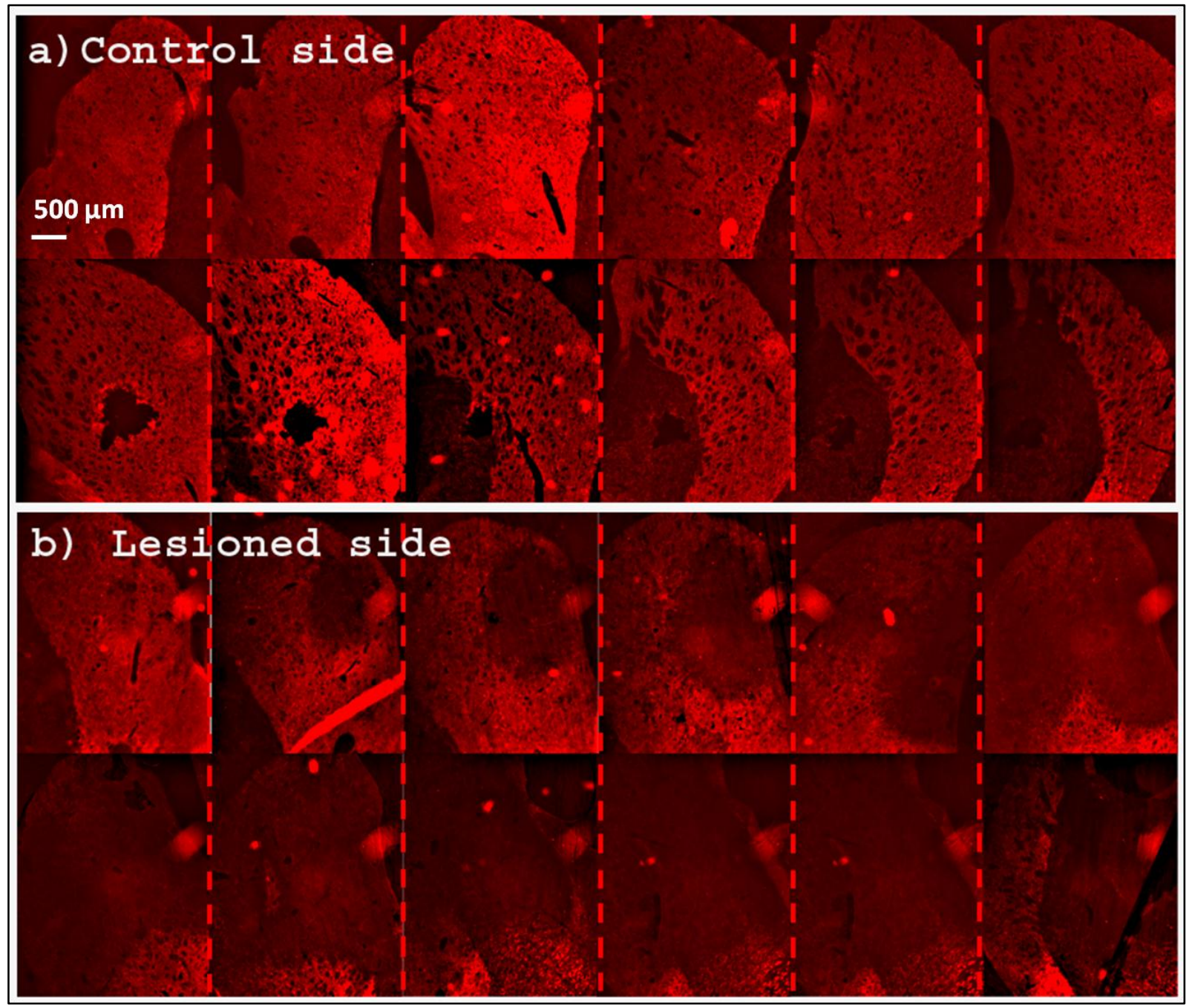

Figure 42) TH staining of control side (panel a) and lesioned side (panel b) of rats striatum injected with the dopaminergic neuron's toxin 6-OHDA. The striatum in the control side appears intact while the lesioned side shows a lack of TH staining indicating a loss of $\mathrm{TH}$ positive fibers. $\mathrm{TH}=$ tyrosine hydroxylase.

\subsection{Regulated GDNF expression in 6-0HDA lesioned rats}

\subsubsection{Layout of the experiment}

In order to evaluate if short term expression of GDNF, induced by MF, can restore a partial lesion of the dopaminergic system, EGFP was substituted in the responsive vector with GDNF.

As mentioned above, two parallel experiments were performed in which two different promoters were used to express the pSwitch protein in the inducing virus; the Gfabc1d (experiment performed by Dr. Yuliya Tereshchenko) and the synapsin promoter (performed in this study). In this section only experiments done with the synapsin promoter are presented. 
A total number of 107 animals were tested for three motor tests: apomorphine induced rotation, cylinder test, and corridor test. Animals that did not perform according to the behavior expected for healthy animals (no apomorphine induced rotation, $20 \pm 10 \%$ left/total paw touches in the cylinder test, and $50 \pm 20 \%$ left/ total food retrieval in the corridor test) were excluded from the experiment while the rest were injected with 6-OHDA as previously described. This time-point was considered to be week 0 .

Animals were tested again for the three motor tests and animals that showed a too harsh or a too mild lesion were excluded. The acceptable range was decided by having at least two out of three tests in the following ranges: between 200 and 350 rotations in the apomorphine induced rotation test; $50 \% \pm 20 \%$ Left / total paw touches in the cylinder test; and $80 \% \pm 20 \%$ Left / total food retrieval in the corridor test.

On week 2 animals that fulfilled previous criteria were divided into the following 4 groups and a double injection at the same coordinates of the 6-OHDA deposits was performed:

a. AAV5 SpSwS $3 * 10^{7}+$ AAV5 UT-GDNF-B $1 * 10^{8}$ with MF induction as "treated" animals (24 animals)

b. AAV5 SpSwS $3 * 10^{7}+$ AAV5 UT-GDNF-B $1 * 10^{8}$ without MF induction as "not treated" animals (22 animals)

c. AAV5 S-GDNF-B $1 * 10^{8}$ as a positive control ( 24 animals)

d. AAV5 SEWB $1 * 10^{8}$ as a negative control (21 animals)

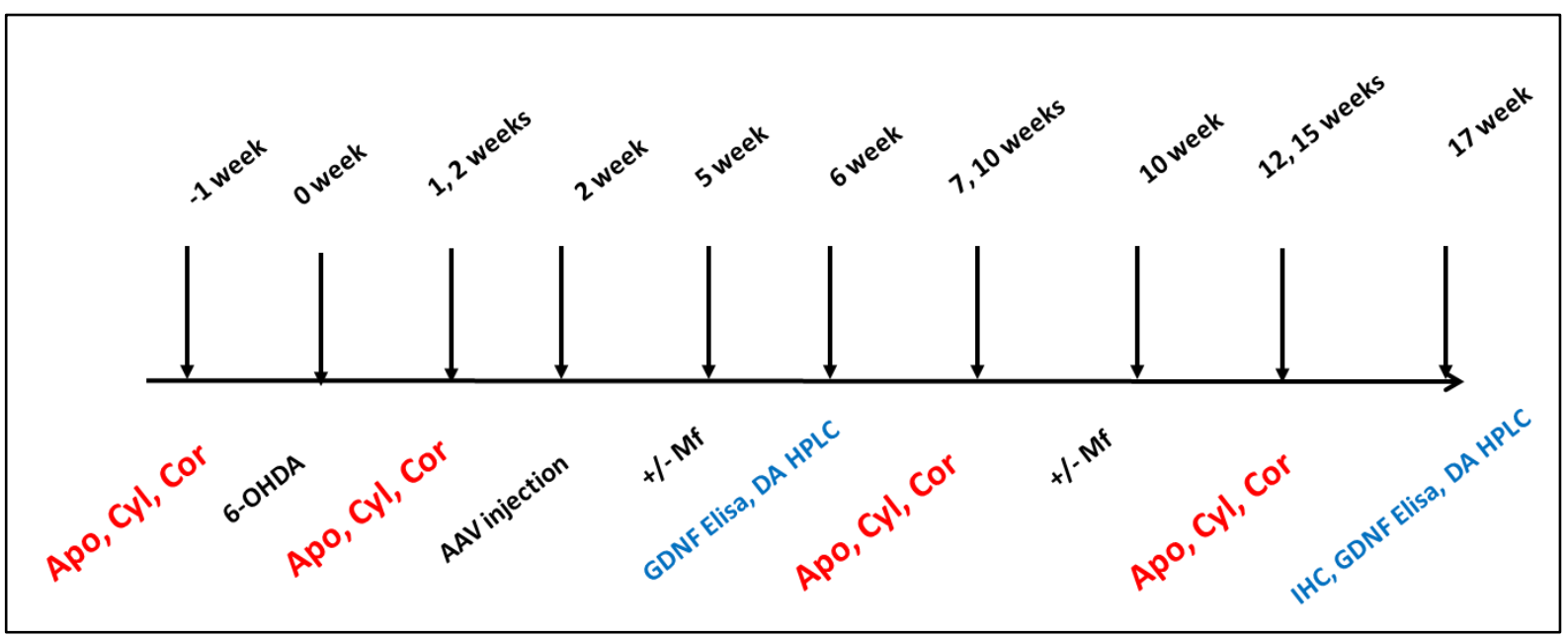

Figure 43) Time-table of the 6-OHDA lesion and restoration experiment. Animals were tested for the motor tests before the operation (week -1). Rats were then injected with 6-OHDA (week 0), tested again (week 1-2) and injected with the 4 different combinations of viruses (week 2). Half of the animals which received the pSwitch system were injected with MF at week 5 and 10 while the other half received DMSO. Rats were tested for the three motor tests at week 7, 10, 12 and 15. Some of the animals in each group were sacrificed at week 6 and 17 in order to measure GDNF level by ELISA and catecholamines concentration by HPLC. Leftover animals were sacrificed and perfused at week 17 for histological analysis. $\mathrm{Apo}=$ apomorphine induced rotation; $\mathrm{Cyl}=$ cylinder test; $\mathrm{Cor}=$ corridor test; $6-\mathrm{OHDA}=6-\mathrm{OHDA}$ lesion; $\mathrm{DA}=$ dopamine.

Animals were left to recover for 3 weeks and on week 5 group a received a first cycle of MF induction while group b received only the solvent DMSO.

At week 6 seven animals from group $b$ and $d$, and 8 animals from group a and $c$ were sacrificed in order to measure the level of GDNF by ELISA and the amount of Dopamine, DOPAC and HVA by HPLC. 
Between week 7 and 10 animals were tested twice for the three motor tests; the apomorphine induced rotation test was repeated two times each time and only the higher number of rotation was considered.

At week 10 group a received a second MF induction and group $\mathrm{b}$ received again DMSO.

Between week 12 and 15 animals were tested twice for the three motor tests; the apomorphine induced rotation test was repeated two times each time and only the higher number of rotation was considered.

At week 17 six animals per group were sacrificed for GDNF and dopamine measurement while the residual rats were perfused in order to cut and stain the brains for histological analysis.

In this chapter each test is presented and discussed separately.

\subsubsection{Apomorphine induced rotation test}

For the apomorphine induced rotation test, animals were injected intraperitoneally with $4 \mathrm{mg} / \mathrm{kg}$ of apomorphine diluted in saline solution and were placed in a $30 \mathrm{~cm}$ diameter cylinder; the total $360^{\circ}$ rotation in one hour were counted.

As shown in figure 44 animals were not rotating before the 6-OHDA lesion, while in week 1-2 they displayed between 200 and 320 rotations/hour. In the following time points, all groups decreased the number of rotations without a significance difference between the 4 groups indicating a possible endogenous recovery of the dopaminergic system. In all cases a huge standard deviation can be observed which invalidate the significance (except between the first 2 time-points).

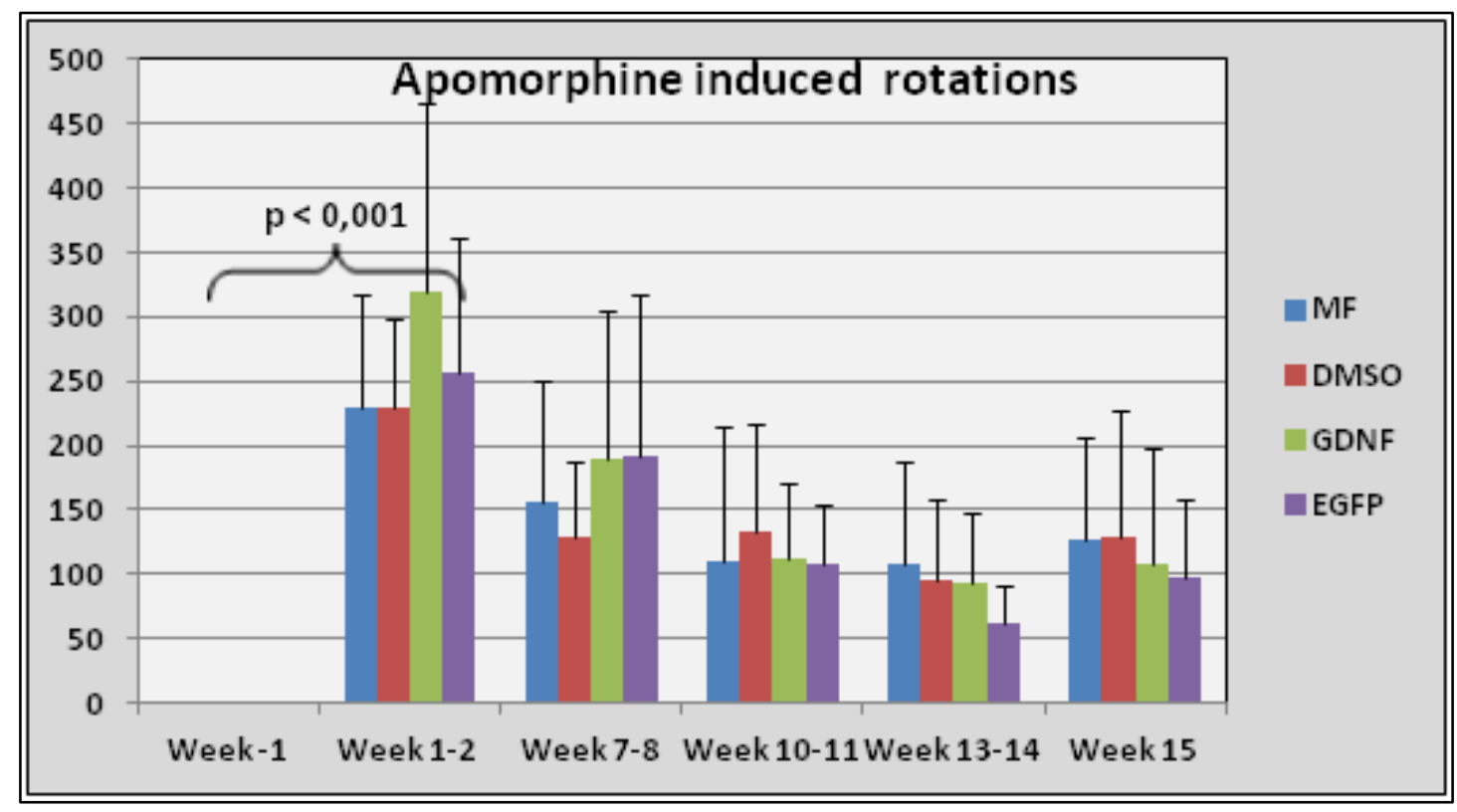

Figure 44) Apomorphine induced rotation presented in $360^{\circ}$ total turns/hour. Blue bars refer to animals injected with the pSwitch system and induced with MF; red bars refer to animals injected with the pSwitch system and injected with DMSO; Green bars refer to animals injected with the virus expressing GDNF constitutively; Violet bar refers to animals injected with a EGFP expressing virus. Before 6-OHDA lesion animals do not rotate upon apomorphine injection while they all rotate between $\mathbf{2 2 0}$ and $\mathbf{3 2 0}$ turns/hour after the lesion. In the subsequent tests all groups show a decrease in rotation without any differences between groups. Error bars = standard deviation. $p=2$ tailed t test. 


\subsubsection{Corridor test}

For the corridor test animals were first trained by placing them in the corridor with free available corn flakes at least three times before the first test and one time before each of the following tests.

As can be deduced from the graph in figure 45 , at week -1 animals behaved normally, retrieving the food equally from both sides. On week 1 , after the lesion, animals retrieved the food preferentially from the left side (around $80 \%$ ). In the following time-points we can observe a decrease of the average retrieval to $70 \%$ left and $30 \%$ right without any significance difference between the groups. A big standard deviation can be observed which invalidate the significance except for the first 2 time points considered.

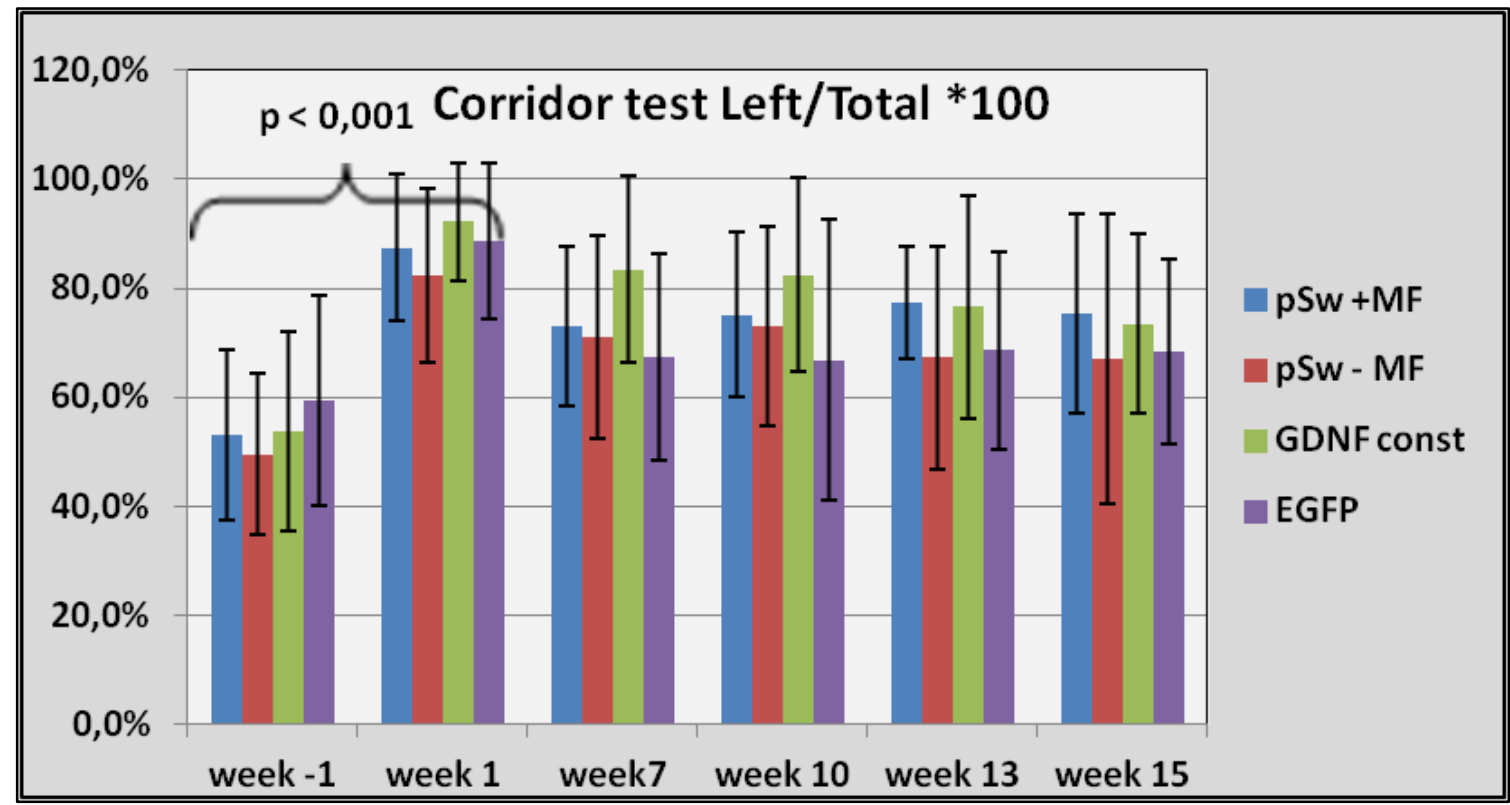

Figure 45) Corridor test; here data are presented as left/total food retrieval. Blue bars refer to animals injected with the pSwitch system and induced with MF; red bars refer to animals injected with the pSwitch system and injected with DMSO; Green bars refer to animals injected with the virus expressing GDNF constitutively; Violet bar refers to animals injected with a EGFP expressing virus. Before 6-OHDA lesion animals retrieved the food $50 \%$ from both side while after the lesion they retrieved food more than $80 \%$ from the left side, indicating a partial lesion of the left dopaminergic system. Error bars $=$ standard deviation. $p=2$ tailed $t$ test.

\subsubsection{Cylinder test}

In the cylinder test the animal is placed in a transparent cylinder of $24 X 40 \mathrm{~cm}$ (diameter X height) for $10 \mathrm{~min}$ and recorded; the video is then analyzed and the number of times the animal climb the wall using only his left, only his right or both front paws is counted. The results are then plotted as left/total touches * 100 .

In the graph in figure 46 , before the operations, at week -1 , animals climbed the wall by using only the left paw $20 \%$ of the times as expected. 
After the 6-OHDA lesion it is demonstrated an increase in the use of only the left paw to approximately $50 \%$ over the total touches; in the following tests a partial recovery in all groups is observed without significant differences between them. The large standard deviation invalidates the significance except for the first 2 time points considered.

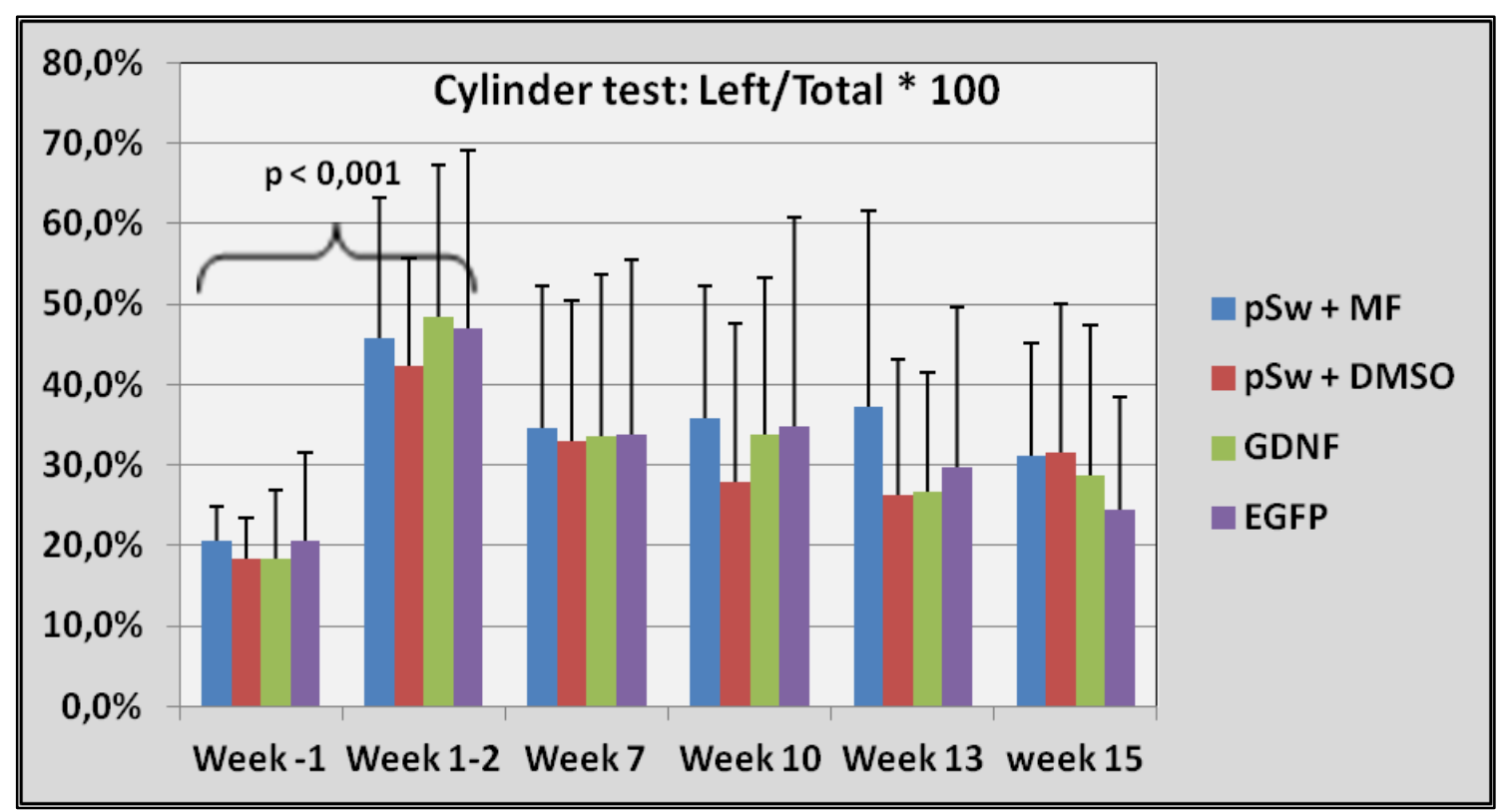

Figure 46) Cylinder test; here data are presented as left/total touches. Blue bars refer to animals injected with the pSwitch system and induced with MF; red bars refer to animals injected with the pSwitch system and injected with DMSO; Green bars refer to animals injected with the virus expressing GDNF constitutively; Violet bar refers to animals injected with a EGFP expressing virus. Before 6-OHDA lesion animals were climbing the wall $20 \%$ exclusively with the left paw while after the lesion they climbed $\sim 50 \%$ only with the left paw, indicating a partial lesion of the left dopaminergic system. Error bars $=$ standard deviation. $p=2$ tailed $t$ test.

\subsubsection{Dopamine level by HPLC}

At week 6 and 17 animals were sacrificed and part of the striata were treated in order to measure the amount of dopamine, DOPAC and HVA.

As shown in the graphs in figure 47 we can observe a decrease of dopamine in the left striatum, the one that was lesioned, comparing with the contralateral side. At week 17 the amount of dopamine was further decreased compared to the previous time point without any significance difference in the different groups. 


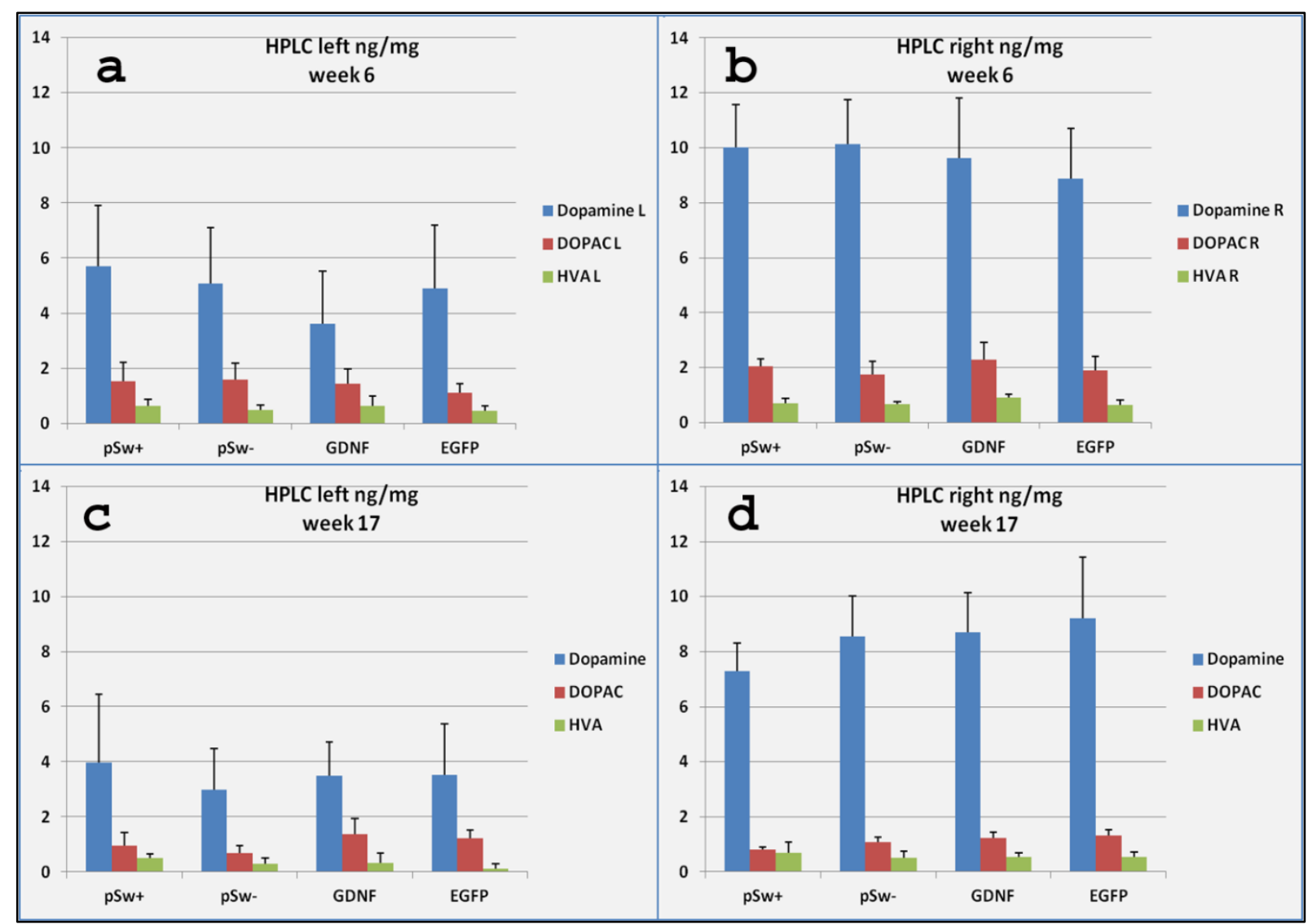

Figure 47) Total dopamine, DOPAC and HVA measured in rat striatum in $\mathrm{ng} / \mathrm{mg}$ tissue. a) left striatum measurement at week 6; b) contralateral right striatum dopamine content at week 6; c) left striatum measurement at week 17; d) contralateral right striatum dopamine content at week 17. Total dopamine content in the left striatum is decreased comparing to the right striatum in all groups at week 6 after the 6-OHDA lesion. No recovery is observed in any group at week 17 where the total dopamine content in the left striatum which stays at a level approximately of $50 \%$ compared to the right striatum. pSw+ = animals injected with AAV5 SpSwS + AAV5 3TB-UT-GDNF-B induced with MF; pSw- = animals injected with AAV5 SpSwS + AAV5 3TB-UT-GDNF-B injected with DMSO; GDNF = animals injected with AAV5 S-GDNF WB (virus expressing GDNF constitutively under the synapsin promoter); EGFP = animals injected with AAV5 SEWB (virus expressing EGFP constitutively under the synapsin promoter). Each group $n=6$. Error bars $=$ standard deviation.

\subsubsection{GDNF ELISA}

The same brains used for dopamine evaluation were used for GDNF measurement by ELISA. From the data reported in table 7, which refers to the measurements done on animals sacrificed at week 6 , and table $b$, which refers to animals sacrificed at week 17 we can draw the following conclusions: a) there is a variability in endogenous GDNF expression (measured in the right striatum) but the concentration is always between 1 and $4.3 \mathrm{pg} / \mathrm{mg}$ tissue; $\mathrm{b}$ ) an increase in the GDNF level is observed at week 6 when the system is activated ( $54 \mathrm{pg} / \mathrm{mg}$ tissue); c) GDNF level in induced animals decreases to basal level at week 17; d) the basal level of expression of GDNF in animals injected with the pSwitch system is always double that of the contralateral side; e) the GDNF concentration in brains injected with the NF constitutively expressing virus was much higher than in experimental animals and decreases at week 17; f) no effects on GDNF level can be observed in animals injected with the EGFP expressing virus. 


\begin{tabular}{|l|ll|ll|}
\hline & Week 6 & Week 17 & \\
\hline & $\begin{array}{l}\text { GDNF left } \\
(\mathrm{pg} / \mathrm{mg})\end{array}$ & $\begin{array}{l}\text { GDNF right } \\
(\mathrm{pg} / \mathrm{mg})\end{array}$ & $\begin{array}{l}\text { GDNF left } \\
(\mathrm{pg} / \mathrm{mg})\end{array}$ & $\begin{array}{l}\text { GDNF right } \\
(\mathrm{pg} / \mathrm{mg})\end{array}$ \\
\hline $\mathrm{pSw+}$ & $\mathbf{5 4 . 3 9}$ & $\mathbf{2 . 9 4}$ & $\mathbf{8 . 5 2}$ & $\mathbf{3 . 9 9}$ \\
SD & 44.94 & 0.73 & 2.30 & 1.98 \\
$\mathrm{pSW}-$ & $\mathbf{6 . 2 5}$ & $\mathbf{3 . 2 2}$ & $\mathbf{2 . 7 7}$ & $\mathbf{1 . 1 9}$ \\
SD & 2.80 & 1.20 & 1.31 & 0.27 \\
GDNF & $\mathbf{2 1 3 8 . 6 8}$ & $\mathbf{3 . 8 5}$ & $\mathbf{1 4 9 7 . 1 8}$ & $\mathbf{4 . 1 0}$ \\
SD & 319.59 & 1.18 & 635.75 & 0.73 \\
EGFP & $\mathbf{4 . 1 2}$ & $\mathbf{4 . 3 1}$ & $\mathbf{3 . 0 4}$ & $\mathbf{3 . 7 1}$ \\
SD & 0.94 & 0.51 & 0.83 & 0.85 \\
\hline
\end{tabular}

Table 7) GDNF content in brain striatum measured by ELISA in $\mathrm{pg} / \mathrm{mg}$ tissue. $\mathrm{pSw}+=$ animals injected with AAV5 SpSwS + AAV5 3TB-UT-GDNF-B induced with MF; pSw- = animals injected with AAV5 SpSwS + AAV5 3TB-UT-GDNF-B injected with DMSO; GDNF = animals injected with AAV5 S-GDNF WB (virus expressing GDNF constitutively under the synapsin promoter); EGFP = animals injected with AAV5 SEWB (virus expressing EGFP constitutively under the synapsin promoter); SD = standard deviation. Each group $n=6$.

\subsubsection{Immunohistochemistry}

In order to evaluate the size of the lesion and a possible recovery several rats was perfused and brains were cut and stained for the dopaminergic neuronal marker TH. The big variability observed in the motor behavioral tests was not observed in the IHC. Figure 48 shows pictures acquired from the brains of two animals from the group injected with the pSwitch system and induced with MF. The two graphs present the results obtained from the motor tests in which the apomorphine test is normalized to " 1 " with rotations measured after the lesion (week 2). The animal in panel a does not display any amelioration over time while the animal in panel $b$ shows a progressive recovery over time. This difference cannot be clearly seen in brain sections, where the TH staining appears to be similar in the two brains.

Further analysis is needed to evaluate if different areas of the brain and the different behaviors in the motor tests can be associated. 

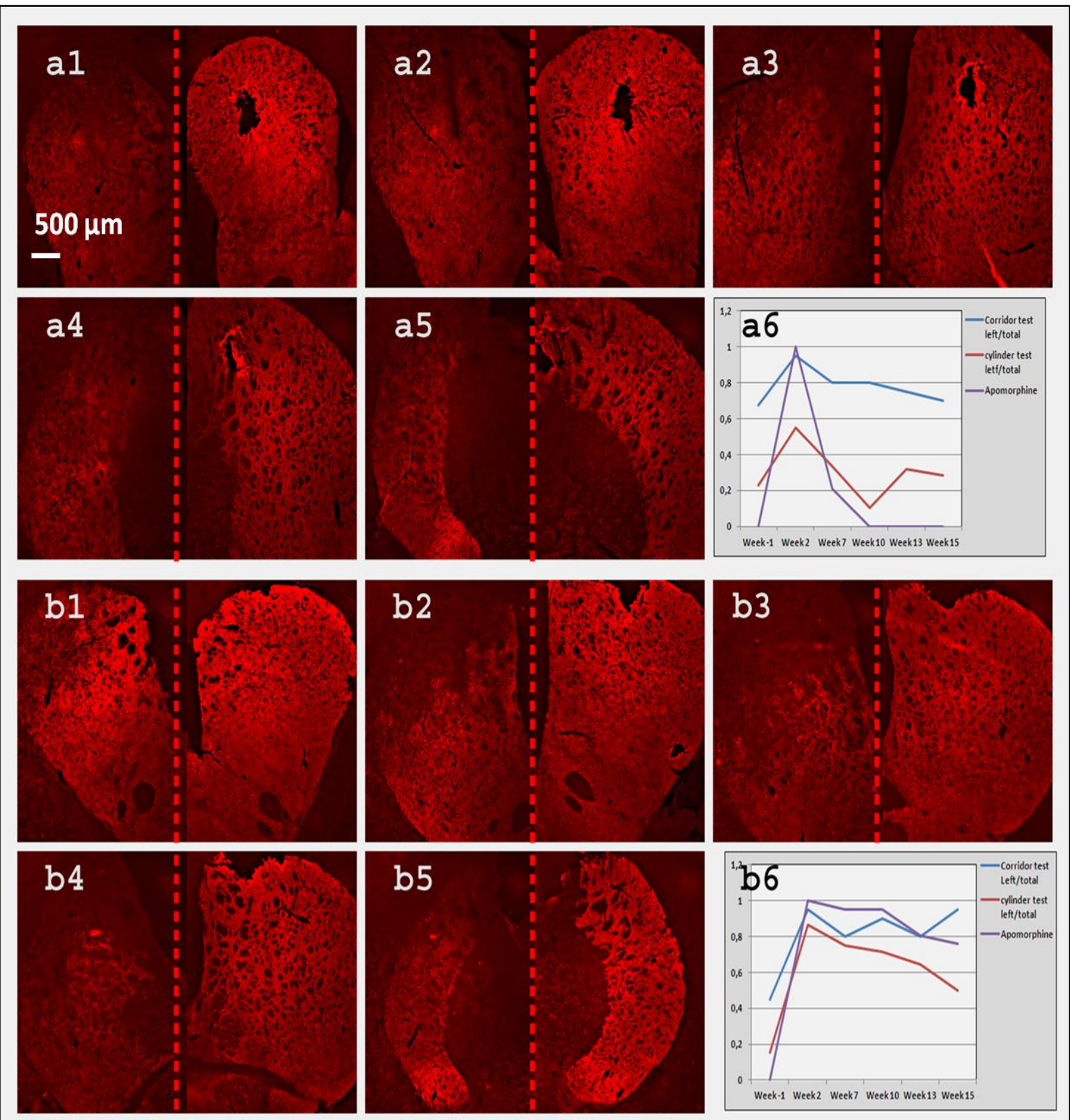

Figure 48) TH staining on striatum of two animals (panels a and b). Comparing panel a1-a5 (lesioned and contra-lateral in each panel) and b1-b5 the lesion appears to be comparable. In panel a6 and b6 results from motor tests are presented; blue = corridor test (left/total food retrieval); red = cylinder test (left/total touches; violet $=$ apomorphine induced rotation (rotation / rotation post lesion at week 2). Animal a show a recovery in the motor behavior with the values of the three tests that returns to pre-lesion value; animal b, contrarily, do not show significant amelioration and the values of the three tests remain at the post lesion level. 


\subsection{Dopamine production in cultures of cortical neurons}

Essential genes for dopamine synthesis are tyrosine hydroxylase (TH), which converts L-tyrosine to Ldopa, GTP cyclohydrolase 1 (GCH-1), which is the rate limiting enzyme for the synthesis of the TH essential co-factor tetrahydrobiopterine (BH4), aromatic amino acid decarboxylase (AADC), which converts L-dopa to dopamine, and vesicular monoamine transporter 2 (VMAT-2) which is essential for storing dopamine in vesicles. These genes, normally not expressed in cortical neurons, were cloned and packed in AAV6 viruses and used in different combination in primary cortical neurons culture in order to generate dopamine-producing cells.

Once a reproducible dopamine production was achieved, the interaction between $\alpha$-synuclein and dopamine was tested in order to assess a possible interaction between these two entities.

\subsubsection{Preliminary experiments}

Different trials had been carried out before being able to achieve a reproducible dopamine production from neurons. In this chapter a brief summary of these strategies is reported.

In the attempt to reduce the number of viruses needed for transduction, an IRES (Internal Ribosome Entry Site) element was used to express two genes from the same vector. Analysis performed using EGFP cloned downstream of IRES showed a really low efficiency of IRES (data not show) and this approach was abandoned.

A first attempt to produce dopamine from neurons involved a simultaneous transduction with $\mathrm{TH}$, AADC and VMAT-2; to promote the production of the catecholamine both the precursor L-Tyrosine and the essential co-factor BH4 were added but no dopamine was detected by HPLC.

The $\mathrm{BH} 4$ co-factor is unstable in culturing media and, in order to allow neurons to synthesize it, a virus expressing $\mathrm{GCH}-1$ was included in the experiment and dopamine production was promoted by adding L-Tyrosine but the trial was again unsuccessful (data not shown).

Finally a stable and reproducible dopamine production was achieved by transducing neurons only with AADC or with AADC + VMAT-2 and by adding in the culturing media the dopamine precursor Ldopa (figure 51).

\subsubsection{Protein expression upon viral transduction}

To verify gene expression neurons were infected with the different viruses, fixed with PFA and stained with the corresponding antibody.

As shown in figure 49 the TH and the AADC staining are specific and present only in infected neurons; concerning the VMAT-2 staining, a difference between infected and non infected neurons can be observed but the antibody used is clearly staining non-transduced neurons as well. Different antibodies and blocking procedures had been tested but a clearer staining could not be achieved indicating an unspecificyty of the antibody or an endogenous expression of VMAT-2 in nontransduced neuron. 


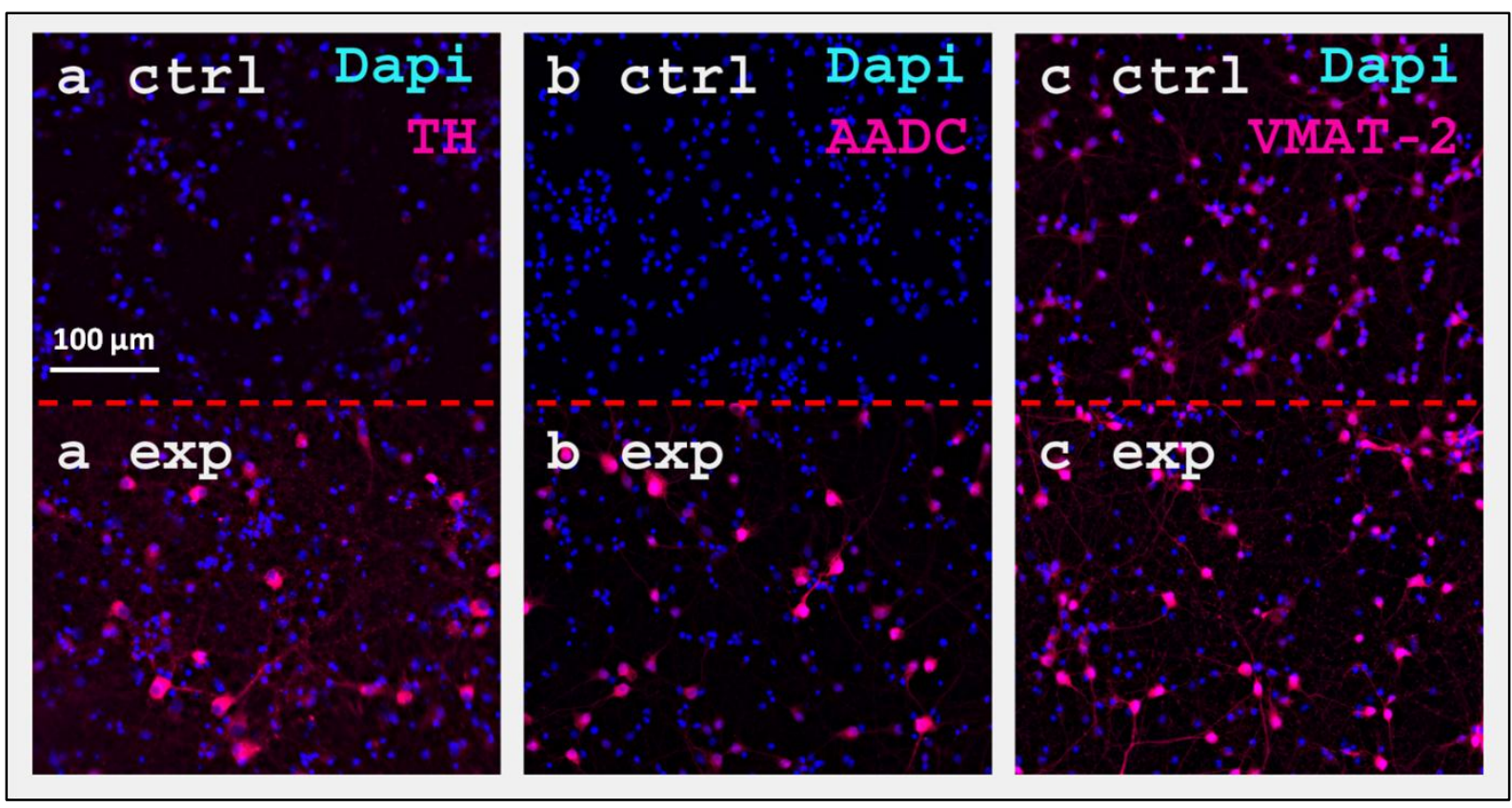

Figure 49) Pictures of IHC on dopamine producing neurons. Top pictures refer to non transduced control while bottom pictures refer to infected neurons. TH (picture a) and AADC (picture b) staining are specific while VMAT-2 staining (picture c) show staining both in the positive and negative control indicating or an unspecificity of the antibody or the presence of the protein endogenously. TH = tyrosine hydroxylase; AADC = aromatic amino acid decarboxylase; VMAT-2 = vesicular monoamine transporter 2 ; Ctrl = control.

For further confirmation of protein expression western blots on lysates of infected and controls neurons were performed. As shown in figure 50, a clear and specific band can be detected for TH while, in the case of AADC, a weak band is present in the negative control. Western blot for VMAT 2 showed only unspecific signals present both in experimental neurons and in negative control; different antibodies and blocking protocols were used but it was not possible to obtain a clearer result indicating, like in the IHC, or an unspecificity of the antibody or an endogenous expression of VMAT-2 in non transduced neurons.

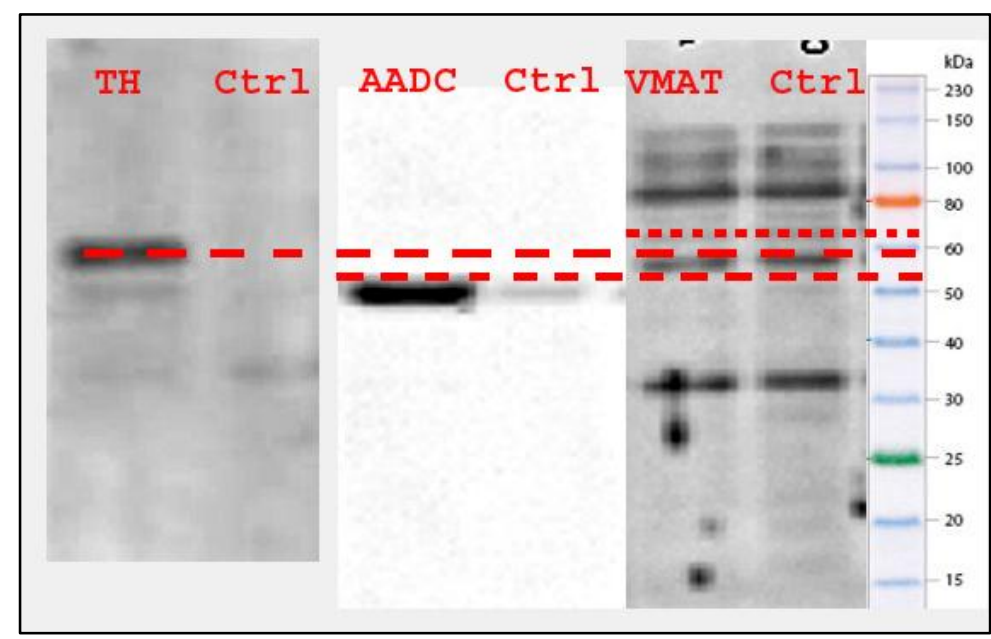

Figure 50) Western blot on neurons infected with viruses expressing proteins for DA production. TH and AADC staining are positive while VMAT-2 is unspecific. TH = tyrosine hydroxylase. AADC = aromatic amino acid decarboxylase. VMAT $=$ Vesicular monoamine transporter 2. Ctrl $=$ controls. Marker $=$ NEB ColorPlus Prestained Protein Marker, Broad Range (10 -230 kDa). 


\subsubsection{Dopamine level by HPLC}

A reproducible dopamine production from transduced primary cortical neurons could be achieved by infecting cells with viruses expressing AADC or AADC + VMAT-2 and using L-dopa to promote the production of the catecholamine. The amount of dopamine and its metabolites DOPAC and HVA was measured using an HPLC system coupled with an electrochemical detector. Both the released and intracellular stored fractions were assessed. Different parameters were evaluated in the experiments: I) presence/absence of VMAT-2, II) amount of viruses, III) amount and time of L-dopa incubation, and IV) culturing media. Four different groups were prepared in triplicate:

A) AADC + VMAT-2 high titre in RB (releasing buffer)

B) Only $A A D C$ high titre in $R B$

C) AADC + VMAT-2 low titre in RB

D) AADC + VMAT-2 high titre in HCN media

Releasing Buffer (RB) consists of a high potassium buffer that should favor the release of dopamine and protect it from degradation. HCN media was exchanged with RB before the L-dopa addition.

Influence of each parameter is show in the two graphs in figure 51 (intracellular fraction) and figure 52 (extracellular fraction). If not specified, data are considered significant when a 2 tailed $t$-Test was $<0.05$.

I. To evaluate the effect of VMAT-2 neurons were infected with both AADC and VMAT-2 or only with AADC. Both the internal fraction of dopamine (Figure 51 Box $A$ versus $B$ ) and the released one (figure 52 box $A$ versus $B$ ) are significantly higher in neurons expressing VMAT-2 when they were incubated with $10 \mu \mathrm{M}$ of L-dopa for 2 and 4 hours.

II. The influence on DA production depends significantly, for the $2 \mathrm{~h}$ incubation time, on the amount of used viruses; this is verified by comparing box $A\left(2.5^{*} 10^{7}\right.$ of each virus) and box $B$ $\left(1 * 10^{7}\right.$ of each virus) both in the intracellular fraction (figure 51$)$ and in the released fraction (figure 52). If the 4 hours incubation time is considered, a significant difference in favor of the neurons infected with the higher amount of viruses is obtained with a 1 tail t-Test indicating a tendency of higher production when higher amount of viruses are used.

III. In this experiment two different concentrations and two different incubation times of L-dopa were tested: 5 and $10 \mu \mathrm{M}$ concentration for $2 \mathrm{~h}$, and 2 and 4 hours incubation with $10 \mu \mathrm{M}$ concentration of L-dopa. No significant differences were observed for the intracellular fraction of the neurotransmitter (figure 51 box A, B, C, D) indicating a saturation of the system in all conditions. Contrarily, a significant difference in the released fraction of dopamine was observed depending on both time of incubation and amount of L-dopa used (figure $52 \mathrm{~A}, \mathrm{~B}, \mathrm{C}, \mathrm{D}$ ). The increase of both time of incubation and amount of L-dopa in the media lead to an increase of released dopamine. 
IV. The two different incubation media considered do not affect the intracellular storage capacity of dopamine (figure 51 Box A versus D) while, observing the released fraction of the neurotransmitter, we can notice a significant decrease in the release when neurons were incubated in HCN media (figure 52 Box A versus D).

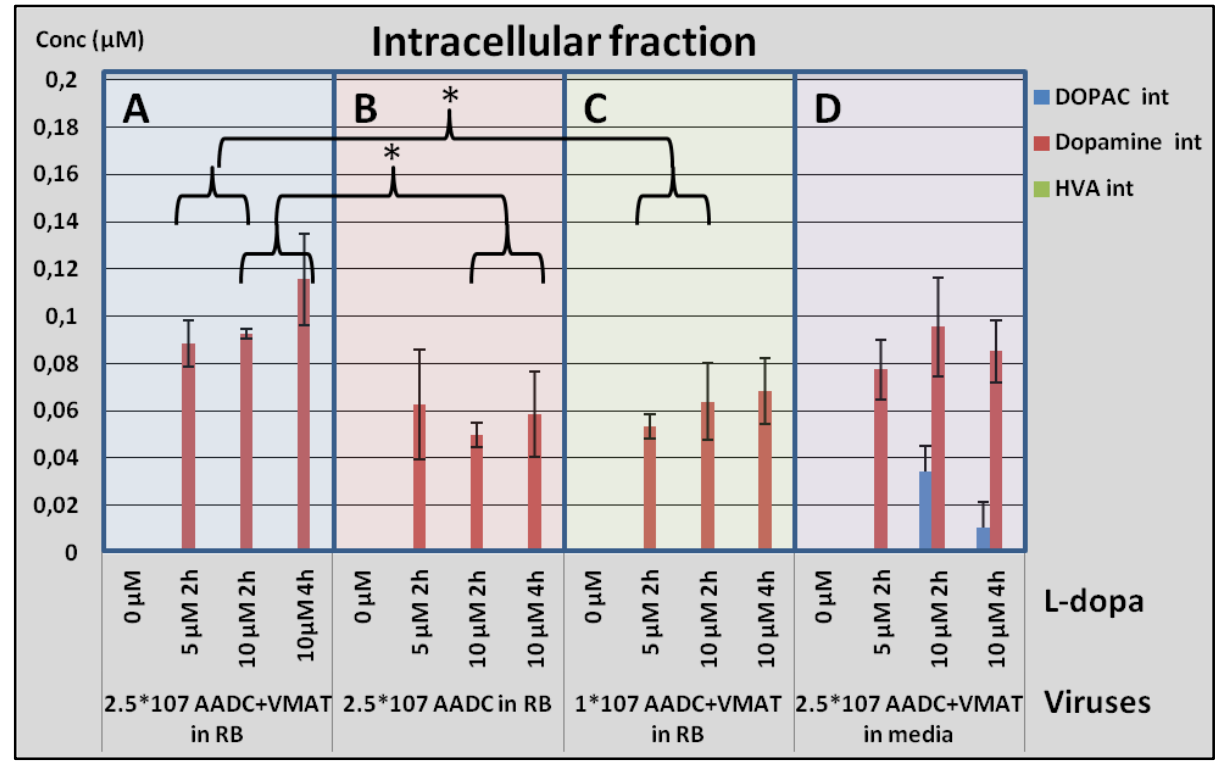

Figure 51) Dopamine, DOPAC and HVA measured in the intracellular fraction of dopamine-producing neurons measured by HPLC. $*=2$ tails t-test $<0.05$. Error bars $=$ standard deviation.

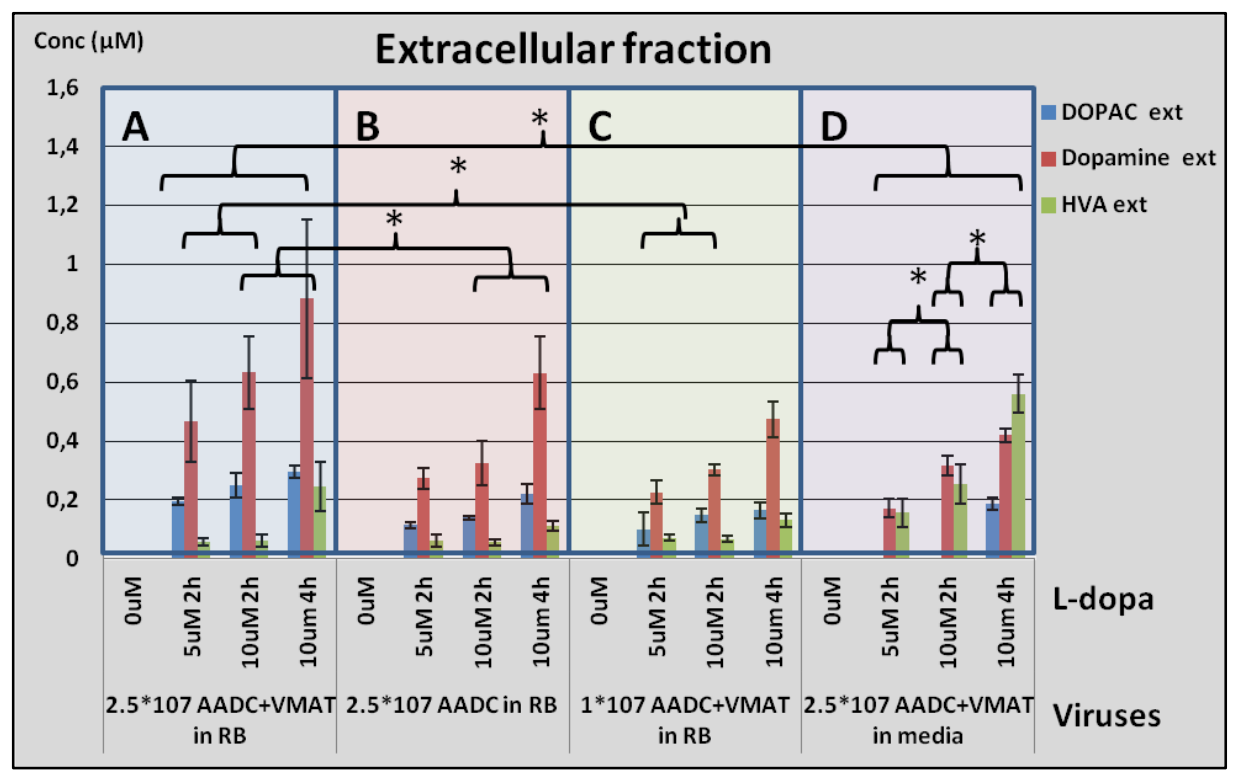

Figure 52) Dopamine, DOPAC and HVA concentration in the extracellular fraction of dopamine-producing neurons measured by HPLC. $*=2$ tails t-test $<0.05$. Error bars $=$ standard deviation.

In order to verify the repetitiveness of the dopamine production transduced neurons underwent three cycles of L-dopa induction. Cultured media was analyzed and changed before the L-dopa addition. After 4 hour incubation media was analyzed again (time-table in figure 53). As can be noted in the graph in figure 53 neurons can synthesize DA repetitively but a decrease in production is observed. Other experiments, not reported here, indicate that this decrease is likely due to a lower viability of the culture caused mainly by the media changing; cultured media indeed is normally only refreshed ( $200 \mu \mathrm{l}$ discarded and $300 \mu \mathrm{l}$ of fresh media) to avoid excess of stress to neurons while in this experiment the whole media was changed before the addition of L-dopa. 


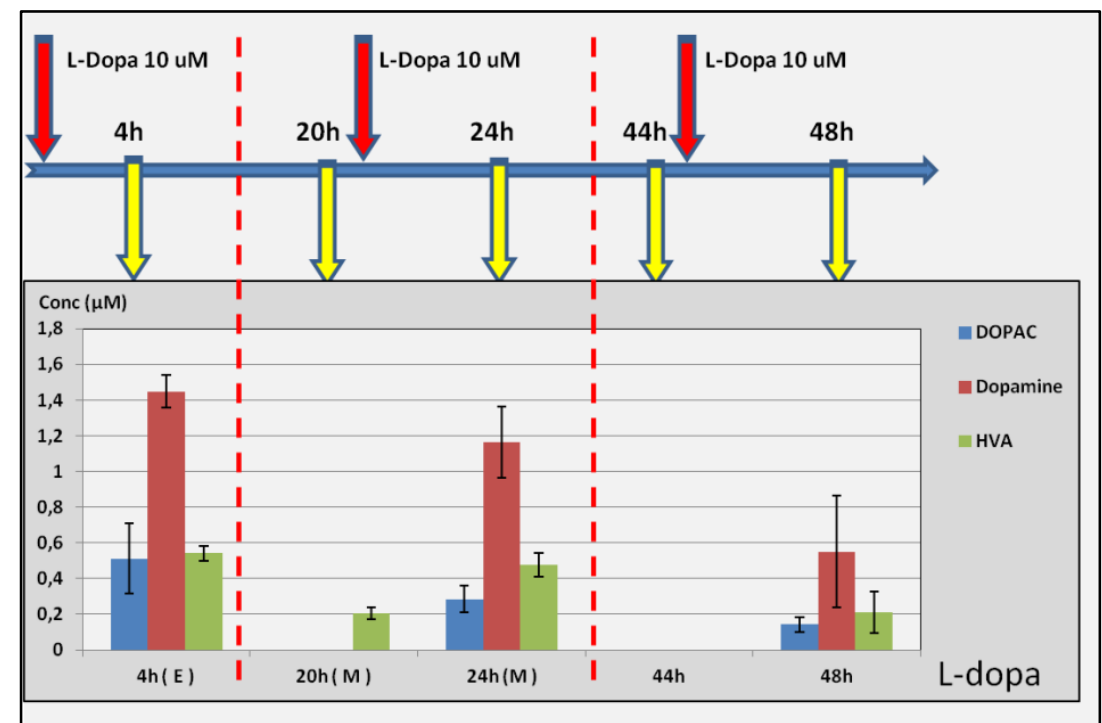

Figure 53) Reiterate dopamine production measured by HPLC in dopamine-producing neurons. $10 \mu \mathrm{M}$ of L-dopa was added, the dopamine production was measured $4 \mathrm{~h}$ later and the whole media was exchanged. After $20 \mathrm{~h}$ no dopamine was

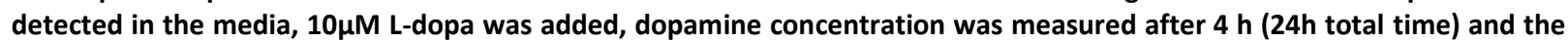
whole media was exchanged. After $20 \mathrm{~h}$ (44h total) no dopamine was detected, $10 \mu \mathrm{M}$ L-dopa was added and dopamine concentration was measured after $4 \mathrm{~h}$ ( $48 \mathrm{~h}$ total time). Error bars = standard deviation.

4.11.4 Doapmine and $\alpha$-synuclein interaction

In order to verify possible interactions between dopamine and $\alpha$-synuclein neurons were transduced with three different AAV6 vectors expressing $\alpha$-syn under the control of the synapsin promoter (AAV6 S- $\alpha$-synWB), AADC and VMAT-2. In positive controls the EGFP expressing virus AAV6 SEWB was used instead of AAV6 S- $\alpha$-synWB. Three different experiments were performed: a) the dopamine production and metabolism was assessed by HPLC, b) the viability of neurons in presence of dopamine and $\alpha$-syn was measured by BCA viability test, and c) aggregation properties of $\alpha$ synuclein were evaluated by western blot.

a) With the intent of evaluate an influence of $\alpha$-syn in DA production and release, neurons were incubated for 4 and 24 hours with $10 \mu \mathrm{M}$ L-dopa. No significant differences were observed when data were analyzed with a two tailed t-Test but, if a one tail t-Test is adopted, a significant difference can be observed in both intracellular and released fraction of dopamine after 24 hours incubation indicating a tendency of neurons to store and release less dopamine in presence of $\alpha$-syn (figure 54 ).

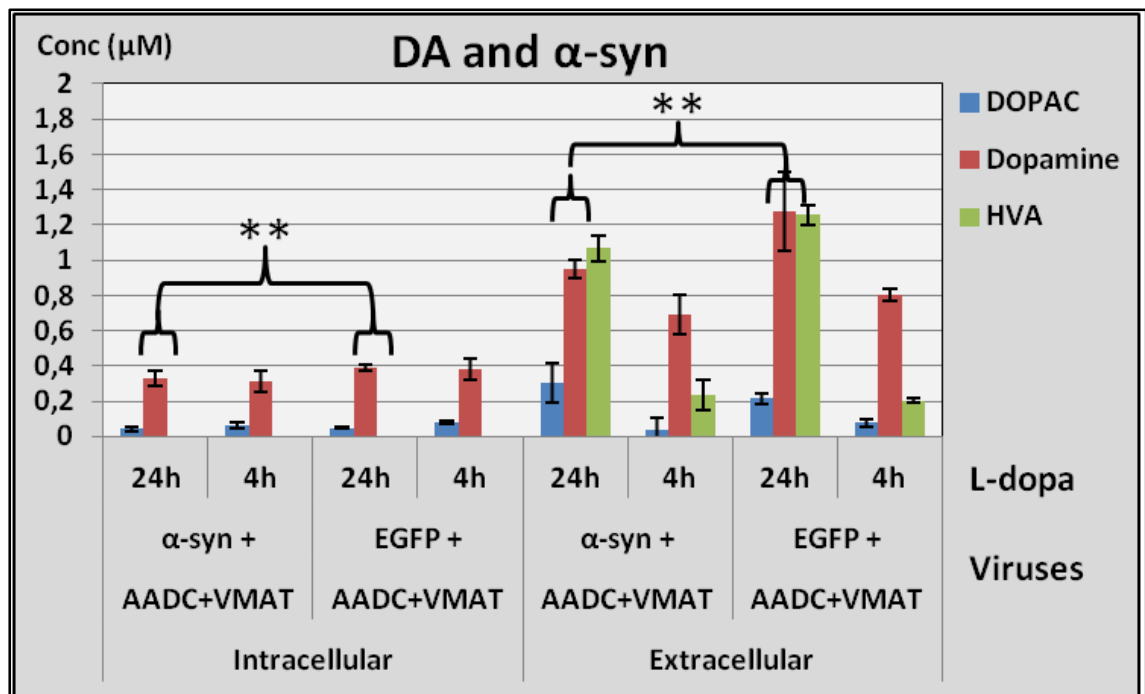

Figure 54) Dopamine, DOPAC, HVA measured by HPLC in the intra and extracellular fraction of dopamine-producing neurons co-transduced with $\alpha$-synuclein $\left(\alpha\right.$-syn) or EGFP expressing viruses. ${ }^{* *}=1$ tail t-test $<0.05$. Error bars $=$ standard deviation. 


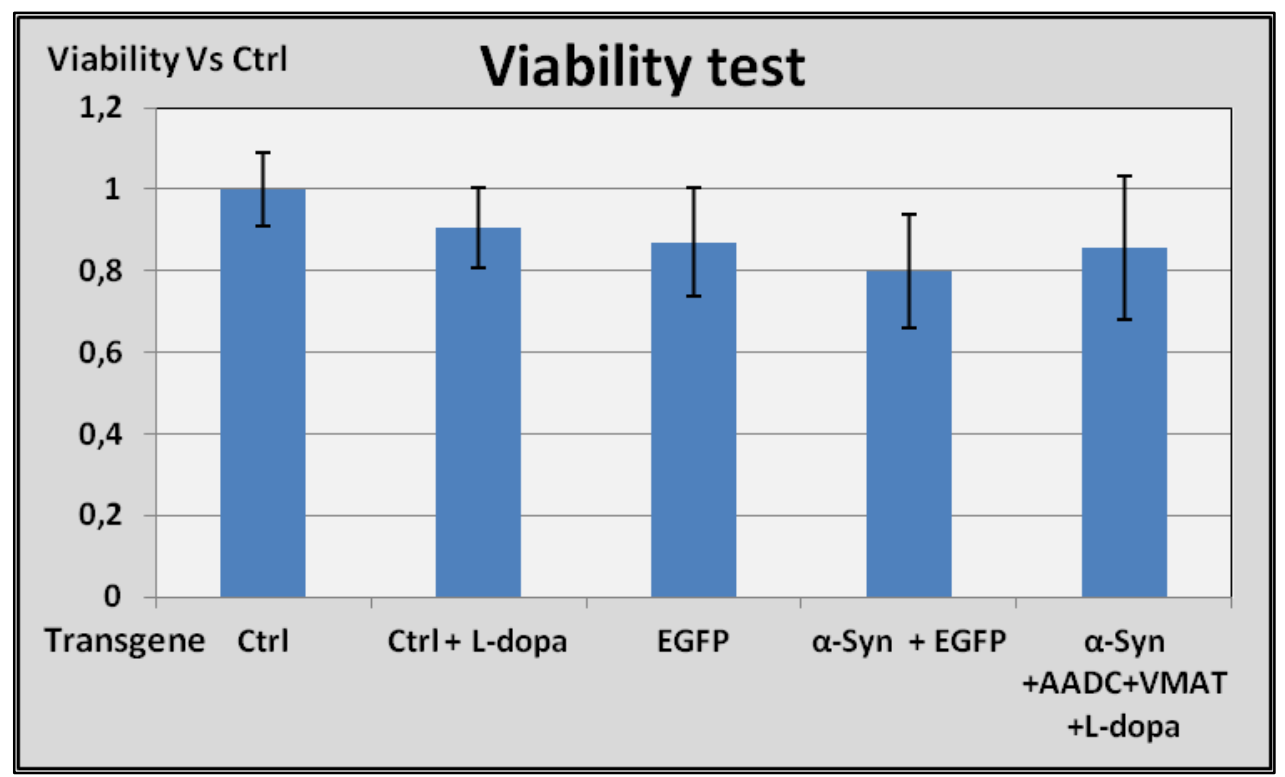

Figure 55) BCA viability test performed of primary cortical neurons transducer with viruses expressing the listed genes. $\alpha$-syn $=\alpha$-synuclein; EGFP $=$ Ctrl $=$ non-transduced control. Error bars = standard deviation.

b) To evaluate if the presence of dopamine, $\alpha$-syn, and the combination of the two has an immediate effect on neuron viability a BCA viability test was performed $48 \mathrm{~h}$ after L-dopa incubation using as a positive control both untreated cells and neurons transduced with AAV6 SEWB. No significant difference could be observed in any case (figure 55).

c) Unpublished data have shown that, in a cell-free system in vitro, the presence of dopamine trigger $\alpha$-syn aggregation (figure 56, picture a). In order to verify if this can be reproduced in primary cortical neurons, transduced cells (AADC + VMAT-2 + $\alpha$-syn) were incubated for 4,48 and 96 hours with $10 \mu \mathrm{M}$ L-dopa. Differently from the cell-free system no increase in aggregation of $\alpha$-syn was noticed both in native (figure 56 picture b) and SDS-Western blot (figure 56 picture $c$ ).

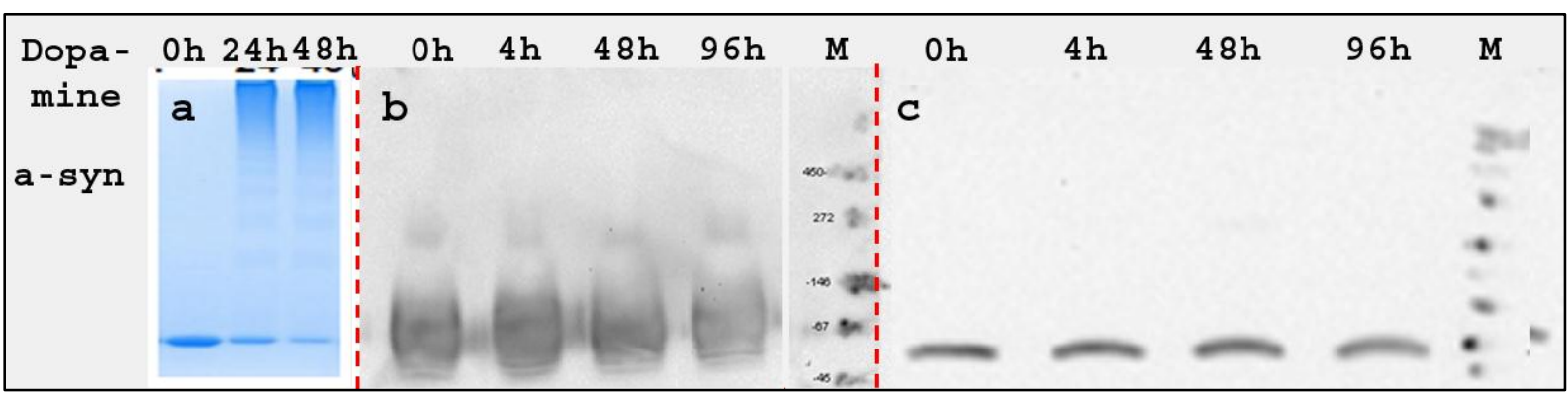

Figure 56) Western blot for $\alpha$-syn performed at different incubation times with dopamine. a) $\alpha$-synuclein was incubated in vitro in a cell-free system with dopamine; b) native western blot performed on cell lysates of dopamine producing neurons co-transduced with a $\alpha$-synuclein expressing virus; c) SDS western blot performed on cell lysates of dopamine producing neurons co-transduced with a $\alpha$-synuclein expressing virus. Time in $b$ and $c$ refer to the $L$-dopa incubation. $M=$ broad range protein marker. $\alpha$-syn aggragation can be observed in the cell-free system upon dopamine addition while no aggregation is observed in cultured neurons. a-syn $=\alpha$-synuclein. 


\section{Discussion}

\subsection{Development of a AAV vector based regulatable system for use in the CNS}

Gene therapy has been shown to be a promising tool for treating diseases currently untreatable with conventional medicine but its introduction into the clinic has been considerably delayed due to safety concerns. The application of viral-based vectors implies the insertion in the target cells of foreign DNA which will be expressed by the host cell transcription and translation systems. Different from traditional medicine, in cases of unwanted side effects, the treatment cannot be reversed. This drawback is exacerbated in the CNS where target cells consist of post-mitotic cells like neurons. One gene therapy approach for PD treatment consists of the overexpression of NFs such neurturin [38]or GDNF [37] in order to promote the DA neuron survival; the positive effects of GDNF on DA neuron survival have been demonstrated [27] but side effects due to overexpression of the NF or mistargeting of the delivery vector, such as weight loss [40], aberrant sprouting of neurons [41] and $\mathrm{TH}$ downregulation [42] have been observed in animal models. Moreover, side effect may be due to the overexpression of neurturin or viral-mis-targeting, has been observed in a phase $1 /$ II clinical trial where a neurturin AAV2 expressing virus was injected bilaterally in the putamen of PD patients [39]. As a consequence only a few clinical trials have been performed in this direction and only advanced patients that did not show any benefits from traditional treatments were involved. This might have lead to a partial failure when NFs were administrated as not enough surviving target cells were present.

In order to increase the safety profile of gene therapy, a regulated protein expression tool to control the transgene expression in case of unwanted side effects would be recommendable. In this project we explored, for the first time, the possibility to combine a mifepristone-based gene regulatable system together with the AAV delivery tool for application in the CNS.

To promote its use in clinical trials, an optimal system should have the following features [50, 51]:

$\checkmark$ Ideal "zero" basal level of expression in the non induced state, or at least, a level without biological effects

$\checkmark$ Biologically relevant expression in the induced state

$\checkmark$ Constituted by elements of human origin

$\checkmark$ Positively induced

$\checkmark$ Demonstrated safety profile of the inducer in human

$\checkmark$ Dose responsiveness

$\checkmark$ Fast kinetics

$\checkmark$ Repetitiveness of induction

$\checkmark$ No pleiotropic effects

$\checkmark$ Not affecting endogenous gene expression

$\checkmark$ be region or cell specific

One of the major problems shared by regulatable gene promoter systems is the difficulty to achieve a "zero" level of transgene expression in the off-state while maintaining a biologically relevant level of transgene expression in the on-state. In this project the effects on the transgene expression level of various elements (isolators, transcriptional control elements, promoters, cassettes configuration, serotypes of AAV) have been studied; the combinations of constructs that gave the best compromise 
between a low level of transgene (EGFP and GDNF) expression in the off-state and a high expression of the same proteins in the on-state consisted of AAV5 Gfabc1d pSwS + AAV5 3TBUTEB (figure $10 \mathrm{~g}+1$ ) or AAV5 SpSwS + AAV5 3TBUTEB (figure $10 \mathrm{~h}+1$ ). Despite the omission of the enhancing transcription element WPRE, the inclusion of 3TBs to isolate the ITR's effect, and the employment of AAV5 serotype, which delivers less viral genomes per cell comparing to AAV6, a low but detectable level of expression in the off-state was observed both when EGFP and GDNF were expressed. By measuring GDNF concentration in the striatum by ELISA, a 2 fold increase of protein concentration in the offstate has been observed in the injected hemisphere compared to the endogenous expression measured in the contra-lateral hemisphere. Upon induction of the system with MF, an 18 fold overexpression of GDNF over the endogenous level was measured. Hadaczek et al., studied a ramamycin-based regulatable system in AAV vectors for GDNF expression in rat brain and a ratio of about 50:1 in protein concentration between the off- and the on-states was observed by ELISA. Despite the high induction ratio, it has to be noted that the GDNF expression level in the off-state resulted to be 20 folds higher comparing to the endogenous level [63]. Our data appear to be similar to what was observed using the tet-on system in AAV or LV where the level of GDNF expression in the off-state was 1 to 2 fold higher compared to the endogenous level while a 7 to 12 fold overexpression of GDNF was measured in the on-state. [55-58]. Contrarily, the tet-off system appears superior both for induction capacity, displaying a 500 to 1000 fold induction between the off- and the on-states, and for basal level of expression in the off-state which could not be distinguished from the endogenous GDNF expression. Nevertheless it has to be noted that, in order to maintain such a low level of GDNF expression in the off-state, a high dosage ( $500 \mathrm{mg} / \mathrm{kg}$ diet) of doxycycline had to be provided to the animals [54]. The pSwitch system activity in the brain, mediated by a HSV viral vector, has been evaluated only in one study performed by Oligino et al. In this study Lac $Z$ was used as a reporter gene and, similar to our result with EGFP, a low but detectable level of expression in the non induced state could be measured while a much higher ratio ( 150 fold) of protein expression between the off- and the on-state was observed upon MF administration. [90]. This higher induction rate might be due to the use of only one HSV vector comparing to our 2 vectors co-transduction protocol. If only one vector is used $100 \%$ of infected cells contain the two cassettes while, if two separated vectors are used, only co-transduced cells can overexpress the GOI upon induction, thus lowering the transgene expression in the on-state. In this project several one vector systems have been tested but they resulted in a much higher transgene expression in the off-state and, as a consequence, they were not investigated further. Moreover the system used by Oligino was an older version of the pSwitch system which included the Herpes virus protein VP16 activation domain instead of the human p65 activation domain rendering the system slightly different and not fully comparable.

In this study it has been observed a 2 fold GDNF overexpression in the off-state and an 18 folds overexpression of the same protein in the on-state (levels normalized with the endogenous GDNF expression). In order to evaluate if both these levels of GDNF expression display a relevant biological effect we tested (results not presented here) the ability of the pSwitch system in the on- and if the off-states to protect the dopaminergic fibers in the striatum from the effect of the DA neuron specific toxin 6-OHDA (Adeno-associated virus mediated, mifepristone-regulated neurotrophic factor expression in the brain; article submitted). 
Four groups of animals were prepared:

a) AAV5 Gfabc1dpSwS + AAV5 3TB UT-GDNF-B induced with MF

b) AAV5 Gfabc1dpSwS + AAV5 3TB UT-GDNF-B not induced

c) AAV5 Gfabc1d GDNF WB (GDNF constitutively expressed by the Gfabc1d promoter; positive control)

d) AAV5 Gfabc1dpSwS + AAV5 3TB UTEB induced with MF (EGFP expressing system upon MF induction; negative control)

Viruses were injected in rat striatum and the MF induction was performed 5 days prior 6-OHDA injection in order to overlap the toxin-induced lesion with the GDNF expression in the pSwitch induced group. Results showed that, while the induced group displayed protection from the toxin, not-induced group exhibited a lesion comparable to the negative control group expressing EGFP instead of GDNF; the lesion was verified by apomorphine induced rotation, cylinder test, and IHC. This proof of principle experiment demonstrated that a 2 folds GDNF overexpression in the off-state do not have relevant biological effect for this paradigm while the GDNF expression in the on-state do have a biological effect. Importantly no weight loss and no TH downregulation has been observed in both groups of animals injected with the pSwitch system (induced and not induced). Similar results in regard of the absence of side effects have been reported by Manfredsson et al. when the tet-off system was used for controlling the GDNF expression [54].

While a good immunological toleration of AAV vectors as delivery tool in the CNS has been assessed in various clinical trials $[118,119]$, the beneficial effect of inclusion of a regulatable system for controllable gene expression is a topic of debate [120,121]; immunological reaction against the regulating machinery might indeed lead to a failure of the treatment. In order to address this aspect several studies on the most widely used tet-on system have been performed. The tet-on system has shown no immune reaction over a period of 5 years when expressed by using an AAV vector as a delivery tool in the retina of non-human primates [59]. One the contrary, a strong humoral and cellular mediate immune response resulting in rapid loss of tet-mediated control of transgene expression was observed after intramuscular injection in non-human primates of a adenoviral vector expressing the tet-on system[60]. Importantly, this immune response could not be prevented by engineering the bacterial elements of the tet system [122].

In this project we propose the employment of the pSwitch system that, differently from the tet-on and tet-off systems is mainly constituted by human elements (excluding the GAL4 DBD) which might lower the immunogenicity of the system. In our experiment we assessed that the observed astroglyosis was probably due to the injection needle and that microglia were not activated in response of the expression of the pSwitch protein. These results cannot be considered definitive but indicate that it is reasonable to further investigate the immunogenic properties of the system in nonhuman primates.

An important feature for promoting the use of regulatable systems in human patient is that the administration of the inducing molecule has to be safe and well tolerated. Moreover it is highly desirable that the inducing drug has a positive effect by activating the expression of the protein rather than repress it. Due to this last requirement, the tet-off system, which is negatively regulated and need continuous administration of the inducer for the silencing of the transgene expression, is not advisable for use in humans. The pSwitch system, in order to induce the gene expression, makes 
use of the already FDA (Food and Drug Administration) approved steroid mifepristone. Mifepristone is a synthetic 19-norsteroid with progesterone and glucocorticoid antagonist activity; this compound is used as a component for medical termination of pregnancy $(\sim 10 \mathrm{mg} / \mathrm{kg} ; 600 \mathrm{mg}$ tablet; body weight considered $=60 \mathrm{~kg})$, for contraception $(\sim 0,02-0,2 \mathrm{mg} / \mathrm{kg})$, for treatment of breast and ovarian cancer ( 1 mg/kg/day), for HIV treatment (Clinical trial phase I/II 1-4 mg/kg) [78], and for major depression $(10 \mathrm{mg} / \mathrm{kg})$ [79]. In general, MF can be used in any situation where a blockage of the glucorticoid activity is desired which is achieved with a dosage $>4 \mathrm{mg} / \mathrm{kg}$ of MF [123]. Importantly, MF administration had been proven to be safe over several months of treatment with only few minor adverse side effects such fatigue and rush but no disturbance of the immune parameters [124]. In the pSwitch system MF acts as an agonist binding to the hPR-LBD of the pSwitch protein and promoting its dimerization and the subsequent transcription of the GOI [69].

Similarly to the dosage used by Oligino et al. (25 mg/kg IP for two days), in this project the administrated MF dosage consisted of $20 \mathrm{mg} / \mathrm{kg} /$ day injected intraperitoneally in three consecutive days; by converting this dosage using the FDA approved normalization to body surface area from rats to human it results that $20 \mathrm{mg} / \mathrm{kg}$ in rats corresponds to $3.2 \mathrm{mg} / \mathrm{kg}$ in human, dosage well below to what has already been showed to be safe. Moreover, we have demonstrated that the EGFP expression can be induced with dosages of 10 and $5 \mathrm{mg} / \mathrm{kg}$ in rats which correspond to 1.6 and 0.8 $\mathrm{mg} / \mathrm{kg}$ in human. As MF is orally available for use in human, further experiments to evaluate minimal dosage and ideal route of administration of the drug need to be performed in rodents and nonhuman primates.

The kinetic of expression is another important aspect of an inducible gene expression system which should, ideally, display a fast response both in the induction and in the repression of the transgene expression. In this project the induction was performed over three days and the expression was evaluated the third day after the last day of induction indicating a reasonable fast activation of the system ( 5 days after the first induction). Transgene expression has been shown to decrease at basal level 4 weeks after induction in rats (GDNF tested by ELISA and EGFP tested by IHC) and 2 weeks after induction in mice (EGFP evaluated by in vivo imaging). Another study adopting the pSwitch system expressed through a systemically administrated adenovirus in mice has shown that the transgene reach the peak of expression already $12 \mathrm{~h}$ post induction and the expression returns to basal level after one week [89]. Similar results were observed with the pSwitch system administered in the mice muscles through a plasmid based method [125]. These results indicate that the pSwitch system might have a faster kinetic comparing to what we assumed ( 5 days for induction and 4 weeks in rats and 2 weeks in mice for silencing) but, as these studies were performed in different target areas, further studies are needed to confirm that this assumption is true for a pSwitch system applied in the CNS through AAVs.

The ability of the regulatable gene expression system described here to be repeatedly activated is confirmed by the three consecutive induction analyzed with the system injected in the rat striatum and the 6 cycles of activation performed with the viral system injected in mouse cortex and evaluated by in vivo imaging.

The requirement that the induction of transgene expression should not interfere with the endogenous gene expression is fulfilled by different observations. The initiation of DNA transcription is based on the binding of the GAL4 DBD to the GAL4 binding sites, sequences of yeast origin and present exclusively in the pSwitch cassettes. No evidence of an unspecific binding of the pSwitch 
protein to the mammalian DNA has been observed. Moreover the use of recombinant AAVs implies that the transduced DNA stays in an episomal form into the nucleus avoiding integration with the host genome that might lead to disturbance in the expression of other genes. As shown by the viability test in culture, the pSwitch protein might have some toxic effect only if expressed at high level but the two vectors system employed here implies a low utilization of the vector expressing the pSwitch protein meaning a decrease of a possible toxic effect. Moreover toxic effects have been observed only in cultured neurons while no toxicity of the pSwitch system has been observed with the viruses injected in rat striatum as demonstrated by lack of microglia activation or efficiency of EGFP expression when the system was repetitively induced. On the other hand, a decrease of EGFP expression upon MF induction was observed by in vivo imaging after the first induction with the pSwitch system injected in mouse cortex; this decrease might have been due to the presence of the cranial window which might have triggered an inflammation in the area of injection and a subsequent loss of transduced cells.

Cell type specificities can be achieved by adopting a cell specific promoter to express the pSwitch protein. In this study three promoters have been evaluated: the ubiquitous promoter Uptk, the astrocyte Gfabc1d promoter, and the neuron specific synapsin promoter. While we could demonstrate the specificity of the synapsin promoter more studies are needed to assess the tropism of the astrocytic promoter which was shown to be $100 \%$ astrocytic specific in transgenic mice [101] but that showed consistent activity in non astrocytic cell types in this project; this unspecificity might be due to the formation of concatamers upon transductions with the ITRs overcoming the specificity of the promoter.

In conclusion it was shown that the pSwitch regulatable gene expression system in AAV described in this project fulfill most of the criteria required for the use in humans: it shows really low expression of the transgene in the off-state (comparable to the tet-on system) and a biologically relevant effect in the on-state (if GDNF is expressed and the 6-OHDA paradigm is considered), it is positively induced with a drug already approved for use in human, it displays dose-dependence, fast kinetic, and repetitiveness of induction, and no side effects due to pleiotropic activity has been observed. Further studies are nevertheless needed to confirm the lack of an immune response, to define the best route of mifepristone administration, and for a more cell-specific expression which can be obtained by further manipulations of the promoters. Moreover, more studies need to be performed in order to eliminate the background expression by engineering the UAS-TATA promoter.

\subsection{GDNF expression in the 6-OHDA rat model}

The ability of GDNF to protect [34] or restore [126] a lesion of the dopaminergic system in the striatum due to a 6-OHDA application have been demonstrated. Therefore, after having characterized the pSwitch system using EGFP as expressed gene, our goal was to assess if a short pulse of expression from a regulatable vector expressing GDNF instead of EGFP might have any restorative effect on the same 6-OHDA rat model of PD; moreover it was important to verify if the low but detectable expression in the off-state might have a biological effect on animals. With this purpose, animals were first injected with 6-OHDA to induce a DA neuron loss and once the lesion was confirmed by the three motor tests, Apomorphine induced rotation, cylinder test and corridor test, they were divided in 4 groups: 
a) Treated group with the pSwitch system induced with MF

b) Not treated group with the pSwitch system not induced

c) Positive control expressing GDNF constitutively

d) Negative control expressing EGFP

The first part of the experiment, consisting in inducing the DA neuron lesion in the left striatum, gave the expected results confirmed by all tests: a contralateral rotation of about 250 turns/hour upon apomorphine injection, a preferential left paw use in the cylinder test, a preferential left food withdrawn in the corridor test and a depletion in the total dopamine amount of about $50 \%$ comparing to the unlesioned contra-lateral striatum. Functionality of the viruses was confirmed by GDNF ELISA on animals sacrificed immediately after the first MF induction in the treated group. Results of this test show that, a high level of expression was given by the GDNF constitutiveexpressing virus in the positive control (> $2000 \mathrm{pg} / \mathrm{mg}$ tissue), no increase in the NF level was observed in the EGFP negative control ( $3-4 \mathrm{pg} / \mathrm{mg}$ tissue), a two folds increase in GDNF level comparing to the contra-lateral side was measured in animals injected with the pSwitch system but not induced with MF ( $\sim \mathrm{pg} / \mathrm{mg}$ tissue), and a 18 fold overexpression versus the endogenous level when animals injected with the pSwitch system were induced with $20 \mathrm{mg} / \mathrm{kg}$ of $\mathrm{MF}(\sim 54 \mathrm{pg} / \mathrm{mg}$ tissue).

The second part of the experiment, which aimed to restore the confirmed lesion by a short MFinduced GDNF expression, leaded to discordant results. In all three motor behavior tests a comparable amelioration was observed but each group presented high standard deviations indicating great variability within the groups. Moreover, in several cases, the motor behavior tests displayed variability in the same animal when tested at the different time points. Dopamine evaluation at the end of the experiment showed that total dopamine level in the striatum was not increased in any of the groups. ELISA for GDNF gave the expected results with a high level of GDNF expression in the positive control, and the basal two-fold overexpression level comparing with the endogenous expression in both groups injected with the pSwitch system; in this case, the pSwitch group induced with MF, was sacrificed four weeks after the last induction and therefore this low level of expression was expected. A TH staining to mark dopaminergic fibers in the striatum was performed but a clear explanation could not be defined. Indeed, in some cases, brains belonging to different rats displayed a similar size of the lesion but the animals were performing completely differently in the motor tests.

Different causes might have leaded to these results: it has been demonstrated that GDNF concentration in cell culture should lie within a specific range otherwise, if a certain threshold is reached, the presence of the protein becomes toxic for cells [127]. In our positive control we measured a concentration of more than $2000 \mathrm{pg} / \mathrm{mg}$ tissue, meaning 500-1000 times higher than the normal endogenous level. This high concentration might have lead to invalid results in the positive control.

Another explanation for the variability measured in this experiment can be that the size of the lesion was "border line" meaning that a spontaneous recovery was possibly masking the effect of the treatment. A comparable outcome have been already noticed in a similar experiments in which, after a 6-OHDA lesion, rats were injected with AAV vectors expressing GDNF under the control of the regulatable system Tet-on [57]. 
In conclusion more analysis should be accomplished in which the behavioral data, the biochemical data and the results of the dopaminergic neuron staining (TH), are compared at the level of a single animal in order to define a possible pattern in the results or to identify specific loci of the lesion associated with specific motor behavior results.

\subsection{Dopamine producing neurons}

In PD several areas of the brain display progressive neuronal loss and formation of Lewy bodies and, out of them, the loss of DA neurons in the substantia nigra seems to be responsible for the motor deficits. Therefore it would be of great interest to study the effect of $\alpha$-synuclein, or the role of other protein involved in PD (LRRK2, DJ-1, Parkin, PINK-1, ATP13A2), directly in this particular population of neurons. Moreover, un-published data showed that, in a free-cell system in vitro, the presence of dopamine can trigger the $\alpha$-synuclein aggregation hypothesizing a role of the catecholamine in Lewy bodies formation. As DA neurons account only for a small fraction of the total number of neurons in the rat brain, it is not possible to obtain primary culture with more than $10 \%$ of DA neurons [110]. LHUMES (Lund human mesencephalic) cells [128] have been used to study the release of dopamine but, being an immortalized cell line, they differ substantially from DA neurons. In alternative, dopaminergic-like neurons can be obtained from the emerging field of stem cells research by starting from different source of pluripotent cells which can be cultured and provided with the appropriate stimuli $[127,129]$.

In this project we aimed to obtain dopamine-producing neurons by transducing primary cortical neurons with AAVs expressing the essential genes responsible for the catecholamine production. These genes are TH, to convert L-tyrosine into L-dopa, GCH1, for the synthesis of the TH co-factor BH4, AADC, to convert L-dopa into dopamine, and VMAT-2 which sequestrate dopamine into vesicles. Similar approaches have been already followed by transducing fibroblast with AADC + VMAT-2 and promoting dopamine production by incubating cells with L-dopa [130] or by transducing primary cortical neurons with a tri-cistronic lentiviral vector expressing TH, AADC and GCH-1 and promoting dopamine production by incubating cells with the precursor L-tyrosine.

A high and reproducible dopamine synthesis was obtained by transducing neurons with AADC or with $A A D C+V M A T$ and promoting the dopamine production by incubating cells with the precursor Ldopa.

Our experiments agree with those previously published [130] and highlight the importance of the presence of VMAT-2 in increasing both the intra and the extracellular fraction of newly synthesized dopamine. Studies on the amount of the inducer L-dopa, time of incubation, and incubation buffers displayed a good reproducibility of the system regarding both the intra and the extracellular fraction of the synthesized dopamine and its metabolites.

Considering the possible interactions between dopamine and $\alpha$-synuclein, we showed a lack of toxic effects due to dopamine, $\alpha$-syn, or the combination of the two, in the neuronal culture at least for the time-frame considered in this experiment (48h). The $\alpha$-synuclein aggregation tendency in presence of dopamine, observed in a free-cell system in vitro, was not confirmed when western blots were performed on cultured neurons indicating the ability of cells to prevent this aggregation at least for the time considered (96h). Considering the dopamine production, a one tail t-Test showed a tendency of $\alpha$-synuclein to affect negatively the catecholamine synthesis and release; more 
experiments including extended time points and different amount of synthesized dopamine are needed to confirm this hypothesis.

In conclusion we developed an easy, regulatable and reproducible system for dopamine synthesis in primary cortical neurons that can be used for study the interaction of the neurotransmitter, and its oxidative role, with other components of PD.

\subsection{Considerations about AAV transduction in the CNS}

In this project two different serotypes have been used, AAV5 and AAV6 which show different transduction properties due to their differences in the capsid which is, as a consequence, differently recognized by cells. AAV6 was chosen at the beginning for the ease of production and the ability in transducing primary cortical neurons in culture while AAV5 serotype was used in a later stage in order to obtain a lower release of genome copies per cell resulting in a decrease in EGFP expression level in transduced cells.

A drawback of AAV viruses is the limited DNA packaging capacity ( $\sim .8 \mathrm{~kb})$ and, as a consequence, cotransduction with 2 vectors expressing different proteins has been routinely performed without any observed decrease in the transduction ability. This feature was observed with different serotypes [114-116] including serotype $5[114,117]$.

During our experiments we observed that this assumption might have to be reconsidered at least when a triple co-transduction with AAV5 is performed. In order to facilitate the identification of the transduced area in the experiment aiming to evaluate consecutive cycles of induction, a third AAV5 virus, expressing DsRed under the control of the neuronal promoter synapsin (AAV5 SDsRedWB figure 10-c), was co-injected together with the two AAV5 vectors forming the pSwitch system (AAV5 Gfabc1d pSwS + AAV5 3TB-UTEB figure $10 \mathrm{~g}+1)$. The outcome of this experiment was a drastic reduction of the EGFP expression in the on-state both considering the number of cells and the EGFP expressed within single cells. This observation was confirmed by repeating the double and triple cotransduction in a new group of animals and using viruses from the same production batch. An overload of the virus cell receptors can be excluded as the sum of the titres of the three vector $\left(0.3 * 10^{8}\right.$ TU AAV5 Gfabc1d pSwS $+1 * 10^{8}$ TU AAV5 3TB-UTEB $+0.5^{*} 10^{8}$ TU AAV5 SDsRedWB $=1.8^{*} 10^{8}$ TU total amount) is considerably lower compared to titres that have been used in other experiments (for example $2.75 * 10^{8} \mathrm{TU}$ when the one vector system was used). We cannot propose a conclusive explanation of this phenomenon but, considering that after transduction the AAVs genomes stay in an episomal structure forming concatamers into the nucleus, we hypothesize that some rearrangements in the DNA sequences might have occurred. Moreover, the DsRed protein in the third vector is under the control of the synapsin promoter which is known to promote a high level of expression; this high activity of the synapsin promoter might give rise to a competition for the transcription elements necessary for DNA transcription leading to a poor activity of the UAS TATA promoter present in the pSwitch system.

Adeno associated viruses make use of surface cell receptors to mediate cell-entry and the set of needed receptors is topic of debate. Studies performed by Seiler et al. in airway epithelial cells demonstrated that AAV5 and AAV6 use a completely different set of receptors to mediate the cellentry indicating no competition of the two serotypes in cell transduction [113]. Contrarily, Asokan et al. states that both AAV5 and AAV6 are using the $\alpha 2,3 \mathrm{~N}$-linked sialic acid receptor to mediate their entry into the cell [131]. During the course of this project AAV5 expressing EGFP and AAV6 
expressing DsRed have been used together to co-transduce mouse cortex; IHC on these brains revealed a repetitive pattern in which the DsRed (AAV6) was expressed in the proximity of the point of injection while the EGFP (AAV5) was expressed exclusively in the periphery. These results indicate a high competition for the receptor in favor of the AAV6 serotype which validates Asokan's assumption in regarding, at least, the co-transduction in mouse cortex.

The inefficiency of AAV5 triple transduction and the strong competiveness between AAV5 and AAV6 for cell entry demonstrate that many aspects of AAV biology have to be still elucidates for a proper use of this delivery tool both in the CNS and in other tissues.

\subsection{Outlook}

We have demonstrated to have developed a tight and controllable gene expression system that can be considered as an alternative of the most widely used tet-on system for possible use in clinical trials. As stated already above, forthcoming studies should aim to:

- optimize oral mifepristone administration

- test the immunological profile of the pSwitch system in non-human primates

- optimize promoters for cell tropism and lowering the background expression 


\section{Summary}

Parkinson's disease (PD), the second most common neurodegenerative disease in the western society, affects more than $1 \%$ of the population and its impact on the society increases with the increasing average age. Current treatments for Parkinson disease, based on L-dopa administration, are only symptomatic and the neural loss is not prevented. As a consequence treatments become less effective and lead to the onset of side effects such dyskinesia. New treatments are, therefore, highly demanded. New hopes come from the emerging field of gene therapy but its employment for clinical trials has been considerably slowed down due to safety concern regarding its intrinsic irreversibility. In order to circumvent this drawback the availability of a regulatable gene expression system would be favorable. The most widely used gene regulatable system is based on the tetoperon. Despite the good characteristics in gene regulation its employment for treatment in humans is unlikely due to the immunological reactions observed when applied in non-human primate muscle.

In this project we propose, for the first time, the employment of the mifepristone-based pSwitch system for regulated gene expression in the CNS by adeno-associated viral (AAV) vectors. The pSwitch system has the advantage to be mainly constituted of human protein components, which should decrease the possibility of an immune reaction against the elements of the system. Moreover, the inducing drug mifepristone is already approved for use in humans.

In the first part of the study, using EGFP as reporter gene, the effect of different transcription elements and virus serotypes is evaluated. The aim was to obtain a good compromise between an ideal "zero" level of expression in the off-state and a high level of expression in the on-state. Results obtained here are compared with alternative gene regulatable systems including the tet-system.

Once defined the combination of vectors, EGFP was substituted with the neurotrophic factor GDNF and the biological effect of this protein was evaluated in the on- and in the off-states. Using the dopaminergic neuron selective drug 6-OHDA, a rat model of PD was generated. Viruses encoding the pSwitch system for inducible GDNF expression were injected in the area of the lesion and the effect of a short expression of the protein was evaluated. Results of this experiment are controversial and reasons for this are discussed.

In a second project we aimed to generate dopamine-expressing neurons by AAV transduction. For this purpose primary cortical neurons in culture were infected with AAVs expressing the enzymes necessary for the synthesis of the catecholamine. Dopamine-producing neurons served to evaluate possible interaction between dopamine and $\alpha$-synuclein, the major component of Lewy bodies in PD. No increase in aggregation of $\alpha$-synuclein was observed but a tendency in decreasing dopamine production and release was measured in presence of $\alpha$-synuclein. 


\section{References}

1. Bredesen, D.E., R.V. Rao, and P. Mehlen, Cell death in the nervous system. Nature, 2006. 443(7113): p. 796-802.

2. Rubinsztein, D.C., The roles of intracellular protein-degradation pathways in neurodegeneration. Nature, 2006. 443(7113): p. 780-786.

3. Parkinson, J., An essay on the shaking palsy. 1817. J Neuropsychiatry Clin Neurosci, 2002. 14(2): p. 223-36; discussion 222.

4. Fahn, S., The history of dopamine and levodopa in the treatment of Parkinson's disease. Movement Disorders, 2008. 23: p. S497-S508.

5. Jankovic, J., Parkinson's disease: clinical features and diagnosis. J Neurol Neurosurg Psychiatry, 2008. 79(4): p. 368-76.

6. Rao, G., et al., Does this patient have Parkinson disease? JAMA, 2003. 289(3): p. 347-53.

7. Lesage, $\mathrm{S}$. and A. Brice, Parkinson's disease: from monogenic forms to genetic susceptibility factors. Human Molecular Genetics, 2009. 18(R1): p. R48-59.

8. Polymeropoulos, M.H., et al., Mutation in the alpha-synuclein gene identified in families with Parkinson's disease. Science, 1997. 276(5321): p. 2045-7.

9. Nuytemans, K., et al., Genetic etiology of Parkinson disease associated with mutations in the SNCA, PARK2, PINK1, PARK7, and LRRK2 genes: a mutation update. Hum Mutat, 2010. 31(7): p. 763-80.

10. Eriksen, J.L., Z. Wszolek, and L. Petrucelli, Molecular pathogenesis of Parkinson disease. Arch Neurol, 2005. 62(3): p. 353-7.

11. Youle, R.J. and D.P. Narendra, Mechanisms of mitophagy. Nat Rev Mol Cell Biol, 2011. 12(1): p. 9-14.

12. Pankratz, N., et al., Mutations in DJ-1 are rare in familial Parkinson disease. Neurosci Lett, 2006. 408(3): p. 209-13.

13. Vilarino-Guell, C., et al., ATP13A2 variability in Parkinson disease. Hum Mutat, 2009. 30(3): p. 406-10.

14. Davis, G.C., et al., Chronic Parkinsonism secondary to intravenous injection of meperidine analogues. Psychiatry Res, 1979. 1(3): p. 249-54.

15. Obeso, J.A., et al., Missing pieces in the Parkinson's disease puzzle. Nat Med, 2010. 16(6): p. 653-61.

16. Braak, H., et al., Staging of the intracerebral inclusion body pathology associated with idiopathic Parkinson's disease (preclinical and clinical stages). J Neurol, 2002. 249 Suppl 3: p. III/1-5.

17. Kirik, D., C. Rosenblad, and A. Bjorklund, Characterization of behavioral and neurodegenerative changes following partial lesions of the nigrostriatal dopamine system induced by intrastriatal 6-hydroxydopamine in the rat. Experimental Neurology, 1998. 152(2): p. 259-77.

18. Spillantini, M.G., et al., Alpha-synuclein in Lewy bodies. Nature, 1997. 388(6645): p. 839-40.

19. Au, W.L. and D.B. Calne, A reassessment of the Lewy body. Acta Neurol Taiwan, 2005. 14(2): p. 40-7.

20. Cotzias, G.C., P.S. Papavasiliou, and R. Gellene, Modification of Parkinsonism--chronic treatment with L-dopa. N Engl J Med, 1969. 280(7): p. 337-45.

21. Rodnitzky, R.L., Upcoming treatments in Parkinson's disease, including gene therapy. Parkinsonism Relat Disord, 2012. 18 Suppl 1: p. S37-40.

22. Lozano, A.M., Deep brain stimulation for Parkinson disease. J Neurosurg, 2010. 112(3): p. 477; discussion 477-8.

23. de Lau, L.M. and M.M. Breteler, Epidemiology of Parkinson's disease. Lancet Neurol, 2006. 5(6): p. 525-35.

24. Balak, N. and I. Elmaci, Costs of disorders of the brain in Europe. Eur J Neurol, 2007. 14(2): p. e9. 
25. Kaplitt, M.G., Parkinson disease: Another player in gene therapy for Parkinson disease. Nat Rev Neurol, 2010. 6(1): p. 7-8.

26. Weltman, J.K., The 1986 Nobel Prize for Physiology or Medicine awarded for discovery of growth factors: Rita Levi-Montalcini, M.D., and Stanley Cohen, Ph.D. N Engl Reg Allergy Proc, 1987. 8(1): p. 47-8.

27. Pascual, A., et al., GDNF and protection of adult central catecholaminergic neurons. J Mol Endocrinol, 2011. 46(3): p. R83-92.

28. Ibanez, C.F., Catecholaminergic neuron survival: getting hooked on GDNF. Nature Neuroscience, 2008. 11(7): p. 735-6.

29. Kastin, A.J., V. Akerstrom, and W. Pan, Glial cell line-derived neurotrophic factor does not enter normal mouse brain. Neurosci Lett, 2003. 340(3): p. 239-41.

30. Kirik, D., et al., Delayed infusion of GDNF promotes recovery of motor function in the partial lesion model of Parkinson's disease. Eur J Neurosci, 2001. 13(8): p. 1589-99.

31. Grondin, R., et al., Chronic, controlled GDNF infusion promotes structural and functional recovery in advanced parkinsonian monkeys. Brain, 2002. 125(Pt 10): p. 2191-201.

32. Palfi, S., et al., Lentivirally delivered glial cell line-derived neurotrophic factor increases the number of striatal dopaminergic neurons in primate models of nigrostriatal degeneration. $J$ Neurosci, 2002. 22(12): p. 4942-54.

33. Kojima, H., et al., Adenovirus-mediated transduction with human glial cell line-derived neurotrophic factor gene prevents 1-methyl-4-phenyl-1,2,3,6-tetrahydropyridine-induced dopamine depletion in striatum of mouse brain. Biochem Biophys Res Commun, 1997. 238(2): p. 569-73.

34. Kirik, D., et al., Long-term rAAV-mediated gene transfer of GDNF in the rat Parkinson's model: intrastriatal but not intranigral transduction promotes functional regeneration in the lesioned nigrostriatal system. J Neurosci, 2000. 20(12): p. 4686-700.

35. Gill, S.S., et al., Direct brain infusion of glial cell line-derived neurotrophic factor in Parkinson disease. Nat Med, 2003. 9(5): p. 589-95.

36. Lang, A.E., et al., Randomized controlled trial of intraputamenal glial cell line-derived neurotrophic factor infusion in Parkinson disease. Ann Neurol, 2006. 59(3): p. 459-66.

37. Richardson, R.M., et al., Interventional MRI-guided putaminal delivery of AAV2-GDNF for a planned clinical trial in Parkinson's disease. Mol Ther, 2011. 19(6): p. 1048-57.

38. Marks, W.J., et al., Safety and tolerability of intraputaminal delivery of CERE-120 (adenoassociated virus serotype 2-neurturin) to patients with idiopathic Parkinson's disease: an open-label, phase I trial. Lancet Neurology, 2008. 7(5): p. 400-408.

39. Marks, W.J., Jr., et al., Gene delivery of AAV2-neurturin for Parkinson's disease: a doubleblind, randomised, controlled trial. Lancet Neurology, 2010. 9(12): p. 1164-72.

40. Manfredsson, F.P., et al., Nigrostriatal rAAV-mediated GDNF overexpression induces robust weight loss in a rat model of age-related obesity. Mol Ther, 2009. 17(6): p. 980-91.

41. Patel, N.K. and S.S. Gill, GDNF delivery for Parkinson's disease. Acta Neurochir Suppl, 2007. 97(Pt 2): p. 135-54.

42. Sajadi, A., et al., Long-term glial cell line-derived neurotrophic factor overexpression in the intact nigrostriatal system in rats leads to a decrease of dopamine and increase of tetrahydrobiopterin production. J Neurochem, 2005. 93(6): p. 1482-6.

43. Goff, S.P. and P. Berg, Construction of hybrid viruses containing SV4O and lambda phage DNA segments and their propagation in cultured monkey cells. Cell, 1976. 9(4 PT 2): p. 695-705.

44. Blaese, R.M., et al., T lymphocyte-directed gene therapy for ADA-SCID: initial trial results after 4 years. Science, 1995. 270(5235): p. 475-80.

45. Sheridan, C., Gene therapy finds its niche. Nat Biotechnol, 2011. 29(2): p. 121-8.

46. Lentz, T.B., S.J. Gray, and R.J. Samulski, Viral vectors for gene delivery to the central nervous system. Neurobiol Dis, 2012. 48(2): p. 179-88. 
47. Samulski, R.J., et al., Cloning of adeno-associated virus into $p B R 322$ : rescue of intact virus from the recombinant plasmid in human cells. Proc Natl Acad Sci U S A, 1982. 79(6): p. 207781.

48. Wu, Z., A. Asokan, and R.J. Samulski, Adeno-associated virus serotypes: vector toolkit for human gene therapy. Mol Ther, 2006. 14(3): p. 316-27.

49. Halbert, C.L., et al., Prevalence of neutralizing antibodies against adeno-associated virus (AAV) types 2, 5, and 6 in cystic fibrosis and normal populations: Implications for gene therapy using AAV vectors. Hum Gene Ther, 2006. 17(4): p. 440-7.

50. Manfredsson, F.P., D.C. Bloom, and R.J. Mandel, Regulated protein expression for in vivo gene therapy for neurological disorders: progress, strategies, and issues. Neurobiol Dis, 2012. 48(2): p. 212-21.

51. Naidoo, J. and D. Young, Gene regulation systems for gene therapy applications in the central nervous system. Neurol Res Int, 2012. 2012: p. 595410.

52. Zhou, X., et al., Optimization of the Tet-On system for regulated gene expression through viral evolution. Gene Ther, 2006. 13(19): p. 1382-90.

53. Loew, R., et al., Improved Tet-responsive promoters with minimized background expression. BMC Biotechnol, 2010. 10: p. 81.

54. Manfredsson, F.P., et al., Tight Long-term dynamic doxycycline responsive nigrostriatal GDNF using a single rAAV vector. Mol Ther, 2009. 17(11): p. 1857-67.

55. Chtarto, A., et al., Controlled delivery of glial cell line-derived neurotrophic factor by a single tetracycline-inducible AAV vector. Experimental Neurology, 2007. 204(1): p. 387-99.

56. Georgievska, B., et al., Regulated delivery of glial cell line-derived neurotrophic factor into rat striatum, using a tetracycline-dependent lentiviral vector. Hum Gene Ther, 2004. 15(10): p. 934-44.

57. Yang, X., et al., Reversible neurochemical changes mediated by delayed intrastriatal glial cell line-derived neurotrophic factor gene delivery in a partial Parkinson's disease rat model. J Gene Med, 2009. 11(10): p. 899-912.

58. Liu, Y., et al., Protection against aminoglycoside-induced ototoxicity by regulated AAV vectormediated GDNF gene transfer into the cochlea. Mol Ther, 2008. 16(3): p. 474-80.

59. Stieger, K., et al., Oral administration of doxycycline allows tight control of transgene expression: a key step towards gene therapy of retinal diseases. Gene Ther, 2007. 14(23): p. 1668-73.

60. Latta-Mahieu, M., et al., Gene transfer of a chimeric trans-activator is immunogenic and results in short-lived transgene expression. Hum Gene Ther, 2002. 13(13): p. 1611-20.

61. Stieger, K., et al., In vivo gene regulation using tetracycline-regulatable systems. Adv Drug Deliv Rev, 2009. 61(7-8): p. 527-41.

62. Magari, S.R., et al., Pharmacologic control of a humanized gene therapy system implanted into nude mice. J Clin Invest, 1997. 100(11): p. 2865-72.

63. Hadaczek, P., et al., Evaluation of an AAV2-based rapamycin-regulated glial cell line-derived neurotrophic factor (GDNF) expression vector system. PLoS One, 2011. 6(11): p. e27728.

64. Christopherson, K.S., et al., Ecdysteroid-dependent regulation of genes in mammalian cells by a Drosophila ecdysone receptor and chimeric transactivators. Proc Natl Acad Sci U S A, 1992. 89(14): p. 6314-8.

65. Lafont, R. and L. Dinan, Practical uses for ecdysteroids in mammals including humans: an update. J Insect Sci, 2003. 3: p. 7.

66. Shen, F., et al., Adeno-associated viral vector-mediated hypoxia-regulated VEGF gene transfer promotes angiogenesis following focal cerebral ischemia in mice. Gene Ther, 2008. 15(1): p. 30-9.

67. Liu, Y., et al., A genomic screen for activators of the antioxidant response element. Proc Natl Acad Sci U S A, 2007. 104(12): p. 5205-10.

68. Banaszynski, L.A., et al., A rapid, reversible, and tunable method to regulate protein function in living cells using synthetic small molecules. Cell, 2006. 126(5): p. 995-1004. 
69. Wang, Y., et al., A regulatory system for use in gene transfer. Proc Natl Acad Sci U S A, 1994. 91(17): p. 8180-4.

70. Keegan, L., G. Gill, and M. Ptashne, Separation of DNA binding from the transcriptionactivating function of a eukaryotic regulatory protein. Science, 1986. 231(4739): p. 699-704.

71. Carey, M., et al., An Amino-Terminal Fragment of Gal4 Binds DNA as a Dimer. Journal of Molecular Biology, 1989. 209(3): p. 423-432.

72. Nelson, M. and P. Silver, Context Affects Nuclear-Protein Localization in SaccharomycesCerevisiae. Molecular and Cellular Biology, 1989. 9(2): p. 384-389.

73. Vegeto, E., et al., The mechanism of RU486 antagonism is dependent on the conformation of the carboxy-terminal tail of the human progesterone receptor. Cell, 1992. 69(4): p. 703-13.

74. Schmitz, M.L. and P.A. Baeuerle, The P65 Subunit Is Responsible for the Strong Transcription Activating Potential of Nf-Kappa-B. Embo Journal, 1991. 10(12): p. 3805-3817.

75. Baeuerle, P.A., The inducible transcription activator NF-kappa B: regulation by distinct protein subunits. Biochim Biophys Acta, 1991. 1072(1): p. 63-80.

76. Lillie, J.W. and M.R. Green, Transcription activation by the adenovirus E1a protein. Nature, 1989. 338(6210): p. 39-44.

77. Baulieu, E.E., Contragestion and other clinical applications of $R U 486$, an antiprogesterone at the receptor. Science, 1989. 245(4924): p. 1351-7.

78. Para, M.F., et al., Phase I/II trial of the anti-HIV activity of mifepristone in HIV-infected subjects ACTG 5200. J Acquir Immune Defic Syndr, 2010. 53(4): p. 491-5.

79. Flores, B.H., et al., Clinical and biological effects of mifepristone treatment for psychotic depression. Neuropsychopharmacology, 2006. 31(3): p. 628-36.

80. Heikinheimo, O., et al., Hepatic metabolism and distribution of mifepristone and its metabolites in rats. Hum Reprod, 1994. 9 Suppl 1: p. 40-6.

81. Wang, Y., et al., Ligand-inducible and liver-specific target gene expression in transgenic mice. Nat Biotechnol, 1997. 15(3): p. 239-43.

82. Kellendonk, C., et al., Inducible site-specific recombination in the brain. J Mol Biol, 1999. 285(1): p. 175-82.

83. Kitayama, K., et al., Purkinje cell-specific and inducible gene recombination system generated from C57BL/6 mouse ES cells. Biochem Biophys Res Commun, 2001. 281(5): p. 1134-40.

84. Berton, T.R., et al., Characterization of an inducible, epidermal-specific knockout system: differential expression of lacZ in different Cre reporter mouse strains. Genesis, 2000. 26(2): p. 160-1.

85. Roman, G., et al., $P[S$ witch], a system for spatial and temporal control of gene expression in Drosophila melanogaster. Proc Natl Acad Sci U S A, 2001. 98(22): p. 12602-7.

86. Yu, Y.A. and A.A. Szalay, A Renilla luciferase-Aequorea GFP (ruc-gfp) fusion gene construct permits real-time detection of promoter activation by exogenously administered mifepristone in vivo. Mol Genet Genomics, 2002. 268(2): p. 169-78.

87. Abruzzese, R.V., et al., Ligand-dependent regulation of plasmid-based transgene expression in vivo. Hum Gene Ther, 1999. 10(9): p. 1499-507.

88. Nordstrom, J.L., The antiprogestin-dependent GeneSwitch system for regulated gene therapy. Steroids, 2003. 68(10-13): p. 1085-94.

89. Burcin, M.M., et al., Adenovirus-mediated regulable target gene expression in vivo. Proc Natl Acad Sci U S A, 1999. 96(2): p. 355-60.

90. Oligino, T., et al., Drug inducible transgene expression in brain using a herpes simplex virus vector. Gene Ther, 1998. 5(4): p. 491-6.

91. Blandini, F. and M.T. Armentero, Animal models of Parkinson's disease. FEBS J, 2012. 279(7): p. 1156-66.

92. Munoz-Soriano, V. and N. Paricio, Drosophila models of Parkinson's disease: discovering relevant pathways and novel therapeutic strategies. Parkinsons Dis, 2011. 2011: p. 520640.

93. Harvey, B.K., Y. Wang, and B.J. Hoffer, Transgenic rodent models of Parkinson's disease. Acta Neurochir Suppl, 2008. 101: p. 89-92. 
94. Lee, Y., V.L. Dawson, and T.M. Dawson, Animal models of Parkinson's disease: vertebrate genetics. Cold Spring Harb Perspect Med, 2012. 2(10).

95. Taschenberger, G., et al., Aggregation of alphaSynuclein promotes progressive in vivo neurotoxicity in adult rat dopaminergic neurons. Acta Neuropathol, 2012. 123(5): p. 671-83.

96. Kirik, D., et al., Nigrostriatal alpha-synucleinopathy induced by viral vector-mediated overexpression of human alpha-synuclein: a new primate model of Parkinson's disease. Proc Natl Acad Sci U S A, 2003. 100(5): p. 2884-9.

97. Good, C.H., et al., Impaired nigrostriatal function precedes behavioral deficits in a genetic mitochondrial model of Parkinson's disease. FASEB J, 2011. 25(4): p. 1333-44.

98. Langston, J.W., et al., Chronic Parkinsonism in humans due to a product of meperidine-analog synthesis. Science, 1983. 219(4587): p. 979-80.

99. Hunter, R.L., et al., Intrastriatal lipopolysaccharide injection induces parkinsonism in C57/B6 mice. J Neurosci Res, 2009. 87(8): p. 1913-21.

100. Ungerstedt, U., T. Ljungberg, and G. Steg, Behavioral, physiological, and neurochemical changes after 6-hydroxydopamine-induced degeneration of the nigro-striatal dopamine neurons. Adv Neurol, 1974. 5: p. 421-6.

101. Lee, Y., et al., GFAP promoter elements required for region-specific and astrocyte-specific expression. Glia, 2008. 56(5): p. 481-93.

102. Grimm, D., et al., Novel tools for production and purification of recombinant adenoassociated virus vectors. Human Gene Therapy, 1998. 9(18): p. 2745-2760.

103. Loyter, A., G.A. Scangos, and F.H. Ruddle, Mechanisms of DNA uptake by mammalian cells: fate of exogenously added DNA monitored by the use of fluorescent dyes. Proc Natl Acad Sci U S A, 1982. 79(2): p. 422-6.

104. Zolotukhin, S., et al., Recombinant adeno-associated virus purification using novel methods improves infectious titer and yield. Gene Therapy, 1999. 6(6): p. 973-985.

105. de Hoop M.J., M.L., and otti C. G., Culturing hippocampal neurons and astrocytes from fetal rodent brain, in Academic Press. 1998.

106. Burnette, W.N., Western Blotting - Electrophoretic Transfer of Proteins from Sodium Dodecyl Sulfate-Polyacrylamide Gels to Unmodified Nitrocellulose and Radiographic Detection with Antibody and Radioiodinated Protein-A. Analytical Biochemistry, 1981. 112(2): p. 195-203.

107. Mostany, R. and C. Portera-Cailliau, A craniotomy surgery procedure for chronic brain imaging. J Vis Exp, 2008(12).

108. Schneider, C.A., W.S. Rasband, and K.W. Eliceiri, NIH Image to ImageJ: 25 years of image analysis. Nat Methods, 2012. 9(7): p. 671-5.

109. Fitzsimmons, D.F., T.C. Moloney, and E. Dowd, Further validation of the corridor task for assessing deficit and recovery in the hemi-Parkinsonian rat: Restoration of bilateral food retrieval by dopamine receptor agonism. Behavioural Brain Research, 2006. 169(2): p. 352355.

110. Sawamoto, K., et al., Visualization, direct isolation, and transplantation of midbrain dopaminergic neurons. Proc Natl Acad Sci U S A, 2001. 98(11): p. 6423-8.

111. Blits, B., et al., Adeno-associated viral vector (AAV)-mediated gene transfer in the red nucleus of the adult rat brain: comparative analysis of the transduction properties of seven AAV serotypes and lentiviral vectors. J Neurosci Methods, 2010. 185(2): p. 257-63.

112. Flotte, T.R., et al., Expression of the cystic fibrosis transmembrane conductance regulator from a novel adeno-associated virus promoter. J Biol Chem, 1993. 268(5): p. 3781-90.

113. Seiler, M.P., et al., Adeno-associated virus types 5 and 6 use distinct receptors for cell entry. Hum Gene Ther, 2006. 17(1): p. 10-9.

114. Shevtsova, Z., et al., Potentiation of in vivo neuroprotection by $B C I X(L)$ and GDNF coexpression depends on post-lesion time in deafferentiated CNS neurons. Gene Ther, 2006. 13(22): p. 1569-78. 
115. Fan, D.S., et al., Behavioral recovery in 6-hydroxydopamine-lesioned rats by cotransduction of striatum with tyrosine hydroxylase and aromatic L-amino acid decarboxylase genes using two separate adeno-associated virus vectors. Hum Gene Ther, 1998. 9(17): p. 2527-35.

116. Millington-Ward, S., et al., Suppression and replacement gene therapy for autosomal dominant disease in a murine model of dominant retinitis pigmentosa. Mol Ther, 2011. 19(4): p. 642-9.

117. Palfi, A., et al., Efficacy of codelivery of dual AAV2/5 vectors in the murine retina and hippocampus. Hum Gene Ther, 2012. 23(8): p. 847-58.

118. Christine, C.W., et al., Safety and tolerability of putaminal AADC gene therapy for Parkinson disease. Neurology, 2009. 73(20): p. 1662-1669.

119. Kaplitt, M.G., et al., Safety and tolerability of gene therapy with an adeno-associated virus (AAV) borne GAD gene for Parkinson's disease: an open label, phase I trial. Lancet, 2007. 369(9579): p. 2097-2105.

120. Kordower, J.H. and C.W. Olanow, Regulatable promoters and gene therapy for Parkinson's disease: is the only thing to fear, fear itself? Exp Neurol, 2008. 209(1): p. 34-40.

121. Cress, D.E., The need for regulatable vectors for gene therapy for Parkinson's disease. Experimental Neurology, 2008. 209(1): p. 30-33.

122. Ginhoux, F., et al., HLA-A*0201-restricted cytolytic responses to the rtTA transactivator dominant and cryptic epitopes compromise transgene expression induced by the tetracycline on system. Molecular Therapy, 2004. 10(2): p. 279-289.

123. Baulieu, E.E., et al., Are receptor-associated nuclear proteins associated with the earliest effects of steroid hormones? Rev Esp Fisiol, 1990. 46(1): p. 17-29.

124. Gallagher, P. and A.H. Young, Mifepristone (RU-486) treatment for depression and psychosis: a review of the therapeutic implications. Neuropsychiatr Dis Treat, 2006. 2(1): p. 33-42.

125. Szymanski, P., et al., Development and validation of a robust and versatile one-plasmid regulated gene expression system. Mol Ther, 2007. 15(7): p. 1340-7.

126. Drinkut, A., et al., Efficient Gene Therapy for Parkinson's Disease Using Astrocytes as Hosts for Localized Neurotrophic Factor Delivery. Molecular Therapy, 2012. 20(3): p. 534-543.

127. Zeng, X., et al., An in vitro model of human dopaminergic neurons derived from embryonic stem cells: MPP+ toxicity and GDNF neuroprotection. Neuropsychopharmacology, 2006. 31(12): p. 2708-15.

128. Scholz, D., et al., Rapid, complete and large-scale generation of post-mitotic neurons from the human LUHMES cell line. J Neurochem, 2011. 119(5): p. 957-71.

129. Pu, J.L., et al., Redefining Parkinson's Disease Research Using Induced Pluripotent Stem Cells. Current Neurology and Neuroscience Reports, 2012. 12(4): p. 392-398.

130. Lee, W.Y., et al., Vesicular monoamine transporter-2 and aromatic L-amino acid decarboxylase enhance dopamine delivery after L-3, 4-dihydroxyphenylalanine administration in Parkinsonian rats. J Neurosci, 1999. 19(8): p. 3266-74.

131. Asokan, A., D.V. Schaffer, and R.J. Samulski, The AAV vector toolkit: poised at the clinical crossroads. Mol Ther, 2012. 20(4): p. 699-708. 


\section{Annexes}

\subsection{Abbreviations}

\section{6- OHDA = 6-hydroxy dopamine}

AADC $=$ Aromatic L-amino acid decarboxylase

AAV = Adeno Associated Virus

$\mathbf{A R}=$ antigen retrieval

ARE $=$ antioxidant responsive element

$\alpha$-syn $=\alpha$ synuclein

$\mathbf{B}=\mathbf{B G H p A}=$ bovine growth hormone poli adenilation sequence

BB $=$ back bone (linearized vector genome)

BBB $=$ Blood brain barrier

BCA = bicinchoninic acid

BH4 = Tetrahydrobiopterin

$\mathbf{B p}=$ base pairs

BSA = bovine serum albumine

$\mathbf{C F}=$ cell factory

CMV = cytomegalovirus

CNS = Central nervous system

DA = dopamine/dopamiregic

DAT $=$ Dopamine transporter

DBD $=$ DNA binding domain

DIV = day in vitro

DMEM = Dulbecco's modified Eagle's medium

DOPAC $=3,4-$ Dihydroxyphenylacetic acid

Dox $=$ doxycycline

EDTA = ethylenediaminetetraacetic acid

EGFP = Enhanced green fluorescence protein

FCS $=$ fetal calf serum

FDA $=$ Food and drug administration

FPLC $=$ Fast protein liquid chromatography

GAPDH = Glyceraldehyde 3-phosphate dehydrogenase

GCH1 = GTP cyclohydrolase 1

GDNF = Glial cell derived neurotrophic factor

$\mathbf{G E}=$ DNA gel extraction 
GFAP = Glial fibrillary acidic protein

GOI = gene of interest

$\mathbf{L} \mathbf{V}=$ Lentivirus

HCN = hippocampus and cortex primary culture medium

HPLC = High pressure liquid chromatography

hPR-LBD = human progesterone recptor ligand binding domain

$\mathbf{H R P}=$ horse reddish peroxidise

HSV $=$ Heprex Simplex virus

HVA = homovanillic acid

IHC = Immunohistochemistry

HRE = Hypoxia responsive element

ITR $=$ inverted terminal repeats

Kan $=$ kanamicin

LB = Lysogeny broth

MF = Mifepristone = 11 $\beta$-[4-dimethylamino]phenyl-17 $\beta$-hydroxy-17-[1-propynyl]estra-4,9-dien-3-

one)

MPTP = 1-methyl-4-phenyl-1,2,3,6-tetrahydropyridine

NDD = neurodegenerative disease

$\mathbf{N e u N}=$ neuron specific nuclear protein

$\mathbf{N F}=$ neurotrophic factor

NGS = newborn goat serum

NTN = neurturin

ORF = open reading frame

pA = poli adenilation sequence

PBS = phosphate buffered saline

$\mathbf{P C R}=$ polymerase chain reaction

PD = Parkinson's disease

PFA = paraformaldehyde

$\mathbf{P P}=\mathbf{P C R}$ purification

PS = penicillin/streptomycin

PS-N = penicillin/streptomycin/Neomicin

$\mathbf{p S w}=p S w i t c h$ protein

ptk $=$ Herpes Simplex virus thymidine kinase $(\mathrm{tk})$ minimal promoter

$\mathbf{R B}=$ Releasing buffer

Rap $=$ rapamycin 
$\mathbf{R O I}=$ region of interest

ROS $=$ Reactive oxygen species

rpm $=$ rotations per minute

$\mathbf{R T}=$ room temperature

$\mathbf{S}$ (before the expressed protein in the construct $=\mathbf{s y n}=$ sinapsin promoter

$\mathbf{S}$ (after the expressed protein in the construct $)=\mathbf{S V 4 0}=$ Simian virus 40 poli adenilation sequence

SD = standard deviation

Sn $=$ Substantia nigra

Snpc = Substantia nigra pars compacta

Tc $=$ tetracycline

TH = tyrosine hydroxylase

UAS = Upstream activatin sequence

Uptk = UAS Herpes Simplex Virus thymidine kinase (TK) minimal promoter

UTR = untranslatede region

Vmat-2 = Vesicular monoamine transporter 2

WPRE $=$ Woodchuck Hepatitis Virus (WHP) Posttranscriptional Regulatory Element 


\title{
8.2 Curriculum vitae
}

\author{
Andrea Maddalena \\ Von Ossietzky strasse 4 \\ 37085 Göttingen \\ Germany \\ Mobile phone: +49 17681139595 \\ E-mail address: andreamadda@hotmail.it
}

\section{Personal Information}

Date of birth:

26 March, 1983

Place of birth:

Schio (Vicenza), Italy

Marital status:

Single

Nationality:

Italian

\section{Research Experience}

\section{University of Göttingen}

Göttingen, Germany

December 2008 - Now

Universitätmedizin Göttingen

Department of neurology

Faculty of biology

Phd thesis:

In progress

\section{University of Geneva}

Geneva, Switzerland

March 2007 - March 2008

Faculty of Medicine

Department of Pathology and Immunology

Graduate thesis in Biotechnology:

"Use of lentiviral vectors and cardiogenesis in human embryonic stem cells".

\section{University of Padova}

Padova, Italy

May 2005 - July 2005

Faculty of Biotechnology

Venetian Institute of Molecular Medecine (VIMM)

Undergraduate thesis in Biotechnology:

"Characterization of channel's activity of Vibrio Cholerae Citolysin (Vcc)" 


\section{Education}

December 2008 - Now University of Göttingen, Germany

Phd thesis

October 2005 - March 2008 Università degli Studi di Padova, Italy. Laurea Specialistica (Master) in Biotecnologie Industriali.

Curriculum: Proteine ricombinanti e immunologia.

Degree Thesis: "Use of lentiviral vectors and cardiogenesis in human embryonic stem cells". Under supervision of Dr. Marisa Jaconi; Centre Medical Universitaire (CMU) de Geneve.

Final mark: 106/110

September 2002 - July 2005 Università degli Studi di Padova, Italy Laurea (Bachelor)in biotecnologie industriali.

Degree Thesis:"Characterization of channel's activity of

Vibrio Cholerae Citolysin (Vcc)" under supervision of Marina De Bernard at VIMM (Venetian Institute of Molecular Medicine).

Final mark: 98/110

September 1997 - July 2002 Istituto Superiore: Liceo scientifico N.Tron Schio VI

Diploma di Maturità Scientifica

Final mark: $70 / 100$

\section{Additional Skills}

\section{Activities connected to the Ph.D.:}

CMPB student representative: march 2011-march 2012

Symposia: Organizer of $1^{\text {st }}$ international CMPB symposium

Posters: 6-7 posters: main title $\rightarrow$ In vivo imaging of regulated transgene expression in mice and rat brain

\section{Computer skills:}

Microsoft Office (Word, Excel, Power Point), EndNote, Zeiss Imageviewer, Image J, various software for microscope, photoshop, various....

\section{Languages:}

Italian: Native language

English: Adept at speaking and writing

French: Good in speaking

German: Basic 


\subsection{Publications}

HDAC1 regulates fear extinction in mice.

Bahari-Javan S, Maddalena A, Kerimoglu C, Wittnam J, Held T, Bähr M, Burkhardt S, Delalle I, Kügler $S$, Fischer A, Sananbenesi F.

Department of Psychiatry and Psychotherapy, University Medical Center Göttingen, 37075 Göttingen, Germany.

J Neurosci. 2012 Apr 11;32(15):5062-73.

Adeno-associated virus mediated, mifepristone-regulated neurotrophic factor expression in the brain

Andrea Maddalena, Julia Tereshchenko, Mathias Bähr, Sebastian Kügler

University Medicine Goettingen, Department of Neurology

Article submitted 


\section{Acknowledgment}

Here we are...Finally...

Wow...Soooo many people to thanks...Unluckily I have to be quick....

Per prima cosa ringrazio la mia famiglia che mi ha sempre sostenuto e incoraggiato nel seguire le mie aspirazioni. $\mathrm{E}$ a parte questo $\mathrm{mi}$ ha sempre fatto trovare un letto con lenzuola fresche quando tornavo a casa nonché decine dei miei piatti preferiti. Quindi un grazie enorme vá a Mamma, Papá, e Barbara. Ovviamente un abbraccio enorme a nonna e zia che mi hanno sempre coccolato.

Coming back to Göttingen I first have to thank the whole group for the awesome support I got in these 4 years. Thanks to Sebastian for the great supervision and for being patient with my timeschedule. My experience in this lab was so positive especially because the group was leaded in a great way. I then thank Yuliya with whom I shared this project...Our discussions have been countless but they always lead to good results. It has been nice and productive to work with you; I've learnt a lot on how to be more ordered. I thank a lot Ulrike, Monica and Grit for the material and moral support in the last years. I don't know if I would have finished without your support. Of course thanks to Johan!! A part of helping at work I've always had good and relaxing time in mensa and in the occasions we had spent time together!!! I thank as well all the rest of the people in Waldweg 33 for the support in these years...It will be too long to name everybody but THANKS!!!

Tornando in Italia ringrazio tutti I miei amici di sempre!! Ogni volta che tornavo mi facevate sentire come se non fossi mai partito!!!! Uuhh...Nominarvi tutti é un bel casino....Vabbé...Un saluto particolare a Giane, Branda, Maxi, Fede, Carlo...Non facevo tempo a tornare che giá mi chiamavate... Passando all'altro sesso ringrazio Francesca per essere stata la mia psicologa (mi hai aiutato)...Un saluto particolare anche a Silvia, Cia, Vale, Paola per gli innumerevoli aperitivi a cui riuscivate sempre a venire!! Un congratulazioni a Tonzi e Chiara che stanno per diventare genitori!!!E'stato divertente farvi da testimone!!!! Sicuramente ho dimenticato qualcuno in questa veloce lista...Non prendetevela...In questo momento sono cotto...Ma mando un abbraccio a tutti!!!!

Ok...Let's go back to Göttingen again ...Another difficult job to remember everybody I would like to thank and I'm quite tired (read "fucking tired")...So...These 4 years have been unforgettable especially for the great people I met...It's cool..People from everywhere getting along and enjoying...Everybody with their own characteristics...This was really enriching!!! Starting from the beginning I met Chriss...Shit...Too many parties...Awesome...Going on more or less in a time way I thank lliana for taking care of me countless times...Going on a bit more I thank Matthieu (le connard français) for party and listening, Liam as well, Jonas, Alwaleed, Ahmed (weird guy...but cool... :) ), Andrew (your turkeys are awesome!!), Stephanie (my former psychologist), Derya, Natalia, Samir, Anne, Marie for the countless stuff we have done together in these years!!! And a hug to Sanaz with whom I've been writing in these last months!! It was great to meet some other Italians as well to joke about the rests $(-)$ : sempre divertente passare serate con voi Gio e lla (secchiona)!!!!! Göttingen is nice because it brings everytime new people...Nice parties and discussion with Tania, Tina, Andonia, Sybille ecc....Un saluto speciale a Sara con la quale ho iniziato questa avventura..Sono felice che siamo restati amici...And thanks again to everybody for having supported me in this last period..I was really impressed and happy!!!!!!!!!

I thank Paul and my football team Puma Göttingen...Football and beer...The best for relaxing!!! 
Finally now I'm in a nice WG and for that I thank Artyom and Sven!!!

Some random thanks go to Sonder bar and Brancamenta, to caffeine, to the various kebabs in the city, to all the clubs for partying and forget all the problems in the lab.

I have to go now...If I forgot somebody I'm sorry...Anyway I hope to thank each of you personally!!!!

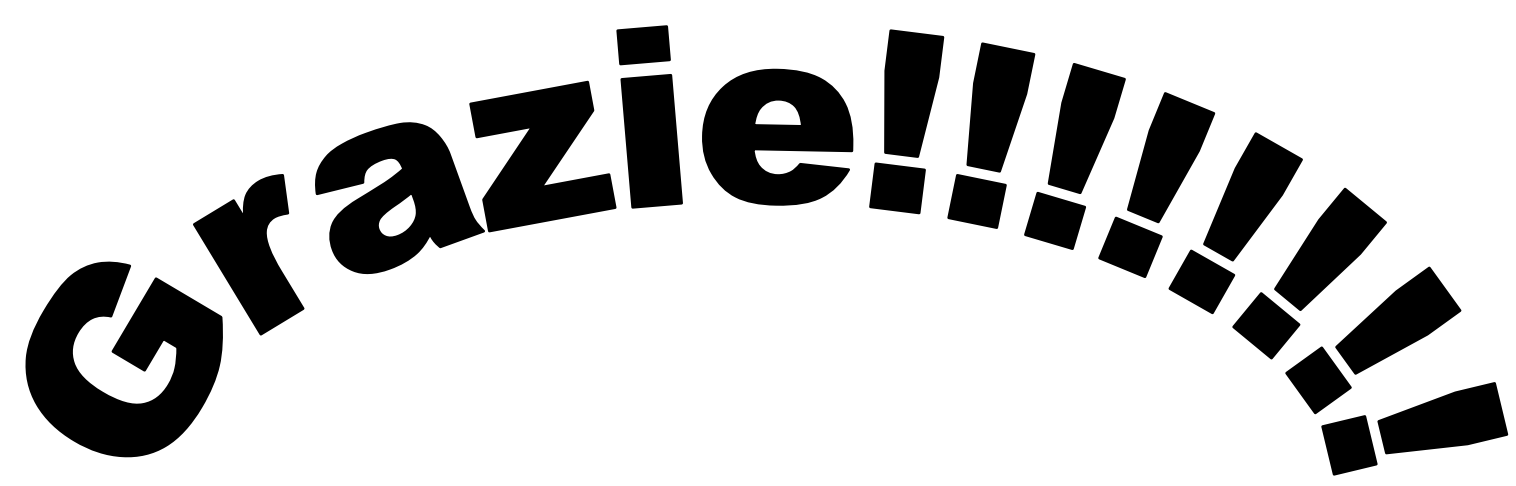

